\title{
Palladium-Catalyzed C-H Silylation of Aliphatic Ketones Using an Aminooxyamide Auxiliary
}

\author{
Jianhua Li, Chao Jiang* \\ School of Chemistry and Chemical Engineering, Nanjing University of Science and Technology, \\ Nanjing, Jiangsu 210094 \\ Email: chaojiang@njust.edu.cn, yu200@scripps.edu
}

\section{Table of Contents}

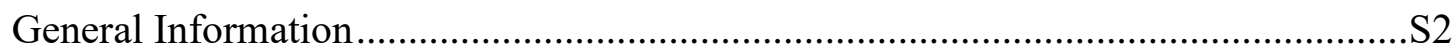

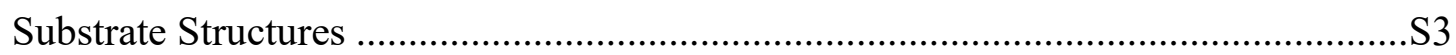

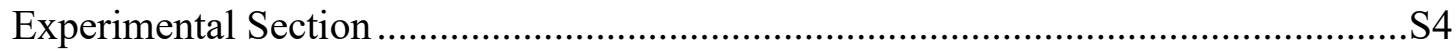

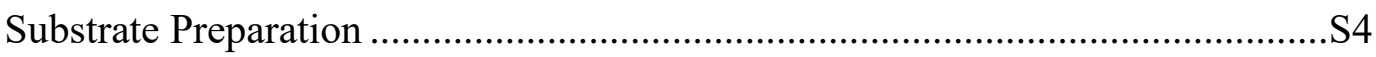

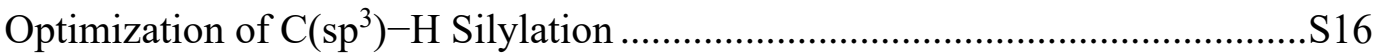

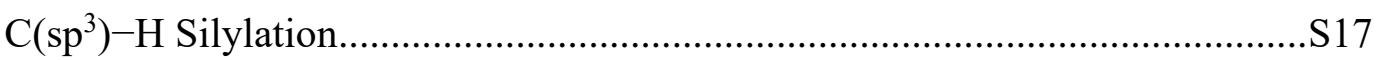

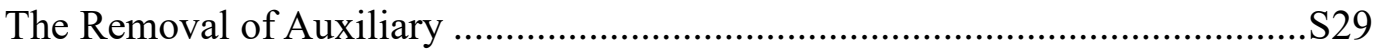

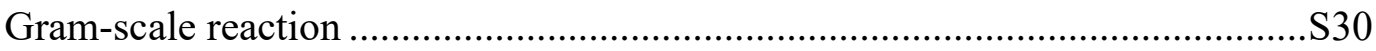

The Late-Stage $\beta-\mathrm{C}\left(\mathrm{sp}^{3}\right)-\mathrm{H}$ Silylation of Santonin .......................................S30

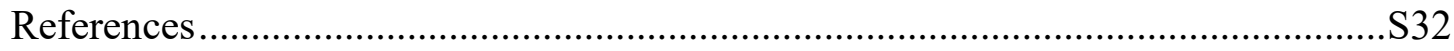

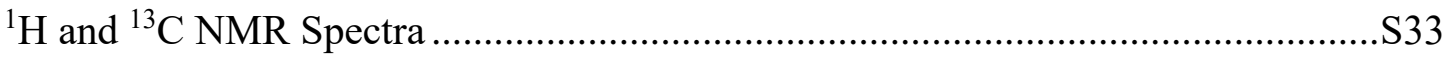




\section{General Information}

Ketones were obtained from the commercial sources or synthesized following literature procedures, and used to prepare the corresponding substrates. Aminooxyacetic acid hemihydrochloride was obtained from Meryer, octafluorotluene was obtained from Fluorochem, $\mathrm{Pd}(\mathrm{OAc})_{2}$ was obtained from Heowns, Hexamethyldisilane was from Meryer, AgTFA was from Meryer, $\mathrm{Li}_{2} \mathrm{CO}_{3}$ was from Titan and $\mathrm{Cu}(\mathrm{OAc})_{2} \cdot \mathrm{H}_{2} \mathrm{O}$ was from Energy Chemical. Solvents were obtained from Nanjing Chemical Reagent and used directly without further purification. Analytical thin layer chromatography was performed on $0.5 \mathrm{~mm}$ silica gel. Visualization was carried out with UV light. ${ }^{1} \mathrm{H}$ NMR was recorded on Bruker AV-500 instrument (500 MHz). Chemical shifts were quoted in parts per million (ppm) referenced to $0.0 \mathrm{ppm}$ for tetramethylsilane. The following abbreviations (or combinations thereof) were used to explain multiplicities: $\mathrm{s}=$ singlet, $\mathrm{d}=$ doublet, $\mathrm{t}=$ triplet, $\mathrm{q}=$ quartet, $\mathrm{m}=$ multiplet, $\mathrm{br}=$ broad. Coupling constants, $J$, were reported in Hertz unit (Hz). ${ }^{13} \mathrm{C}$ NMR spectra were recorded on Bruker AV-500 instrument (126 MHz), and were fully decoupled by broad band proton decoupling. Chemical shifts were reported in ppm referenced to either the center line of a triplet at $77.0 \mathrm{ppm}$ of chloroform- $d$. In the ${ }^{13} \mathrm{C}$ NMR analysis, peaks that correspond to those of the polyfluoroarylamide auxiliary appeared as nearly invisible, complex sets of multiplets; they were omitted in the following spectroscopic analysis. High Resolution Mass spectra were obtained from on an Agilent Mass spectrometer using ESI-TOF (electrospray ionization-time of flight) or Waters GCT Premeier Mass Spectrometer. 


\section{Substrate Structures}
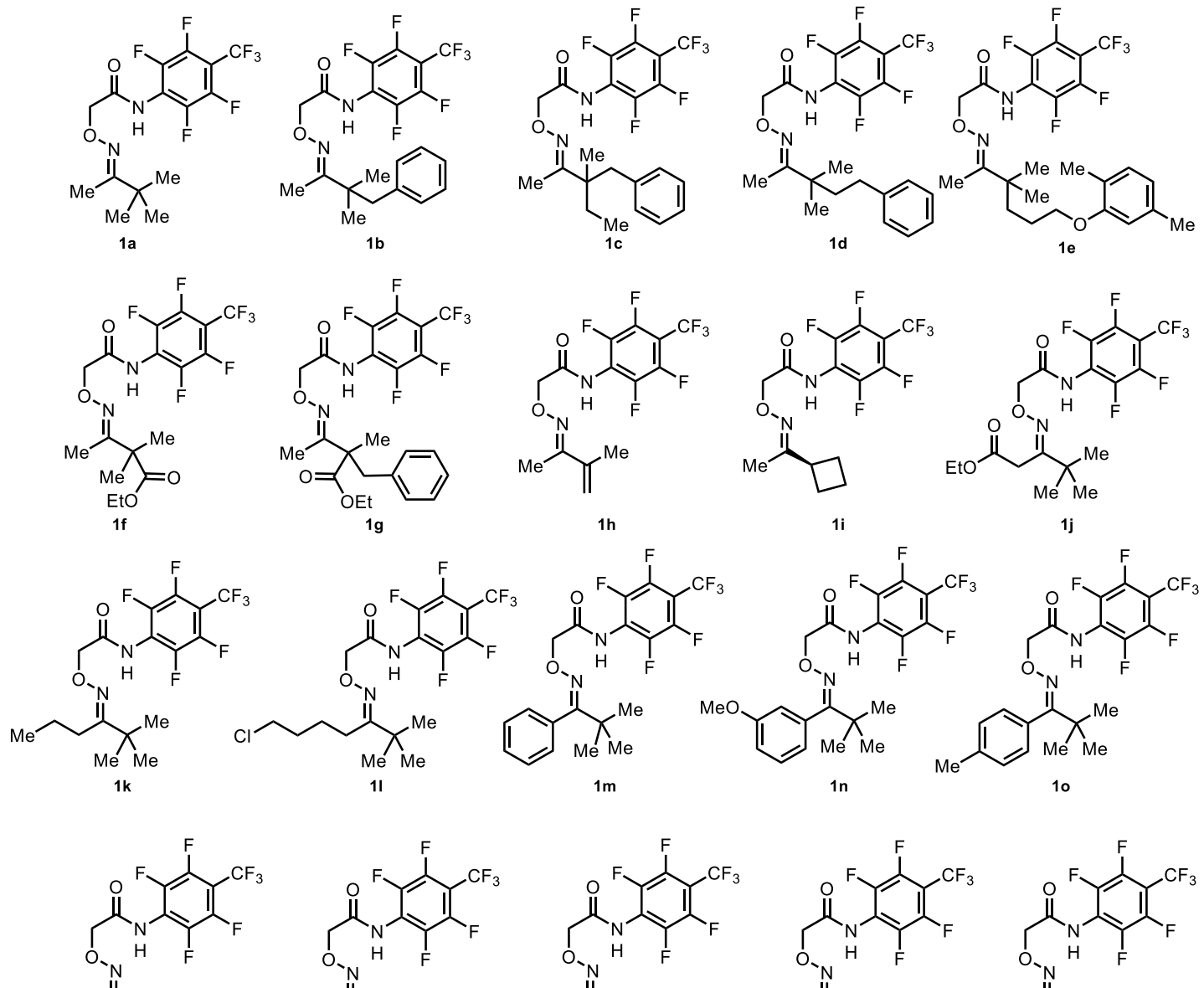

每

19

1r 1s

1t

(N) 12<smiles>CC1=C2[C@@H]3OC(=O)[C@H](C)[C@@H]3CC[C@]2(C)C=C/C1=N\OCC(=O)Nc1c(F)c(F)c(C(F)(F)F)c(F)c1F</smiles> 


\section{Experimental Section}

\section{Substrate Preparation}

Ketones for substrates $\mathbf{1 a}, \mathbf{1 h}, \mathbf{1} \mathbf{i}, \mathbf{1} \mathbf{j}, \mathbf{1} \mathbf{m}$ and $\mathbf{1 z}$ are commercial available. Ketones for substrates $\mathbf{1 b}, \mathbf{1 c}$ and $\mathbf{1 d}$ were synthesized following literature procedures. ${ }^{1}$ Ketones for substrates 1e, 1f and 1g were synthesized by the reaction between corresponding carboxylic acids and methyllithium. The procedure can be found in literature. ${ }^{2}$ Ketones for substrate 1n, 1o, 1p, 1q, 1r, 1 s, 1 t were synthesized following literature procedures. ${ }^{3}$ Ketones for substrate $\mathbf{1 k}$ and $\mathbf{1 l}$ were synthesized following literature procedures. ${ }^{4}$ Ketones for substrate $\mathbf{1 u}, \mathbf{1 v}, \mathbf{1 w}, \mathbf{1 x}, \mathbf{1 y}$ were synthesized following literature procedures. $^{5}$

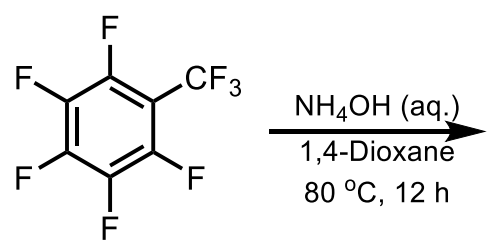<smiles>Nc1c(F)c(F)c(C(F)(F)F)c(F)c1F</smiles>

Preparation of 2,3,5,6-Tetrafluoro-4-(trifluoromethyl)aniline: ${ }^{6}$ To a solution of octafluorotoluene $(30 \mathrm{~mL})$ in 1,4-dioxane $(79 \mathrm{~mL})$ was added aqueous $\mathrm{NH}_{4} \mathrm{OH}(53 \mathrm{~mL}$, $28 \% \mathrm{w} / \mathrm{w}$ ), and the reaction flask was sealed and heated to $80{ }^{\circ} \mathrm{C}$ in an oil bath. The reaction mixture was stirred for 12 hours. The mixture was then cooled, and 1,4-dioxane was removed in vacuo. The resultant mixture was extracted with ethyl acetate $(3 \times 100$ $\mathrm{mL}$ ), and then concentrated in vacuo. The crude mixture was purified by column chromatography (Hexane : EtOAc $=4: 1$ ) to afford 2,3,5,6-tetrafluoro-4(trifluoromethyl)aniline as a colorless liquid (29.6 g, 60\%).<smiles>[R]C(=O)C([R])([R])CC</smiles><smiles>C1CCCCC1</smiles>
$\underset{60{ }^{\circ} \mathrm{C}, 2 \mathrm{~h}}{\stackrel{\text { Pyridine }}{(0.4 \mathrm{M})}}$<smiles>[R]/C(=N/OCC(=O)Nc1c(F)c(F)c(C(F)(F)F)c(F)c1F)C([R])([R])CC</smiles>

$$
\begin{aligned}
& \text { 1) }(\mathrm{COCl})_{2} \text { (1.2 equiv) } \\
& \text { DCM }(0.05 \mathrm{M}) \\
& \mathrm{N}_{2}, \mathrm{rt}, 0.5 \mathrm{~h} \\
& \text { 2) } \mathrm{ArFNH}_{2} \text { (1.1 equiv) } \\
& \text { Toluene }(0.1 \mathrm{M}) \\
& \text { Reflux, } 12 \mathrm{~h}
\end{aligned}
$$
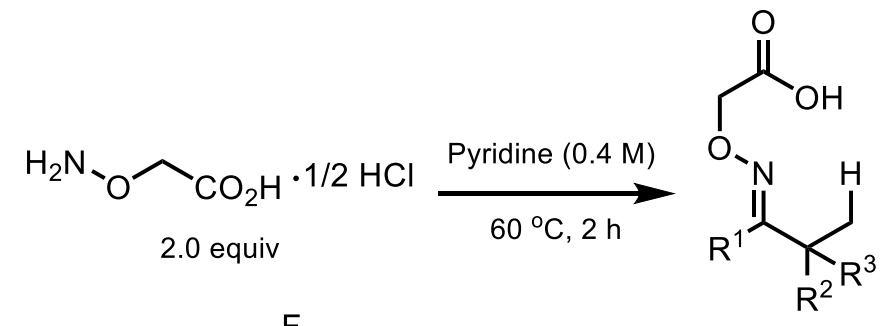

General Procedure for the Preparation of Substrates: Step1: Ketone (2.0 mmol, 1.0 equiv) and aminooxyacetic acid hemihydrochloride ( $4.0 \mathrm{mmol}, 437 \mathrm{mg}, 2.0$ equiv) were 
weighed into an oven dried $50 \mathrm{~mL}$ round bottom flask with a magnetic stir bar under air. $5 \mathrm{~mL}$ Pyridine was added and the mixture was stirred at $60^{\circ} \mathrm{C}$ in an oil bath for $2 \mathrm{~h}$. Upon completion, most pyridine was evaporated under vacuum. The resulting mixture was diluted with EtOAc $(50 \mathrm{~mL})$ and washed successively with water $(100 \mathrm{~mL})$ and diluted $\mathrm{HCl}$ aqueous solution $(100 \mathrm{~mL}$, ca. $0.01 \mathrm{M})$. The organic phase was dried with anhydrous $\mathrm{Na}_{2} \mathrm{SO}_{4}$ and the solvent was removed under vacuum. Notably, the pure compounds were obtained in good yields for all cases without chromatography.

Step 2: An acid chloride $(1.0 \mathrm{mmol})$, prepared from the corresponding carboxylic acid (1.0 mmol) and oxalyl chloride (1.2 mmol, $152.4 \mathrm{mg}, 1.2$ equiv), was added to a vigorously stirred solution of 2,3,5,6-tetrafluoro-4-(trifluoromethyl)aniline (1.1 mmol) in toluene $(10 \mathrm{~mL})$. The reaction mixture was stirred for 12 hours under reflux in an oil bath. The resultant mixture was extracted with ethyl acetate $(3 \times 100 \mathrm{~mL})$, and then concentrated in vacuo. The crude mixture was purified by column chromatography (Hexane : $\mathrm{EtOAc}=9: 1)$ to afford corresponding substrates.<smiles>C/C(=N\OCC(=O)Nc1c(F)c(F)c(C(F)(F)F)c(F)c1F)C(C)(C)C</smiles>

(E)-2-(((3,3-dimethylbutan-2-ylidene)amino)oxy)-N-(2,3,5,6-tetrafluoro-4-(trifluo romethyl)phenyl)acetamide (1a)

White solid (519.9 mg, 67\%). ${ }^{1} \mathrm{H}$ NMR (500 MHz, $\left.\mathrm{CDCl}_{3}\right) \delta 7.69$ (br s, $\left.1 \mathrm{H}\right), 4.59$ (s, 2H), 1.90 (s, 3H), 1.12 (s, 9H). ${ }^{13} \mathrm{C} \mathrm{NMR} \mathrm{(126} \mathrm{MHz,} \mathrm{CDCl3)} \delta 168.8,168.6,72.3,37.6$, 27.4, 11.0. HRMS (ESI-TOF) m/z: [M-H] $]^{-}$calculated for $\mathrm{C}_{15} \mathrm{H}_{14} \mathrm{~F}_{7} \mathrm{~N}_{2} \mathrm{O}_{2}{ }^{-} 387.0944$, found 387.0954 .<smiles>C/C(=N/OCC(=O)Nc1c(F)c(F)c(C(F)(F)F)c(F)c1F)C(C)(C)Cc1ccccc1</smiles>

(E)-2-(((3,3-dimethyl-4-phenylbutan-2-ylidene)amino)oxy)-N-(2,3,5,6-tetrafluoro4-(trifluoromethyl)phenyl)acetamide (1b)

White solid (555.6 mg, 60\%). ${ }^{1} \mathrm{H}$ NMR (500 MHz, $\left.\mathrm{CDCl}_{3}\right) \delta 7.69$ (br s, $\left.1 \mathrm{H}\right), 7.10$ (d, $J$ $=7.6 \mathrm{~Hz}, 2 \mathrm{H}), 7.02(\mathrm{~d}, J=7.2 \mathrm{~Hz}, 2 \mathrm{H}), 6.86(\mathrm{t}, J=7.4 \mathrm{~Hz}, 1 \mathrm{H}), 4.60(\mathrm{~s}, 2 \mathrm{H}), 2.76(\mathrm{~s}$, $2 \mathrm{H}), 2.05(\mathrm{~s}, 3 \mathrm{H}), 1.16(\mathrm{~s}, 6 \mathrm{H}) .{ }^{13} \mathrm{C} \mathrm{NMR}\left(126 \mathrm{MHz}, \mathrm{CDCl}_{3}\right) \delta 169.0,166.8,137.9$, 130.1, 127.7, 125.9, 72.1, 46.3, 42.0, 25.2, 12.0. HRMS (ESI-TOF) m/z: [M-H] $]^{-}$ 
calculated for $\mathrm{C}_{21} \mathrm{H}_{18} \mathrm{~F}_{7} \mathrm{~N}_{2} \mathrm{O}_{2}{ }^{-} 463.1257$, found 463.1270 .<smiles>CCC(C)(Cc1ccccc1)C(C)=NOCC(=O)Nc1c(F)c(F)c(C(F)(F)F)c(F)c1F</smiles>

(E)-2-(((3-benzyl-3-methylpentan-2-ylidene)amino)oxy)-N-(2,3,5,6-tetrafluoro-4(trifluoromethyl)phenyl)acetamide (1c)

White solid (621.4 mg, 65\%). ${ }^{1} \mathrm{H}$ NMR (500 MHz, $\left.\mathrm{CDCl}_{3}\right) \delta 7.67$ (br s, 1H), 7.09 (d, J $=7.6 \mathrm{~Hz}, 2 \mathrm{H}), 7.01(\mathrm{~d}, J=7.1 \mathrm{~Hz}, 2 \mathrm{H}), 6.84(\mathrm{t}, J=7.4 \mathrm{~Hz}, 1 \mathrm{H}), 4.61(\mathrm{~d}, J=5.2 \mathrm{~Hz}$, 2H), $2.84(\mathrm{~s}, 1 \mathrm{H}), 2.65(\mathrm{~d}, J=13.6 \mathrm{~Hz}, 1 \mathrm{H}), 2.03(\mathrm{~s}, 3 \mathrm{H}), 1.76(\mathrm{dq}, J=14.7,7.5 \mathrm{~Hz}$, 1H), 1.49-1.41 (m, 1H), $1.05(\mathrm{~s}, 3 \mathrm{H}), 0.84(\mathrm{t}, J=7.5 \mathrm{~Hz}, 3 \mathrm{H}) .{ }^{13} \mathrm{C}$ NMR $(126 \mathrm{MHz}$, $\left.\mathrm{CDCl}_{3}\right) \delta 169.1,165.8,137.8,130.2,127.7,125.9,72.1,45.7,45.5,31.5,20.3,11.9$, 8.7. HRMS (ESI-TOF) m/z: [M-H] $]^{-}$calculated for $\mathrm{C}_{22} \mathrm{H}_{20} \mathrm{~F}_{7} \mathrm{~N}_{2} \mathrm{O}_{2}{ }^{-}$477.1413, found 477.1427 .<smiles>C/C(=N\OCC(=O)Nc1c(F)c(F)c(C(F)(F)F)c(F)c1F)C(C)(C)C</smiles>

(E)-2-(((3,3-dimethyl-5-phenylpentan-2-ylidene)amino)oxy)-N-(2,3,5,6-tetrafluor o-4-(trifluoromethyl)phenyl)acetamide (1d)

White solid (525.8 mg, 55\%). ${ }^{1} \mathrm{H}$ NMR $\left(500 \mathrm{MHz}, \mathrm{CDCl}_{3}\right) \delta 8.08$ (br s, $\left.1 \mathrm{H}\right), 7.27(\mathrm{t}, J$ $=7.6 \mathrm{~Hz}, 2 \mathrm{H}), 7.19(\mathrm{~d}, J=7.3 \mathrm{~Hz}, 1 \mathrm{H}), 7.13(\mathrm{~d}, J=7.4 \mathrm{~Hz}, 2 \mathrm{H}), 4.72(\mathrm{~s}, 2 \mathrm{H}), 3.11(\mathrm{~d}$, $J=4.8 \mathrm{~Hz}, 1 \mathrm{H}), 2.55-2.42(\mathrm{~m}, 2 \mathrm{H}), 1.95$ (s, 3H), 1.82-1.75 (m, 2H), 1.56 (s, 3H), 1.20 $(\mathrm{d}, J=4.4 \mathrm{~Hz}, 6 \mathrm{H}) .{ }^{13} \mathrm{C} \mathrm{NMR}\left(126 \mathrm{MHz}, \mathrm{CDCl}_{3}\right) \delta 168.7,167.3,142.1,128.5,128.2$, 126.0, 72.4, 42.3, 40.9, 31.2, 25.5, 11.1. HRMS (ESI-TOF) m/z: [M-H] ${ }^{-}$calculated for $\mathrm{C}_{22} \mathrm{H}_{20} \mathrm{~F}_{7} \mathrm{~N}_{2} \mathrm{O}_{2}{ }^{-} 477.1413$, found 477.1424. 
<smiles>CC(=NOCC(=O)Nc1c(F)c(F)c(C(F)(F)F)c(F)c1F)C(C)(C)CCCOc1cc(C)ccc1C</smiles>

(E)-2-(((6-(2,5-dimethylphenoxy)-3,3-dimethylhexan-2-ylidene)amino)oxy)-N-(2, 3,5,6-tetrafluoro-4-(trifluoromethyl)phenyl)acetamide (1e)

White solid (632.5 mg, 59\%). ${ }^{1} \mathrm{H}$ NMR (500 MHz, $\left.\mathrm{CDCl}_{3}\right) \delta 8.08$ (br s, $\left.1 \mathrm{H}\right), 7.00$ (t, $J$ $=7.5 \mathrm{~Hz}, 1 \mathrm{H}), 6.66(\mathrm{~d}, J=7.4 \mathrm{~Hz}, 1 \mathrm{H}), 6.59(\mathrm{~s}, 1 \mathrm{H}), 4.71(\mathrm{~s}, 2 \mathrm{H}), 3.92(\mathrm{~d}, J=15.0 \mathrm{~Hz}$, 2H), $2.31(\mathrm{~d}, J=4.5 \mathrm{~Hz}, 3 \mathrm{H}), 2.16(\mathrm{~d}, J=8.0 \mathrm{~Hz}, 3 \mathrm{H}), 1.99-1.92(\mathrm{~m}, 3 \mathrm{H}), 1.68(\mathrm{~s}, 4 \mathrm{H})$, 1.19 (s, 6H). ${ }^{13} \mathrm{C}$ NMR $\left(126 \mathrm{MHz}, \mathrm{CDCl}_{3}\right) \delta 168.7,167.4,156.8,136.6,130.4,123.4$, 120.8, 111.8, 67.7, 40.6, 36.5, 25.5, 24.9, 21.4, 15.7, 11.0. HRMS (ESI-TOF) m/z: [M$\mathrm{H}]^{-}$calculated for $\mathrm{C}_{25} \mathrm{H}_{26} \mathrm{~F}_{7} \mathrm{~N}_{2} \mathrm{O}_{3}{ }^{-}$535.1832, found 535.1845.<smiles>CCOC(=O)C(C)(C)C(C)=NOCC(=O)Nc1c(F)c(F)c(C(F)(F)F)c(F)c1F</smiles>

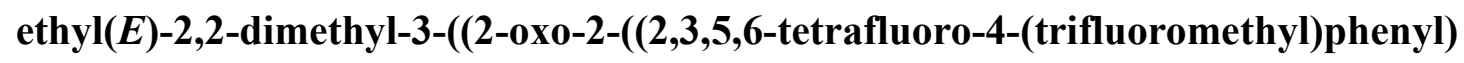
amino)ethoxy)imino)butanoate (1f)

White solid (562.0 mg, 63\%). ${ }^{1} \mathrm{H}$ NMR (500 MHz, $\left.\mathrm{CDCl}_{3}\right) \delta 8.01$ (br s, 1H), 4.76 (s, $2 \mathrm{H}), 4.19$ (d, $J=7.1 \mathrm{~Hz}, 2 \mathrm{H}), 1.96(\mathrm{~s}, 3 \mathrm{H}), 1.43(\mathrm{~s}, 6 \mathrm{H}), 1.26(\mathrm{~s}, 3 \mathrm{H}) .{ }^{13} \mathrm{C}$ NMR $(126$ $\left.\mathrm{MHz}, \mathrm{CDCl}_{3}\right) \delta 174.5,168.4,163.2,72.6,61.4,49.3,23.0,14.0,12.8$. HRMS (ESITOF) m/z: [M-H] $]^{-}$calculated for $\mathrm{C}_{17} \mathrm{H}_{16} \mathrm{~F}_{7} \mathrm{~N}_{2} \mathrm{O}_{4}{ }^{-} 445.0999$, found 445.1009 .<smiles>CCOC(=O)C(C)(Cc1ccccc1)/C(C)=N/OCC(=O)Nc1c(F)c(F)c(C(F)(F)F)c(F)c1F</smiles>

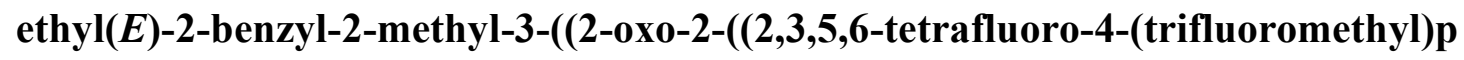
henyl)amino)ethoxy)imino)butanoate (1g)

White solid (553.3 mg, 53\%). ${ }^{1} \mathrm{H}$ NMR (500 MHz, $\left.\mathrm{CDCl}_{3}\right) \delta 7.66$ (br s, 1H), 7.16 (t, $J$ 
$=7.6 \mathrm{~Hz}, 2 \mathrm{H}), 7.05(\mathrm{~d}, J=7.2 \mathrm{~Hz}, 2 \mathrm{H}), 6.98(\mathrm{~s}, 1 \mathrm{H}), 4.68(\mathrm{~d}, J=7.1 \mathrm{~Hz}, 2 \mathrm{H}), 4.19(\mathrm{~d}$, $J=7.1 \mathrm{~Hz}, 2 \mathrm{H}), 3.23(\mathrm{~d}, J=13.9 \mathrm{~Hz}, 1 \mathrm{H}), 3.12(\mathrm{~d}, J=13.9 \mathrm{~Hz}, 1 \mathrm{H}), 2.00(\mathrm{~s}, 3 \mathrm{H}), 1.32$ $(\mathrm{s}, 3 \mathrm{H}), 1.25(\mathrm{~s}, 3 \mathrm{H}) .{ }^{13} \mathrm{C} \mathrm{NMR}\left(126 \mathrm{MHz}, \mathrm{CDCl}_{3}\right) \delta 173.6,168.5,161.9,136.4,130.4$, 128.0, 126.5, 72.5, 61.6, 54.1, 40.93, 20.0, 14.1, 13.8. HRMS (ESI-TOF) m/z: [M+H] ${ }^{+}$ calculated for $\mathrm{C}_{23} \mathrm{H}_{22} \mathrm{~F}_{7} \mathrm{~N}_{2} \mathrm{O}_{4}{ }^{+}$523.1468, found 523.1461.<smiles>C=C(C)/C(C)=N\OCC(=O)Nc1c(F)c(F)c(C(F)(F)F)c(F)c1F</smiles>

(E)-2-(((3-methylbut-3-en-2-ylidene)amino)oxy)-N-(2,3,5,6-tetrafluoro-4(trifluoromethyl)phenyl)acetamide (1h)

White solid (297.6 mg, 40\%). ${ }^{1} \mathrm{H}$ NMR (500 MHz, $\left.\mathrm{CDCl}_{3}\right) \delta 7.99$ (br s, 1H), 5.44 (d, $J$ $=47.4 \mathrm{~Hz}, 2 \mathrm{H}), 4.79(\mathrm{~s}, 2 \mathrm{H}), 2.13(\mathrm{~s}, 3 \mathrm{H}), 1.95(\mathrm{~s}, 3 \mathrm{H}) .{ }^{13} \mathrm{C} \mathrm{NMR}\left(126 \mathrm{MHz}, \mathrm{CDCl}_{3}\right) \delta$ 168.4, 160.0, 140.3, 119.3, 72.8, 18.9, 11.0. HRMS (ESI-TOF) $\mathrm{m} / \mathrm{z}:[\mathrm{M}+\mathrm{H}]^{+}$calculated for $\mathrm{C}_{14} \mathrm{H}_{12} \mathrm{~F}_{7} \mathrm{~N}_{2} \mathrm{O}_{2}{ }^{+}$373.0787, found 373.0781.<smiles>C/C(=N\OCC(=O)Nc1c(F)c(F)c(C(F)(F)F)c(F)c1F)C1CCC1</smiles>

\section{(E)-2-(((1-cyclobutylethylidene)amino)oxy)-N-(2,3,5,6-tetrafluoro-4-}

(trifluoromethyl)phenyl)acetamide (1i)

White solid (231.6 mg, 30\%). ${ }^{1} \mathrm{H}$ NMR (500 MHz, $\left.\mathrm{CDCl}_{3}\right) \delta 8.15$ (br s, $\left.1 \mathrm{H}\right), 4.68$ (s, $2 \mathrm{H}), 3.11(\mathrm{p}, J=8.6 \mathrm{~Hz}, 1 \mathrm{H}), 2.15(\mathrm{td}, J=8.5,6.1 \mathrm{~Hz}, 4 \mathrm{H}), 2.05-1.95(\mathrm{~m}, 1 \mathrm{H}), 1.90$ (s, $3 \mathrm{H}), 1.83(\mathrm{dt}, J=11.8,5.7 \mathrm{~Hz}, 1 \mathrm{H}) .{ }^{13} \mathrm{C} \mathrm{NMR}\left(126 \mathrm{MHz}, \mathrm{CDCl}_{3}\right) \delta 168.7,164.1,72.3$, 40.0, 25.6, 18.2, 12.7. HRMS (ESI-TOF) m/z: [M-H] calculated for $\mathrm{C}_{15} \mathrm{H}_{12} \mathrm{~F}_{7} \mathrm{~N}_{2} \mathrm{O}_{2}{ }^{-}$ 385.0787 , found 385.0794 .<smiles>CCOC(=O)C/C(=N\OCC(=O)Nc1c(F)c(F)c(C(F)(F)F)c(F)c1F)C(C)(C)C</smiles>

(E)-4,4-dimethyl-3-((2-oxo-2-((2,3,5,6-tetrafluoro-4-(trifluoromethyl)phenyl)amin 
o)ethoxy)imino)pentanoate ethyl (1j)

White solid (496.8 mg, 54\%). ${ }^{1} \mathrm{H}$ NMR (500 MHz, $\left.\mathrm{CDCl}_{3}\right) \delta 9.24$ (br s, $\left.1 \mathrm{H}\right), 4.77$ (s, 2H), $4.20(\mathrm{~d}, J=7.1 \mathrm{~Hz}, 2 \mathrm{H}), 3.48(\mathrm{~s}, 2 \mathrm{H}), 1.32-1.29(\mathrm{~m}, 3 \mathrm{H}), 1.16(\mathrm{~s}, 9 \mathrm{H}) .{ }^{13} \mathrm{C} \mathrm{NMR}$ $\left(126 \mathrm{MHz}, \mathrm{CDCl}_{3}\right) \delta 170.4,169.3,162.2,72.7,62.0,37.4,31.3,26.9,13.9$. HRMS (ESI-TOF) m/z: [M-H] calculated for $\mathrm{C}_{18} \mathrm{H}_{18} \mathrm{~F}_{7} \mathrm{~N}_{2} \mathrm{O}_{4}{ }^{-} 459.1155$, found 459.1168 .<smiles>CCC/C(=N\OCC(=O)Nc1c(F)c(F)c(C(F)(F)F)c(F)c1F)C(C)(C)C</smiles>

(E)-2-(((2,2-dimethylhexan-3-ylidene)amino)oxy)-N-(2,3,5,6-tetrafluoro-4-(trifluo romethyl)phenyl)acetamide $(1 \mathrm{k})$

White solid (582.4 mg, 70\%). ${ }^{1} \mathrm{H}$ NMR (500 MHz, $\left.\mathrm{CDCl}_{3}\right) \delta 8.13$ (br s, $\left.1 \mathrm{H}\right), 4.66(\mathrm{~s}$, 2H), 2.35-2.27 (m, 2H), 1.64-1.54 (m, 2H), $1.15(\mathrm{~s}, 9 \mathrm{H}), 1.00(\mathrm{t}, J=7.4 \mathrm{~Hz}, 3 \mathrm{H}) .{ }^{13} \mathrm{C}$ NMR $\left(126 \mathrm{MHz}, \mathrm{CDCl}_{3}\right) \delta 171.8,168.9,72.4,37.9,28.9,27.7,27.6,20.6,14.9$. HRMS (ESI-TOF) $\mathrm{m} / \mathrm{z}$ : $[\mathrm{M}+\mathrm{H}]^{+}$calculated for $\mathrm{C}_{17} \mathrm{H}_{20} \mathrm{~F}_{7} \mathrm{~N}_{2} \mathrm{O}_{2}{ }^{+}$417.1413, found 417.1405.<smiles>CC(C)(C)/C(CCCCCl)=N\OCC(=O)Nc1c(F)c(F)c(C(F)(F)F)c(F)c1F</smiles>

(E)-2-(((7-chloro-2,2-dimethylheptan-3-ylidene)amino)oxy)-N-(2,3,5,6-tetrafluoro -4-(trifluoromethyl)phenyl)acetamide (11)

White solid (603.2 mg, 65\%). ${ }^{1} \mathrm{H}$ NMR $\left(500 \mathrm{MHz}, \mathrm{CDCl}_{3}\right) \delta 8.11$ (br s, 1H), $4.67(\mathrm{~s}$, $2 \mathrm{H}), 3.57(\mathrm{t}, J=5.0 \mathrm{~Hz}, 2 \mathrm{H}), 2.35(\mathrm{dd}, J=8.2,4.8 \mathrm{~Hz}, 2 \mathrm{H}), 1.85(\mathrm{td}, J=10.7,5.3 \mathrm{~Hz}$, $2 \mathrm{H}), 1.73(\mathrm{dt}, J=15.8,6.1 \mathrm{~Hz}, 2 \mathrm{H}), 1.16(\mathrm{~s}, 9 \mathrm{H}) .{ }^{13} \mathrm{C} \mathrm{NMR}\left(126 \mathrm{MHz}, \mathrm{CDCl}_{3}\right) \delta 171.2$, 168.6, 120.1, 72.4, 63.6, 44.3, 37.9, 33.4, 32.9, 31.9, 29.2, 27.6, 26.1, 25.8, 24.1, 22.3. HRMS (ESI-TOF) m/z: $[\mathrm{M}+\mathrm{H}]^{+}$calculated for $\mathrm{C}_{18} \mathrm{H}_{21} \mathrm{ClF}_{7} \mathrm{~N}_{2} \mathrm{O}_{2}{ }^{+} 465.1180$, found 465.1174 . 
<smiles>CC(C)(C)/C(=N\OCC(=O)Nc1c(F)c(F)c(C(F)(F)F)c(F)c1F)c1ccccc1</smiles>

(Z)-2-(((2,2-dimethyl-1-phenylpropylidene)amino)oxy)-N-(2,3,5,6-tetrafluoro-4-(t rifluoromethyl)phenyl)acetamide (1m)

White solid (522.0 mg, 58\%). ${ }^{1} \mathrm{H}$ NMR (500 MHz, $\left.\mathrm{CDCl}_{3}\right) \delta 7.68$ (br s, 1H), 7.47-7.35 (m, 3H), 7.13-7.04 (m, 2H), 4.65 (s, 2H), 1.20 (s, 9H). $\left.{ }^{13} \mathrm{C} \mathrm{NMR} \mathrm{(126} \mathrm{MHz,} \mathrm{CDCl}_{3}\right) \delta$ 170.5, 168.0, 133.5, 128.4, 126.8, 72.6, 37.8, 28.1. HRMS (ESI-TOF) m/z: [M-H] calculated for $\mathrm{C}_{20} \mathrm{H}_{16} \mathrm{~F}_{7} \mathrm{~N}_{2} \mathrm{O}_{2}{ }^{-} 449.1100$, found 449.1112 .<smiles>COc1cccc(/C(=N\OCC(=O)Nc2c(F)c(F)c(C(F)(F)F)c(F)c2F)C(C)(C)C)c1</smiles>

(Z)-2-(((1-(3-methoxyphenyl)-2,2-dimethylpropylidene)amino)oxy)-N-(2,3,5,6tetrafluoro-4-(trifluoromethyl)phenyl)acetamide (1n)

White solid (528.0 mg, 55\%). ${ }^{1} \mathrm{H}$ NMR (500 MHz, $\left.\mathrm{CDCl}_{3}\right) \delta 7.72$ (br s, $\left.1 \mathrm{H}\right), 7.36(\mathrm{t}, J$ $=7.9 \mathrm{~Hz}, 1 \mathrm{H}), 6.93(\mathrm{dd}, J=8.3,2.1 \mathrm{~Hz}, 1 \mathrm{H}), 6.66(\mathrm{~d}, J=7.6 \mathrm{~Hz}, 1 \mathrm{H}), 6.64-6.59(\mathrm{~m}$, $1 \mathrm{H}), 4.66(\mathrm{~s}, 2 \mathrm{H}), 3.82(\mathrm{~s}, 3 \mathrm{H}), 1.21(\mathrm{~s}, 9 \mathrm{H}) .{ }^{13} \mathrm{C} \mathrm{NMR}\left(126 \mathrm{MHz}, \mathrm{CDCl}_{3}\right) \delta 170.2$, $168.0,159.5,134.8,129.6,119.0,113.4,113.0,72.6,55.3,37.8,28.1$. HRMS (ESITOF) m/z: [M-H] $]^{-}$calculated for $\mathrm{C}_{21} \mathrm{H}_{18} \mathrm{~F}_{7} \mathrm{~N}_{2} \mathrm{O}_{3}{ }^{-} 479.1206$, found 479.1215 .<smiles>Cc1ccc(/C(=N/OCC(=O)Nc2c(F)c(F)c(C(F)(F)F)c(F)c2F)C(C)(C)C)cc1</smiles>

(Z)-2-(((2,2-dimethyl-1-(p-tolyl)propylidene)amino)oxy)-N-(2,3,5,6-tetrafluoro-4(trifluoromethyl)phenyl)acetamide (10)

White solid (464.0 mg, 50\%). ${ }^{1} \mathrm{H}$ NMR (500 MHz, $\left.\mathrm{CDCl}_{3}\right) \delta 7.75$ (br s, 1H), 7.26 (d, $J$ $=7.6 \mathrm{~Hz}, 2 \mathrm{H}), 6.98(\mathrm{~d}, J=7.9 \mathrm{~Hz}, 2 \mathrm{H}), 4.65(\mathrm{~s}, 2 \mathrm{H}), 2.39(\mathrm{~s}, 3 \mathrm{H}), 1.20(\mathrm{~s}, 9 \mathrm{H}) .{ }^{13} \mathrm{C}$ NMR (126 MHz, $\left.\mathrm{CDCl}_{3}\right) \delta 170.7,168.2,138.3,130.4,129.1,126.7,72.6,37.9,28.1$, 
21.3. HRMS (ESI-TOF) m/z: [M-H] calculated for $\mathrm{C}_{21} \mathrm{H}_{18} \mathrm{~F}_{7} \mathrm{~N}_{2} \mathrm{O}_{2}^{-} 463.1257$, found 463.1267.<smiles>CC(C)(C)/C(=N\OCC(=O)Nc1c(F)c(F)c(C(F)(F)F)c(F)c1F)c1ccc(C(C)(C)C)cc1</smiles>

(Z)-2-(((1-(4-(tert-butyl)phenyl)-2,2-dimethylpropylidene)amino)oxy)-N-(2,3,5,6tetrafluoro-4-(trifluoromethyl)phenyl)acetamide (1p)

White solid (526.2 mg, 52\%). ${ }^{1} \mathrm{H}$ NMR (500 MHz, $\left.\mathrm{CDCl}_{3}\right) \delta 7.73$ (br s, 1H), 7.44 (d, $J$ $=8.4 \mathrm{~Hz}, 2 \mathrm{H}), 7.01(\mathrm{~d}, J=8.4 \mathrm{~Hz}, 2 \mathrm{H}), 4.65(\mathrm{~s}, 2 \mathrm{H}), 1.34(\mathrm{~s}, 9 \mathrm{H}), 1.19(\mathrm{~s}, 9 \mathrm{H}) .{ }^{13} \mathrm{C}$ NMR $\left(126 \mathrm{MHz}, \mathrm{CDCl}_{3}\right) \delta 170.7,168.1,151.3,130.5,126.5,125.3,72.5,37.9,34.7$, 31.3, 28.1. HRMS (ESI-TOF) m/z: [M-H] $]^{-}$calculated for $\mathrm{C}_{24} \mathrm{H}_{24} \mathrm{~F}_{7} \mathrm{~N}_{2} \mathrm{O}_{2}{ }^{-}$505.1726, found 505.1740 .<smiles>CC(C)(C)/C(=N\OCC(=O)Nc1c(F)c(F)c(C(F)(F)F)c(F)c1F)c1ccc(F)cc1</smiles>

(Z)-2-(((1-(4-fluorophenyl)-2,2-dimethylpropylidene)amino)oxy)-N-(2,3,5,6tetrafluoro-4-(trifluoromethyl)phenyl)acetamide (1q)

White solid (526.3 mg, 49\%). ${ }^{1} \mathrm{H}$ NMR (500 MHz, $\left.\mathrm{CDCl}_{3}\right) \delta 7.68$ (br s, 1H), 7.14 (dt, $J=8.9,1.7 \mathrm{~Hz}, 2 \mathrm{H}), 7.10-7.03(\mathrm{~m}, 2 \mathrm{H}), 4.65$ (s, 2H), 1.19 (s, 9H). ${ }^{13} \mathrm{C} \mathrm{NMR}(126 \mathrm{MHz}$, $\left.\mathrm{CDCl}_{3}\right) \delta 169.7,167.8,163.5,161.5,129.2,129.2,128.8,128.8,115.7,115.5,72.6$, 37.9, 28.1. HRMS (ESI-TOF) m/z: [M-H] calculated for $\mathrm{C}_{20} \mathrm{H}_{15} \mathrm{~F}_{8} \mathrm{~N}_{2} \mathrm{O}_{2}{ }^{-}$467.1006, found 467.1014 .<smiles>CC(C)(C)/C(=N\OCC(=O)Nc1c(F)c(F)c(C(F)(F)F)c(F)c1F)c1ccc(Cl)cc1</smiles>

(Z)-2-(((1-(4-chlorophenyl)-2,2-dimethylpropylidene)amino)oxy)-N-(2,3,5,6- 
White solid (580.8 mg, 60\%). ${ }^{1} \mathrm{H}$ NMR $\left(500 \mathrm{MHz}, \mathrm{CDCl}_{3}\right) \delta 7.68$ (br s, $\left.1 \mathrm{H}\right), 7.42$ (d, $J$ $=8.4 \mathrm{~Hz}, 2 \mathrm{H}), 7.04(\mathrm{~d}, J=8.4 \mathrm{~Hz}, 2 \mathrm{H}), 4.64(\mathrm{~s}, 2 \mathrm{H}), 1.20(\mathrm{~s}, 9 \mathrm{H}) .{ }^{13} \mathrm{C} \mathrm{NMR}(126 \mathrm{MHz}$, $\left.\mathrm{CDCl}_{3}\right) \delta 169.5,167.7,134.6,131.7,128.8,128.4,72.7,37.8,28.0$. HRMS (ESI-TOF) $\mathrm{m} / \mathrm{z}$ : [M-H] ${ }^{-}$calculated for $\mathrm{C}_{20} \mathrm{H}_{15} \mathrm{ClF}_{7} \mathrm{~N}_{2} \mathrm{O}_{2}{ }^{-} 483.0711$, found 483.0723 .<smiles>COc1ccc(/C(=N/OCC(=O)Nc2c(F)c(F)c(C(F)(F)F)c(F)c2F)C(C)(C)C)cc1</smiles>

(Z)-2-(((1-(4-methoxyphenyl)-2,2-dimethylpropylidene)amino)oxy)-N-(2,3,5,6-tet rafluoro-4-(trifluoromethyl)phenyl)acetamide (1s)

White solid (547.1 mg, 57\%). ${ }^{1} \mathrm{H}$ NMR (500 MHz, $\left.\mathrm{CDCl}_{3}\right) \delta 7.75$ (br s, 1H), 7.03 (s, $2 \mathrm{H}), 6.99(\mathrm{~s}, 2 \mathrm{H}), 4.67(\mathrm{~s}, 2 \mathrm{H}), 3.86(\mathrm{~s}, 3 \mathrm{H}), 1.22(\mathrm{~s}, 9 \mathrm{H}) .{ }^{13} \mathrm{C} \mathrm{NMR}\left(126 \mathrm{MHz}, \mathrm{CDCl}_{3}\right)$ $\delta 170.4,168.1,159.5,128.2,125.5,113.9,72.6,55.2,37.9,28.1$. HRMS (ESI-TOF) $\mathrm{m} / \mathrm{z}$ : [M-H] $]^{-}$calculated for $\mathrm{C}_{21} \mathrm{H}_{18} \mathrm{~F}_{7} \mathrm{~N}_{2} \mathrm{O}_{3}{ }^{-} 479.1206$, found 479.1216 .<smiles>CC(C)(C)/C(=N\OCC(=O)Nc1c(F)c(F)c(C(F)(F)F)c(F)c1F)c1cccs1</smiles>

(Z)-2-(((2,2-dimethyl-1-(thiophen-2-yl)propylidene)amino)oxy)-N-(2,3,5,6tetrafluoro-4-(trifluoromethyl)phenyl)acetamide (1t)

White solid (538.1 mg, 59\%). ${ }^{1} \mathrm{H}$ NMR $\left(500 \mathrm{MHz}, \mathrm{CDCl}_{3}\right) \delta 7.81$ (br s, $\left.1 \mathrm{H}\right), 7.49$ (dd, $J=5.0,0.9 \mathrm{~Hz}, 1 \mathrm{H}), 7.14(\mathrm{dd}, J=5.0,3.7 \mathrm{~Hz}, 1 \mathrm{H}), 7.10(\mathrm{dd}, J=3.5,0.9 \mathrm{~Hz}, 1 \mathrm{H}), 4.76$ $(\mathrm{s}, 2 \mathrm{H}), 1.28(\mathrm{~s}, 9 \mathrm{H}) .{ }^{13} \mathrm{C} \mathrm{NMR}\left(126 \mathrm{MHz}, \mathrm{CDCl}_{3}\right) \delta 167.7,163.4,130.5,127.9,127.2$, 126.8, 72.9, 38.3, 28.4. HRMS (ESI-TOF) $\mathrm{m} / \mathrm{z}$ : $[\mathrm{M}+\mathrm{H}]^{+}$calculated for $\mathrm{C}_{18} \mathrm{H}_{16} \mathrm{~F}_{7} \mathrm{~N}_{2} \mathrm{O}_{2} \mathrm{~S}^{+}$ 457.0820 , found 457.0813 .<smiles>CC(C)(C)C(/C=C/c1ccccc1)=N\OCC(=O)Nc1c(F)c(F)c(C(F)(F)F)c(F)c1F</smiles> 
2-((((1E,3E)-4,4-dimethyl-1-phenylpent-1-en-3-ylidene)amino)oxy)-N-(2,3,5,6-tet rafluoro-4-(trifluoromethyl)phenyl)acetamide (1u)

White solid (647.3 mg, 68\%). ${ }^{1} \mathrm{H}$ NMR (500 MHz, $\left.\mathrm{CDCl}_{3}\right) \delta 8.08$ (br s, 1H), 7.52 (d, $J$ $=7.2 \mathrm{~Hz}, 2 \mathrm{H}), 7.42(\mathrm{dd}, J=12.3,5.3 \mathrm{~Hz}, 3 \mathrm{H}), 7.39-7.36(\mathrm{~m}, 1 \mathrm{H}), 6.70(\mathrm{~d}, J=16.8 \mathrm{~Hz}$, $1 \mathrm{H}), 4.80(\mathrm{~s}, 2 \mathrm{H}), 1.29(\mathrm{~s}, 9 \mathrm{H}) .{ }^{13} \mathrm{C} \mathrm{NMR}(126 \mathrm{MHz}, \mathrm{CDCl} 3) \delta 168.5,165.0,140.3$, 136.2, 129.3, 128.9, 127.0, 116.2, 72.8, 38.0, 28.7. HRMS (ESI-TOF) m/z: $[\mathrm{M}+\mathrm{H}]^{+}$ calculated for $\mathrm{C}_{22} \mathrm{H}_{20} \mathrm{~F}_{7} \mathrm{~N}_{2} \mathrm{O}_{2}{ }^{+} 477.1413$, found 477.1409 .<smiles>CC(C)(C)/C(C=Cc1ccccc1[N+](=O)[O-])=N\OCC(=O)Nc1c(F)c(F)c(C(F)(F)F)c(F)c1F</smiles>

2-((((1E,3E)-4,4-dimethyl-1-(2-nitrophenyl)pent-1-en-3-ylidene)amino)oxy)-N(2,3,5,6-tetrafluoro-4-(trifluoromethyl)phenyl)acetamide (1v)

White solid (635.5 mg, 61\%). ${ }^{1} \mathrm{H}$ NMR (500 MHz, $\left.\mathrm{CDCl}_{3}\right) \delta 8.08$ (br s, $\left.1 \mathrm{H}\right), 8.04$ (d, $J$ $=8.1 \mathrm{~Hz}, 1 \mathrm{H}), 7.75(\mathrm{~d}, J=16.7 \mathrm{~Hz}, 1 \mathrm{H}), 7.72-7.61(\mathrm{~m}, 2 \mathrm{H}), 7.55-7.48(\mathrm{~m}, 1 \mathrm{H}), 6.57$ $(\mathrm{d}, J=16.7 \mathrm{~Hz}, 1 \mathrm{H}), 4.80(\mathrm{~s}, 2 \mathrm{H}), 1.28(\mathrm{~s}, 9 \mathrm{H}) .{ }^{13} \mathrm{C} \mathrm{NMR}\left(126 \mathrm{MHz}, \mathrm{CDCl}_{3}\right) \delta 168.1$, 164.3, 147.9, 135.1, 133.8, 132.8, 129.5, 128.9, 125.0, 121.6, 73.1, 37.9, 28.5. HRMS (ESI-TOF) m/z: $[\mathrm{M}+\mathrm{H}]^{+}$calculated for $\mathrm{C}_{22} \mathrm{H}_{19} \mathrm{~F}_{7} \mathrm{~N}_{3} \mathrm{O}_{4}{ }^{+}$522.1264, found 522.1259.<smiles>CC(C)(C)C(/C=C/c1cccc(C#N)c1)=N\OCC(=O)Nc1c(F)c(F)c(C(F)(F)F)c(F)c1F</smiles>

2-((((1E,3E)-1-(3-cyanophenyl)-4,4-dimethylpent-1-en-3-ylidene)amino)oxy)-N(2,3,5,6-tetrafluoro-4-(trifluoromethyl)phenyl)acetamide (1w)

White solid (480.6 mg, 48\%). ${ }^{1} \mathrm{H}$ NMR (500 MHz, $\mathrm{CDCl}_{3}$ ) $\delta 7.97$ (br s, 1H), 7.77 (s, $1 \mathrm{H}), 7.70(\mathrm{~d}, J=7.9 \mathrm{~Hz}, 1 \mathrm{H}), 7.62(\mathrm{~d}, J=7.7 \mathrm{~Hz}, 1 \mathrm{H}), 7.51(\mathrm{t}, J=7.8 \mathrm{~Hz}, 1 \mathrm{H}), 7.37$ (d, $J=16.9 \mathrm{~Hz}, 1 \mathrm{H}), 6.69(\mathrm{~d}, J=16.9 \mathrm{~Hz}, 1 \mathrm{H}), 4.78(\mathrm{~s}, 2 \mathrm{H}), 1.27$ (s, 9H). ${ }^{13} \mathrm{C}$ NMR $(126$ $\left.\mathrm{MHz}, \mathrm{CDCl}_{3}\right) \delta 168.2,164.4,137.6,137.5,132.2,131.1,130.3,129.8,118.7,118.4$, 113.3, 72.9, 38.0, 28.6. HRMS (ESI-TOF) $\mathrm{m} / \mathrm{z}$ : $[\mathrm{M}+\mathrm{H}]^{+}$calculated for $\mathrm{C}_{23} \mathrm{H}_{19} \mathrm{~F}_{7} \mathrm{~N}_{3} \mathrm{O}_{2}{ }^{+}$ 502.1365 , found 502.1358 . 
<smiles>CC(C)(C)C(/C=C/c1ccc([N+](=O)[O-])cc1)=N\OCC(=O)Nc1c(F)c(F)c(C(F)(F)F)c(F)c1F</smiles>

2-((((1E,3E)-4,4-dimethyl-1-(4-nitrophenyl)pent-1-en-3-ylidene)amino)oxy)-N(2,3,5,6-tetrafluoro-4-(trifluoromethyl)phenyl)acetamide (1x)

pale yellow solid (510.6 mg, 49\%). ${ }^{1} \mathrm{H} \mathrm{NMR}\left(500 \mathrm{MHz}, \mathrm{CDCl}_{3}\right) \delta 8.23(\mathrm{~d}, J=8.7 \mathrm{~Hz}$, 2H), 7.98 (br s, 1H), 7.63 (d, $J=8.7 \mathrm{~Hz}, 2 \mathrm{H}), 7.43$ (d, $J=16.8 \mathrm{~Hz}, 1 \mathrm{H}), 6.79$ (d, $J=$ $16.9 \mathrm{~Hz}, 1 \mathrm{H}), 4.79(\mathrm{~s}, 2 \mathrm{H}), 1.28(\mathrm{~s}, 9 \mathrm{H}) .{ }^{13} \mathrm{C} \mathrm{NMR}\left(126 \mathrm{MHz}, \mathrm{CDCl}_{3}\right) \delta 168.0,164.3$, 147.9, 142.4, 137.4, 127.6, 124.2, 120.5, 73.0, 38.0, 28.6. HRMS (ESI-TOF) m/z: $[\mathrm{M}+\mathrm{H}]^{+}$calculated for $\mathrm{C}_{22} \mathrm{H}_{19} \mathrm{~F}_{7} \mathrm{~N}_{3} \mathrm{O}_{4}{ }^{+}$522.1264, found 522.1259 .<smiles>CC(C)(C)C(/C=C/c1ccc(F)cc1)=N\OCC(=O)Nc1c(F)c(F)c(C(F)(F)F)c(F)c1F</smiles>

2-((((1E,3E)-1-(4-fluorophenyl)-4,4-dimethylpent-1-en-3-ylidene)amino)oxy)-N(2,3,5,6-tetrafluoro-4-(trifluoromethyl)phenyl)acetamide (1y)

White solid (632.3 mg, 64\%). ${ }^{1} \mathrm{H}$ NMR (500 MHz, $\left.\mathrm{CDCl}_{3}\right) \delta 8.05$ (br s, 1H), 7.47 (dd, $J=8.3,5.5 \mathrm{~Hz}, 2 \mathrm{H}), 7.41(\mathrm{~d}, J=16.8 \mathrm{~Hz}, 1 \mathrm{H}), 7.08(\mathrm{t}, J=8.5 \mathrm{~Hz}, 2 \mathrm{H}), 6.59$ (d, $J=$ $16.8 \mathrm{~Hz}, 1 \mathrm{H}), 4.77$ (s, 2H), 1.27 (s, 9H). ${ }^{13} \mathrm{C} \mathrm{NMR}\left(126 \mathrm{MHz}, \mathrm{CDCl}_{3}\right) \delta 168.4,164.8$, 164.3, 162.3, 139.1, 132.4, 128.8, 128.7, 116.1, 115.9, 72.8, 38.0, 28.7. HRMS (ESITOF) m/z: $[\mathrm{M}+\mathrm{H}]^{+}$calculated for $\mathrm{C}_{22} \mathrm{H}_{19} \mathrm{~F}_{8} \mathrm{~N}_{2} \mathrm{O}_{2}{ }^{+} 495.1319$, found 495.1311 .<smiles>CC1=C2[C@H]3OC(=O)[C@H](C)[C@@H]3CC[C@]2(C)C=C/C1=N\OCC(=O)Nc1c(F)c(F)c(C(F)(F)F)c(F)c1F</smiles>

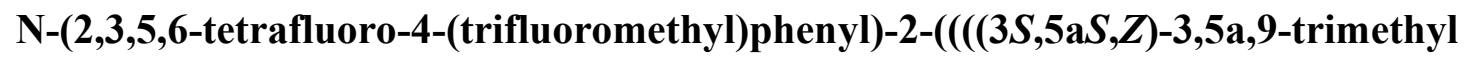
-2-oxo-2,3-dihydro-3al5-naphtho[1,2-b]furan-8(5aH)-ylidene)amino)oxy)acetami de (1z)

Yellow solid (448.5 mg, 42\%). ${ }^{1} \mathrm{H}$ NMR $\left(500 \mathrm{MHz}, \mathrm{CDCl}_{3}\right) \delta 8.13$ (br s, $\left.1 \mathrm{H}\right), 6.90$ (d, $J=10.1 \mathrm{~Hz}, 1 \mathrm{H}), 6.19(\mathrm{~d}, J=10.1 \mathrm{~Hz}, 1 \mathrm{H}), 4.79$ (s, 3H), 2.37 (dd, $J=12.2,6.9 \mathrm{~Hz}$, $1 \mathrm{H}), 2.17(\mathrm{~d}, J=0.9 \mathrm{~Hz}, 3 \mathrm{H}), 2.02(\mathrm{~d}, J=12.3 \mathrm{~Hz}, 1 \mathrm{H}), 1.83-1.78(\mathrm{~m}, 2 \mathrm{H}), 1.68(\mathrm{dd}, J$ 
$=12.6,3.7 \mathrm{~Hz}, 1 \mathrm{H}), 1.53-1.49(\mathrm{~m}, 1 \mathrm{H}), 1.28(\mathrm{~d}, J=2.9 \mathrm{~Hz}, 6 \mathrm{H}) .{ }^{13} \mathrm{C}$ NMR $(126 \mathrm{MHz}$, $\left.\mathrm{CDCl}_{3}\right) \delta 177.8,168.4,153.2,148.0,141.4,122.2,112.3,82.0,72.9,53.5,41.2,41.0$, 38.1, 25.6, 23.6, 12.5, 12.0. HRMS (ESI-TOF) $\mathrm{m} / \mathrm{z}:[\mathrm{M}+\mathrm{H}]^{+}$calculated for $\mathrm{C}_{24} \mathrm{H}_{22} \mathrm{~F}_{7} \mathrm{~N}_{2} \mathrm{O}_{4}{ }^{+}$535.1468, found 535.1466. 


\section{Optimization of $\mathrm{C}\left(\mathrm{sp}^{3}\right)-\mathrm{H}$ Silylation ${ }^{a}$}<smiles>CCC(C)(C)/C(C)=N\OCC(=O)NC(F)F</smiles>

$$
\underset{\text { solvent, temperature }}{\stackrel{\begin{array}{c}
\text { catalyst, additive } \\
\text { oxidant, base }
\end{array}}{\longrightarrow}}
$$

\begin{tabular}{|c|c|c|c|c|c|c|c|}
\hline entry & catalyst & oxidant & solvent & additive & base & $\mathrm{T}\left({ }^{\circ} \mathrm{C}\right)$ & yield $(\%)^{k}$ \\
\hline 1 & $\mathrm{Pd}(\mathrm{OAc})_{2}$ & $\mathrm{AgOAc}$ & DCE & I & $\mathrm{Na}_{2} \mathrm{CO}_{3}$ & $100^{\circ} \mathrm{C}$ & $13 \%$ \\
\hline 2 & $\mathrm{Pd}(\mathrm{OAc})_{2}$ & $\mathrm{AgOAc}$ & Toluene & I & $\mathrm{Na}_{2} \mathrm{CO}_{3}$ & $100^{\circ} \mathrm{C}$ & $20 \%$ \\
\hline 3 & $\mathrm{Pd}(\mathrm{OAc})_{2}$ & $\mathrm{AgOAc}$ & HFIP & I & $\mathrm{Na}_{2} \mathrm{CO}_{3}$ & $100{ }^{\circ} \mathrm{C}$ & n.d. \\
\hline 4 & $\mathrm{Pd}(\mathrm{OAc})_{2}$ & $\mathrm{AgOAc}$ & Toluene & 1 & $\mathrm{Na}_{2} \mathrm{CO}_{3}$ & $90^{\circ} \mathrm{C}$ & $10 \%$ \\
\hline 5 & $\mathrm{Pd}(\mathrm{OAc})_{2}$ & $\mathrm{AgOAc}$ & Toluene & 1 & $\mathrm{Na}_{2} \mathrm{CO}_{3}$ & $110^{\circ} \mathrm{C}$ & $28 \%$ \\
\hline 6 & $\mathrm{Pd}(\mathrm{OAc})_{2}$ & $\mathrm{AgOAc}$ & Toluene & I & $\mathrm{Na}_{2} \mathrm{CO}_{3}$ & $120^{\circ} \mathrm{C}$ & $22 \%$ \\
\hline 7 & $\mathrm{Pd}(\mathrm{OAc})_{2}$ & AgTFA & Toluene & I & $\mathrm{Na}_{2} \mathrm{CO}_{3}$ & $110^{\circ} \mathrm{C}$ & $41 \%$ \\
\hline 8 & $\mathrm{Pd}(\mathrm{OAc})_{2}$ & $\mathrm{Ag}_{2} \mathrm{CO}_{3}$ & Toluene & I & $\mathrm{Na}_{2} \mathrm{CO}_{3}$ & $110^{\circ} \mathrm{C}$ & $5 \%$ \\
\hline 9 & $\mathrm{Pd}(\mathrm{CN})_{2} \mathrm{Cl}_{2}$ & AgTFA & Toluene & I & $\mathrm{Na}_{2} \mathrm{CO}_{3}$ & $110^{\circ} \mathrm{C}$ & trace \\
\hline 10 & $\mathrm{Pd}(\text { acac })_{2}$ & AgTFA & Toluene & I & $\mathrm{Na}_{2} \mathrm{CO}_{3}$ & $110^{\circ} \mathrm{C}$ & $17 \%$ \\
\hline 11 & $\mathrm{Pd}(\mathrm{OAc})_{2}$ & AgTFA & Toluene & $\mathrm{Cu}(\mathrm{OAc})_{2} \cdot \mathrm{H}_{2} \mathrm{O}$ & $\mathrm{Na}_{2} \mathrm{CO}_{3}$ & $110^{\circ} \mathrm{C}$ & $66 \%$ \\
\hline 12 & $\mathrm{Pd}(\mathrm{OAc})_{2}$ & AgTFA & Toluene & $\mathrm{Cu}\left(\mathrm{ClO}_{4}\right)_{2}$ & $\mathrm{Na}_{2} \mathrm{CO}_{3}$ & $110^{\circ} \mathrm{C}$ & $31 \%$ \\
\hline 13 & $\mathrm{Pd}(\mathrm{OAc})_{2}$ & AgTFA & Toluene & $\mathrm{Cu}(\mathrm{OAc})_{2} \cdot \mathrm{H}_{2} \mathrm{O}$ & $\mathrm{Li}_{2} \mathrm{CO}_{3}$ & $110^{\circ} \mathrm{C}$ & $73 \%(72 \%)^{c}$ \\
\hline 14 & $\mathrm{Pd}(\mathrm{OAc})_{2}$ & AgTFA & Toluene & $\mathrm{Cu}(\mathrm{OAc})_{2} \cdot \mathrm{H}_{2} \mathrm{O}$ & $\mathrm{K}_{2} \mathrm{HPO}_{4}$ & $110^{\circ} \mathrm{C}$ & $48 \%$ \\
\hline 15 & I & AgTFA & Toluene & $\mathrm{Cu}(\mathrm{OAc})_{2} \cdot \mathrm{H}_{2} \mathrm{O}$ & $\mathrm{Li}_{2} \mathrm{CO}_{3}$ & $110^{\circ} \mathrm{C}$ & n.d. \\
\hline 16 & $\mathrm{Pd}(\mathrm{OAc})_{2}$ & I & Toluene & $\mathrm{Cu}(\mathrm{OAc})_{2} \cdot \mathrm{H}_{2} \mathrm{O}$ & $\mathrm{Li}_{2} \mathrm{CO}_{3}$ & $110^{\circ} \mathrm{C}$ & $4 \%$ \\
\hline 18 & $\mathrm{Pd}(\mathrm{OAc})_{2}$ & AgTFA & Toluene & $\mathrm{Cu}(\mathrm{OAc})_{2} \cdot \mathrm{H}_{2} \mathrm{O}$ & I & $110^{\circ} \mathrm{C}$ & $38 \%$ \\
\hline $19^{d}$ & $\mathrm{Pd}(\mathrm{OAc})_{2}$ & AgTFA & Toluene & $\mathrm{Cu}(\mathrm{OAc})_{2} \cdot \mathrm{H}_{2} \mathrm{O}$ & $\mathrm{Li}_{2} \mathrm{CO}_{3}$ & $110^{\circ} \mathrm{C}$ & $70 \%$ \\
\hline $20^{e}$ & $\mathrm{Pd}(\mathrm{OAc})_{2}$ & AgTFA & Toluene & $\mathrm{Cu}(\mathrm{OAc})_{2} \cdot \mathrm{H}_{2} \mathrm{O}$ & $\mathrm{Li}_{2} \mathrm{CO}_{3}$ & $110^{\circ} \mathrm{C}$ & $67 \%$ \\
\hline $21^{f}$ & $\mathrm{Pd}(\mathrm{OAc})_{2}$ & AgTFA & Toluene & $\mathrm{Cu}(\mathrm{OAc})_{2} \mathrm{H}_{2} \mathrm{O}$ & $\mathrm{Li}_{2} \mathrm{CO}_{3}$ & $110^{\circ} \mathrm{C}$ & $33 \%$ \\
\hline $22^{g}$ & $\mathrm{Pd}(\mathrm{OAc})_{2}$ & AgTFA & Toluene & $\mathrm{Cu}(\mathrm{OAc})_{2} \mathrm{H}_{2} \mathrm{O}$ & $\mathrm{Li}_{2} \mathrm{CO}_{3}$ & $110^{\circ} \mathrm{C}$ & $6 \%$ \\
\hline $23^{h}$ & $\mathrm{Pd}(\mathrm{OAc})_{2}$ & AgTFA & Toluene & $\mathrm{Cu}(\mathrm{OAc})_{2} \mathrm{H}_{2} \mathrm{O}$ & $\mathrm{Li}_{2} \mathrm{CO}_{3}$ & $110^{\circ} \mathrm{C}$ & $11 \%$ \\
\hline
\end{tabular}<smiles>CC(=NOCC(=O)NC(F)F)C(C)(C)C[SiH3]</smiles><smiles>COCC(=O)Nc1c(F)c(F)c(C(F)(F)F)c(F)c1F</smiles>

$\mathrm{DG}_{2}=\quad \mathrm{DG}_{3}=$<smiles>COCC(=O)O</smiles><smiles>COC(C)(C)C(=O)O</smiles><smiles>COC(C)(C)C(=O)Nc1c(F)c(F)c(C(F)(F)F)c(F)c1F</smiles>

${ }^{a}$ Conditions: 1a $(0.1 \mathrm{mmol}, 1.0$ equiv), catalyst (10 mol\%), additives (20 mol\%), 
oxidanat (3.0 equiv), $\mathrm{Li}_{2} \mathrm{CO}_{3}$ (2.0 equiv), $\left(\mathrm{SiMe}_{3}\right)_{2}$ (2.5 equiv), solvent $(2.0 \mathrm{~mL}), 110{ }^{\circ} \mathrm{C}$ in an oil bath, under air, 6 hours. ${ }^{b}$ Yield determined by ${ }^{1} \mathrm{H}$ NMR analysis of the crude product using 1,3,5-trimethoxybenzene as internal standard. ${ }^{c}$ Isolated yield. ${ }^{d}$ Under argon atmosphere. ${ }^{e}$ Under oxygen atmosphere. ${ }^{f} \mathbf{D G}_{2}$ instead of $\mathbf{D G}_{1} .{ }^{g} \mathbf{D G}_{3}$ instead of DG1. ${ }^{h} \mathbf{D G}_{4}$ instead of $\mathbf{D G}_{\mathbf{1}}$.

\section{C(sp $\left.{ }^{3}\right)-H$ Silylation}

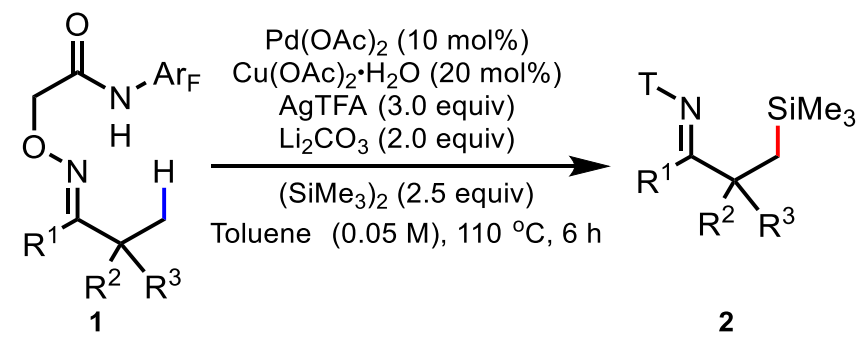

\section{General Procedures for the $\mathbf{C}\left(\mathrm{sp}^{\mathbf{3}}\right)-\mathrm{H}$ Silylation of Ketones:}

Substrate $1(0.10 \mathrm{mmol}), \mathrm{Pd}(\mathrm{OAc})_{2}(0.01 \mathrm{mmol}, 2.2 \mathrm{mg}), \mathrm{Cu}(\mathrm{OAc})_{2} \cdot \mathrm{H}_{2} \mathrm{O}(0.02 \mathrm{mmol}$, $4.0 \mathrm{mg}), \operatorname{AgTFA}(0.30 \mathrm{mmol}, 66.0 \mathrm{mg}), \mathrm{Li}_{2} \mathrm{CO}_{3}(0.20 \mathrm{mmol}, 14.8 \mathrm{mg}),\left(\mathrm{SiMe}_{3}\right)_{2}(0.25$ $\mathrm{mmol}, 73.0 \mathrm{mg})$ were weighed into a reaction vial $(10 \mathrm{~mL})$ with a magnetic stir bar under air. Toluene $(2.0 \mathrm{~mL})$ was added, and the vial was sealed with a cap. The reaction mixture was stirred at $110{ }^{\circ} \mathrm{C}$ in an oil bath for 6 hours. Upon completion, the reaction mixture was cooled to room temperature and diluted with EtOAc. Then the reaction mixture was filtered through a plug of celite and the solvent was removed under vacuum. The resulting mixture was purified by column chromatography.<smiles>CCC(C)(CC)/C(C)=N\OCC(=O)Nc1c(F)c(F)c(C(F)(F)F)c(F)c1F</smiles>

(E)-2-((3,3-dimethyl-4-(trimethylsilyl)butan-2-ylidene)amino)oxy)-N-(2,3,5,6tetrafluoro-4-(trifluoromethyl)phenyl)acetamide (2a)

Substrate 1a was silylated following the general silylation procedure. After purification by column chromatography (Hexane : EtOAc $=10: 1$ as eluent), 2a was obtained as a colorless oil (33.6 mg, 73\%). ${ }^{1} \mathrm{H}$ NMR $\left(500 \mathrm{MHz}, \mathrm{CDCl}_{3}\right) \delta 8.13$ (br s, $\left.1 \mathrm{H}\right), 4.68$ (s, 2H), 1.92 (s, 3H), 1.19 (s, 6H), 0.92 (s, 2H), 0.01 (s, 9H). ${ }^{13} \mathrm{C} \mathrm{NMR}\left(126 \mathrm{MHz}, \mathrm{CDCl}_{3}\right)$ $\delta 168.9,168.7,72.3,40.3,29.9,28.6,11.4,0.4$. HRMS (ESI-TOF) $\mathrm{m} / \mathrm{z}:[\mathrm{M}+\mathrm{H}]^{+}$ calculated for $\mathrm{C}_{18} \mathrm{H}_{24} \mathrm{~F}_{7} \mathrm{~N}_{2} \mathrm{O}_{2} \mathrm{Si}^{+} 461.1495$, found 461.1491 . 
<smiles>C/C(=N/OCC(=O)Nc1c(F)c(F)c(C(F)(F)F)c(F)c1F)C(C)(CSc1ccccc1)Cc1ccccc1</smiles>

\section{(E)-2-(((3-benzyl-3-methyl-4-(trimethylsilyl)butan-2-ylidene)amino)oxy)-N-}

(2,3,5,6-tetrafluoro-4-(trifluoromethyl)phenyl)acetamide (2b)

Substrate 1b was silylated following the general silylation procedure. After purification by column chromatography (Hexane : EtOAc $=10: 1$ as eluent), $2 \mathbf{b}$ was obtained as a white solid (40.2 mg, 75\%). ${ }^{1} \mathrm{H} \mathrm{NMR}\left(500 \mathrm{MHz}, \mathrm{CDCl}_{3}\right) \delta 7.58$ (br s, $\left.1 \mathrm{H}\right), 7.06(\mathrm{t}, J=$ $7.6 \mathrm{~Hz}, 2 \mathrm{H}), 6.98(\mathrm{~d}, J=7.4 \mathrm{~Hz}, 2 \mathrm{H}), 6.76(\mathrm{t}, J=7.3 \mathrm{~Hz}, 1 \mathrm{H}), 4.58(\mathrm{~d}, J=1.3 \mathrm{~Hz}, 2 \mathrm{H})$, $2.76(\mathrm{dd}, J=92.9,13.5 \mathrm{~Hz}, 2 \mathrm{H}), 2.05(\mathrm{~s}, 3 \mathrm{H}), 1.18(\mathrm{~s}, 3 \mathrm{H}), 1.15(\mathrm{~d}, J=14.5 \mathrm{~Hz}, 1 \mathrm{H})$, $0.86(\mathrm{~d}, J=14.5 \mathrm{~Hz}, 1 \mathrm{H}), 0.04(\mathrm{~s}, 9 \mathrm{H}) .{ }^{13} \mathrm{C} \mathrm{NMR}\left(126 \mathrm{MHz}, \mathrm{CDCl}_{3}\right) \delta 169.1,166.6$, 137.8, 130.2, 127.6, 125.8, 72.0, 49.6, 44.7, 28.6, 23.6, 12.6, 0.6. HRMS (ESI-TOF) $\mathrm{m} / \mathrm{z}$ : $[\mathrm{M}+\mathrm{H}]^{+}$calculated for $\mathrm{C}_{24} \mathrm{H}_{28} \mathrm{~F}_{7} \mathrm{~N}_{2} \mathrm{O}_{2} \mathrm{Si}^{+}$537.1808, found 537.1801.<smiles>CCC(CC)(Cc1ccccc1)/C(C)=N/OCC(=O)Nc1c(F)c(F)c(C(F)(F)F)c(F)c1[13C](=O)[O-]</smiles>

\section{(E)-2-(((3-benzyl-3-((trimethylsilyl)methyl)pentan-2-ylidene)amino)oxy)-N- (2,3,5,6-tetrafluoro-4-(trifluoromethyl)phenyl)acetamide (2c)}

Substrate 1c was silylated following the general silylation procedure. After purification by column chromatography (Hexane : EtOAc $=10: 1$ as eluent), 2c was obtained as a pale yellow oil (20.4 mg, 37\%). ${ }^{1} \mathrm{H}$ NMR $\left(500 \mathrm{MHz}, \mathrm{CDCl}_{3}\right) \delta 7.55$ (br s, 1H), 7.05 (t, $J=7.6 \mathrm{~Hz}, 3 \mathrm{H}), 7.00(\mathrm{~d}, J=7.1 \mathrm{~Hz}, 2 \mathrm{H}), 6.74(\mathrm{t}, J=7.3 \mathrm{~Hz}, 1 \mathrm{H}), 4.59(\mathrm{~s}, 2 \mathrm{H}), 2.81(\mathrm{~d}$, $J=1.6 \mathrm{~Hz}, 2 \mathrm{H}), 2.04(\mathrm{~s}, 3 \mathrm{H}), 1.64(\mathrm{~m}, 2 \mathrm{H}), 1.09-0.99(\mathrm{~m}, 2 \mathrm{H}), 0.96(\mathrm{dd}, J=9.8,5.1$ $\mathrm{Hz}, 3 \mathrm{H}), 0.07$ (s, 9H). ${ }^{13} \mathrm{C} \mathrm{NMR}\left(126 \mathrm{MHz}, \mathrm{CDCl}_{3}\right) \delta 169.0,166.7,138.0,130.0,127.7$, 125.7, 72.0, 48.3, 44.3, 27.5, 23.2, 12.9, 8.8, 0.8. HRMS (ESI-TOF) m/z: $[\mathrm{M}+\mathrm{H}]^{+}$ calculated for $\mathrm{C}_{25} \mathrm{H}_{30} \mathrm{~F}_{7} \mathrm{~N}_{2} \mathrm{O}_{2} \mathrm{Si}^{+}$551.1965, found 551.1956. 
<smiles>CCC(C)(CCc1ccccc1)C(C)=NOCC(=O)Nc1c(F)c(F)c(C(F)(F)F)c(F)c1F</smiles>

\section{(E)-2-(((3-methyl-5-phenyl-3-((trimethylsilyl)methyl)pentan-2-} ylidene)amino)oxy)-N-(2,3,5,6-tetrafluoro-4-(trifluoromethyl)phenyl)acetamide (2d)

Substrate 1d was silylated following the general silylation procedure. After purification by column chromatography (Hexane : EtOAc $=10: 1$ as eluent), 2d was obtained as a pale yellow oil $(33.6 \mathrm{mg}, 61 \%) .{ }^{1} \mathrm{H}$ NMR $\left(500 \mathrm{MHz}, \mathrm{CDCl}_{3}\right) \delta 8.11(\mathrm{br} \mathrm{s}, 1 \mathrm{H}), 7.28$ $7.23(\mathrm{t}, J=7.3 \mathrm{~Hz}, 2 \mathrm{H}), 7.18(\mathrm{t}, J=7.3 \mathrm{~Hz}, 1 \mathrm{H}), 7.12(\mathrm{~d}, J=7.2 \mathrm{~Hz}, 2 \mathrm{H}), 4.73(\mathrm{~s}, 2 \mathrm{H})$, $2.54(\mathrm{td}, J=13.0,5.1 \mathrm{~Hz}, 1 \mathrm{H}), 2.34(\mathrm{td}, J=13.0,4.6 \mathrm{~Hz}, 1 \mathrm{H}), 1.94(\mathrm{~s}, 3 \mathrm{H}), 1.92-1.82$ $(\mathrm{td}, J=13.0,5.0 \mathrm{~Hz}, 1 \mathrm{H}), 1.79-1.71(\mathrm{td}, J=13.0,4.8 \mathrm{~Hz}, 1 \mathrm{H}), 1.28(\mathrm{~s}, 3 \mathrm{H}), 0.97(\mathrm{dd}, J$ $=55.3,14.9 \mathrm{~Hz}, 2 \mathrm{H}), 0.03(\mathrm{~s}, 9 \mathrm{H}) .{ }^{13} \mathrm{C} \mathrm{NMR}\left(126 \mathrm{MHz}, \mathrm{CDCl}_{3}\right) \delta 168.6,167.5,142.1$, 128.5, 128.2 , 125.9, 72.4, 44.6, 43.6, 31.1, 28.9, 23.9, 11.5, 0.6. HRMS (ESI-TOF) m/z: $[\mathrm{M}+\mathrm{H}]^{+}$calculated for $\mathrm{C}_{25} \mathrm{H}_{30} \mathrm{~F}_{7} \mathrm{~N}_{2} \mathrm{O}_{2} \mathrm{Si}^{+}$551.1965, found 551.1963 .<smiles>CCCC(C)(CCCOc1cc(C)ccc1C)/C(C)=N/OCC(=O)Nc1c(F)c(F)c(C(F)(F)F)c(F)c1F</smiles>

(E)-2-(((6-(2,5-dimethylphenoxy)-3-methyl-3-((trimethylsilyl)methyl)hexan-2ylidene)amino)oxy)-N-(2,3,5,6-tetrafluoro-4-(trifluoromethyl)phenyl)acetamide (2e)

Substrate 1e was silylated following the general silylation procedure. After purification by column chromatography (Hexane : EtOAc $=10: 1$ as eluent), 2e was obtained as a colorless oil (43.8 mg, 72\%). ${ }^{1} \mathrm{H}$ NMR $\left(500 \mathrm{MHz}, \mathrm{CDCl}_{3}\right) \delta 8.08$ (br s, $\left.1 \mathrm{H}\right), 6.98$ (d, $J$ $=7.5 \mathrm{~Hz}, 1 \mathrm{H}), 6.65(\mathrm{~d}, J=7.5 \mathrm{~Hz}, 1 \mathrm{H}), 6.58(\mathrm{~s}, 1 \mathrm{H}), 4.72(\mathrm{~s}, 2 \mathrm{H}), 3.99-3.81(\mathrm{~m}, 2 \mathrm{H})$, $2.31(\mathrm{~d}, J=6.0 \mathrm{~Hz}, 3 \mathrm{H}), 2.13(\mathrm{~s}, 3 \mathrm{H}), 1.94(\mathrm{~s}, 3 \mathrm{H}), 1.85-1.72(\mathrm{~m}, 2 \mathrm{H}), 1.27(\mathrm{t}, J=7.1$ $\mathrm{Hz}, 2 \mathrm{H}), 1.23$ (s, 3H), $0.95(\mathrm{dd}, J=59.7,14.9 \mathrm{~Hz}, 3 \mathrm{H}), 0.03(\mathrm{~s}, 9 \mathrm{H}) .{ }^{13} \mathrm{C}$ NMR $(126$ $\left.\mathrm{MHz}, \mathrm{CDCl}_{3}\right) \delta 168.7,167.6,156.8,136.6,130.4,123.4,120.8,111.8,72.4,67.6,43.3$, $38.7,29.1,24.8,23.9,21.4,15.7,11.4,0.6$. HRMS (ESI-TOF) m/z: $[\mathrm{M}+\mathrm{H}]^{+}$calculated for $\mathrm{C}_{28} \mathrm{H}_{36} \mathrm{~F}_{7} \mathrm{~N}_{2} \mathrm{O}_{3} \mathrm{Si}^{+}$609.2383, found 609.2375. 
<smiles>CCOC(=O)C(C)(CC)/C(C)=N\OCC(=O)Nc1c(F)c(F)c(C(F)(F)F)c(F)c1[15N]C</smiles>

ethyl(E)-2-methyl-3-((2-oxo-2-((2,3,5,6-tetrafluoro-4-

(trifluoromethyl)phenyl)amino)ethoxy)imino)-2-

((trimethylsilyl)methyl)butanoate (2f)

Substrate 1f was silylated following the general silylation procedure. After purification by column chromatography (Hexane : EtOAc $=10: 1$ as eluent), $\mathbf{2 f}$ was obtained as a colorless oil (31.6 mg, 61\%). ${ }^{1} \mathrm{H}$ NMR $\left(500 \mathrm{MHz}, \mathrm{CDCl}_{3}\right) \delta 8.02$ (br s, $\left.1 \mathrm{H}\right), 4.74(\mathrm{~s}$, 2H), 4.21-4.08 (m, 2H), $1.92(\mathrm{~s}, 3 \mathrm{H}), 1.42(\mathrm{~s}, 2 \mathrm{H}), 1.23(\mathrm{t}, J=7.1 \mathrm{~Hz}, 3 \mathrm{H}), 0.03(\mathrm{~s}, 9 \mathrm{H})$. ${ }^{13} \mathrm{C} \mathrm{NMR}\left(126 \mathrm{MHz}, \mathrm{CDCl}_{3}\right) \delta 174.8,168.4,164.0,72.6,61.4,51.6,24.5,22.8,14.0$, 12.8, 0.2. HRMS (ESI-TOF) $\mathrm{m} / \mathrm{z}$ : $[\mathrm{M}+\mathrm{H}]^{+}$calculated for $\mathrm{C}_{20} \mathrm{H}_{26} \mathrm{~F}_{7} \mathrm{~N}_{2} \mathrm{O}_{4} \mathrm{Si}^{+}$519.155, found 519.1544 .<smiles>CCOC(=O)C(CSc1ccccc1)(Cc1ccccc1)C(C)=NOCC(=O)Nc1c(F)c(F)c(C(F)(F)F)c(F)c1C(C)=O</smiles>

ethyl(E)-2-benzyl-3-((2-oxo-2-((2,3,5,6-tetrafluoro-4-

(trifluoromethyl)phenyl)amino)ethoxy)imino)-2-

((trimethylsilyl)methyl)butanoate (2g)

Substrate $1 \mathrm{~g}$ was silylated following the general silylation procedure. After purification by column chromatography (Hexane : EtOAc $=10: 1$ as eluent), $\mathbf{2 g}$ was obtained as a white solid (32.6 mg, 63\%). ${ }^{1} \mathrm{H}$ NMR $\left(500 \mathrm{MHz}, \mathrm{CDCl}_{3}\right) \delta 7.57$ (br s, $\left.1 \mathrm{H}\right), 7.11$ (t, $J=$ $7.6 \mathrm{~Hz}, 2 \mathrm{H}), 7.05$ (d, $J=7.2 \mathrm{~Hz}, 2 \mathrm{H}), 6.87$ (t, $J=7.3 \mathrm{~Hz}, 1 \mathrm{H}), 4.67$ (s, 2H), 4.30-4.06 $(\mathrm{m}, 3 \mathrm{H}), 3.22-3.15(\mathrm{~m}, 1 \mathrm{H}), 1.99(\mathrm{~s}, 9 \mathrm{H}), 1.31(\mathrm{~d}, J=12.0 \mathrm{~Hz}, 1 \mathrm{H}), 1.26(\mathrm{t}, J=5.7 \mathrm{~Hz}$, $3 \mathrm{H}), 1.09(\mathrm{~d}, J=11.9 \mathrm{~Hz}, 1 \mathrm{H}), 0.05(\mathrm{~s}, 9 \mathrm{H}) .{ }^{13} \mathrm{C} \mathrm{NMR}\left(126 \mathrm{MHz}, \mathrm{CDCl}_{3}\right) \delta 173.7$, 168.5, 162.4, 136.6, 130.5, 127.8, 126.4, 72.3, 61.4, 56.4, 42.3, 22.0, 14.8, 14.0, 0.3. HRMS (ESI-TOF) m/z: $[\mathrm{M}+\mathrm{H}]^{+}$calculated for $\mathrm{C}_{26} \mathrm{H}_{30} \mathrm{~F}_{7} \mathrm{~N}_{2} \mathrm{O}_{4} \mathrm{Si}^{+}$595.1863, found 595.1857. 
<smiles>C=C(CSc1c(F)c(C(F)(F)F)c(F)c(F)c1NC(=O)CO/N=C(/C)C(=O)O)C(F)(F)F</smiles>

(E)-N-(2,3,5,6-tetrafluoro-4-(trifluoromethyl)phenyl)-2-(((3-

((trimethylsilyl)methyl)but-3-en-2-ylidene)amino)oxy)acetamide (2h)

Substrate $\mathbf{1 h}$ was silylated following the general silylation procedure. After purification by column chromatography (Hexane : EtOAc $=10: 1$ as eluent), $\mathbf{2 h}$ was obtained as a colorless oil (25.3 mg, 57\%) with an unseparatable minor $\mathrm{C}\left(\mathrm{sp}^{2}\right)-\mathrm{H}$ silylation product 2h' $(6.2 \mathrm{mg}, 14 \%)$. 2h: ${ }^{1} \mathrm{H} \mathrm{NMR}\left(500 \mathrm{MHz}, \mathrm{CDCl}_{3}\right) \delta 7.90$ (br s, 1H), $5.38(\mathrm{~s}, 1 \mathrm{H})$, $5.16(\mathrm{~s}, 1 \mathrm{H}) 4.79(\mathrm{~s}, 2 \mathrm{H}), 2.12(\mathrm{~s}, 3 \mathrm{H}), 1.89(\mathrm{~s}, 2 \mathrm{H}),-0.03(\mathrm{~s}, 9 \mathrm{H}) .{ }^{13} \mathrm{C}$ NMR $(126 \mathrm{MHz}$, $\left.\mathrm{CDCl}_{3}\right) \delta 168.2,159.7,142.5,116.2,72.8,22.2,11.2,-1.3$. HRMS (ESI-TOF) m/z: $[\mathrm{M}+\mathrm{H}]^{+}$calculated for $\mathrm{C}_{17} \mathrm{H}_{20} \mathrm{~F}_{7} \mathrm{~N}_{2} \mathrm{O}_{2} \mathrm{Si}^{+}$445.1182, found 445.1175. 2h': ${ }^{1} \mathrm{H}$ NMR (500 $\left.\mathrm{MHz}, \mathrm{CDCl}_{3}\right) \delta 8.01(\mathrm{~s}, 1 \mathrm{H}), 6.10(\mathrm{~s}, 1 \mathrm{H}), 4.78(\mathrm{~s}, 2 \mathrm{H}), 2.12$ (s, 3H), $2.01(\mathrm{~s}, 3 \mathrm{H}), 0.19$ $(\mathrm{s}, 9 \mathrm{H})$.<smiles>C/C(=N/OCC(=O)Nc1c(F)c(F)c(C(F)(F)F)c(F)c1F)C1CCC1C</smiles>

N-(2,3,5,6-tetrafluoro-4-(trifluoromethyl)phenyl)-2-((((E)-1-((1S,2S)-2(trimethylsilyl)cyclobutyl)ethylidene)amino)oxy)acetamide (2i)

Substrate 1i was silylated following the general silylation procedure. After purification by column chromatography (Hexane : EtOAc $=10: 1$ as eluent), $\mathbf{2} \mathbf{i}$ was obtained as a colorless oil $(28.4 \mathrm{mg}, 62 \%) .{ }^{1} \mathrm{H}$ NMR $\left(500 \mathrm{MHz}, \mathrm{CDCl}_{3}\right) \delta 8.11($ br s, $1 \mathrm{H}), 4.72(\mathrm{~s}$, $2 \mathrm{H}), 3.30(\mathrm{dd}, J=18.4,9.6 \mathrm{~Hz}, 1 \mathrm{H}), 2.37(\mathrm{~s}, 1 \mathrm{H}), 2.26-2.16(\mathrm{~m}, 1 \mathrm{H}), 2.04-1.96(\mathrm{~m}, 2 \mathrm{H})$, $1.88(\mathrm{~s}, 3 \mathrm{H}), 1.70(\mathrm{~m}, 1 \mathrm{H}), 0.00(\mathrm{~s}, 9 \mathrm{H}) .{ }^{13} \mathrm{C} \mathrm{NMR}\left(126 \mathrm{MHz}, \mathrm{CDCl}_{3}\right) \delta 168.6,163.5$, 72.3, 42.2, 27.8, 23.3, 19.7, 15.1, -1.8. HRMS (ESI-TOF) $\mathrm{m} / \mathrm{z}$ : $[\mathrm{M}+\mathrm{H}]^{+}$calculated for $\mathrm{C}_{18} \mathrm{H}_{22} \mathrm{~F}_{7} \mathrm{~N}_{2} \mathrm{O}_{2} \mathrm{Si}^{+} 459.1339$, found 459.1335.<smiles>CCOC(=O)C/C(=N/OCC(=O)Nc1c(F)c(F)c(C(F)(F)F)c(F)c1F)C(C)(C)CC</smiles> 


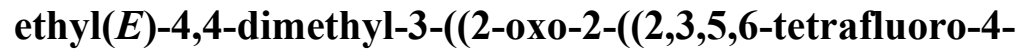

(trifluoromethyl)phenyl)amino)ethoxy)imino)-5-(trimethylsilyl)pentanoate $(2 \mathrm{j})$

Substrate $\mathbf{1} \mathbf{j}$ was silylated following the general silylation procedure. After purification by column chromatography (Hexane : EtOAc $=10: 1$ as eluent), $\mathbf{2} \mathbf{j}$ was obtained as a colorless oil $(21.2 \mathrm{mg}, 40 \%) .{ }^{1} \mathrm{H}$ NMR $\left(500 \mathrm{MHz}, \mathrm{CDCl}_{3}\right) \delta 9.28($ br s, $1 \mathrm{H}), 4.75$ (s, 2H), $4.17(\mathrm{q}, J=7.1 \mathrm{~Hz}, 2 \mathrm{H}), 3.44(\mathrm{~s}, 2 \mathrm{H}), 1.28(\mathrm{t}, J=7.1 \mathrm{~Hz}, 3 \mathrm{H}), 1.18(\mathrm{~s}, 6 \mathrm{H}), 0.86$ (s, 2H), 0.02 (s, 9H). $\left.{ }^{13} \mathrm{C} \mathrm{NMR} \mathrm{(126} \mathrm{MHz,} \mathrm{CDCl}_{3}\right) \delta 170.4,169.3,162.6,72.8,62.1$, 40.1, 31.6, 29.5, 27.7, 14.0, 0.5. HRMS (ESI-TOF) $\mathrm{m} / \mathrm{z}:[\mathrm{M}+\mathrm{H}]^{+}$calculated for $\mathrm{C}_{21} \mathrm{H}_{28} \mathrm{~F}_{7} \mathrm{~N}_{2} \mathrm{O}_{4} \mathrm{Si}^{+}$533.1706, found 533.1706.<smiles>CCC/C(=N/OCC(=O)Nc1c(F)c(F)c(C(F)(F)F)c(F)c1F)C(C)(C)CC</smiles>

(E)-2-(((2,2-dimethyl-1-(trimethylsilyl)hexan-3-ylidene)amino)oxy)-N-(2,3,5,6tetrafluoro-4-(trifluoromethyl)phenyl)acetamide (2k)

Substrate $1 \mathbf{k}$ was silylated following the general silylation procedure. After purification by column chromatography (Hexane : EtOAc $=10: 1$ as eluent), $2 \mathbf{k}$ was obtained as a colorless oil (34.6 mg, 71\%). ${ }^{1} \mathrm{H}$ NMR $\left(500 \mathrm{MHz}, \mathrm{CDCl}_{3}\right) \delta 8.13$ (br s, $\left.1 \mathrm{H}\right), 4.67$ (s, 2H), 2.36-2.22 (m, 2H), 1.64-1.59 (m, 2H), $1.19(\mathrm{~s}, 6 \mathrm{H}), 1.01(\mathrm{t}, J=7.3 \mathrm{~Hz}, 3 \mathrm{H}), 0.91$ (s, 2H), 0.01 (s, 9H). ${ }^{13} \mathrm{C} \mathrm{NMR}\left(126 \mathrm{MHz}, \mathrm{CDCl}_{3}\right) \delta 172.1,168.8,72.3,40.5,30.1,29.2$, 28.5, 20.8, 15.0, 0.6. HRMS (ESI-TOF) $\mathrm{m} / \mathrm{z}$ : $[\mathrm{M}+\mathrm{H}]^{+}$calculated for $\mathrm{C}_{20} \mathrm{H}_{28} \mathrm{~F}_{7} \mathrm{~N}_{2} \mathrm{O}_{2} \mathrm{Si}^{+}$ 489.1808 , found 489.1805 .<smiles>C[SiH2]CC(C)(C)/C(CCCCl)=N\OCC(=O)Nc1c(F)c(F)c(C(F)(F)F)c(F)c1F</smiles>

(E)-2-(((7-chloro-2,2-dimethyl-1-(trimethylsilyl)heptan-3-ylidene)amino)oxy)-N(2,3,5,6-tetrafluoro-4-(trifluoromethyl)phenyl)acetamide (2l)

Substrate 11 was silylated following the general silylation procedure. After purification by column chromatography (Hexane : EtOAc $=10: 1$ as eluent), $2 \mathbf{l}$ was obtained as a colorless oil (35.4 mg, 66\%). ${ }^{1} \mathrm{H}$ NMR $\left(500 \mathrm{MHz}, \mathrm{CDCl}_{3}\right) \delta 8.11$ (br s, $\left.1 \mathrm{H}\right), 4.68(\mathrm{~s}$, $2 \mathrm{H}), 3.59(\mathrm{t}, J=6.3 \mathrm{~Hz}, 2 \mathrm{H}), 2.34(\mathrm{dd}, J=9.8,6.5 \mathrm{~Hz}, 2 \mathrm{H}), 1.87(\mathrm{dt}, J=13.4,6.7 \mathrm{~Hz}$, $2 \mathrm{H}), 1.73(\mathrm{dt}, J=19.7,7.6 \mathrm{~Hz}, 2 \mathrm{H}), 1.21(\mathrm{~s}, 6 \mathrm{H}), 0.92(\mathrm{~s}, 2 \mathrm{H}), 0.02(\mathrm{~s}, 9 \mathrm{H}) .{ }^{13} \mathrm{C} \mathrm{NMR}$ $\left(126 \mathrm{MHz}, \mathrm{CDCl}_{3}\right) \delta 171.5,168.6,72.4,44.3,40.5,32.9,30.0,28.4,26.1,24.3,0.6$. 
HRMS (ESI-TOF) m/z: $[\mathrm{M}+\mathrm{H}]^{+}$calculated for $\mathrm{C}_{21} \mathrm{H}_{29} \mathrm{ClF}_{7} \mathrm{~N}_{2} \mathrm{O}_{2} \mathrm{Si}^{+}$537.1575, found 537.1565 .<smiles>CCC(C)(C)/C(=N\OCC(=O)Nc1c(F)c(F)c(C(F)(F)F)c(F)c1F)c1ccccc1</smiles>

\section{(E)-2-(((2,2-dimethyl-1-phenyl-3-(trimethylsilyl)propylidene)amino)oxy)-N- (2,3,5,6-tetrafluoro-4-(trifluoromethyl)phenyl)acetamide (2m)}

Substrate 1m was silylated following the general silylation procedure. After purification by column chromatography (Hexane : EtOAc $=10: 1$ as eluent), $\mathbf{2 m}$ was obtained as a white solid (44.2 mg, 80\%). $\left.{ }^{1} \mathrm{H} \mathrm{NMR} \mathrm{(500} \mathrm{MHz,} \mathrm{CDCl}_{3}\right) \delta 7.67$ (br s, $1 \mathrm{H}), 7.45(\mathrm{t}, J=7.2 \mathrm{~Hz}, 2 \mathrm{H}), 7.41(\mathrm{t}, J=7.2 \mathrm{~Hz}, 1 \mathrm{H}), 7.13-7.07(\mathrm{~m}, 2 \mathrm{H}), 4.66(\mathrm{~s}, 2 \mathrm{H})$, $1.24(\mathrm{~s}, 6 \mathrm{H}), 1.01(\mathrm{~s}, 2 \mathrm{H}), 0.09(\mathrm{~s}, 9 \mathrm{H}) .{ }^{13} \mathrm{C} \mathrm{NMR}\left(126 \mathrm{MHz}, \mathrm{CDCl}_{3}\right) \delta 170.7,167.9$, 133.6, 128.3, 127.1, 72.5, 40.5, 29.8, 28.8, 1.0. HRMS (ESI-TOF) m/z: $[\mathrm{M}+\mathrm{H}]^{+}$ calculated for $\mathrm{C}_{23} \mathrm{H}_{27} \mathrm{~F}_{7} \mathrm{~N}_{2} \mathrm{O}_{2} \mathrm{Si}^{+}$523.1652, found 523.1639 .<smiles>CCC(C)(C)/C(=N\OCC(=O)Nc1c(F)c(F)c(C(F)(F)F)c(F)c1F)c1cccc(OC)c1</smiles>

\section{(E)-2-(((1-(3-methoxyphenyl)-2,2-dimethyl-3-}

(trimethylsilyl)propylidene)amino)oxy)-N-(2,3,5,6-tetrafluoro-4-

(trifluoromethyl)phenyl)acetamide (2n)

Substrate 1n was silylated following the general silylation procedure. After purification by column chromatography (Hexane : EtOAc $=10: 1$ as eluent), $\mathbf{2 n}$ was obtained as a white solid (35.4 mg, 64\%). ${ }^{1} \mathrm{H}$ NMR $\left(500 \mathrm{MHz}, \mathrm{CDCl}_{3}\right) \delta 7.71$ (br s, 1H), $7.36(\mathrm{t}, J=$ $7.9 \mathrm{~Hz}, 1 \mathrm{H}), 6.93(\mathrm{dd}, J=8.3,2.1 \mathrm{~Hz}, 1 \mathrm{H}), 6.67(\mathrm{~d}, J=7.5 \mathrm{~Hz}, 1 \mathrm{H}), 6.63(\mathrm{~s}, 1 \mathrm{H}), 4.66$ $(\mathrm{s}, 2 \mathrm{H}), 3.82(\mathrm{~s}, 3 \mathrm{H}), 1.24(\mathrm{~s}, 6 \mathrm{H}), 1.02(\mathrm{~s}, 2 \mathrm{H}), 0.09(\mathrm{~s}, 9 \mathrm{H}) \cdot{ }^{13} \mathrm{C}$ NMR $(126 \mathrm{MHz}$, $\left.\mathrm{CDCl}_{3}\right) \delta 170.5,168.0,159.5,134.9,129.5,119.3,113.7,112.9,72.6,55.2,40.4,29.8$, 28.9, 1.0. HRMS (ESI-TOF) $\mathrm{m} / \mathrm{z}$ : $[\mathrm{M}+\mathrm{H}]^{+}$calculated for $\mathrm{C}_{24} \mathrm{H}_{28} \mathrm{~F}_{7} \mathrm{~N}_{2} \mathrm{O}_{3} \mathrm{Si}^{+}$553.1757, found 553.1746 . 
<smiles>COC(=O)CO/N=C(/c1ccc(C)cc1)C(C)(C)CSC(=O)Nc1c(F)c(F)c(C(F)(F)F)c(F)c1F</smiles>

\section{(E)-2-(((2,2-dimethyl-1-(p-tolyl)-3-(trimethylsilyl)propylidene)amino)oxy)-N- (2,3,5,6-tetrafluoro-4-(trifluoromethyl)phenyl)acetamide (2o)}

Substrate 10 was silylated following the general silylation procedure. After purification by column chromatography (Hexane : EtOAc $=10: 1$ as eluent), 2o was obtained as a white solid (38.6 mg, 72\%). ${ }^{1} \mathrm{H}$ NMR (500 MHz, $\left.\mathrm{CDCl}_{3}\right) \delta 7.72$ (br s, 1H), 7.25 (d, J = $7.9 \mathrm{~Hz}, 2 \mathrm{H}), 6.99$ (d, J = 7.9 Hz, 2H), 4.66 (s, 2H), 2.40 (s, 3H), 1.23 (s, 6H), 1.00 (s, $2 \mathrm{H}), 0.08(\mathrm{~s}, 9 \mathrm{H}) .{ }^{13} \mathrm{C} \mathrm{NMR}\left(126 \mathrm{MHz}, \mathrm{CDCl}_{3}\right) \delta 170.9,168.1,138.2,130.5,129.0$, 127.0, 72.5, 40.5, 29.8, 28.8, 21.3, 1.0. HRMS (ESI-TOF) $\mathrm{m} / \mathrm{z}$ : $[\mathrm{M}+\mathrm{H}]^{+}$calculated for $\mathrm{C}_{24} \mathrm{H}_{28} \mathrm{~F}_{7} \mathrm{~N}_{2} \mathrm{O}_{2} \mathrm{Si}^{+}$537.1808, found 537.1800.<smiles>CCC(C)(C)/C(=N\OCC(=O)Nc1c(F)c(F)c(C(F)(F)F)c(F)c1F)c1ccc(C(C)(C)C)cc1</smiles>

(E)-2-(((1-(4-(tert-butyl)phenyl)-2,2-dimethyl-3-

(trimethylsilyl)propylidene)amino)oxy)-N-(2,3,5,6-tetrafluoro-4-

(trifluoromethyl)phenyl)acetamide (2p)

Substrate 1p was silylated following the general silylation procedure. After purification by column chromatography (Hexane : EtOAc $=10: 1$ as eluent), $\mathbf{2 p}$ was obtained as a white solid (42.8 mg, 74\%). ${ }^{1} \mathrm{H}$ NMR (500 MHz, $\left.\mathrm{CDCl}_{3}\right) \delta 7.71$ (br s, 1H), 7.44 (d, $J=$ $8.3 \mathrm{~Hz}, 2 \mathrm{H}), 7.02$ (d, $J=8.3 \mathrm{~Hz}, 2 \mathrm{H}), 4.66$ (s, 2H), 1.34 (s, 9H), 1.23 (s, 6H), 1.02 (s, 2H), 0.09 (s, 9H). ${ }^{13} \mathrm{C}$ NMR $\left(126 \mathrm{MHz}, \mathrm{CDCl}_{3}\right) \delta 170.9,168.0,151.2,130.5,126.7$, 125.2, 72.5, 40.5, 34.7, 31.2, 29.7, 28.9, 1.0. HRMS (ESI-TOF) $\mathrm{m} / \mathrm{z}$ : $[\mathrm{M}+\mathrm{H}]^{+}$calculated for $\mathrm{C}_{27} \mathrm{H}_{34} \mathrm{~F}_{7} \mathrm{~N}_{2} \mathrm{O}_{2} \mathrm{Si}^{+}$579.2278, found 579.2270. 
<smiles>CCC(C)(C)/C(=N\OCC(=O)Nc1c(F)c(F)c(C(F)(F)F)c(F)c1F)c1ccc(F)cc1</smiles>

\section{(E)-2-(((1-(4-fluorophenyl)-2,2-dimethyl-3-}

(trimethylsilyl)propylidene)amino)oxy)-N-(2,3,5,6-tetrafluoro-4(trifluoromethyl)phenyl)acetamide (2q)

Substrate 1q was silylated following the general silylation procedure. After purification by column chromatography (Hexane : EtOAc $=10: 1$ as eluent), $2 \mathbf{q}$ was obtained as a white solid (33.0 mg, 61\%). ${ }^{1} \mathrm{H}$ NMR $\left(500 \mathrm{MHz}, \mathrm{CDCl}_{3}\right) \delta 7.65$ (br s, $\left.1 \mathrm{H}\right), 7.15(\mathrm{t}, J=$ $8.5 \mathrm{~Hz}, 2 \mathrm{H}), 7.12-7.05(\mathrm{~m}, 2 \mathrm{H}), 4.65$ (s, 2H), 1.23 (s, 6H), 0.98 (s, 2H), 0.08 (s, 9H). ${ }^{13} \mathrm{C} \mathrm{NMR}\left(126 \mathrm{MHz}, \mathrm{CDCl}_{3}\right) \delta 170.0,167.7,163.5,161.5,129.3,129.3,129.0,129.0$, 115.6, 115.5, 72.6, 40.5, 29.8, 28.7, 0.9. HRMS (ESI-TOF) $\mathrm{m} / \mathrm{z}:[\mathrm{M}+\mathrm{H}]^{+}$calculated for $\mathrm{C}_{23} \mathrm{H}_{25} \mathrm{~F}_{8} \mathrm{~N}_{2} \mathrm{O}_{2} \mathrm{Si}^{+}$541.1557, found 541.1543.<smiles>C[SiH2]CC(C)(C)C(=NOCC(=O)Nc1c(F)c(F)c(C(F)(F)F)c(F)c1F)c1ccc(Cl)cc1</smiles>

\section{(E)-2-(((1-(4-chlorophenyl)-2,2-dimethyl-3-}

(trimethylsilyl)propylidene)amino)oxy)-N-(2,3,5,6-tetrafluoro-4-

(trifluoromethyl)phenyl)acetamide (2r)

Substrate 1r was silylated following the general silylation procedure. After purification by column chromatography (Hexane : EtOAc $=10: 1$ as eluent), $2 \mathbf{r}$ was obtained as a white solid (36.7 mg, 66\%). ${ }^{1} \mathrm{H}$ NMR $\left(500 \mathrm{MHz}, \mathrm{CDCl}_{3}\right) \delta 7.64$ (br s, 1H), $7.43(\mathrm{~d}, J=$ $8.4 \mathrm{~Hz}, 2 \mathrm{H}), 7.05(\mathrm{~d}, J=8.4 \mathrm{~Hz}, 2 \mathrm{H}), 4.65(\mathrm{~s}, 2 \mathrm{H}), 1.23(\mathrm{~s}, 6 \mathrm{H}), 0.98(\mathrm{~s}, 2 \mathrm{H}), 0.08$ (s, 9H). ${ }^{13} \mathrm{C} \mathrm{NMR}\left(126 \mathrm{MHz}, \mathrm{CDCl}_{3}\right) \delta 169.9,167.7,134.5,131.8,128.7,128.6,72.6,40.5$, 29.8, 28.7, 1.0. HRMS (ESI-TOF) $\mathrm{m} / \mathrm{z}$ : $[\mathrm{M}+\mathrm{H}]^{+}$calculated for $\mathrm{C}_{23} \mathrm{H}_{25} \mathrm{ClF}_{7} \mathrm{~N}_{2} \mathrm{O}_{2} \mathrm{Si}^{+}$ 557.1262 , found 557.1256 . 
<smiles>CCC(C)(C)/C(=N\OCC(=O)Nc1c(F)c(F)c(C(F)(F)F)c(F)c1F)c1ccc(OC)cc1</smiles>

\section{(E)-2-(((1-(4-methoxyphenyl)-2,2-dimethyl-3-}

\section{(trimethylsilyl)propylidene)amino)oxy)-N-(2,3,5,6-tetrafluoro-4-}

(trifluoromethyl)phenyl)acetamide (2s)

Substrate 1s was silylated following the general silylation procedure. After purification by column chromatography (Hexane : EtOAc $=10: 1$ as eluent), $\mathbf{2} \mathbf{s}$ was obtained as a white solid (36.4 mg, 66\%). ${ }^{1} \mathrm{H}$ NMR (500 MHz, $\left.\mathrm{CDCl}_{3}\right) \delta 7.72$ (br s, $\left.1 \mathrm{H}\right), 7.03(\mathrm{~d}, J=$ $8.6 \mathrm{~Hz}, 2 \mathrm{H}), 6.96(\mathrm{~d}, J=8.7 \mathrm{~Hz}, 2 \mathrm{H}), 4.66(\mathrm{~s}, 2 \mathrm{H}), 3.84(\mathrm{~s}, 3 \mathrm{H}), 1.22(\mathrm{~s}, 6 \mathrm{H}), 0.98$ (s, 2H), $0.07(\mathrm{~s}, 9 \mathrm{H}) .{ }^{13} \mathrm{C}$ NMR $\left(126 \mathrm{MHz}, \mathrm{CDCl}_{3}\right) \delta 170.7,168.0,159.4,128.4,125.5$, $113.8,72.5,55.3,40.6,29.8,28.8,1.0$. HRMS (ESI-TOF) $\mathrm{m} / \mathrm{z}$ : $[\mathrm{M}+\mathrm{H}]^{+}$calculated for $\mathrm{C}_{24} \mathrm{H}_{28} \mathrm{~F}_{7} \mathrm{~N}_{2} \mathrm{O}_{3} \mathrm{Si}^{+}$553.1757, found 553.1746.<smiles>CCC(C)(CC)C(=NOCC(=O)Nc1c(F)c(F)c(C(F)(F)F)c(F)c1F)c1cccs1</smiles>

(Z)-2-(((2,2-dimethyl-1-(thiophen-2-yl)-3-(trimethylsilyl)propylidene)amino)oxy)N-(2,3,5,6-tetrafluoro-4-(trifluoromethyl)phenyl)acetamide (2t)

Substrate 1t was silylated following the general silylation procedure. After purification by column chromatography (Hexane : EtOAc $=10: 1$ as eluent), 2t was obtained as a pule yellow oil $(34.3 \mathrm{mg}, 65 \%) .{ }^{1} \mathrm{H} \mathrm{NMR}\left(500 \mathrm{MHz}, \mathrm{CDCl}_{3}\right) \delta 7.82$ (br s, $\left.1 \mathrm{H}\right), 7.49$ $(\mathrm{dd}, J=5.0,1.0 \mathrm{~Hz}, 1 \mathrm{H}), 7.14(\mathrm{dd}, J=5.0,3.7 \mathrm{~Hz}, 1 \mathrm{H}), 7.11(\mathrm{dd}, J=3.6,1.0 \mathrm{~Hz}, 1 \mathrm{H})$, $4.77(\mathrm{~s}, 2 \mathrm{H}), 1.32(\mathrm{~s}, 6 \mathrm{H}), 1.09(\mathrm{~s}, 2 \mathrm{H}), 0.05(\mathrm{~s}, 9 \mathrm{H}) .{ }^{13} \mathrm{C} \mathrm{NMR}\left(126 \mathrm{MHz}, \mathrm{CDCl}_{3}\right) \delta$ 167.7, 163.7, 130.7, 128.1, 127.2, 126.7, 72.8, 41.1, 30.2, 29.4, 0.8. HRMS (ESI-TOF) $\mathrm{m} / \mathrm{z}:[\mathrm{M}+\mathrm{H}]^{+}$calculated for $\mathrm{C}_{21} \mathrm{H}_{24} \mathrm{~F}_{7} \mathrm{~N}_{2} \mathrm{O}_{2} \mathrm{SSi}^{+}$529.1216, found 529.1217.<smiles>CCC(C)(C)C(C=Cc1ccccc1)=NOCC(=O)Nc1c(F)c(F)c(C(F)(F)F)c(F)c1F</smiles> 
2-((((1E,3E)-4,4-dimethyl-1-phenyl-5-(trimethylsilyl)pent-1-en-3ylidene)amino)oxy)-N-(2,3,5,6-tetrafluoro-4-(trifluoromethyl)phenyl)acetamide (2u)

Substrate 1u was silylated following the general silylation procedure. After purification by column chromatography (Hexane : EtOAc $=10: 1$ as eluent), $\mathbf{2 u}$ was obtained as a white solid (39.0 mg, 71\%). ${ }^{1} \mathrm{H}$ NMR (500 MHz, $\left.\mathrm{CDCl}_{3}\right) \delta 8.08$ (br s, $\left.1 \mathrm{H}\right), 7.50(\mathrm{~d}, J=$ $7.3 \mathrm{~Hz}, 2 \mathrm{H}), 7.47(\mathrm{~d}, J=17.0 \mathrm{~Hz}, 1 \mathrm{H}), 7.40(\mathrm{t}, J=7.3 \mathrm{~Hz}, 2 \mathrm{H}), 7.36(\mathrm{~d}, J=7.1 \mathrm{~Hz}$, $1 \mathrm{H}), 6.65(\mathrm{~d}, J=16.8 \mathrm{~Hz}, 1 \mathrm{H}), 4.78(\mathrm{~s}, 2 \mathrm{H}), 1.06(\mathrm{~s}, 2 \mathrm{H}), 1.31(\mathrm{~s}, 6 \mathrm{H}), 0.04(\mathrm{~s}, 9 \mathrm{H}) .{ }^{13} \mathrm{C}$ NMR $\left(126 \mathrm{MHz}, \mathrm{CDCl}_{3}\right) \delta 168.4,165.2,140.5,136.3,129.3,128.9,127.0,116.6,72.8$, 40.7, 31.1, 29.8, 0.6. HRMS (ESI-TOF) $\mathrm{m} / \mathrm{z}$ : $[\mathrm{M}+\mathrm{H}]^{+}$calculated for $\mathrm{C}_{25} \mathrm{H}_{28} \mathrm{~F}_{7} \mathrm{~N}_{2} \mathrm{O}_{2} \mathrm{Si}^{+}$ 549.1808 , found 549.1810 .<smiles>CCC(C)(C)C(/C=C/c1ccccc1[N+](=O)[O-])=N\OCC(=O)Nc1c(F)c(F)c(C(F)(F)F)c(F)c1F</smiles>

2-((((1E,3E)-4,4-dimethyl-1-(2-nitrophenyl)-5-(trimethylsilyl)pent-1-en-3ylidene)amino)oxy)-N-(2,3,5,6-tetrafluoro-4-(trifluoromethyl)phenyl)acetamide $(2 \mathrm{v})$

Substrate $1 \mathbf{v}$ was silylated following the general silylation procedure. After purification by column chromatography (Hexane : EtOAc $=6: 1$ as eluent), $2 \mathbf{v}$ was obtained as a pale yellow oil (30.4 mg, 55\%). ${ }^{1} \mathrm{H}$ NMR $\left(500 \mathrm{MHz}, \mathrm{CDCl}_{3}\right) \delta 8.08$ (br s, 1H), 8.05 $(\mathrm{dd}, J=8.2,0.9 \mathrm{~Hz}, 1 \mathrm{H}), 7.79(\mathrm{~d}, J=16.7 \mathrm{~Hz}, 1 \mathrm{H}), 7.69$ (t, $J=7.5 \mathrm{~Hz}, 1 \mathrm{H}), 7.63(\mathrm{~d}, J$ $=7.7 \mathrm{~Hz}, 1 \mathrm{H}), 7.56-7.50(\mathrm{~m}, 1 \mathrm{H}), 6.56(\mathrm{t}, J=12.1 \mathrm{~Hz}, 1 \mathrm{H}), 4.82(\mathrm{~s}, 2 \mathrm{H}), 1.32(\mathrm{~s}, 6 \mathrm{H})$, 1.07 (s, 2H), 0.05 (s, 9H). ${ }^{13} \mathrm{C}$ NMR $\left(126 \mathrm{MHz}, \mathrm{CDCl}_{3}\right) \delta 168.0,164.4,147.9,135.4$, 133.8, 132.9, 129.5, 128.9, 125.0, 121.8, 73.2, 40.7, 30.9, 29.6, 28.7, 0.6. HRMS (ESITOF) m/z: $[\mathrm{M}+\mathrm{H}]^{+}$calculated for $\mathrm{C}_{25} \mathrm{H}_{27} \mathrm{~F}_{7} \mathrm{~N}_{3} \mathrm{O}_{4} \mathrm{Si}^{+}$594.1659, found 594.1646.<smiles>CCC(C)(C)C(/C=C/c1cccc(C#N)c1)=N\OCC(=O)Nc1c(F)c(F)c(C(F)(F)F)c(F)c1F</smiles>

2-((((1E,3E)-1-(3-cyanophenyl)-4,4-dimethyl-5-(trimethylsilyl)pent-1-en-3ylidene)amino)oxy)-N-(2,3,5,6-tetrafluoro-4-(trifluoromethyl)phenyl)acetamide (2w)

Substrate 1w was silylated following the general silylation procedure. After purification 
by column chromatography (Hexane : EtOAc $=6: 1$ as eluent), $\mathbf{2} \mathbf{w}$ was obtained as a white solid (36.7 mg, 64\%). ${ }^{1} \mathrm{H}$ NMR $\left(500 \mathrm{MHz}, \mathrm{CDCl}_{3}\right) \delta 7.98($ br s, $1 \mathrm{H}), 7.77(\mathrm{~s}, 1 \mathrm{H})$, $7.70(\mathrm{~d}, J=7.9 \mathrm{~Hz}, 1 \mathrm{H}), 7.63(\mathrm{~d}, J=7.7 \mathrm{~Hz}, 1 \mathrm{H}), 7.52(\mathrm{t}, J=7.8 \mathrm{~Hz}, 1 \mathrm{H}), 7.41(\mathrm{~d}, J=$ $16.8 \mathrm{~Hz}, 1 \mathrm{H}), 6.66(\mathrm{~d}, J=16.8 \mathrm{~Hz}, 1 \mathrm{H}), 4.79(\mathrm{~s}, 2 \mathrm{H}), 1.31(\mathrm{~s}, 6 \mathrm{H}), 1.04(\mathrm{~s}, 2 \mathrm{H}), 0.04$ (s, 9H). ${ }^{13} \mathrm{C}$ NMR $\left(126 \mathrm{MHz}, \mathrm{CDCl}_{3}\right) \delta 168.1,164.6,137.8,137.5,132.2,131.1,130.3$, $129.8,119.1,118.4,113.3,72.9,40.8,31.0,29.6,0.6$. HRMS (ESI-TOF) $\mathrm{m} / \mathrm{z}:[\mathrm{M}+\mathrm{H}]^{+}$ calculated for $\mathrm{C}_{26} \mathrm{H}_{27} \mathrm{~F}_{7} \mathrm{~N}_{3} \mathrm{O}_{2} \mathrm{Si}^{+}$574.1761, found 574.1752 .<smiles>CCC(C)(C)C(/C=C/c1ccc([N+](=O)[O-])cc1)=N\OCC(=O)Nc1c(F)c(F)c(C(F)(F)F)c(F)c1F</smiles>

\section{2-((((1E,3E)-4,4-dimethyl-1-(4-nitrophenyl)-5-(trimethylsilyl)pent-1-en-3-} ylidene)amino)oxy)-N-(2,3,5,6-tetrafluoro-4-(trifluoromethyl)phenyl)acetamide $(2 \mathbf{x})$

Substrate 1x was silylated following the general silylation procedure. After purification by column chromatography (Hexane : EtOAc $=6: 1$ as eluent), $\mathbf{2 x}$ was obtained as a pale yellow oil (40.9 mg, 69\%). ${ }^{1} \mathrm{H}$ NMR $\left(500 \mathrm{MHz}, \mathrm{CDCl}_{3}\right) \delta 8.29-8.20(\mathrm{~m}, 2 \mathrm{H}), 7.98$ (br s, 1H), $7.63(\mathrm{~d}, J=8.7 \mathrm{~Hz}, 2 \mathrm{H}), 7.47(\mathrm{~d}, J=16.8 \mathrm{~Hz}, 1 \mathrm{H}), 6.75(\mathrm{~d}, J=16.8 \mathrm{~Hz}, 1 \mathrm{H})$, $4.80(\mathrm{~s}, 2 \mathrm{H}), 1.32$ (s, 6H), 1.05 (s, 2H), $0.04(\mathrm{~s}, 9 \mathrm{H}) .{ }^{13} \mathrm{C} \mathrm{NMR}\left(126 \mathrm{MHz}, \mathrm{CDCl}_{3}\right) \delta$ 168.0, 164.5, 147.9, 142.5, 137.8, 127.6, 124.2, 120.8, 73.0, 40.8, 31.0, 29.6, 0.6. HRMS (ESI-TOF) m/z: $[\mathrm{M}+\mathrm{H}]^{+}$calculated for $\mathrm{C}_{25} \mathrm{H}_{27} \mathrm{~F}_{7} \mathrm{~N}_{3} \mathrm{O}_{4} \mathrm{Si}^{+}$594.1659, found 594.1647.<smiles>CCC(C)(C)C(/C=C/c1ccc(F)cc1)=N\OCC(=O)Nc1c(F)c(F)c(C(F)(F)F)c(F)c1F</smiles>

2-((((1E,3E)-1-(4-fluorophenyl)-4,4-dimethyl-5-(trimethylsilyl)pent-1-en-3ylidene)amino)oxy)-N-(2,3,5,6-tetrafluoro-4-(trifluoromethyl)phenyl)acetamide (2y)

Substrate 1y was silylated following the general silylation procedure. After purification by column chromatography (Hexane : EtOAc $=10: 1$ as eluent), $\mathbf{2 y}$ was obtained as a white solid (39.7 mg, 70\%). ${ }^{1} \mathrm{H}$ NMR $\left(500 \mathrm{MHz}, \mathrm{CDCl}_{3}\right) \delta 8.07$ (br s, 1H), 7.50-7.43 (m, 3H), 7.09 (t, $J=8.6 \mathrm{~Hz}, 2 \mathrm{H}), 6.55(\mathrm{~d}, J=16.8 \mathrm{~Hz}, 1 \mathrm{H}), 4.78(\mathrm{~s}, 2 \mathrm{H}), 1.31(\mathrm{~s}, 6 \mathrm{H})$, 1.05 (s, 2H), 0.03 (s, 9H). ${ }^{13} \mathrm{C}$ NMR (126 MHz, $\left.\mathrm{CDCl}_{3}\right) \delta 168.4,164.9,164.3,162.3$, 
139.4, 132.5, 128.7, 128.7, 116.3, 116.1, 115.9, 72.9, 40.7, 31.1, 29.8, 0.6. HRMS (ESITOF) $\mathrm{m} / \mathrm{z}$ : $[\mathrm{M}+\mathrm{H}]^{+}$calculated for $\mathrm{C}_{25} \mathrm{H}_{27} \mathrm{~F}_{8} \mathrm{~N}_{2} \mathrm{O}_{2} \mathrm{Si}^{+}$567.1714, found 567.1701.

\section{The Removal of Auxiliary}

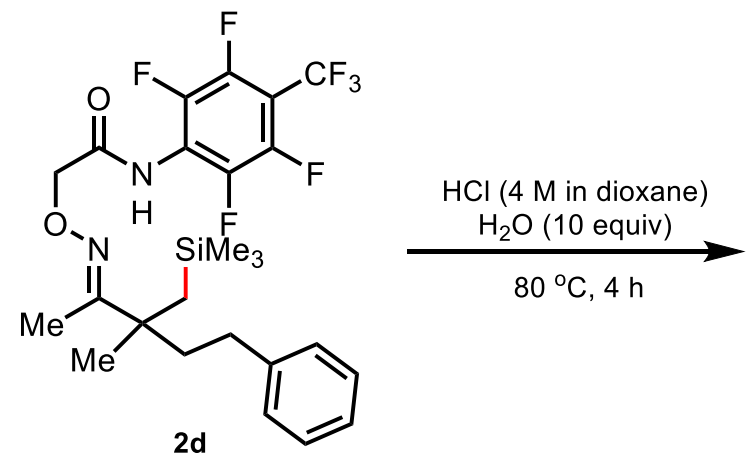

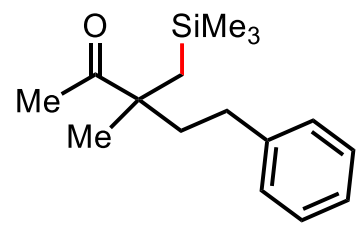

3d, $73 \%$

The removal of auxiliary: $2 \mathbf{d}(0.1 \mathrm{mmol}, 45.0 \mathrm{mg})$ was weighed into a reaction vial $\left(10 \mathrm{~mL}\right.$ ) with a magnetic stir bar under air. $9.0 \mu \mathrm{L}$ of $\mathrm{H}_{2} \mathrm{O}$ (10 equiv) and $0.5 \mathrm{~mL}$ of $\mathrm{HCl}$ (4 $\mathrm{M}$ in dioxane) were added, and the vial was sealed with a cap. The reaction mixture was stirred at $80^{\circ} \mathrm{C}$ in an oil bath for 4 hours. Upon completion, the reaction mixture was cooled to room temperature and the solvent was removed under vacuum. The resulting mixture was purified by preparative column chromatography by using Hexane/EtOAc (100:1) as the eluent to give $19.2 \mathrm{mg}$ of $\mathbf{3 d}$ (73\% yield).<smiles>CCC(C)(CCc1ccccc1)C(C)=O</smiles>

\section{3-methyl-5-phenyl-3-((trimethylsilyl)methyl)pentan-2-one (3d)}

Colorless oil (19.2 mg, 73\%). ${ }^{1} \mathrm{H}$ NMR (500 MHz, $\left.\mathrm{CDCl}_{3}\right) \delta$ 7.31-7.25 (m, 2H), 7.19 $(\mathrm{d}, J=7.4 \mathrm{~Hz}, 1 \mathrm{H}), 7.15(\mathrm{~d}, J=7.2 \mathrm{~Hz}, 2 \mathrm{H}), 2.51(\mathrm{td}, J=13.1,5.0 \mathrm{~Hz}, 1 \mathrm{H}), 2.39(\mathrm{td}, J$ $=13.1,4.7 \mathrm{~Hz}, 1 \mathrm{H}), 2.16(\mathrm{~s}, 3 \mathrm{H}), 1.92(\mathrm{td}, J=13.1,4.7 \mathrm{~Hz}, 1 \mathrm{H}), 1.79(\mathrm{td}, J=13.2,5.1$ $\mathrm{Hz}, 1 \mathrm{H}), 1.24$ (s, 3H), 1.08 (d, $J=14.7 \mathrm{~Hz}, 1 \mathrm{H}), 0.88$ (d, $J=14.7 \mathrm{~Hz}, 1 \mathrm{H}), 0.04(\mathrm{~s}, 9 \mathrm{H})$. ${ }^{13} \mathrm{C} \mathrm{NMR}\left(126 \mathrm{MHz}, \mathrm{CDCl}_{3}\right) \delta 213.5,142.2,128.5,128.3,125.9,50.6,43.8,31.2,27.5$, 25.1, 23.4, 0.6. HRMS (EI) $\mathrm{m} / \mathrm{z}$ : $\left[\mathrm{M}-\mathrm{CH}_{3}\right]^{+}$calculated for $\mathrm{C}_{15} \mathrm{H}_{23} \mathrm{OSi}^{+}$247.1518, Found: 247.1513 . 


\section{Gram-scale reaction}<smiles>CCC(C)(C)C(C)=NOCC(=O)NC(F)(F)F</smiles>

$1 \mathrm{a}, 4.0 \mathrm{mmol}$
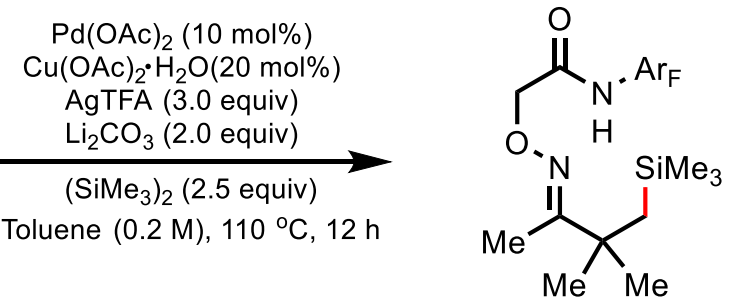

2a, $67 \%$

Substrate $1 \mathrm{a}(4.0 \mathrm{mmol}), \mathrm{Pd}(\mathrm{OAc})_{2}(0.4 \mathrm{mmol}, 88.0 \mathrm{mg}), \mathrm{Cu}(\mathrm{OAc})_{2} \cdot \mathrm{H}_{2} \mathrm{O}(0.8 \mathrm{mmol}$, $160 \mathrm{mg}), \operatorname{AgTFA}(12 \mathrm{mmol}, 2640 \mathrm{mg}), \mathrm{Li}_{2} \mathrm{CO}_{3}(8.0 \mathrm{mmol}, 592 \mathrm{mg}),\left(\mathrm{SiMe}_{3}\right)_{2}(10 \mathrm{mmol}$, $2920 \mathrm{mg})$ were weighed into a reaction vial $(100 \mathrm{~mL})$ with a magnetic stir bar under air. Toluene $(20 \mathrm{~mL})$ was added, and the vial was sealed with a cap. The reaction mixture was stirred at $110{ }^{\circ} \mathrm{C}$ in an oil bath for 12 hours. Upon completion, the reaction mixture was cooled to room temperature and diluted with EtOAc. Then the reaction mixture was filtered through a plug of celite and the solvent was removed under vacuum. The resulting mixture was purified by column chromatography (Hexane : EtOAc $=10: 1$ as eluent) to afford $\mathbf{2 a}(1.23 \mathrm{~g}, 67 \%)$.

\section{The Late-Stage $\beta$-C $\left(\mathbf{s p}^{3}\right)-H$ Silylation of Santonin}

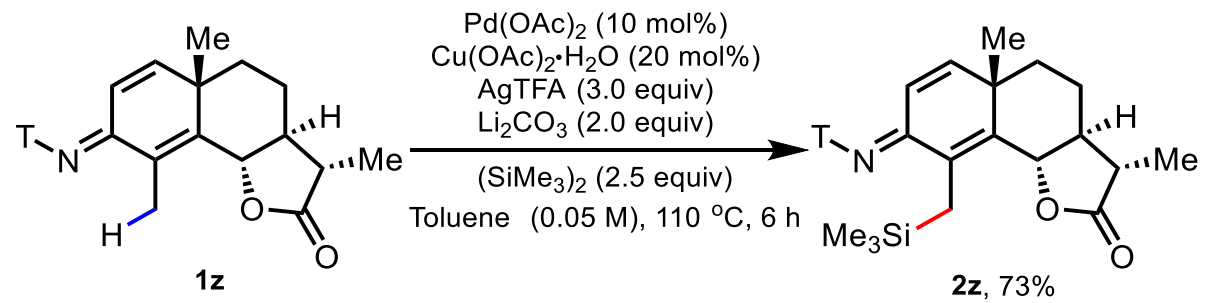

General Procedure for the Late-Stage $\boldsymbol{\beta}$-C $\left(\mathrm{sp}^{3}\right)-H$ Silylation of Santonin:

Substrate $1 z$ (0.10 mmol, $53.4 \mathrm{mg}), \mathrm{Pd}(\mathrm{OAc})_{2}(0.01 \mathrm{mmol}, 2.2 \mathrm{mg}), \mathrm{Cu}(\mathrm{OAc})_{2} \bullet \mathrm{H}_{2} \mathrm{O}$ (0.02 mmol, $4.0 \mathrm{mg}$ ), AgTFA (0.30 mmol, $66.0 \mathrm{mg}), \mathrm{Li}_{2} \mathrm{CO}_{3}(0.20 \mathrm{mmol}, 14.8 \mathrm{mg}$ ), $\left(\mathrm{SiMe}_{3}\right)_{2}(0.25 \mathrm{mmol}, 73.0 \mathrm{mg})$ were weighed into a reaction vial $(10 \mathrm{~mL})$ with a magnetic stir bar under air. Toluene $(2.0 \mathrm{~mL})$ was added, and the vial was sealed with a cap. The reaction mixture was stirred at $110^{\circ} \mathrm{C}$ in an oil bath for 6 hours. Upon completion, the reaction mixture was cooled to room temperature and diluted with EtOAc. Then the reaction mixture was filtered through a plug of celite and the solvent was removed under vacuum. T The resulting mixture was purified by column chromatography (Hexane : EtOAc $=10: 1$ as eluent) to afford to give $44.2 \mathrm{mg}$ of $\mathbf{2 z}(73 \%$ yield). 
<smiles>C[C@H]1C(=O)O[C@H]2C3=C(C[As])/C(=N/OCC(=O)Nc4c(F)c(F)c(C(F)(F)F)c(F)c4F)C=C[C@]3(C)CC[C@@H]12</smiles>

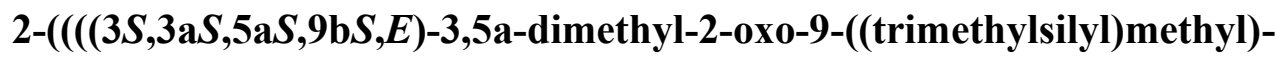
2,3,3a,5,5a,9b-hexahydronaphtho[1,2-b]furan-8(4H)-ylidene)amino)oxy)-N$\mathbf{( 2 , 3 , 5 , 6 - t e t r a f l u o r o - 4 - ( t r i f l u o r o m e t h y l ) p h e n y l ) a c e t a m i d e ~ ( 2 z ) ~}$

pale yellow oil (44.2 mg, 73\%). ${ }^{1} \mathrm{H} \mathrm{NMR}\left(500 \mathrm{MHz}, \mathrm{CDCl}_{3}\right) \delta 8.01$ (br s, $\left.1 \mathrm{H}\right), 6.89(\mathrm{~d}$, $J=10.1 \mathrm{~Hz}, 1 \mathrm{H}), 6.19$ (d, $J=10.1 \mathrm{~Hz}, 1 \mathrm{H}), 4.79$ (s, 2H), $4.76(\mathrm{~d}, J=11.3 \mathrm{~Hz}, 1 \mathrm{H})$, $2.34(\mathrm{~d}, J=7.0 \mathrm{~Hz}, 3 \mathrm{H}), 2.00(\mathrm{~d}, J=14.1 \mathrm{~Hz}, 1 \mathrm{H}), 1.85-1.76(\mathrm{~m}, 2 \mathrm{H}), 1.71-1.64(\mathrm{~m}$, $1 \mathrm{H}), 1.49(\mathrm{dd}, J=12.3,3.4 \mathrm{~Hz}, 1 \mathrm{H}), 1.28(\mathrm{~s}, 3 \mathrm{H}), 1.25(\mathrm{~s}, 2 \mathrm{H}),-0.01(\mathrm{~s}, 9 \mathrm{H}) .{ }^{13} \mathrm{C} \mathrm{NMR}$ $\left(126 \mathrm{MHz}, \mathrm{CDCl}_{3}\right) \delta 177.6,168.3,152.7,148.1,138.1,125.0,112.1,82.1,72.9,53.5$, 41.2, 41.0, 38.2, 25.9, 23.7, 15.2, 12.5, -0.4. HRMS (ESI-TOF) $\mathrm{m} / \mathrm{z}:[\mathrm{M}+\mathrm{H}]^{+}$calculated for $\mathrm{C}_{27} \mathrm{H}_{29} \mathrm{~F}_{7} \mathrm{~N}_{2} \mathrm{O}_{4} \mathrm{Si} 607.1863$, found 607.1856. 


\section{References}

(1) Peter, M.; Gleiter, R.; Rominger, F.; Oeser, T. Eur. J. Org. Chem. 2004, 3212.

(2) Yang, Q. L.; Li, Y. Q.; Ma, C.; Fang, P.; Zhang, X. J.; Mei, T. S. J. Am. Chem. Soc. 2017, 139, 3293-3298.

(3) Ushijima, S.; Dohi, S.; Moriyama, K.; Togo, H. Tetrahedron. 2012, 68, 1436.

(4) Mandal, R.; Emayavaramban, B.; Sundararaju, B. Org. Lett. 2018, 20, 2835-2838

(5) Li, X.; Li, L.; Tang, Y.; Zhong, L.; Cun, L.; Zhu, J.; Liao, J.; Deng, J. J. Org. Chem. 2010, 75, 2981.

(6) Chambers, R. D.; Martin, P. A.; Sandford, G.; Williams, D. L. H. J. Fluorine Chem. 2008, 129,998 . 


\section{${ }^{1} \mathrm{H}$ and ${ }^{13} \mathrm{C}$ NMR Spectra}

${ }^{1} \mathrm{H}$ NMR (500 MHz, $\mathrm{CDCl}_{3}$ ) spectra for $1 \mathrm{a}$<smiles>CC(=NOCC(=O)Nc1c(F)c(F)c(C(F)(F)F)c(F)c1F)C(C)(C)C</smiles>

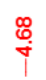

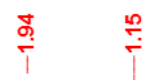

1a

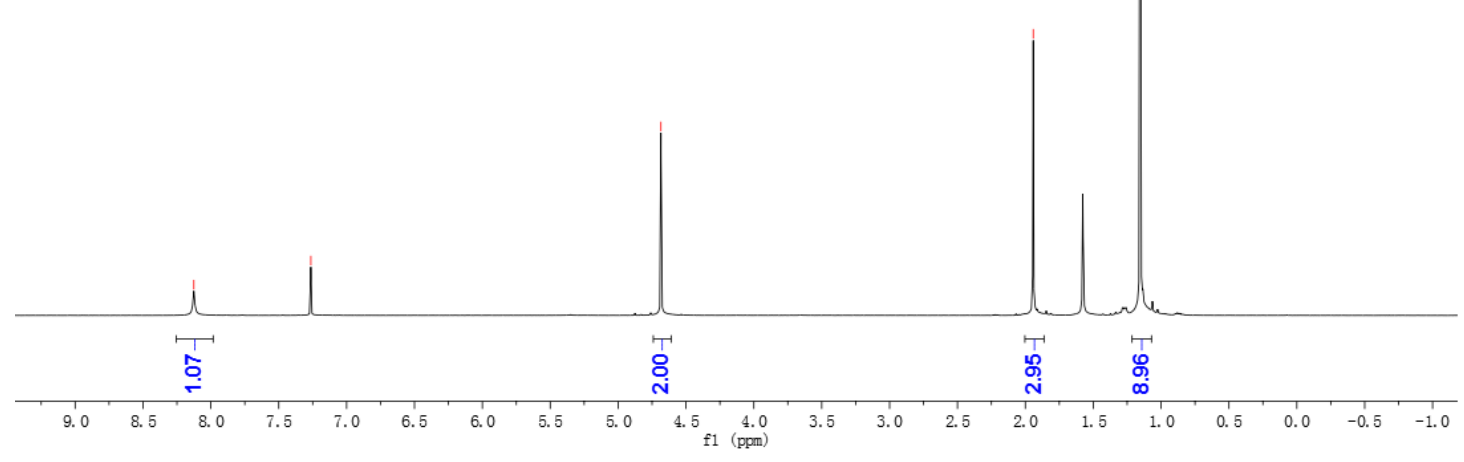

${ }^{13} \mathrm{C}$ NMR (126 MHz, $\mathrm{CDCl}_{3}$ ) spectra for 1a

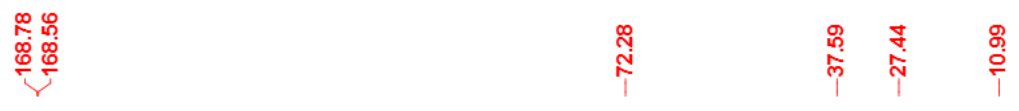<smiles>CC(=NOCC(=O)Nc1c(F)c(F)c(C(F)(F)F)c(F)c1F)C(C)(C)C</smiles>

$1 \mathbf{a}$

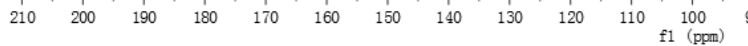


${ }^{1} \mathrm{H}$ NMR (500 MHz, $\left.\mathrm{CDCl}_{3}\right)$ spectra for $1 \mathrm{~b}$

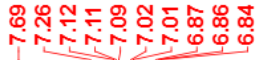<smiles>C/C=C\C=C/C(=O)Nc1c(F)c(F)c(F)c(C(F)(F)F)c1F</smiles><smiles>CN=C(C)C(C)(C)Nc1ccccc1</smiles>

1b
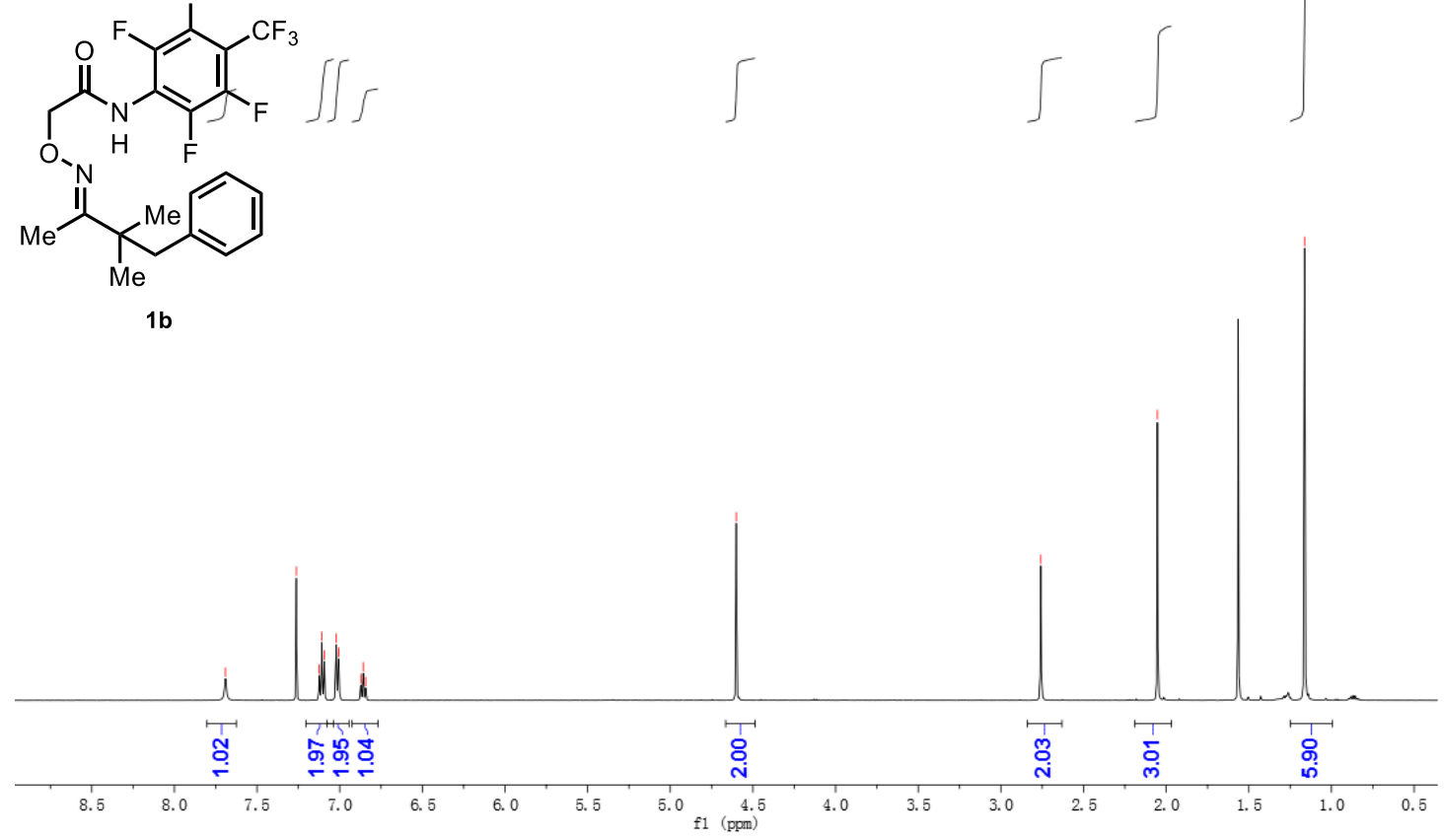

${ }^{13} \mathrm{C}$ NMR (126 MHz, $\mathrm{CDCl}_{3}$ ) spectra for $1 \mathrm{~b}$

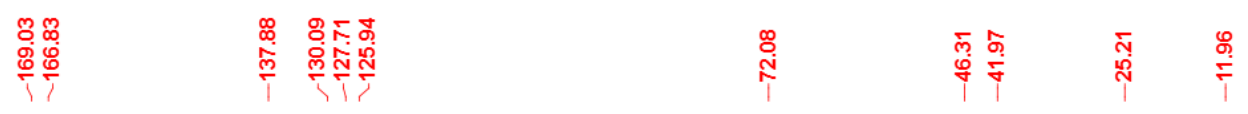<smiles>C/C(=N/OCC(=O)Nc1c(F)c(F)c(C(F)(F)F)c(F)c1F)C(C)(C)c1ccccc1</smiles>

$1 \mathrm{~b}$ ${ }_{\mathrm{f} 1}^{100}(\mathrm{ppm})$ 
${ }^{1} \mathrm{H}$ NMR (500 MHz, $\left.\mathrm{CDCl}_{3}\right)$ spectra for 1c

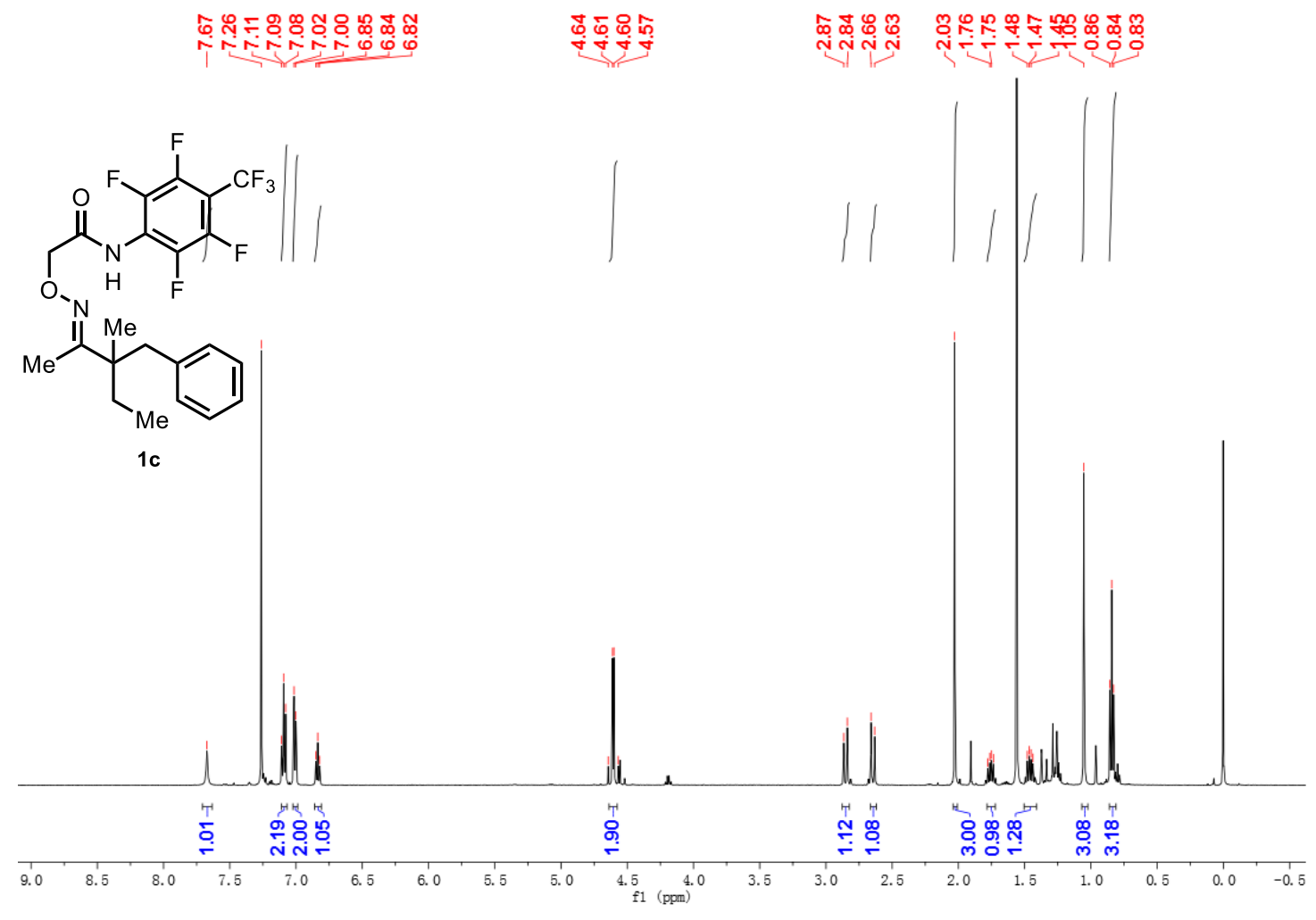

${ }^{13} \mathrm{C}$ NMR (126 $\left.\mathrm{MHz}, \mathrm{CDCl}_{3}\right)$ spectra for $1 \mathrm{c}$

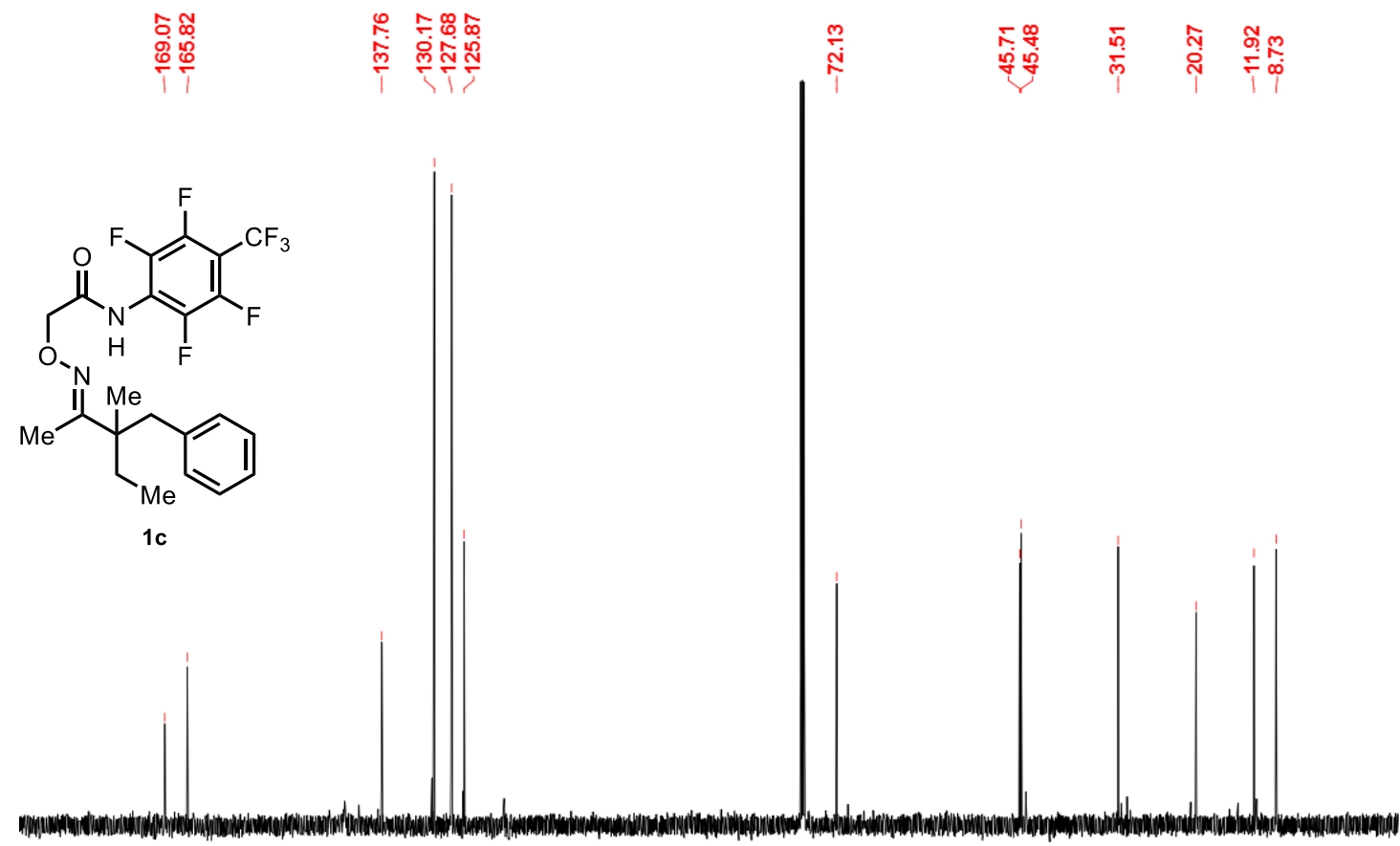

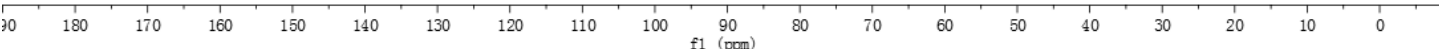


${ }^{1} \mathrm{H}$ NMR (500 MHz, $\left.\mathrm{CDCl}_{3}\right)$ spectra for $1 \mathrm{~d}$

冓<smiles>C#CC(F)(F)c1c(F)c(F)c(NC(=O)CON)c(F)c1F</smiles><smiles>C=CC</smiles><smiles>C=C(C)C(C)(C)CCc1ccccc1</smiles>

$1 d$

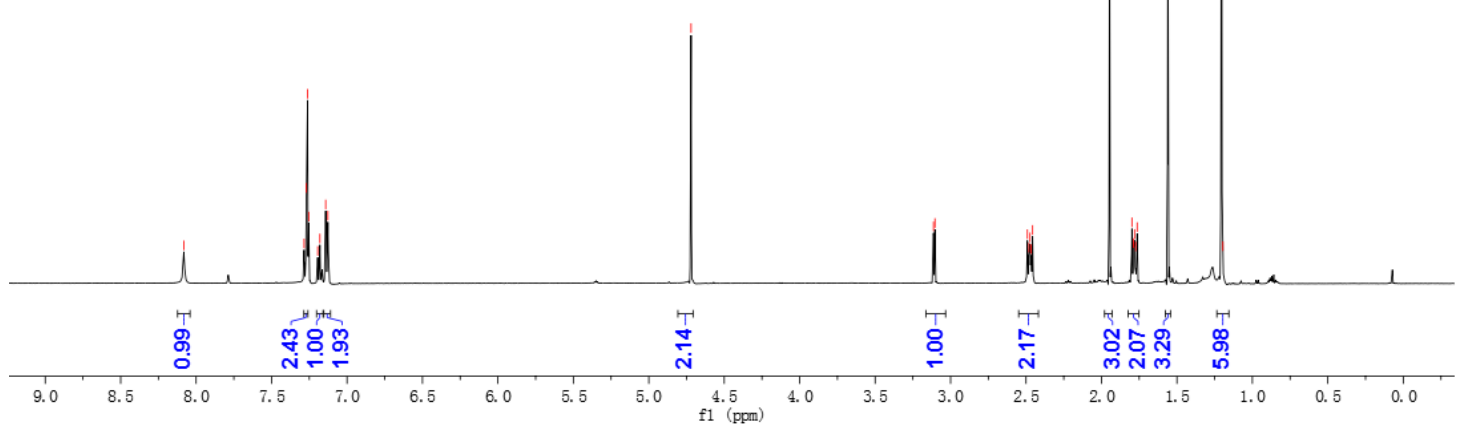

${ }^{13} \mathrm{C}$ NMR (126 MHz, $\left.\mathrm{CDCl}_{3}\right)$ spectra for $1 \mathrm{~d}$<smiles>C/C(=N/OCC(=O)Nc1c(F)c(F)c(C(F)(F)F)c(F)c1F)C(C)(C)CCc1ccccc1</smiles>

1d
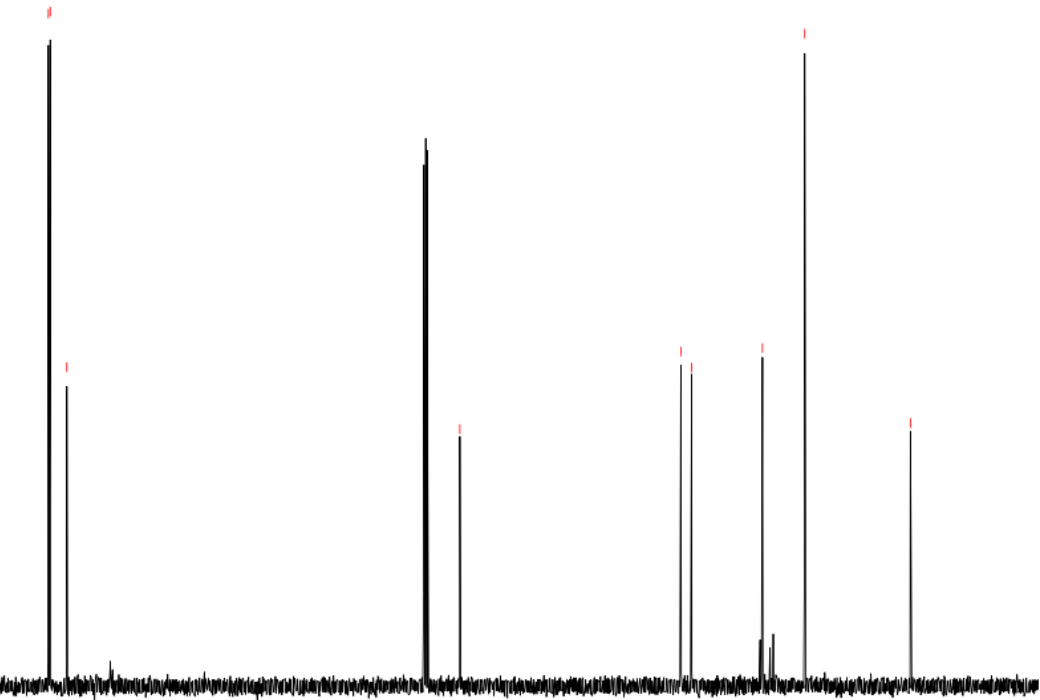

$30 \quad 180$

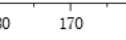

${ }_{150}^{1}$

$\begin{array}{lllll}130 & 120 & 110 & 100 & \substack{90 \\(\mathrm{ppm})}\end{array}$ 
${ }^{1} \mathrm{H}$ NMR (500 MHz, $\mathrm{CDCl}_{3}$ ) spectra for $1 \mathrm{e}$

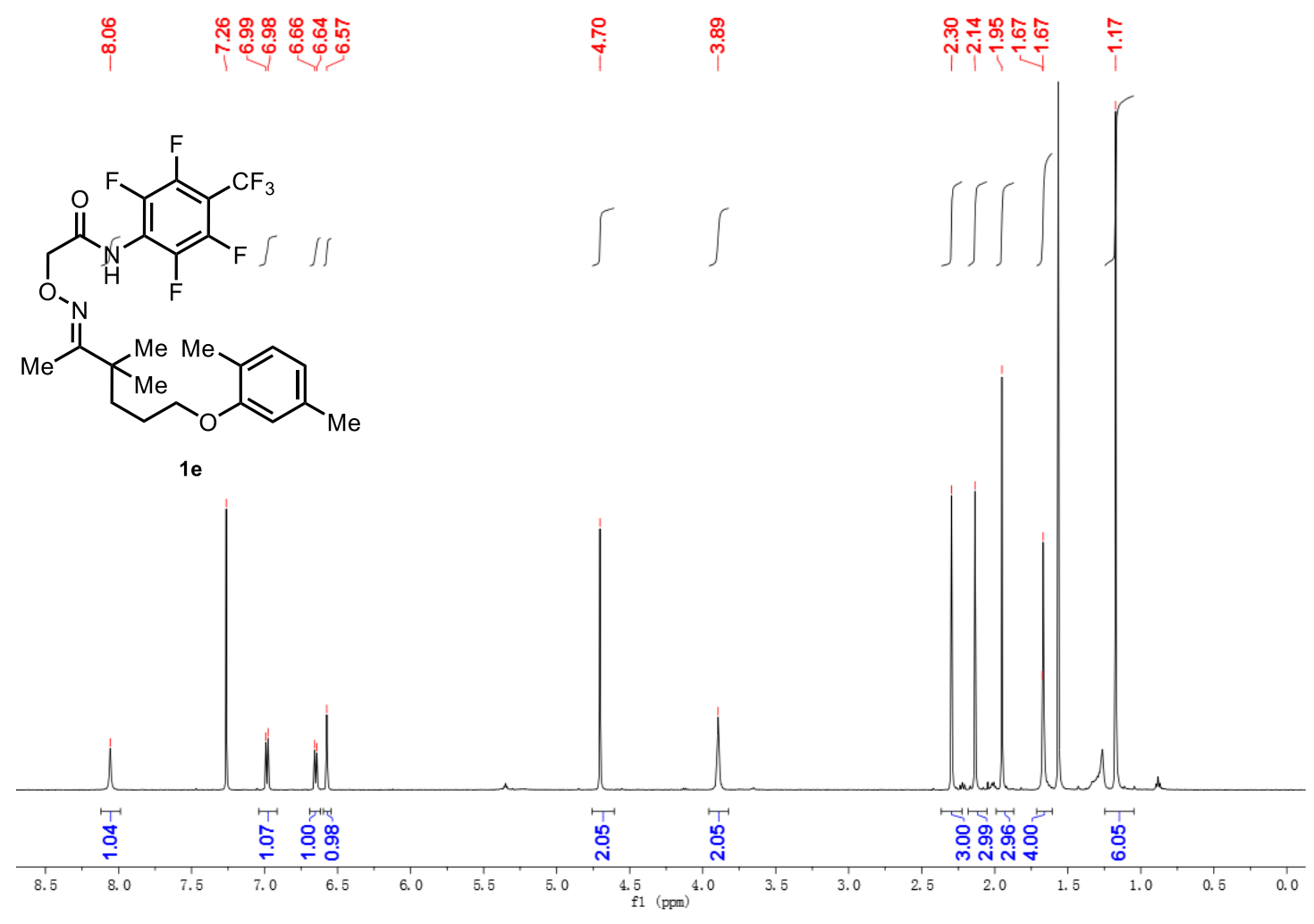

${ }^{13} \mathrm{C}$ NMR (126 MHz, $\mathrm{CDCl}_{3}$ ) spectra for $1 \mathrm{e}$

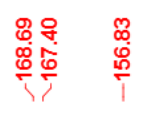

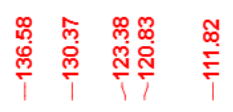
৫্ণ
용 照<smiles>NOCC(=O)Nc1c(F)c(F)c(C(F)(F)F)c(F)c1F</smiles><smiles>COc1ccc(C)cc1OCCCC(C)(C)C(C)=N</smiles>

1e
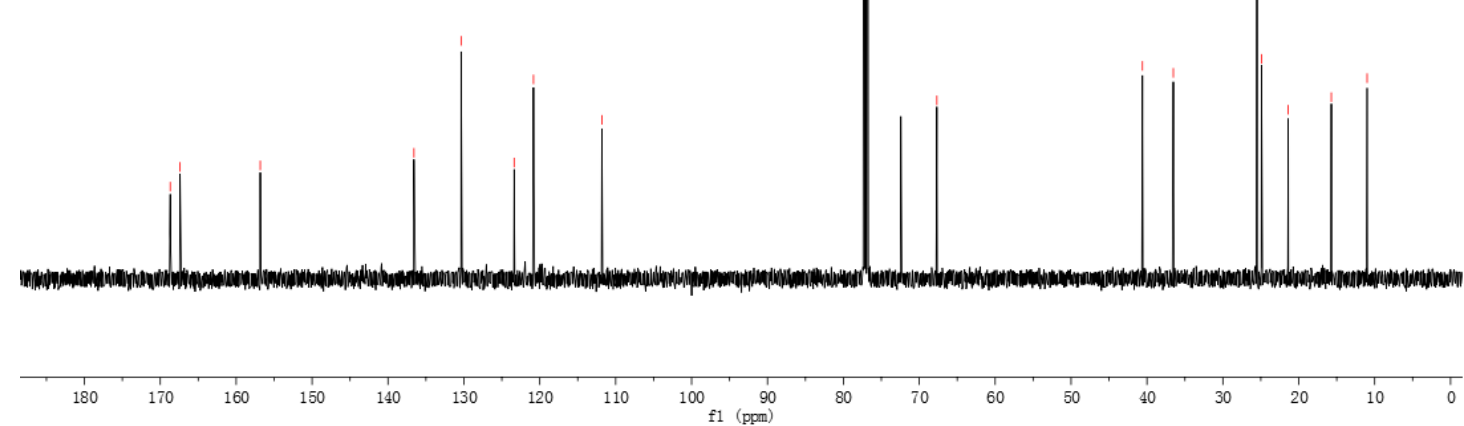
${ }^{1} \mathrm{H}$ NMR (500 MHz, $\left.\mathrm{CDCl}_{3}\right)$ spectra for $1 \mathrm{f}$

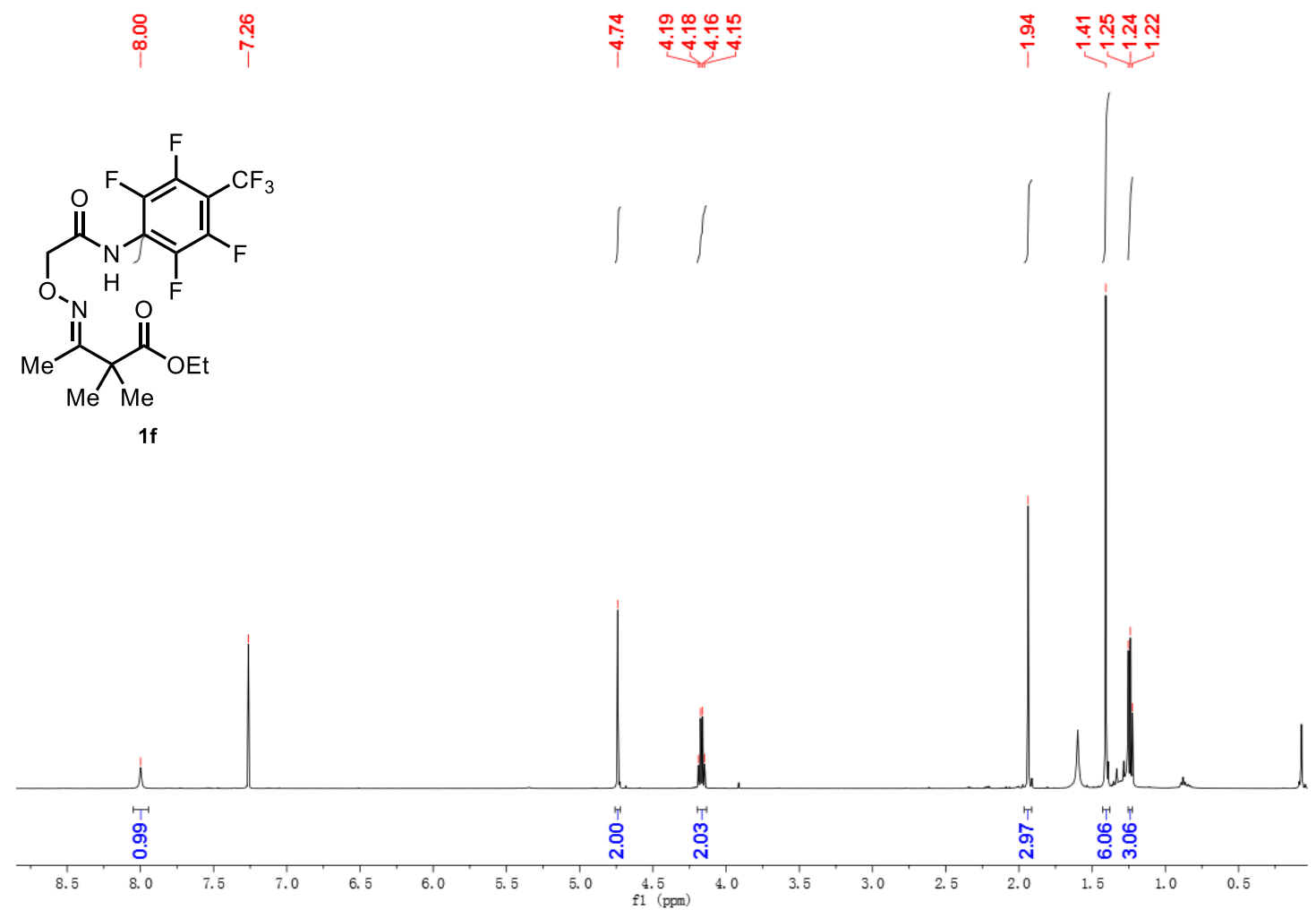

${ }^{13} \mathrm{C}$ NMR (126 MHz, $\mathrm{CDCl}_{3}$ ) spectra for $1 \mathrm{f}$

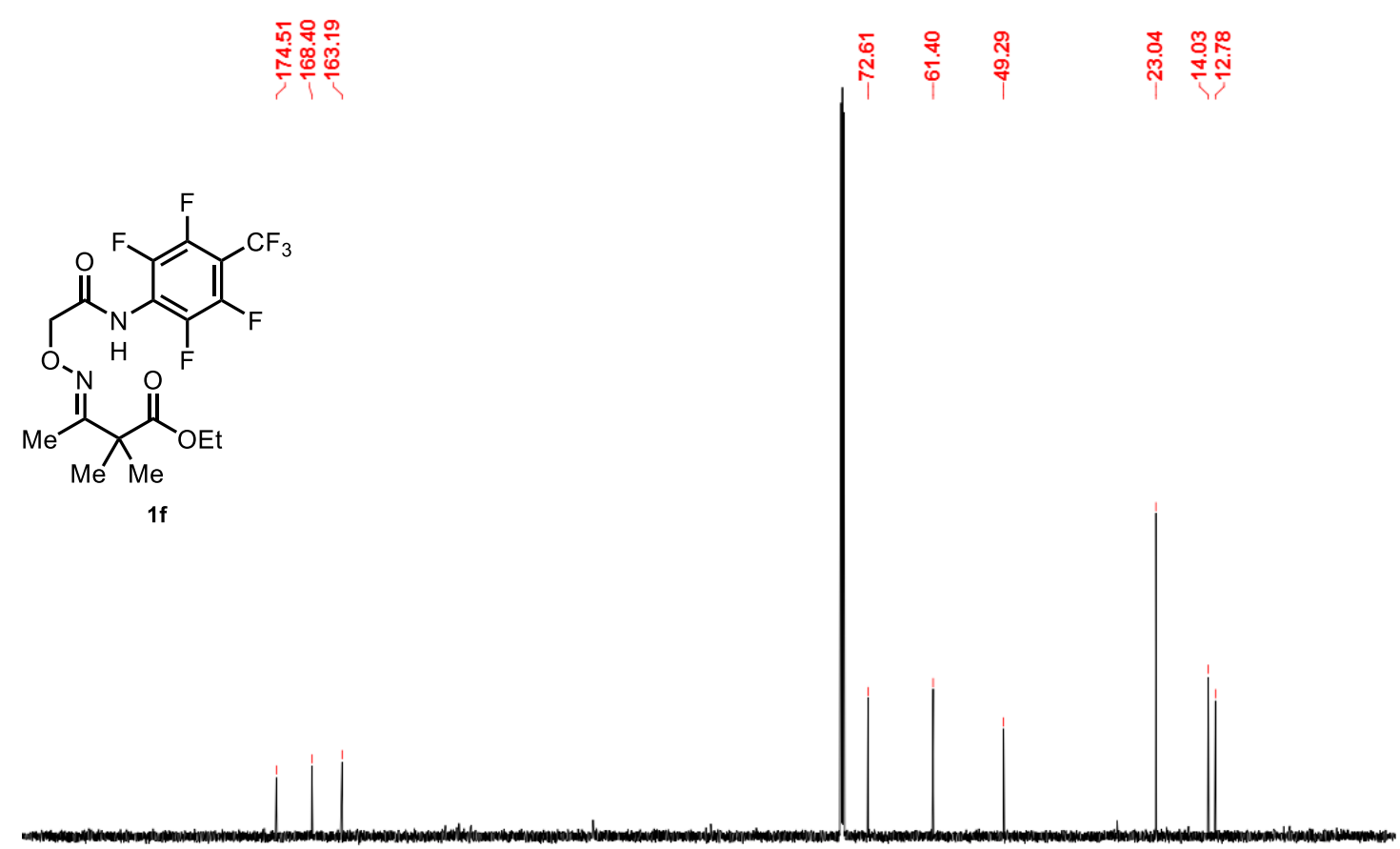

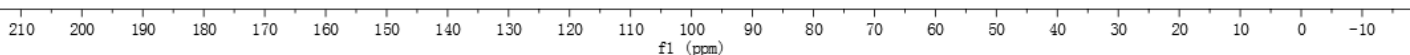


${ }^{1} \mathrm{H}$ NMR (500 MHz, $\mathrm{CDCl}_{3}$ ) spectra for $1 \mathrm{~g}$
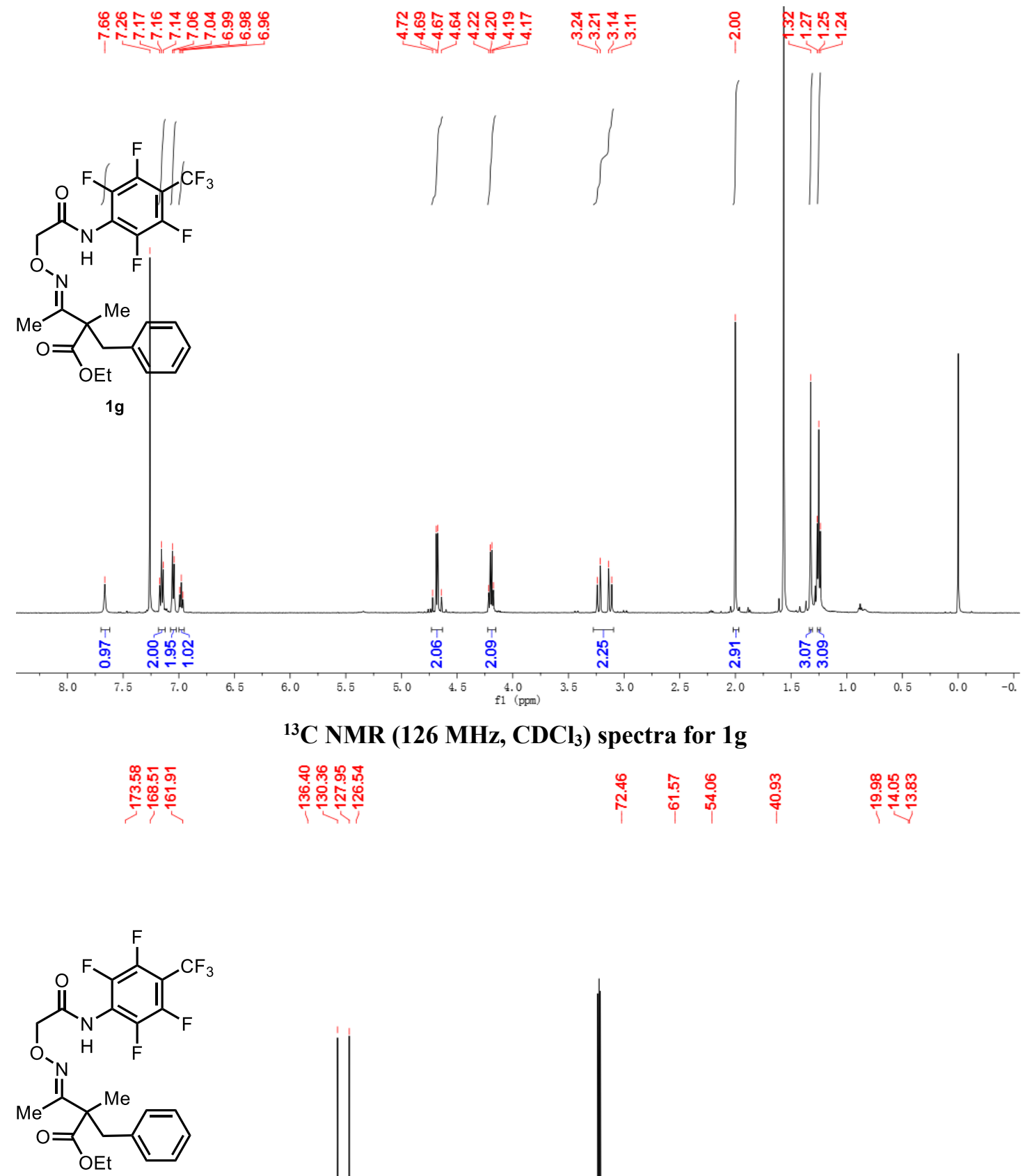

$1 \mathrm{~g}$

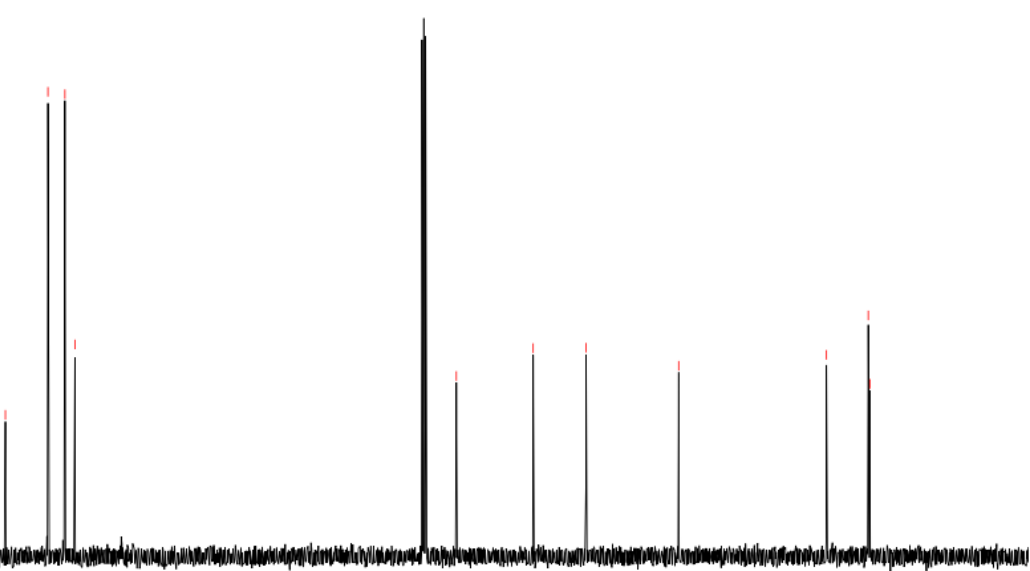

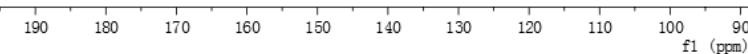


${ }^{1} \mathrm{H}$ NMR (500 MHz, CDCl $)$ spectra for $1 \mathrm{~h}$

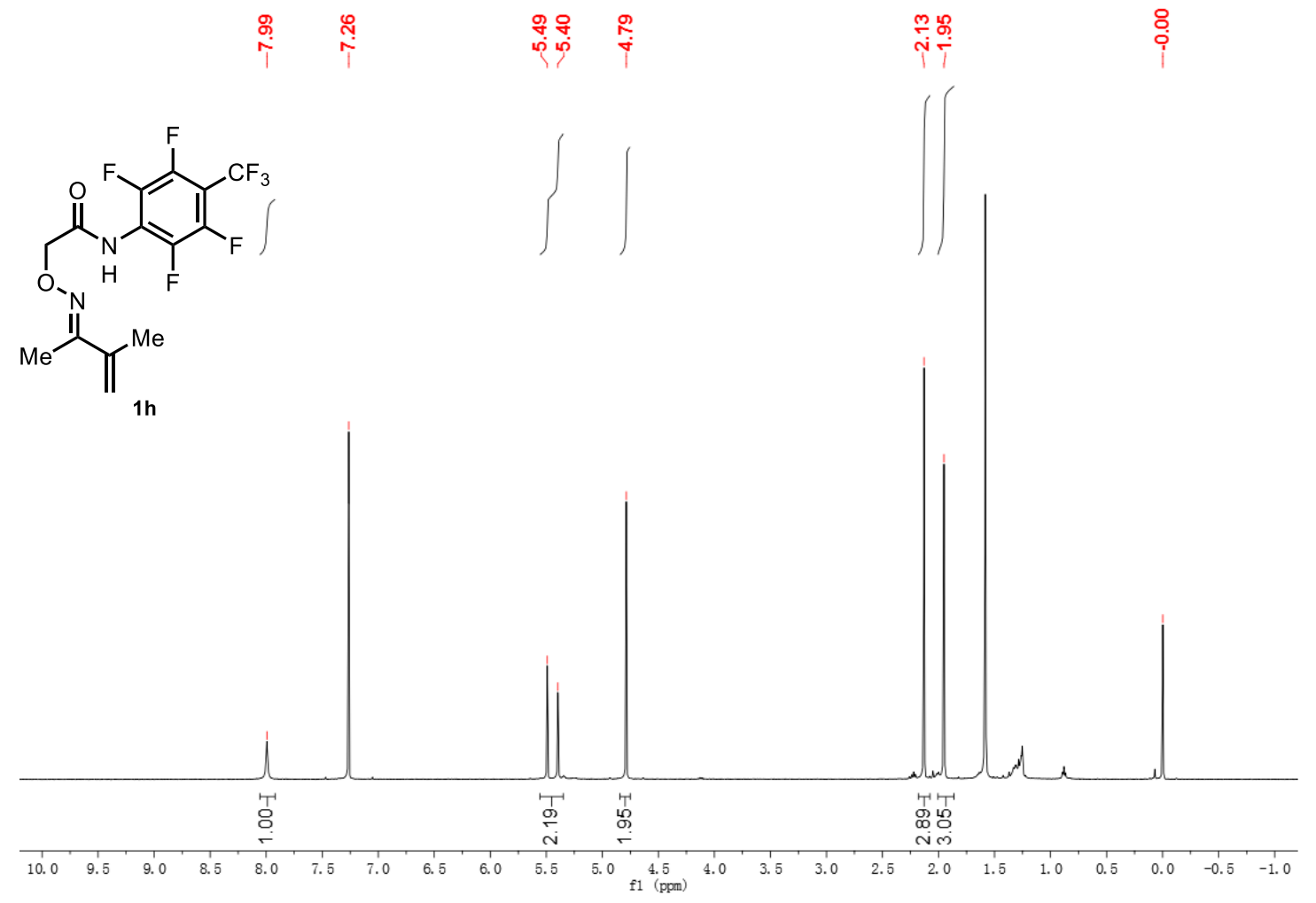

${ }^{13} \mathrm{C}$ NMR (126 MHz, $\left.\mathrm{CDCl}_{3}\right)$ spectra for $1 \mathrm{~h}$

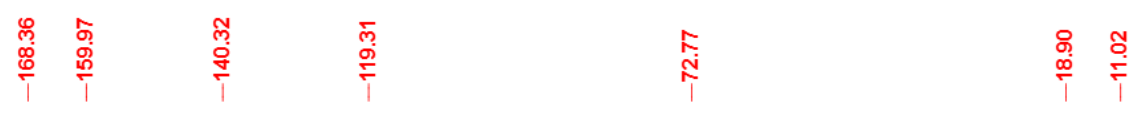

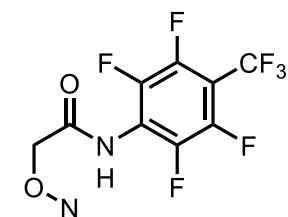

Me $\overbrace{}^{M e}$

$1 \mathrm{~h}$

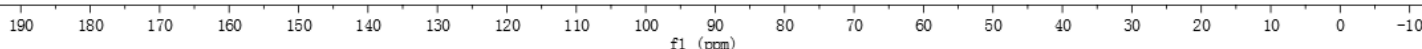


${ }^{1} \mathrm{H}$ NMR (500 MHz, $\mathrm{CDCl}_{3}$ ) spectra for $1 \mathrm{i}$
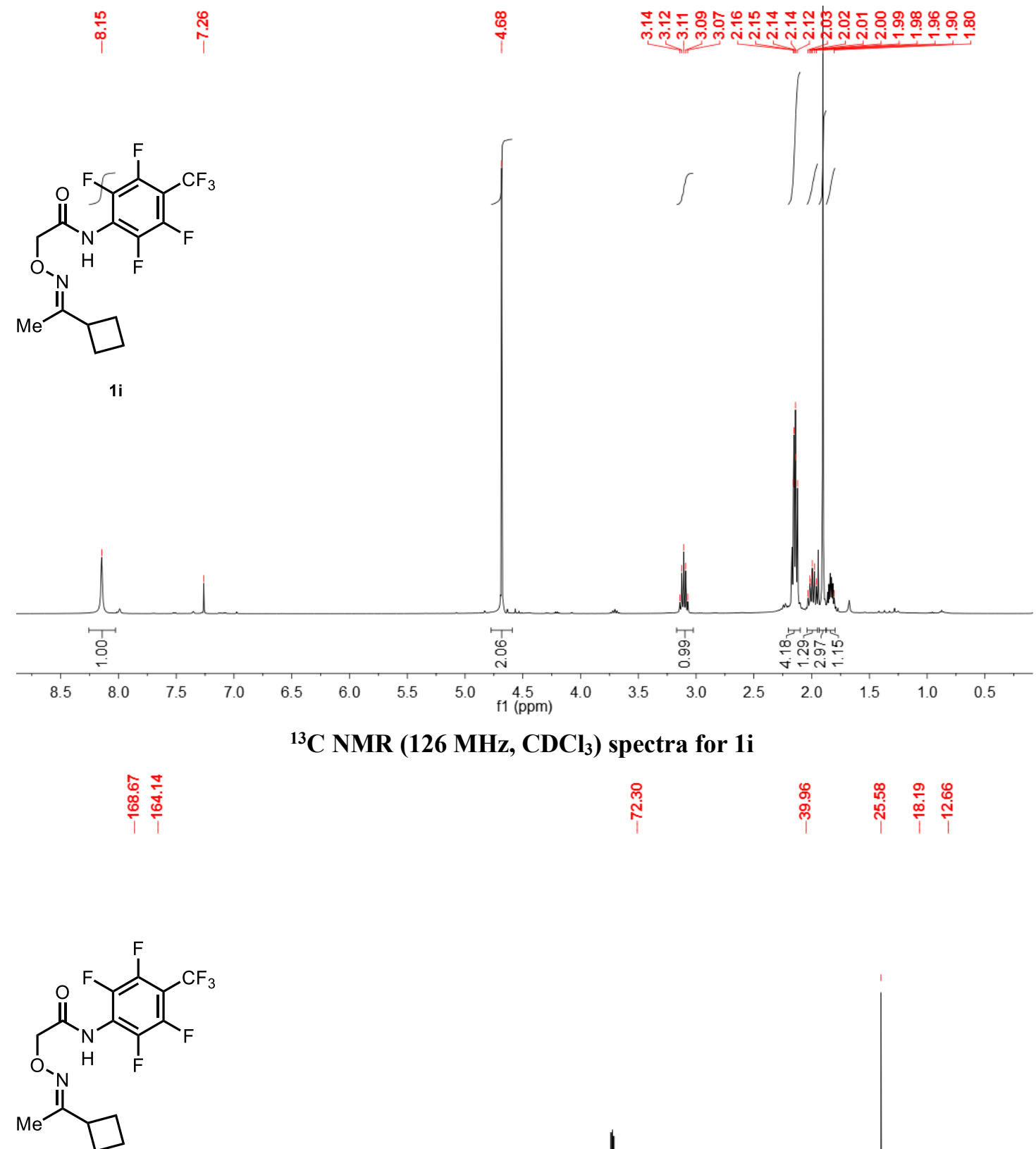

$1 \mathrm{i}$

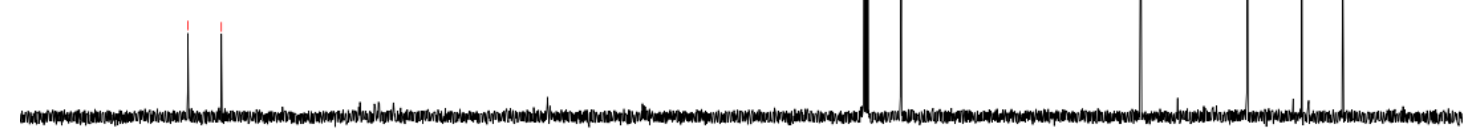

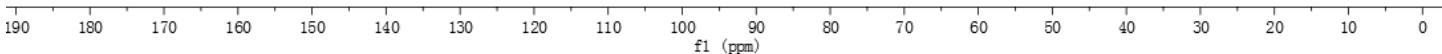




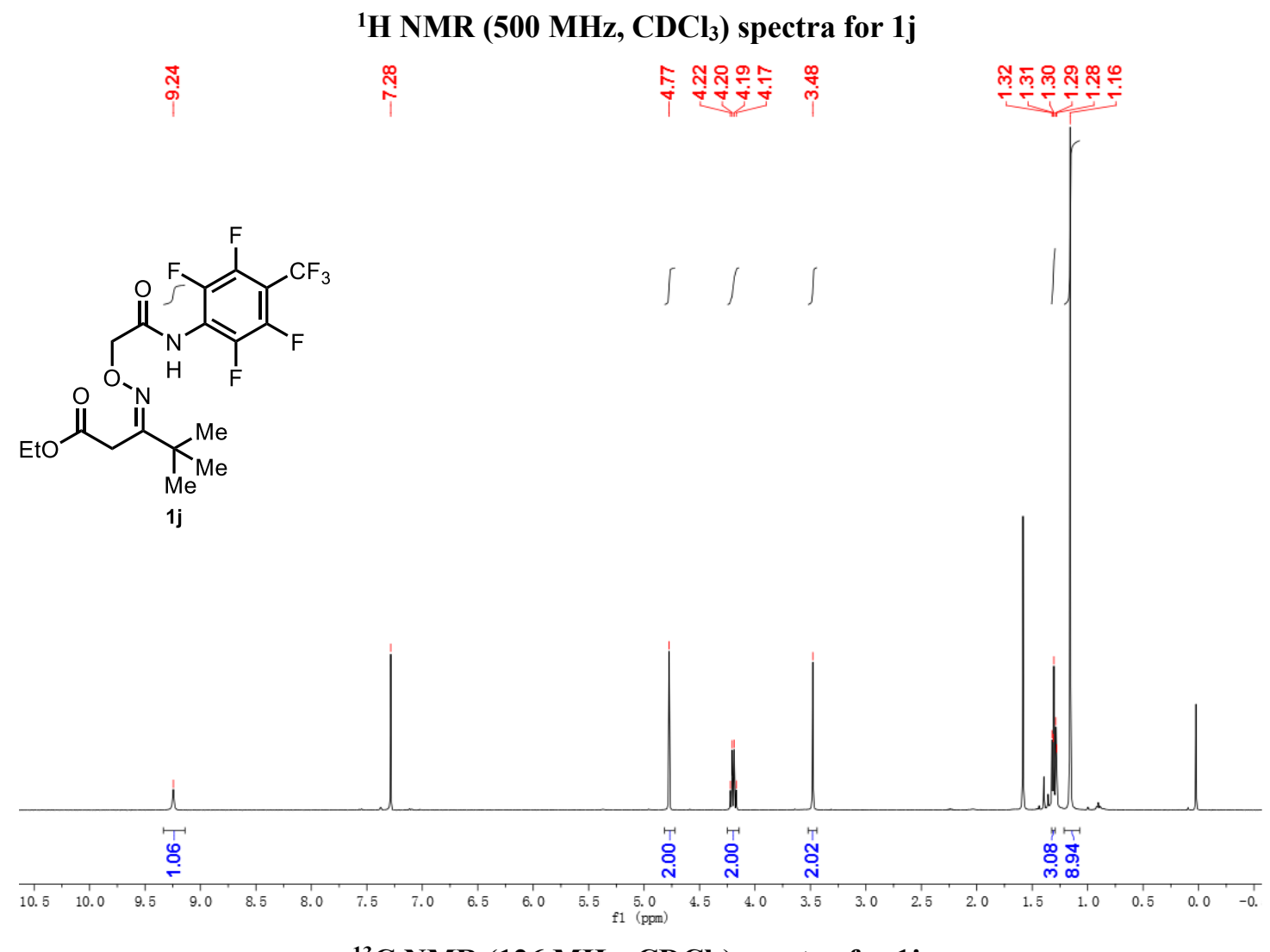

${ }^{13} \mathrm{C}$ NMR (126 MHz, $\left.\mathrm{CDCl}_{3}\right)$ spectra for $1 \mathrm{j}$

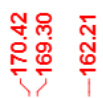

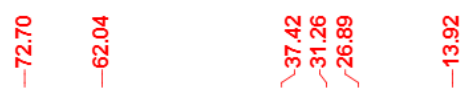<smiles>CCOC(=O)C/C(=N\OCC(=O)Nc1c(F)c(F)c(C(F)(F)F)c(F)c1F)C(C)(C)C</smiles>

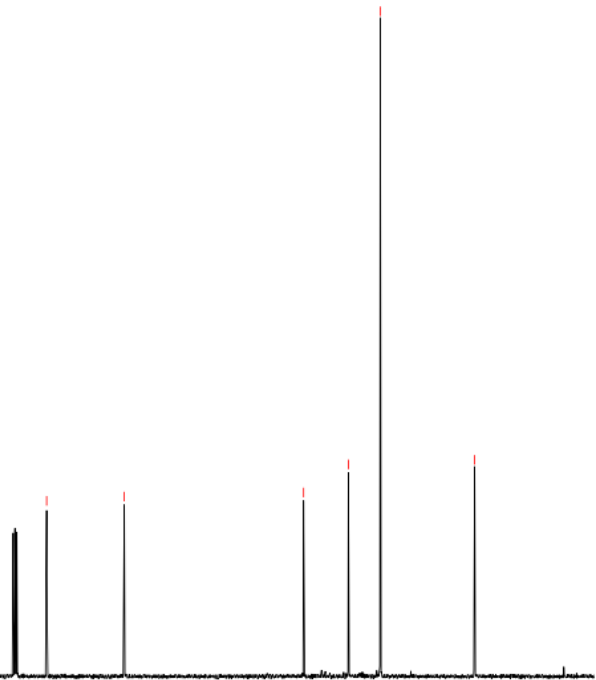

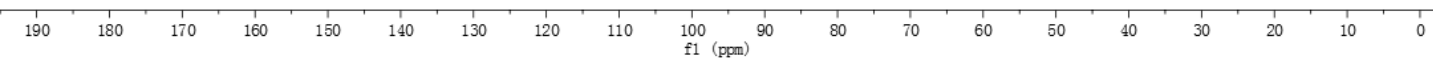


${ }^{1} \mathrm{H}$ NMR (500 MHz, $\left.\mathrm{CDCl}_{3}\right)$ spectra for $1 \mathrm{k}$

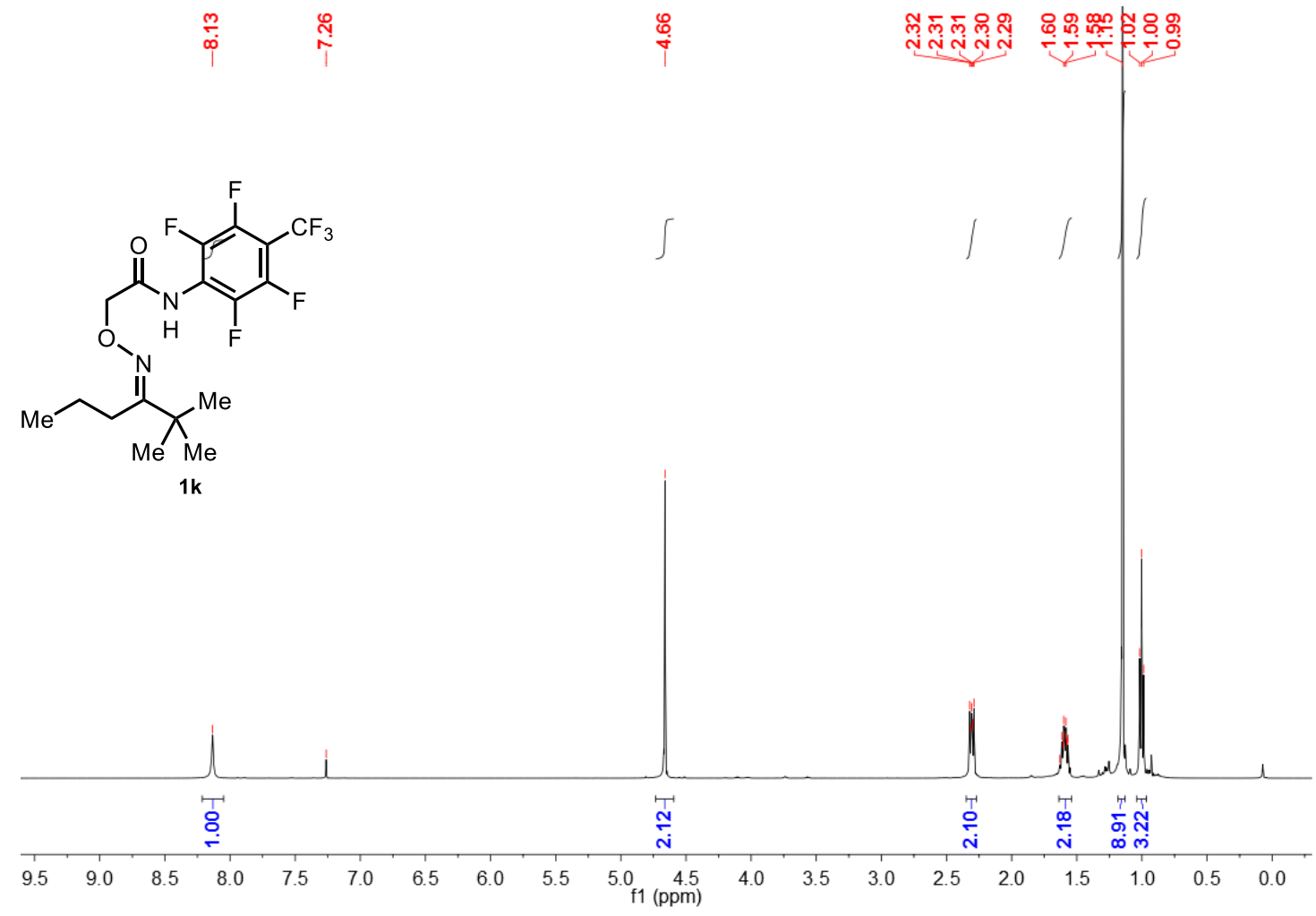

${ }^{13} \mathrm{C}$ NMR (126 MHz, $\mathrm{CDCl}_{3}$ ) spectra for $1 \mathrm{k}$
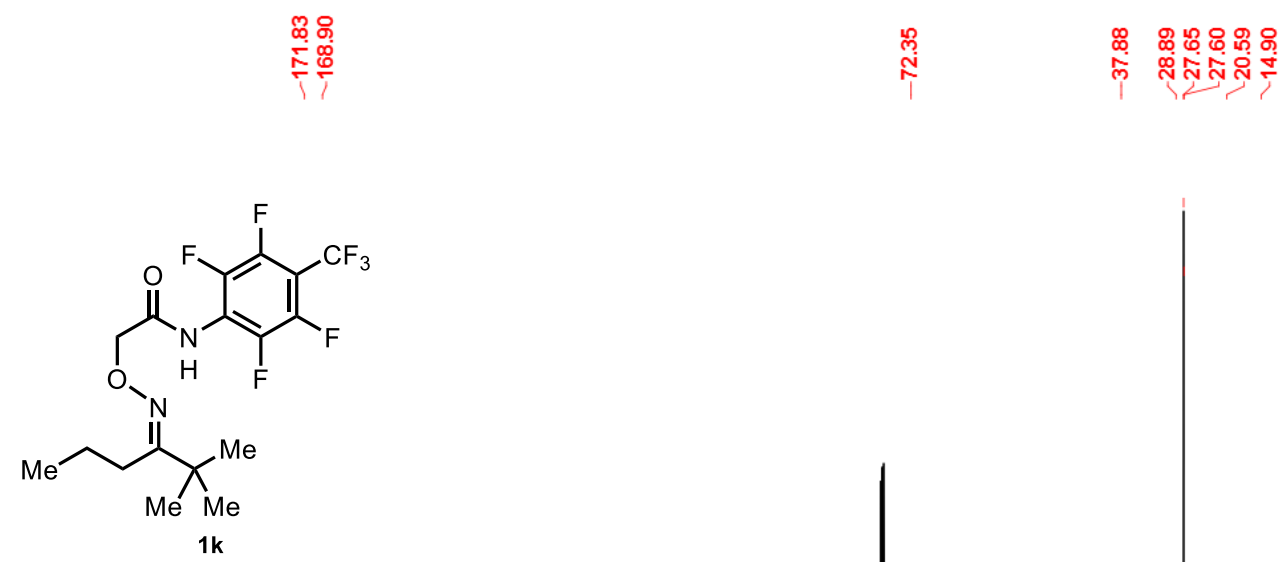

$\begin{array}{lllllllllllllllllllllllll}210 & 200 & 190 & 180 & 170 & 160 & 150 & 140 & 130 & 120 & 110 & 100 & 90 & 80 & 70 & 60 & 50 & 40 & 30 & 20 & 10 & 0 & -10\end{array}$ 
${ }^{1} \mathrm{H}$ NMR (500 MHz, $\mathrm{CDCl}_{3}$ ) spectra for 11

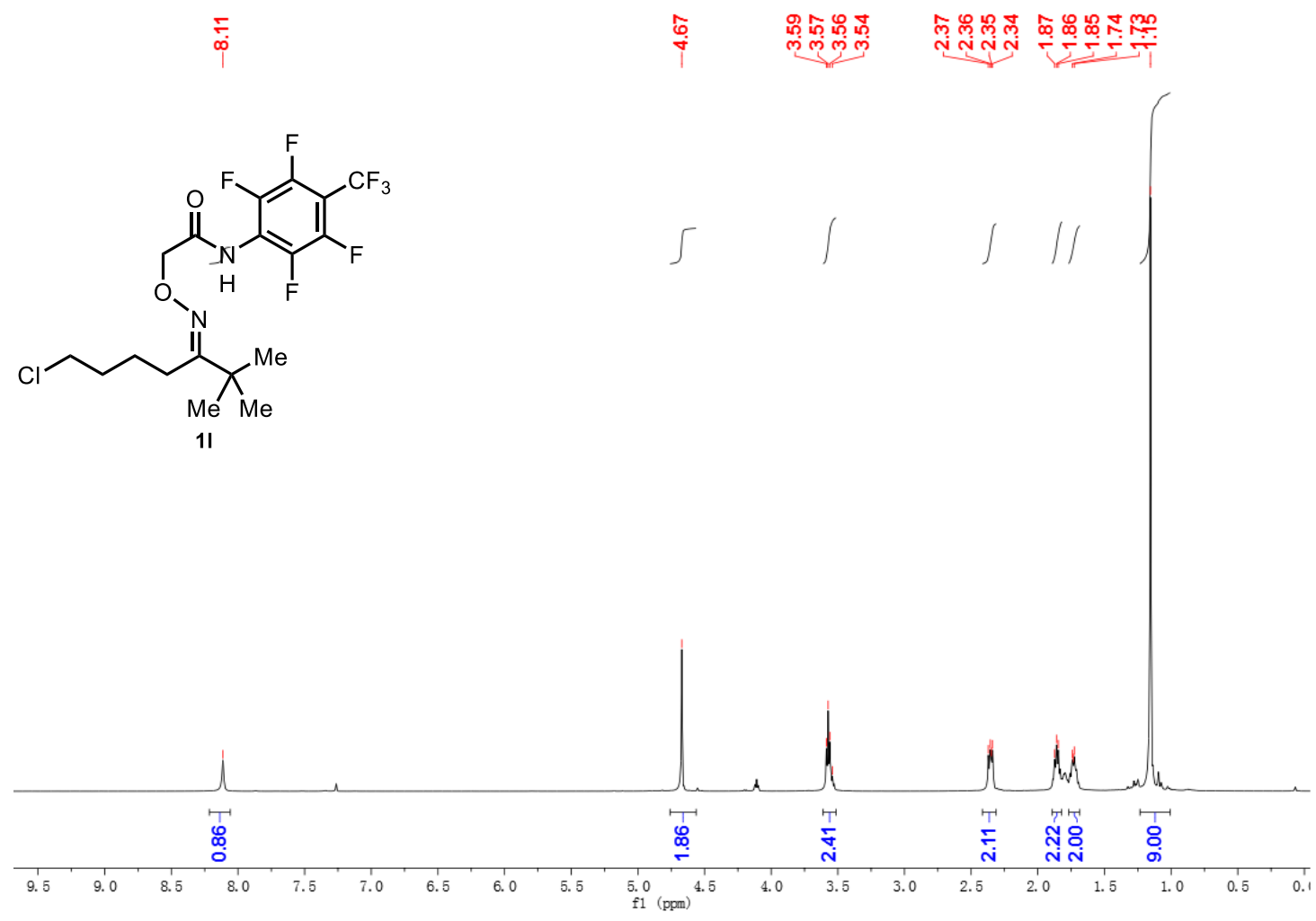

${ }^{13} \mathrm{C}$ NMR (126 MHz, $\mathrm{CDCl}_{3}$ ) spectra for 11

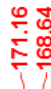

$\stackrel{\substack{m \\ i}}{i}$

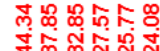

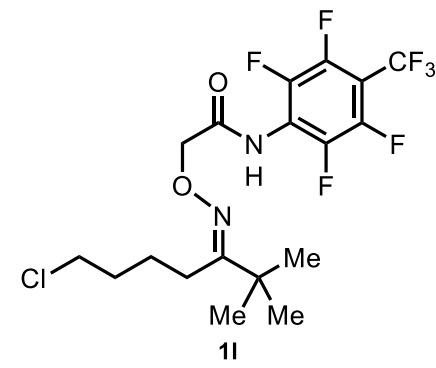

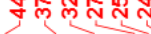

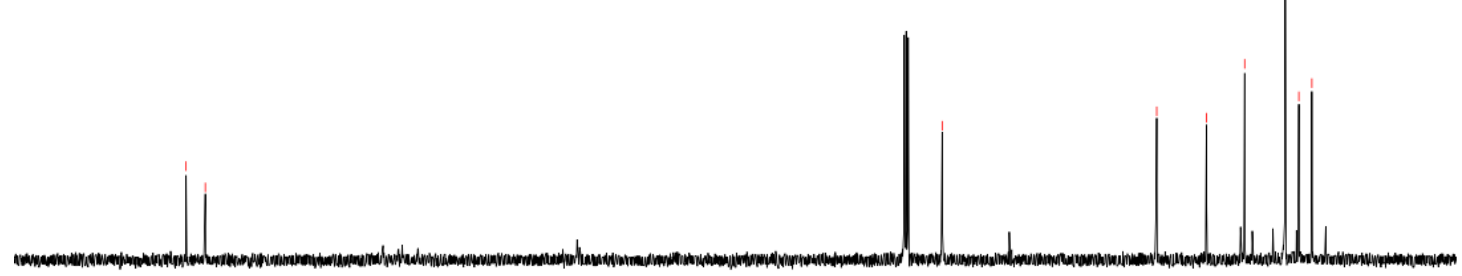

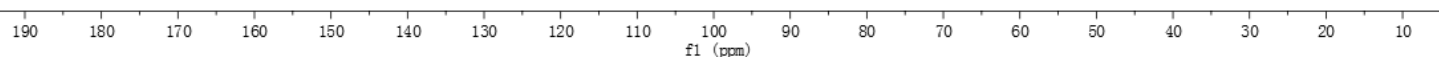


${ }^{1} \mathrm{H}$ NMR (500 MHz, $\mathrm{CDCl}_{3}$ ) spectra for $1 \mathrm{~m}$

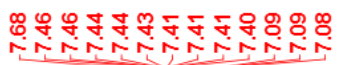

$\stackrel{\leftrightarrow}{\leftrightarrow}$<smiles>CC(C)(C)/C(=N\OCC(=O)Nc1c(F)c(F)c(C(F)(F)F)c(F)c1F)c1ccccc1</smiles>

$1 \mathrm{~m}$

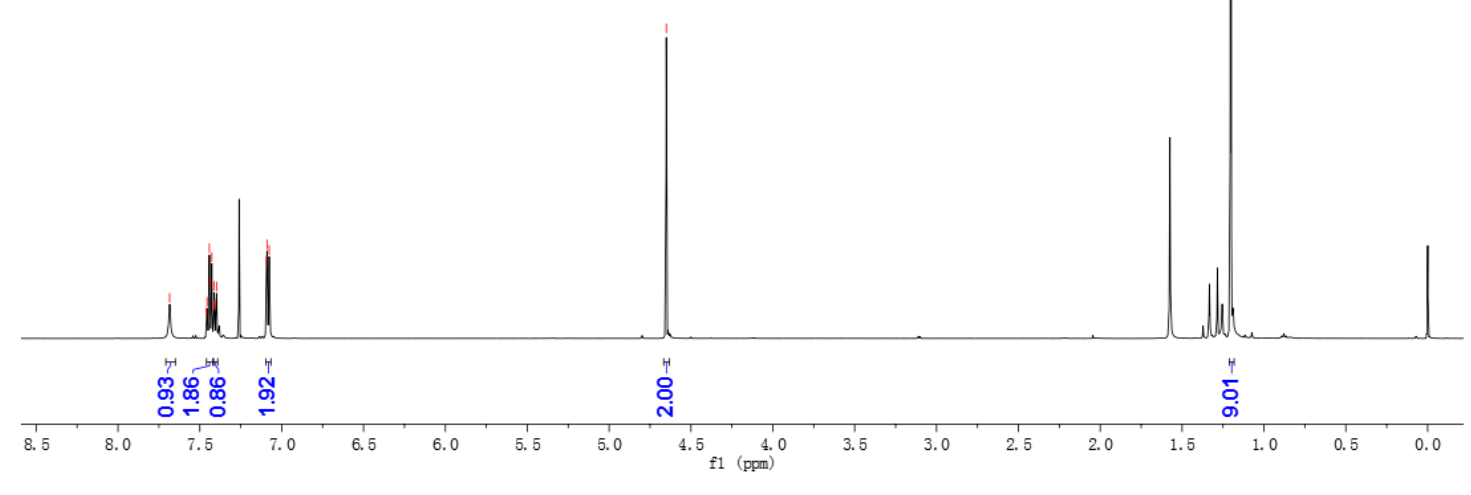

${ }^{13} \mathrm{C}$ NMR (126 MHz, $\mathrm{CDCl}_{3}$ ) spectra for $1 \mathrm{~m}$

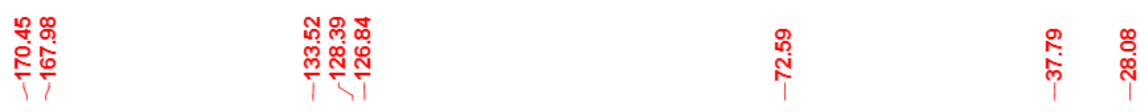<smiles>CC(C)(C)/C(=C\OCC(=O)Nc1c(F)c(F)c(C(F)(F)F)c(F)c1F)c1ccccc1</smiles>

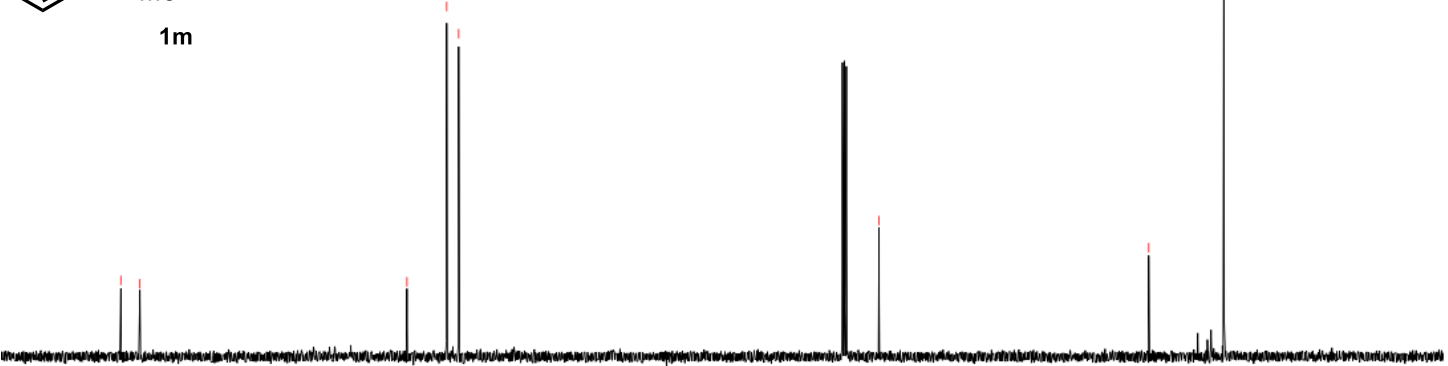

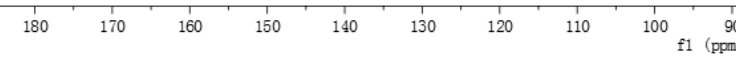


${ }^{1} \mathrm{H}$ NMR (500 MHz, $\mathrm{CDCl}_{3}$ ) spectra for $1 \mathrm{n}$

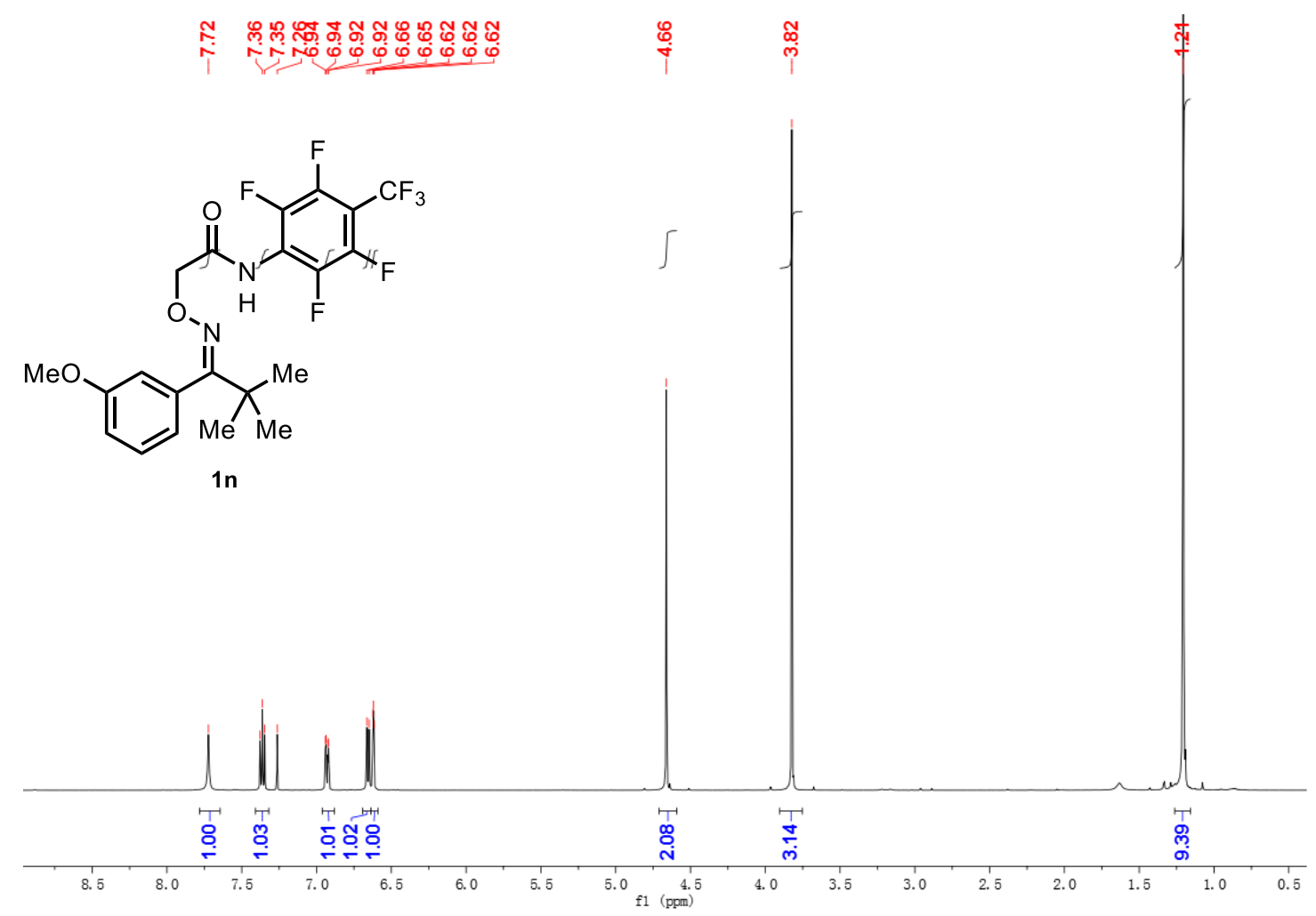

${ }^{13} \mathrm{C}$ NMR (126 MHz, $\left.\mathrm{CDCl}_{3}\right)$ spectra for $1 \mathrm{n}$

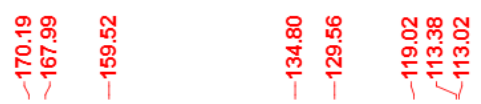<smiles>NOCC(=O)Nc1c(F)c(F)c(C(F)(F)F)c(F)c1F</smiles><smiles>COc1cccc(C(=N)C(C)(C)C)c1</smiles>

1n

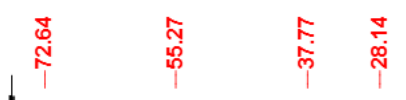
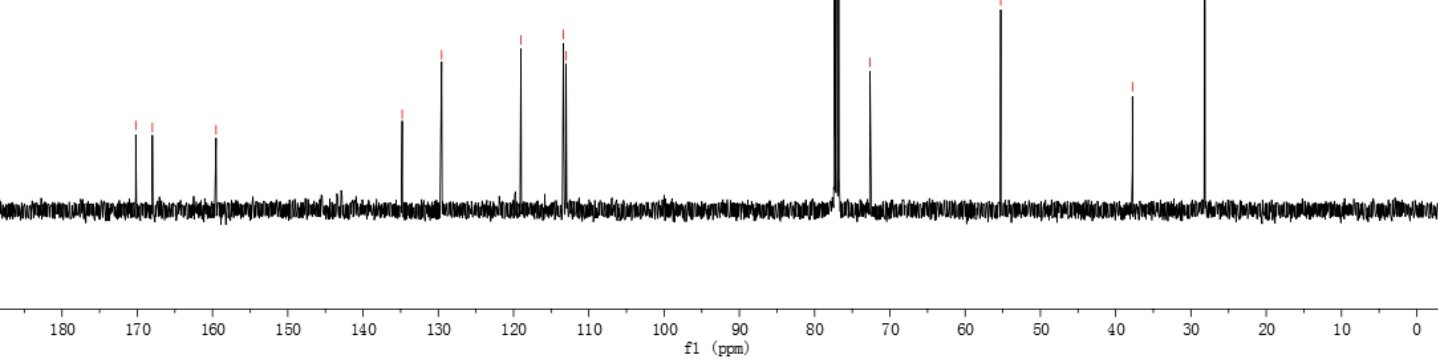
${ }^{1} \mathrm{H}$ NMR (500 MHz, $\left.\mathrm{CDCl}_{3}\right)$ spectra for 10

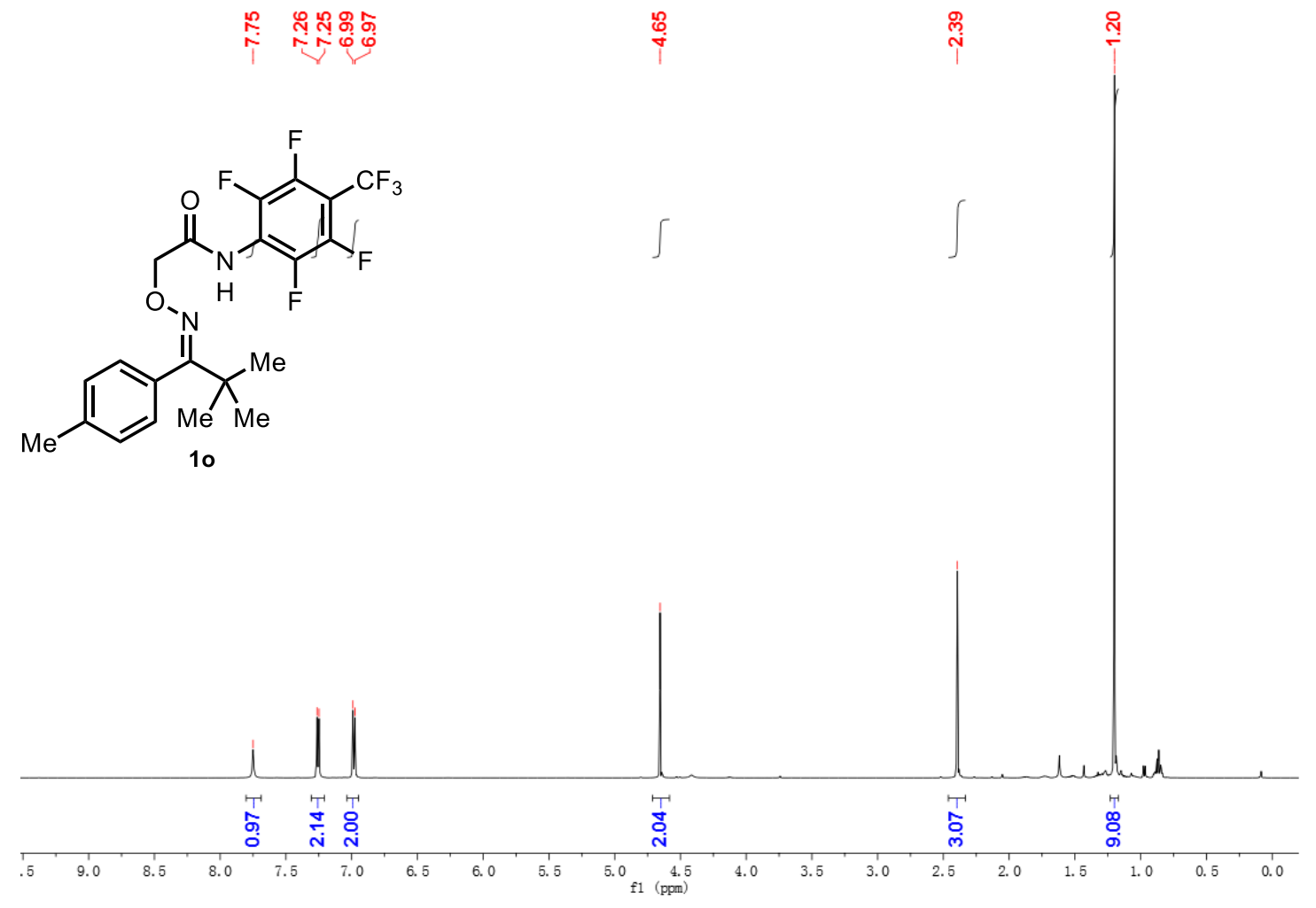

${ }^{13} \mathrm{C}$ NMR (126 MHz, $\left.\mathrm{CDCl}_{3}\right)$ spectra for 10

\begin{tabular}{|c|c|c|c|}
\hline 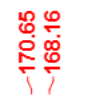 & 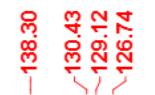 & $\stackrel{\text { Sి }}{\text { N }}$ & 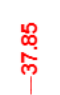 \\
\hline
\end{tabular}
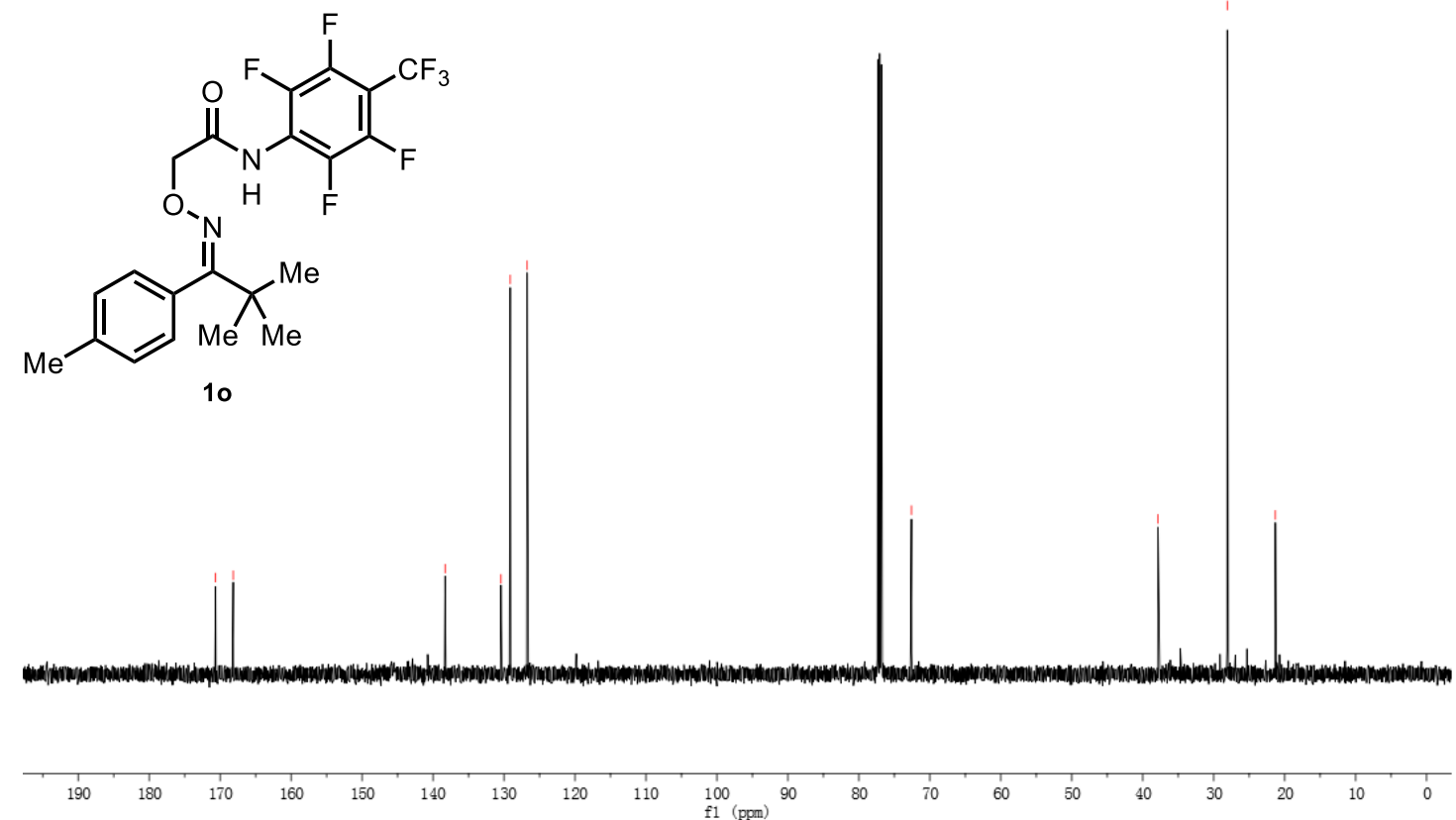
${ }^{1} \mathrm{H}$ NMR (500 MHz, $\left.\mathrm{CDCl}_{3}\right)$ spectra for $1 \mathrm{p}$

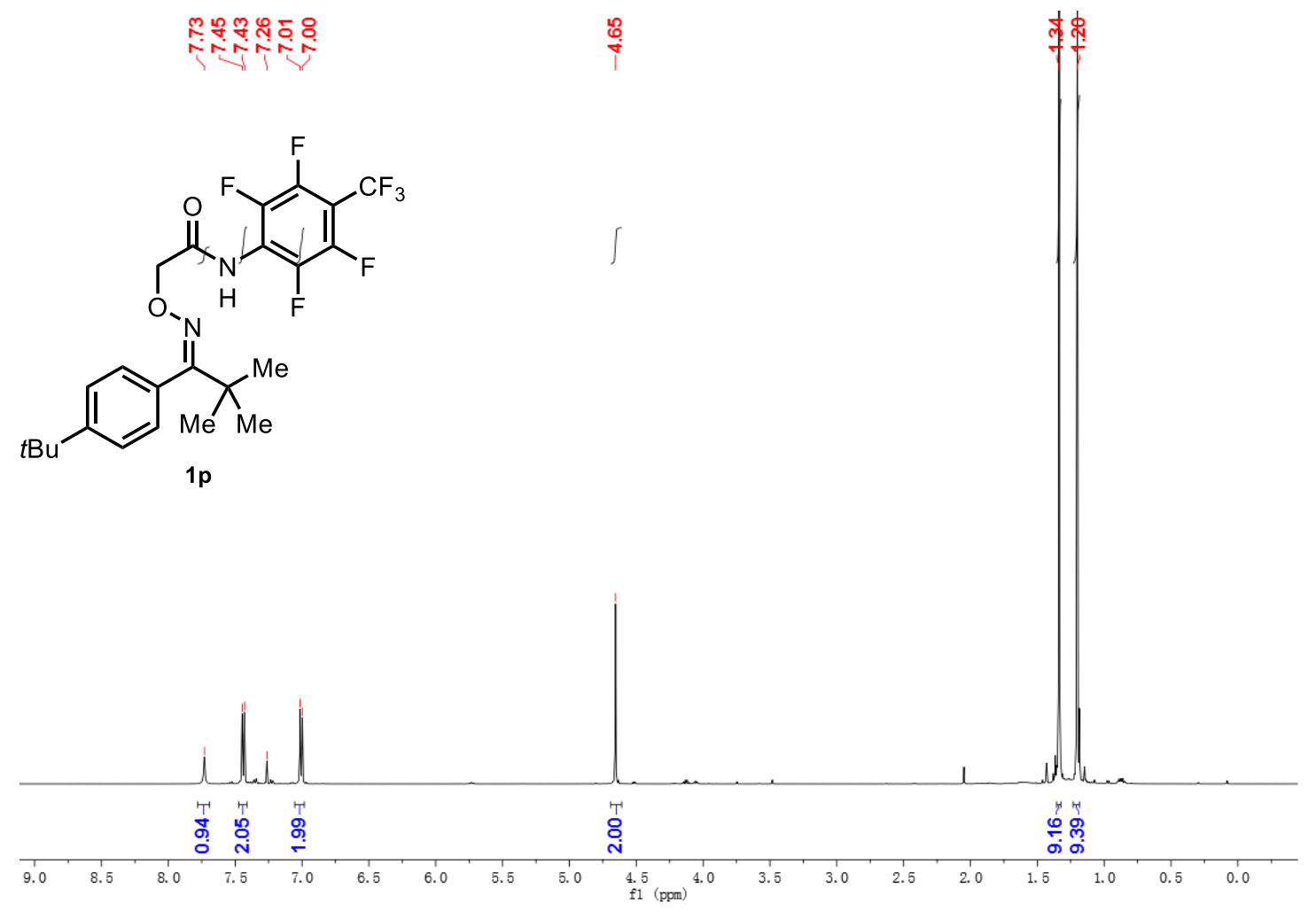

${ }^{13} \mathrm{C}$ NMR (126 MHz, $\left.\mathrm{CDCl}_{3}\right)$ spectra for $1 \mathrm{p}$

80
0

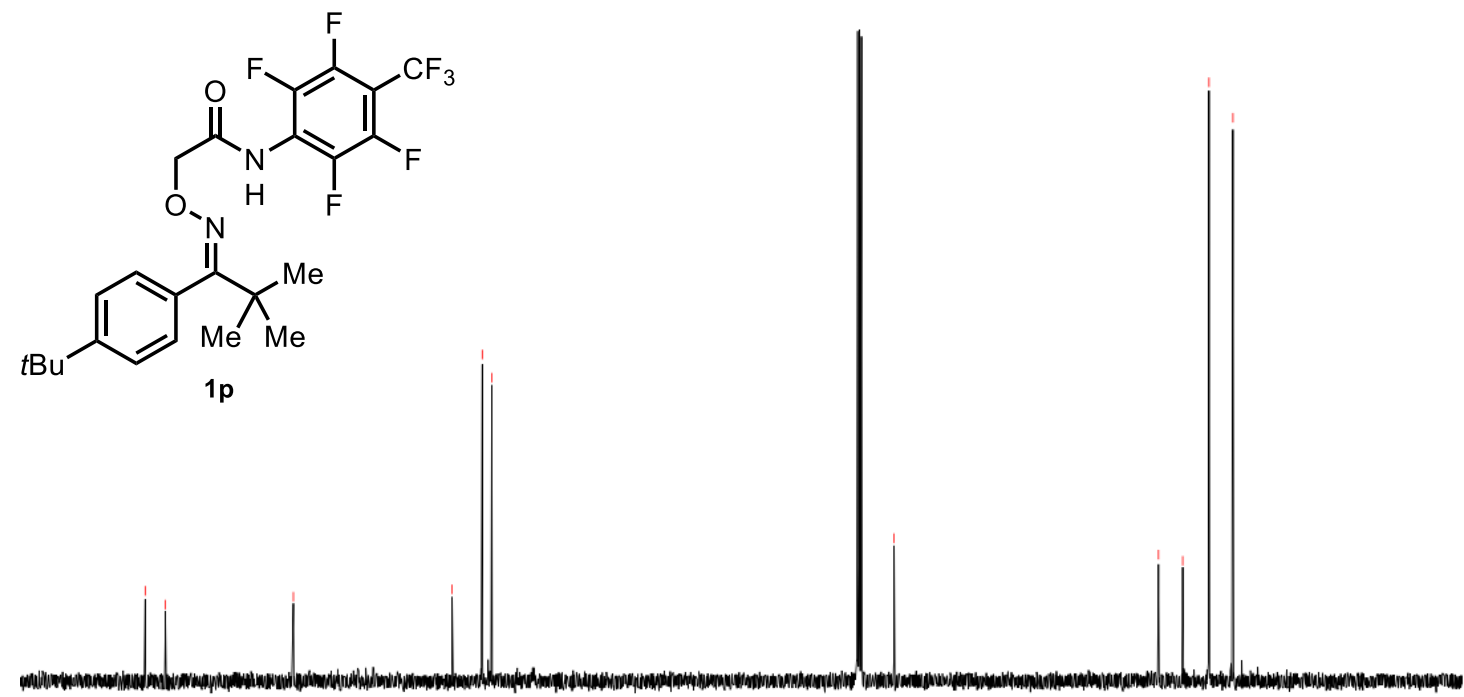

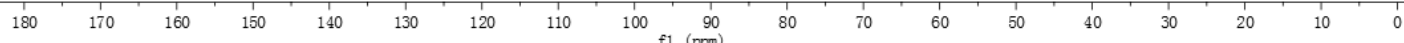


${ }^{1} \mathrm{H}$ NMR (500 MHz, $\left.\mathrm{CDCl}_{3}\right)$ spectra for $1 \mathrm{q}$

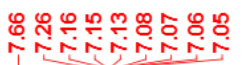
芦<smiles>O=C(CONc1c(F)c(F)c(C(F)(F)F)c(F)c1F)NCO</smiles><smiles>CN=C(NC)C(C)(C)C</smiles>

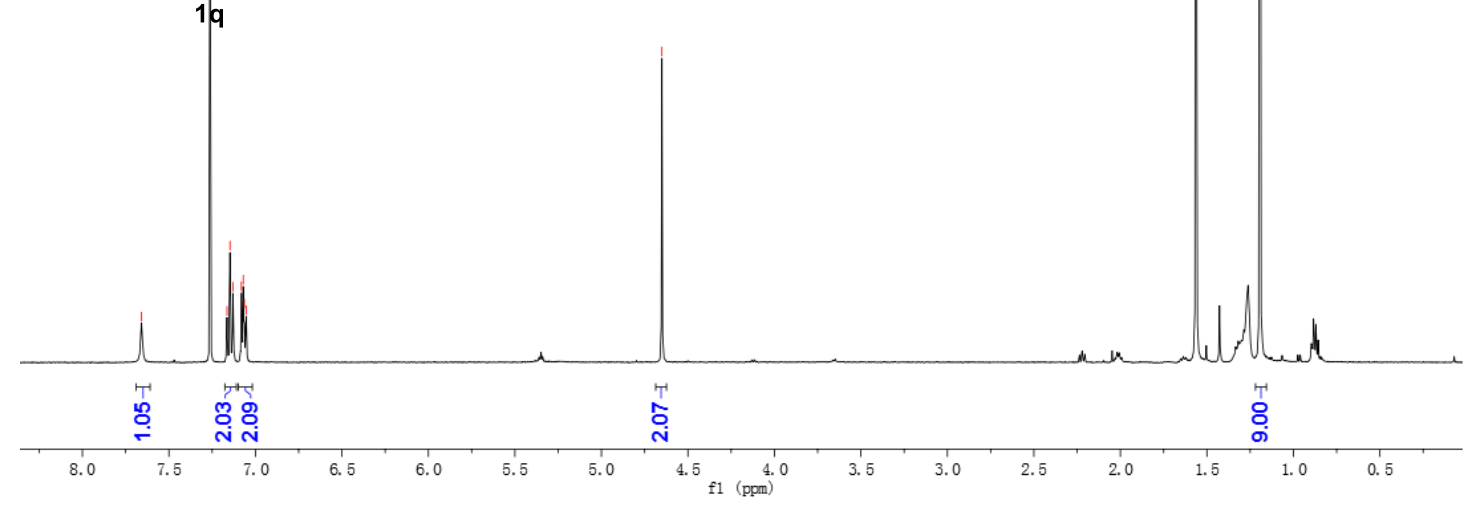

${ }^{13} \mathrm{C}$ NMR (126 MHz, $\left.\mathrm{CDCl}_{3}\right)$ spectra for $1 \mathrm{q}$

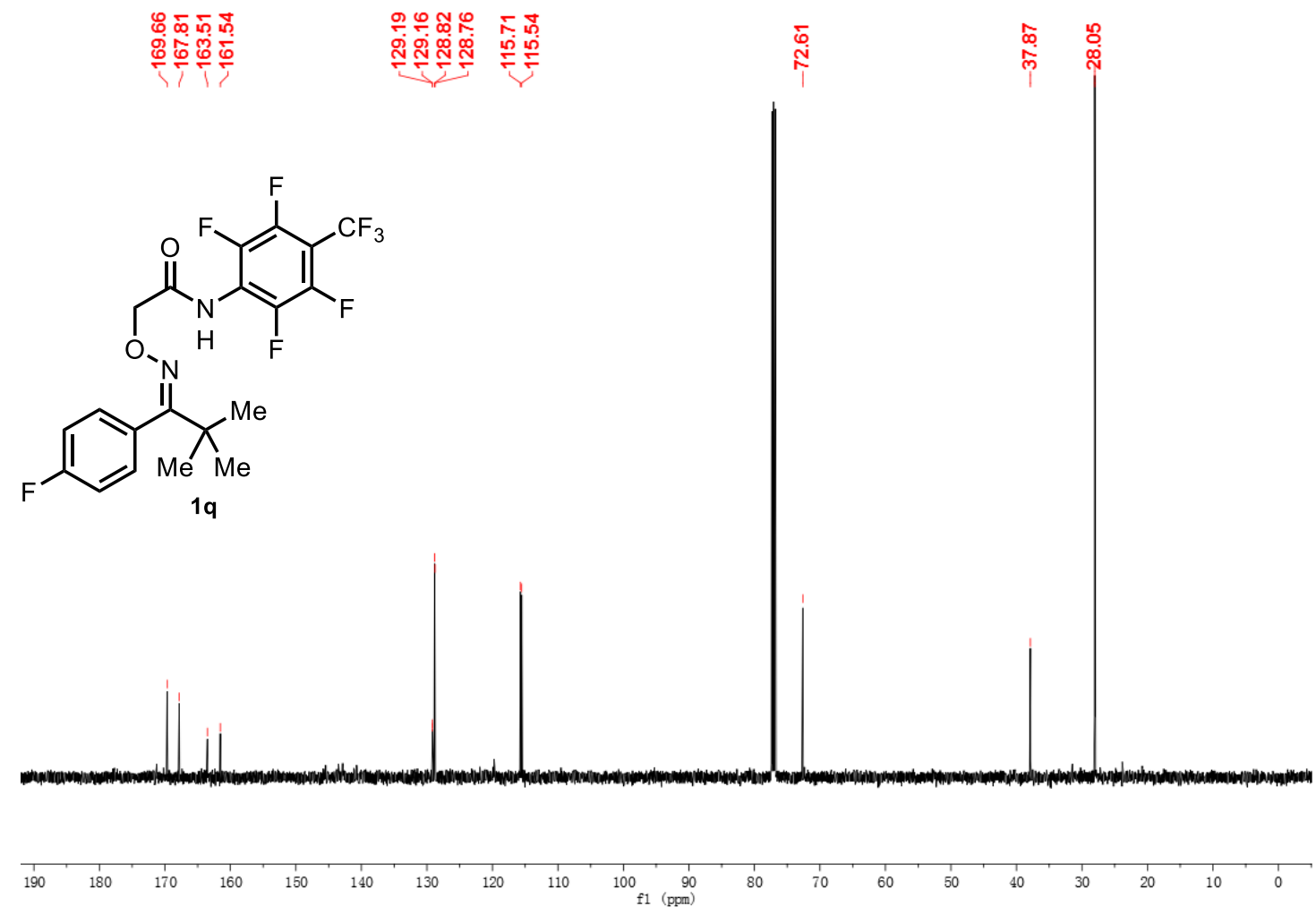


${ }^{1} \mathrm{H}$ NMR (500 MHz, $\mathrm{CDCl}_{3}$ ) spectra for $1 \mathrm{r}$

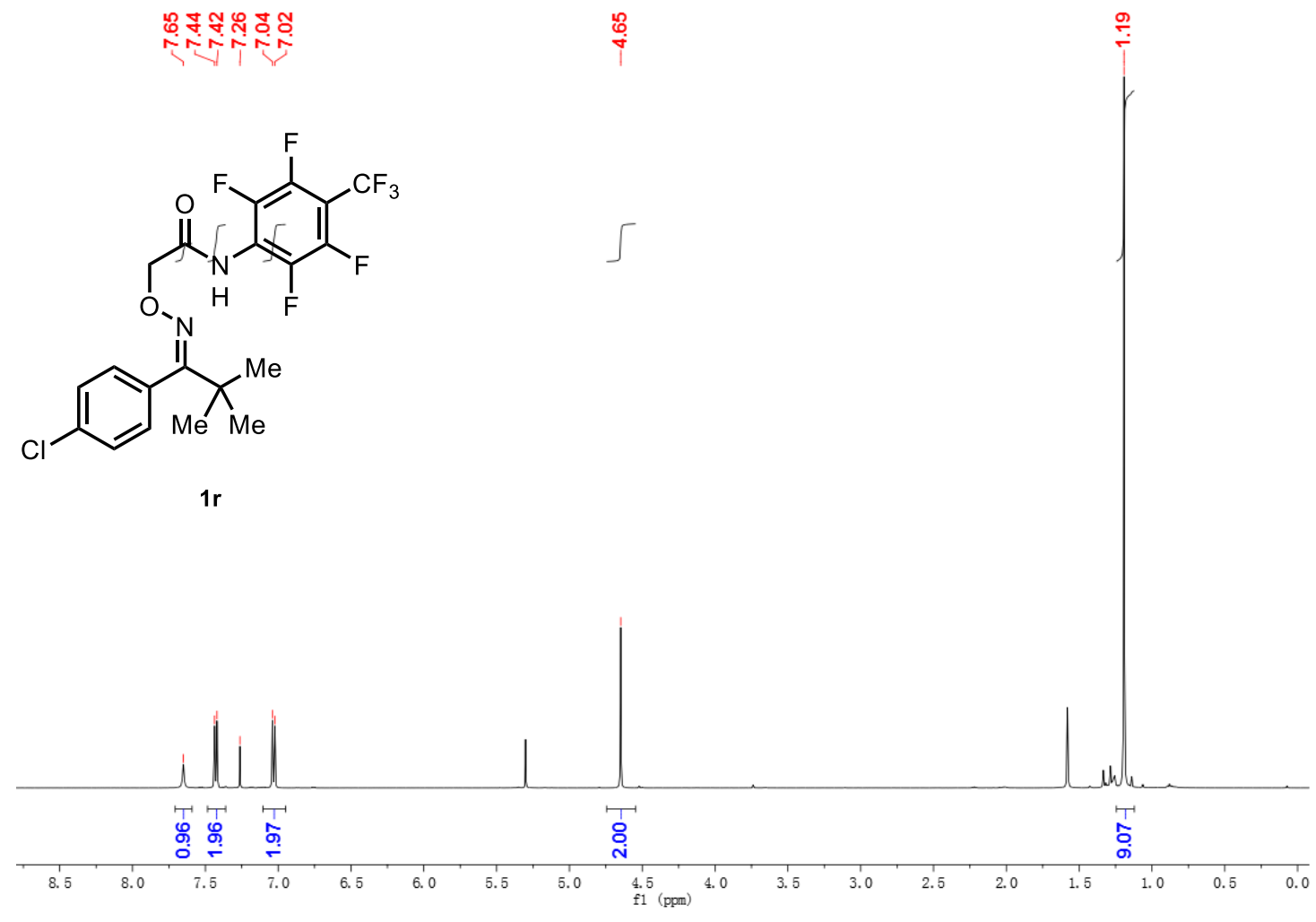

${ }^{13} \mathrm{C}$ NMR (126 MHz, $\mathrm{CDCl}_{3}$ ) spectra for $1 \mathrm{r}$
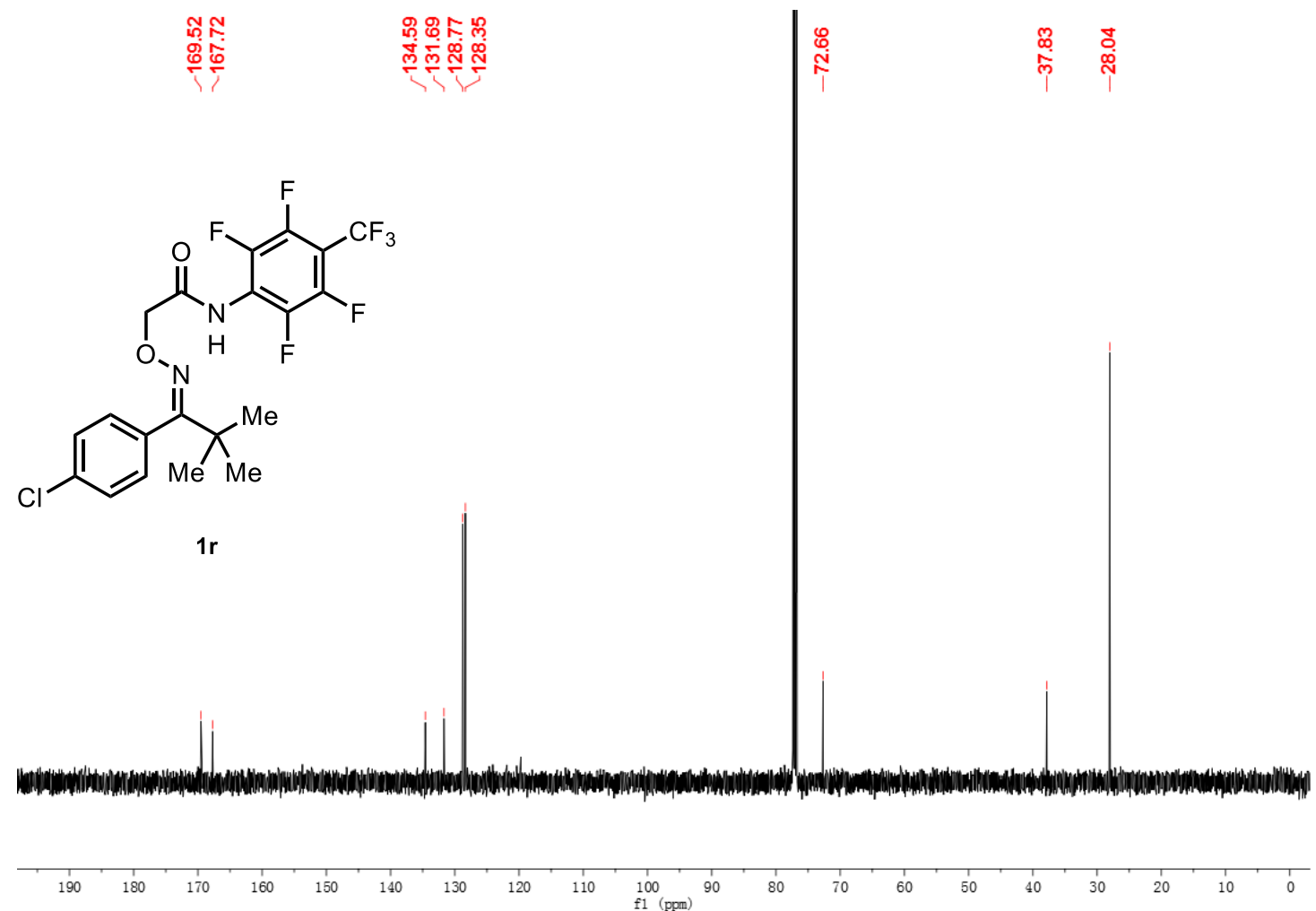
${ }^{1} \mathrm{H}$ NMR (500 MHz, CDCl $)$ spectra for $1 \mathrm{~s}$

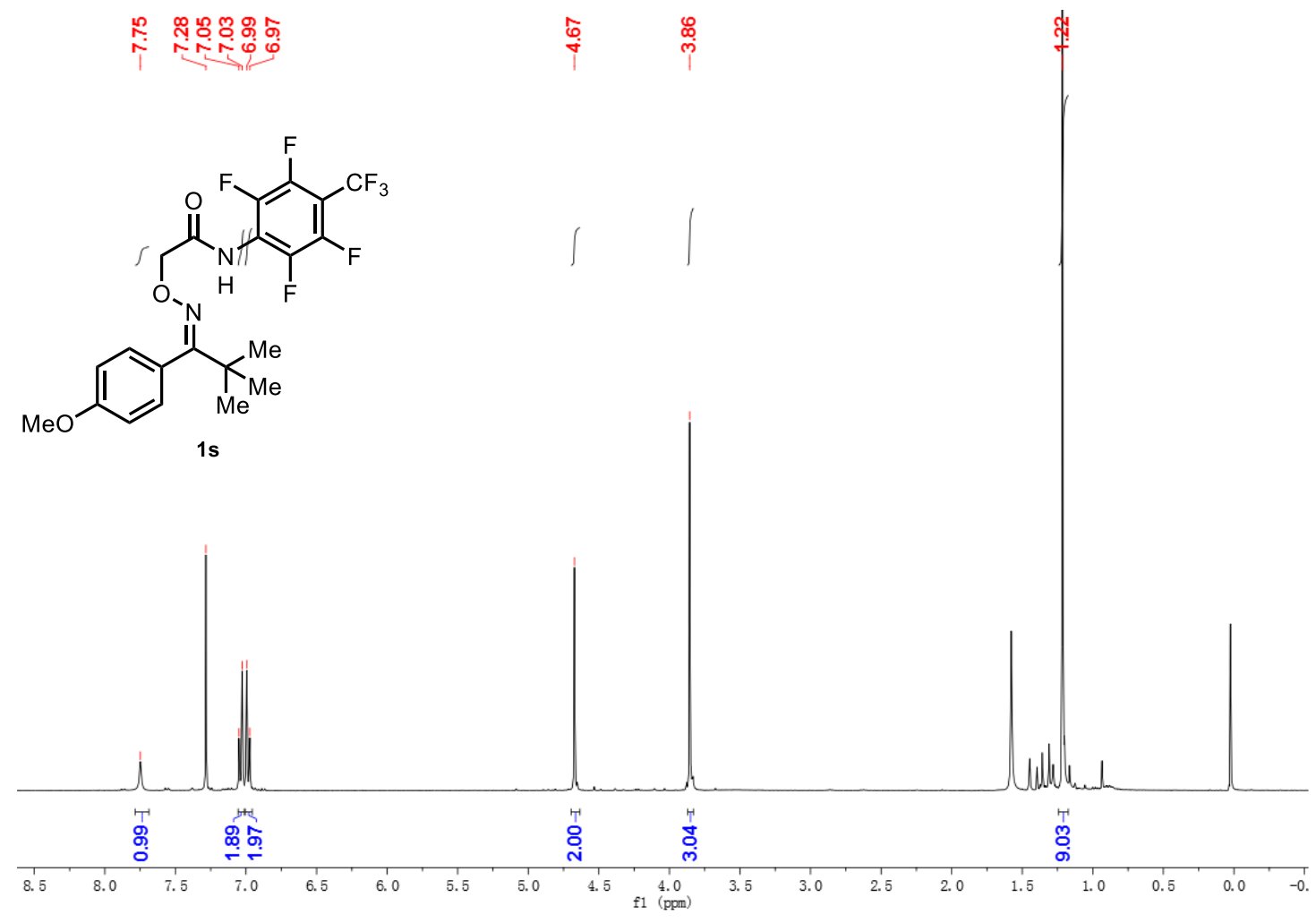

${ }^{13} \mathrm{C}$ NMR (126 MHz, $\mathrm{CDCl}_{3}$ ) spectra for $1 \mathrm{~s}$

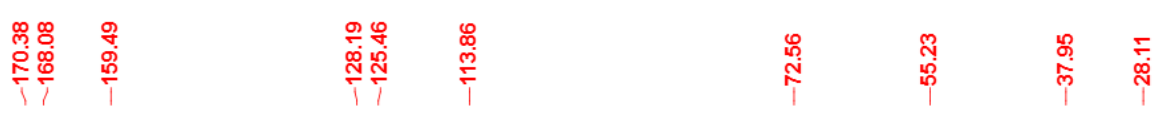
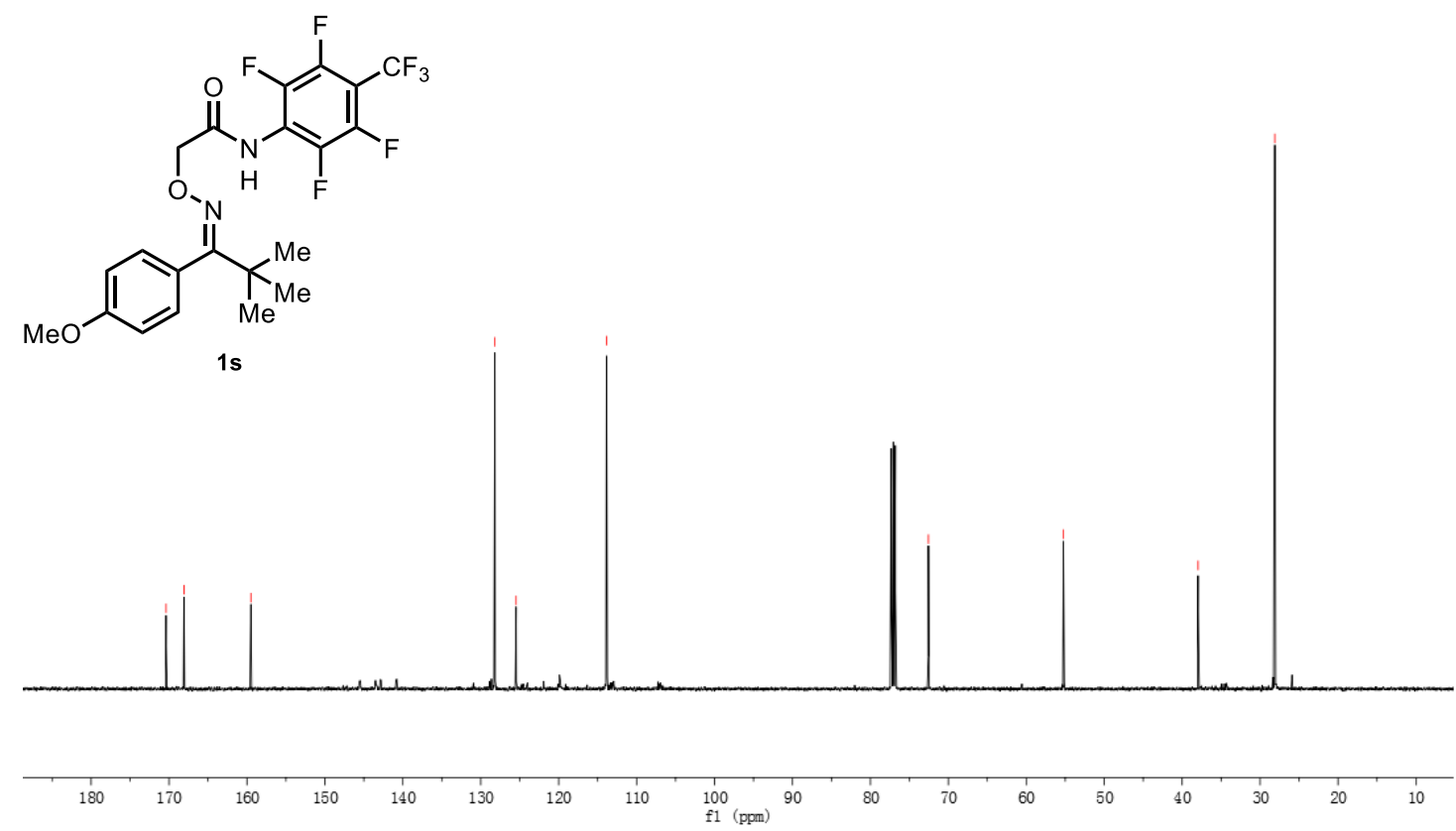
${ }^{1} \mathrm{H}$ NMR (500 MHz, $\mathrm{CDCl}_{3}$ ) spectra for $1 \mathrm{t}$

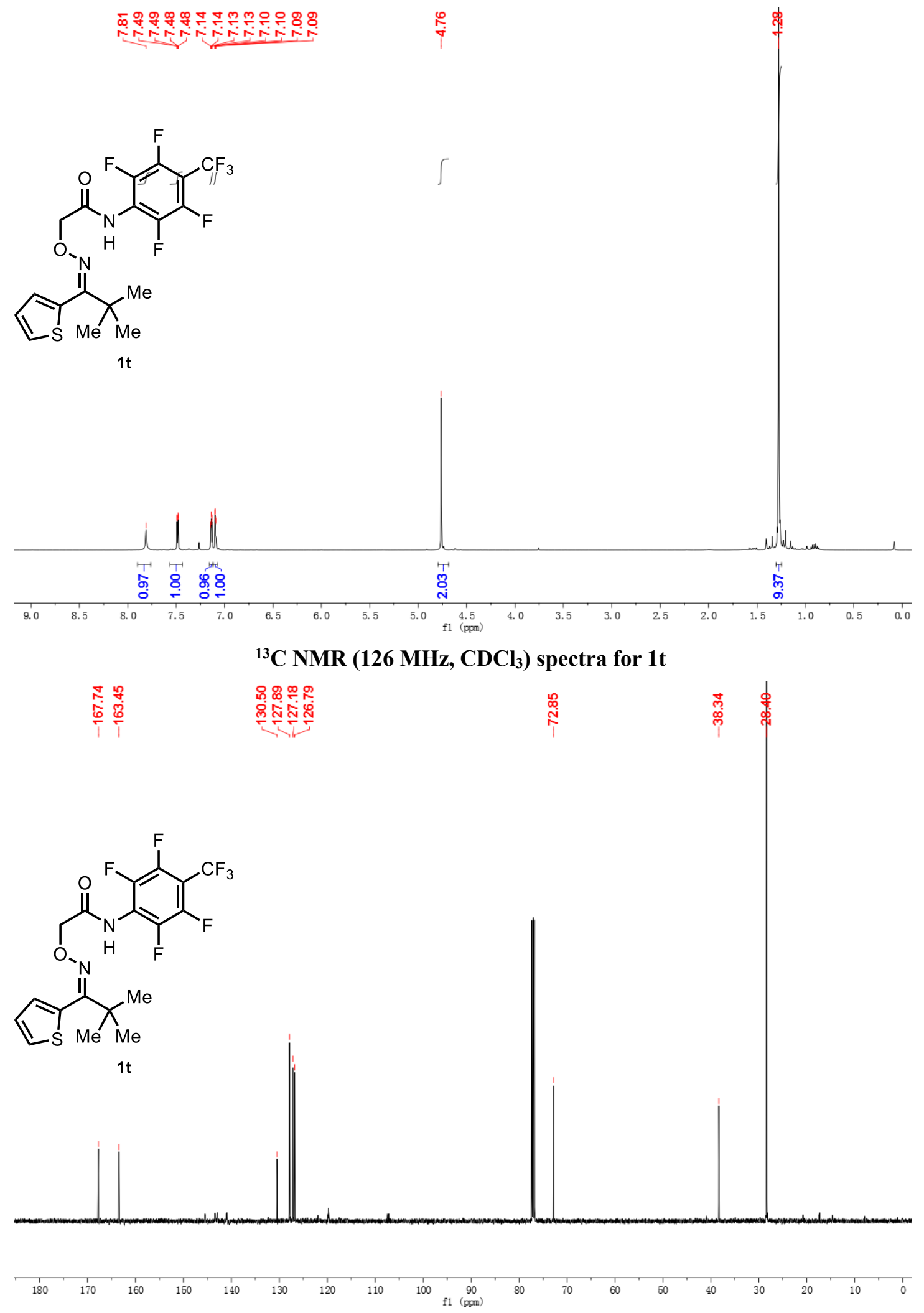


${ }^{1} \mathrm{H}$ NMR (500 MHz, $\mathrm{CDCl}_{3}$ ) spectra for $1 \mathrm{u}$

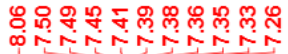

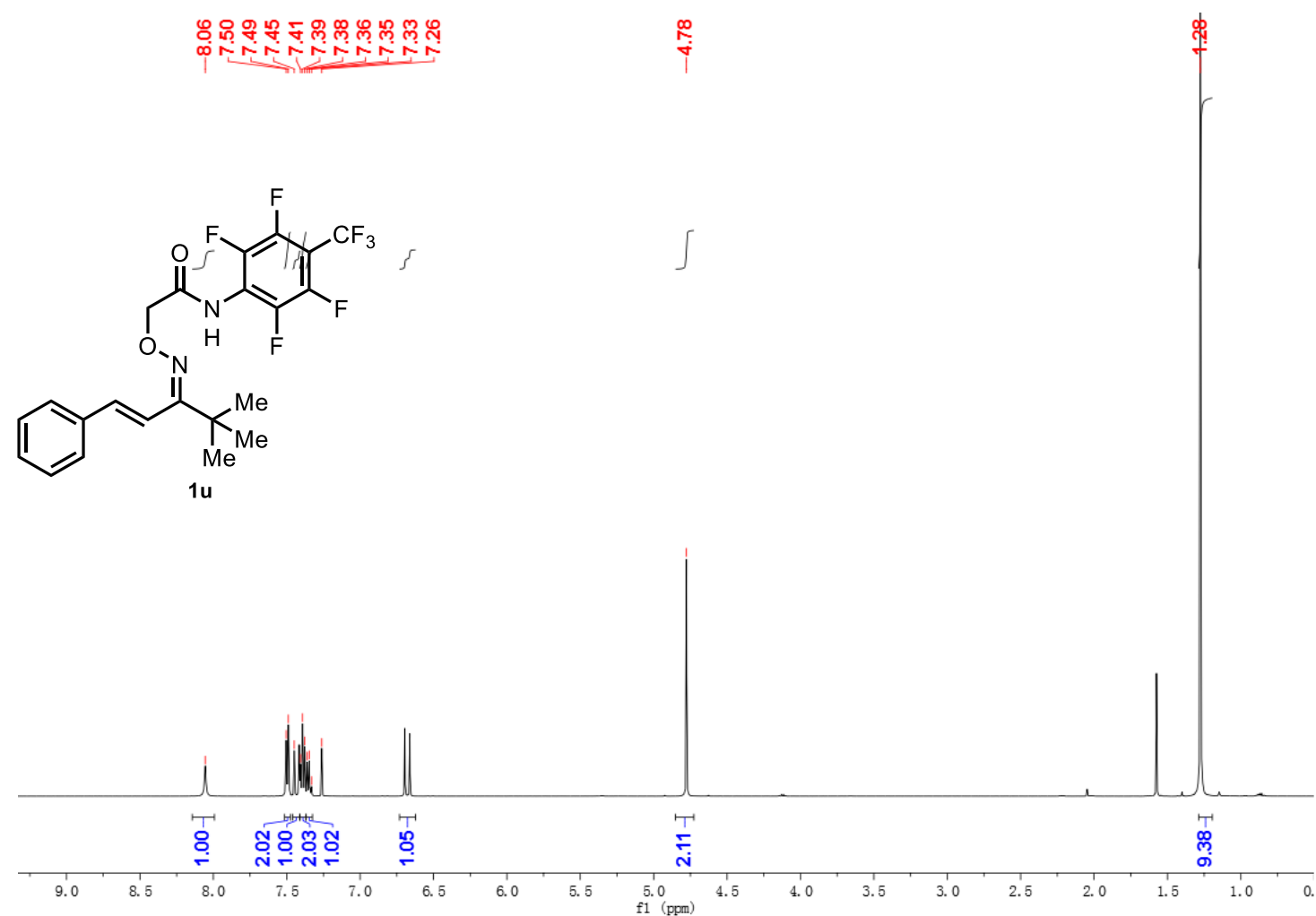

${ }^{13} \mathrm{C}$ NMR (126 MHz, $\mathrm{CDCl}_{3}$ ) spectra for $1 \mathrm{u}$

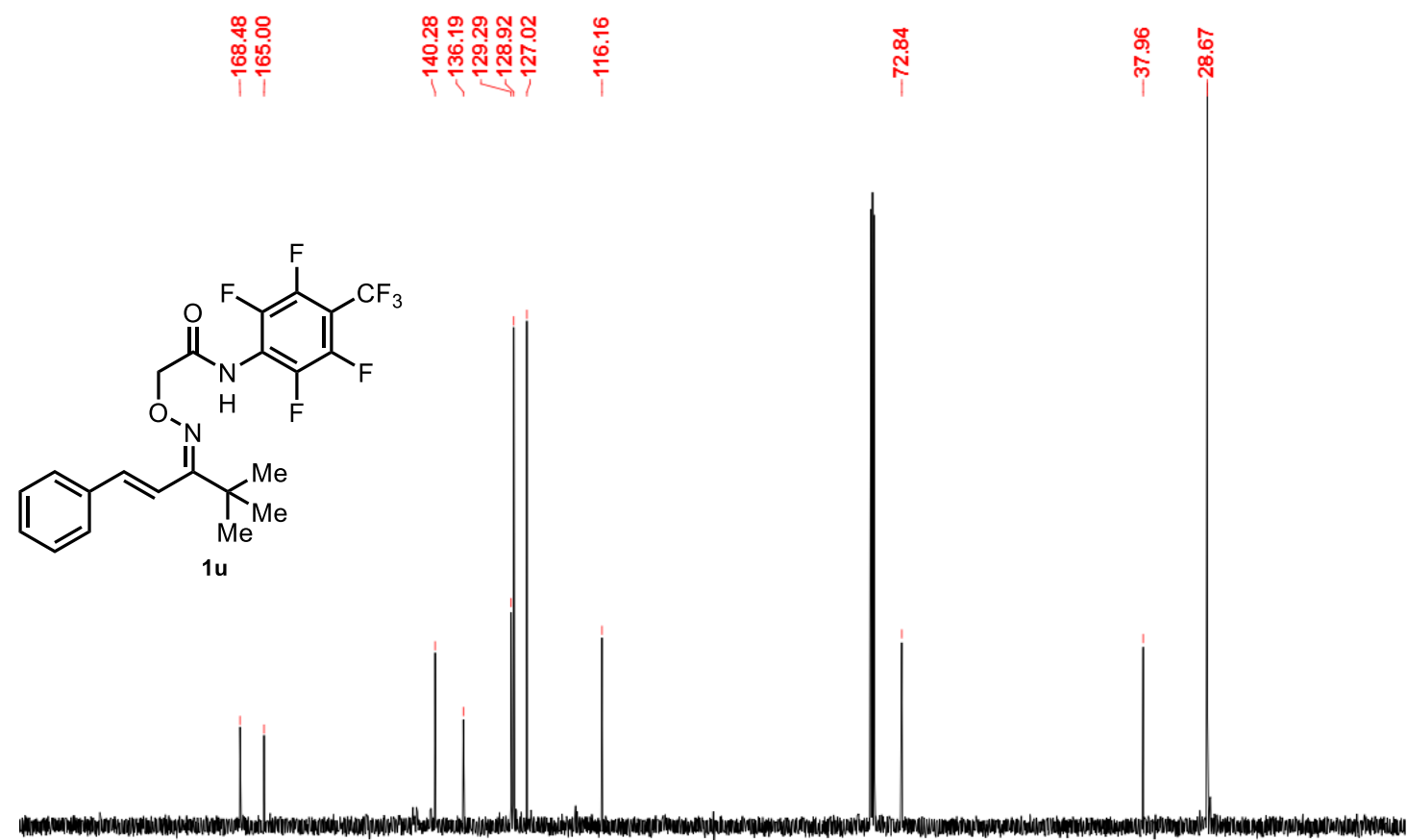

\begin{tabular}{lllllllllllllllllllllllll}
\hline & 190 & 180 & 170 & 160 & 150 & 140 & 130 & 120 & 110 & 100 & 90 & 80 & 70 & 60 & 50 & 40 & 30 & 20 & 10
\end{tabular} 
${ }^{1} \mathrm{H}$ NMR (500 MHz, $\mathrm{CDCl}_{3}$ ) spectra for $1 \mathrm{v}$

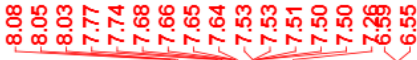

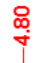<smiles>CN=C(/C=C/c1ccccc1[N+](=O)[O-])C(C)(C)C</smiles><smiles>NOCC(=O)Nc1c(F)c(F)c(C(F)(F)F)c(F)c1F</smiles>

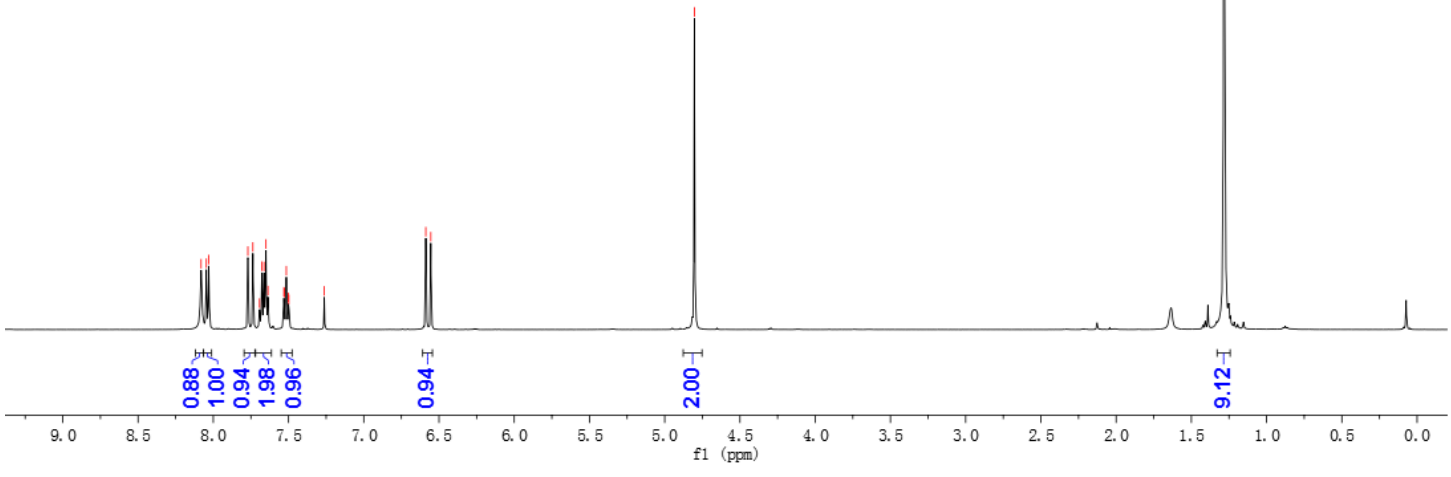

${ }^{13} \mathrm{C}$ NMR (126 MHz, $\mathrm{CDCl}_{3}$ ) spectra for $1 \mathrm{v}$

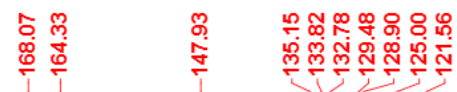<smiles>CC(C)(C)C(/C=C/c1ccccc1[N+](=O)[O-])=N/O</smiles><smiles>NOCC(=O)Nc1c(F)c(F)c(C(F)(F)F)c(F)c1F</smiles>
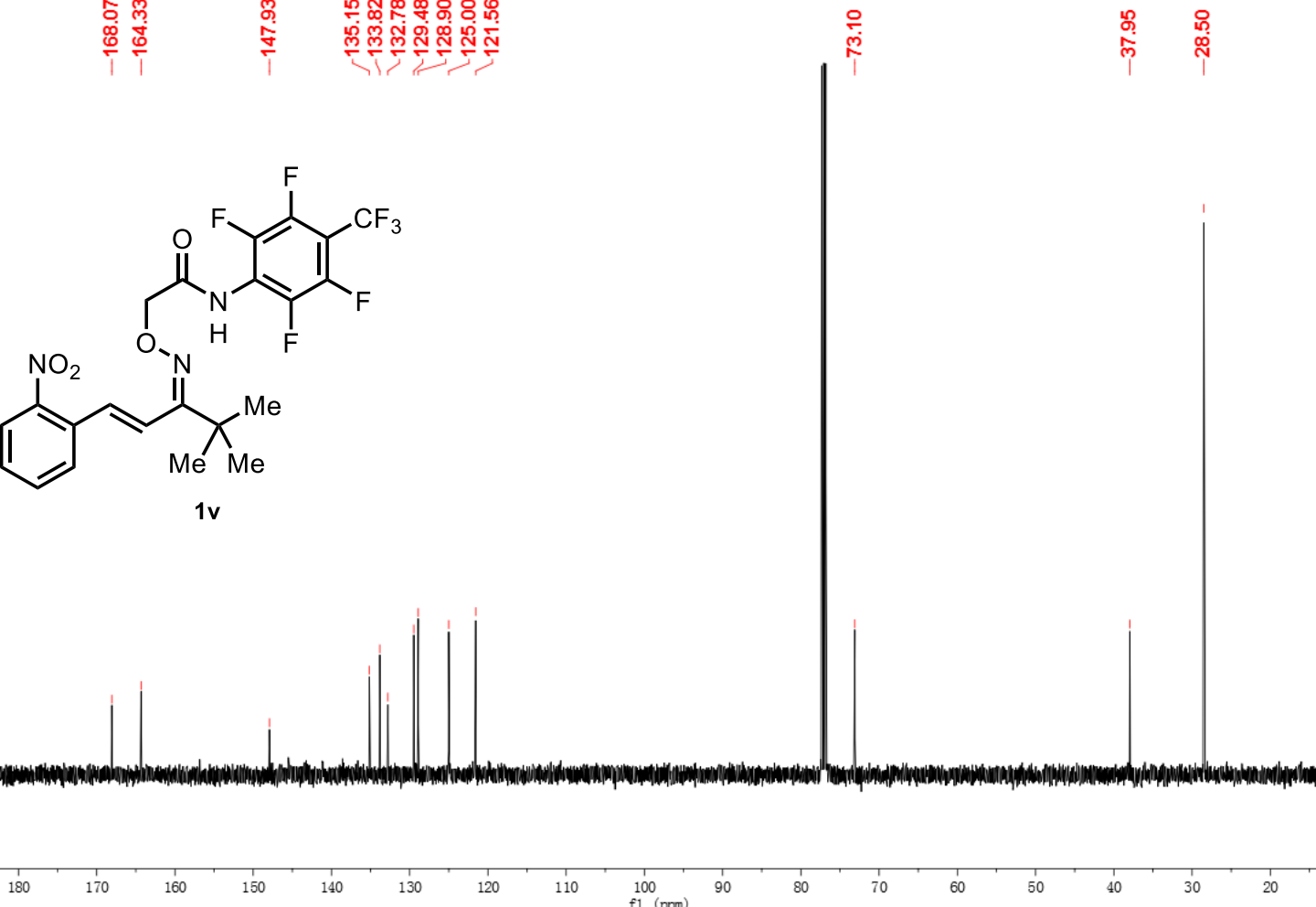

$\begin{array}{rrrr}140 & 130 & 120 & 110\end{array}$ f1 (ppm) 
${ }^{1} \mathrm{H}$ NMR (500 MHz, $\mathrm{CDCl}_{3}$ ) spectra for $1 \mathrm{w}$

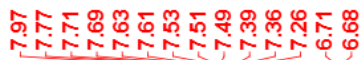

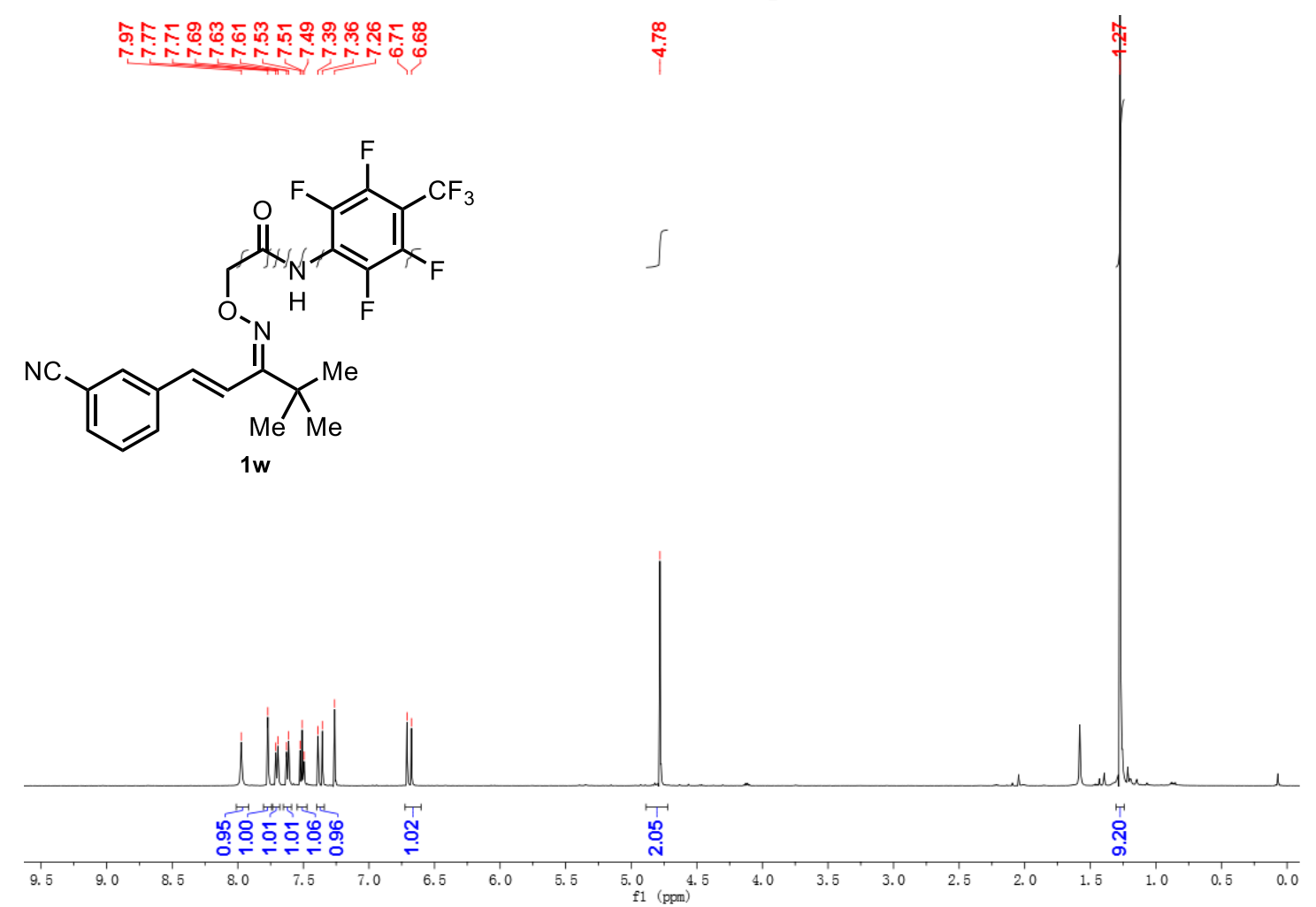

${ }^{13} \mathrm{C}$ NMR (126 MHz, $\mathrm{CDCl}_{3}$ ) spectra for $1 \mathrm{w}$

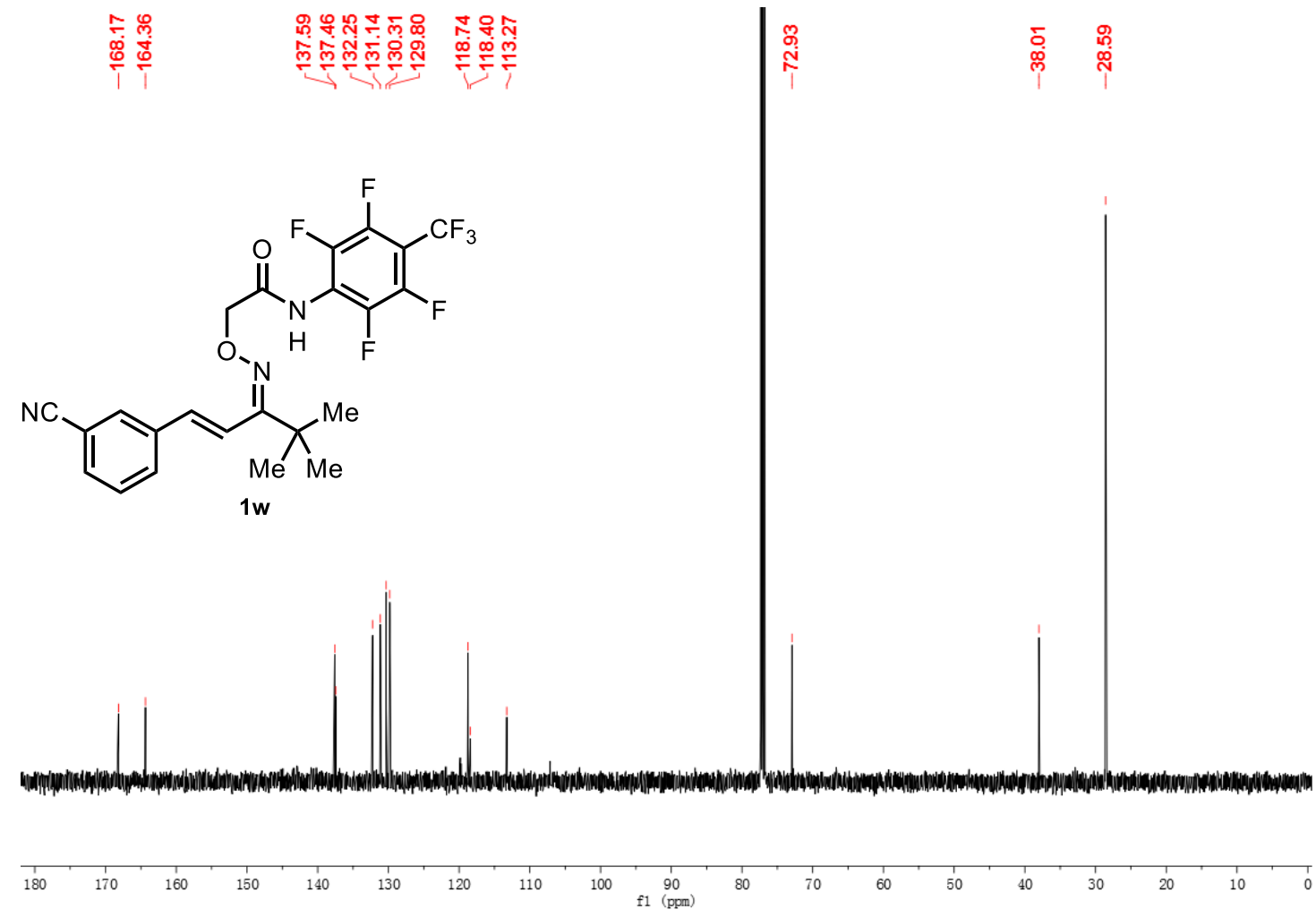


${ }^{1} \mathrm{H}$ NMR (500 MHz, $\mathrm{CDCl}_{3}$ ) spectra for $1 \mathrm{x}$

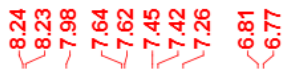

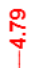<smiles>CCO/N=C(\C=C\c1ccc([N+](=O)[O-])cc1)C(C)(C)C</smiles>

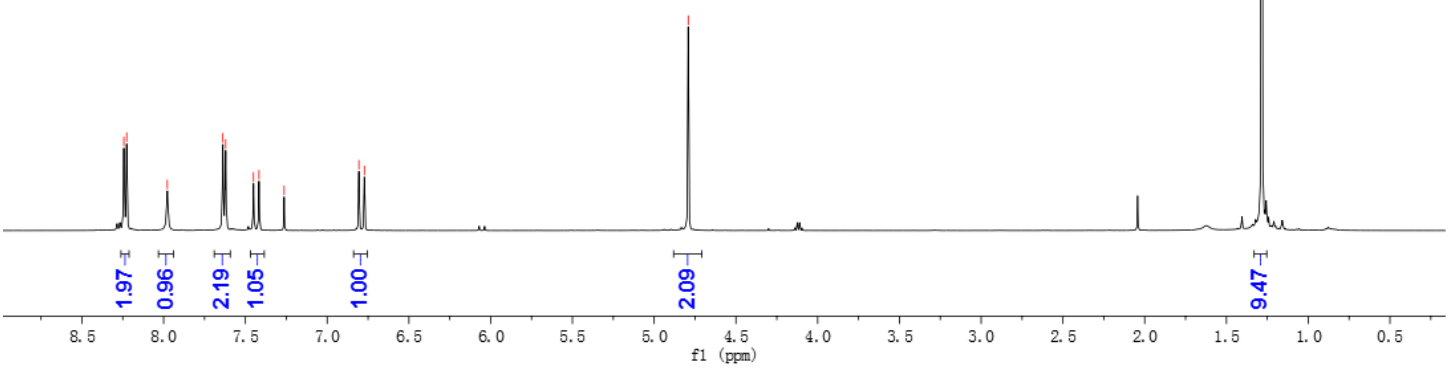

${ }^{13} \mathrm{C}$ NMR (126 MHz, $\mathrm{CDCl}_{3}$ ) spectra for $1 \mathrm{x}$

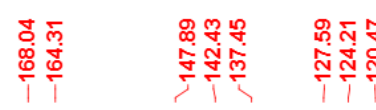

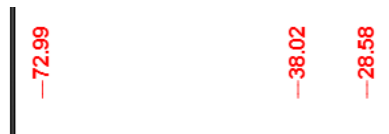<smiles>C/C=C(F)/C(=C/C)NC(=O)CO/N=C(\C=C\c1ccc([N+](=O)[O-])cc1)C(C)(C)C</smiles>
$1 \mathrm{x}$

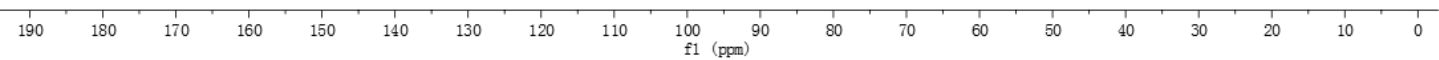


${ }^{1} \mathrm{H}$ NMR (500 MHz, $\mathrm{CDCl}_{3}$ ) spectra for $1 \mathrm{y}$<smiles>CC(C)(C)C(/C=C/c1ccc(F)cc1)=N\OCC(=O)Nc1c(F)c(F)c(C(F)(F)F)c(F)c1F</smiles>

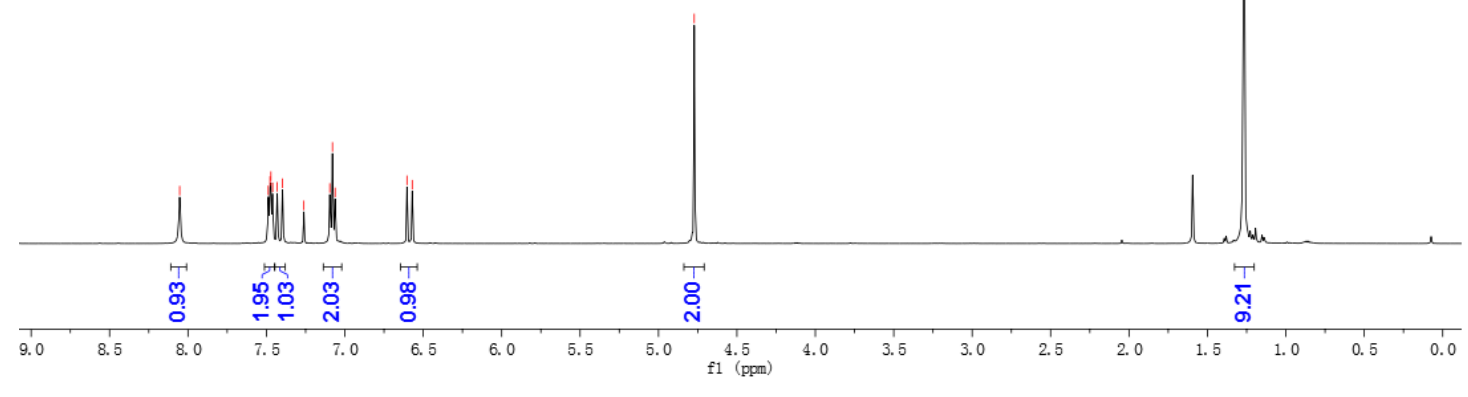

${ }^{13} \mathrm{C}$ NMR (126 MHz, $\mathrm{CDCl}_{3}$ ) spectra for $1 \mathrm{y}$

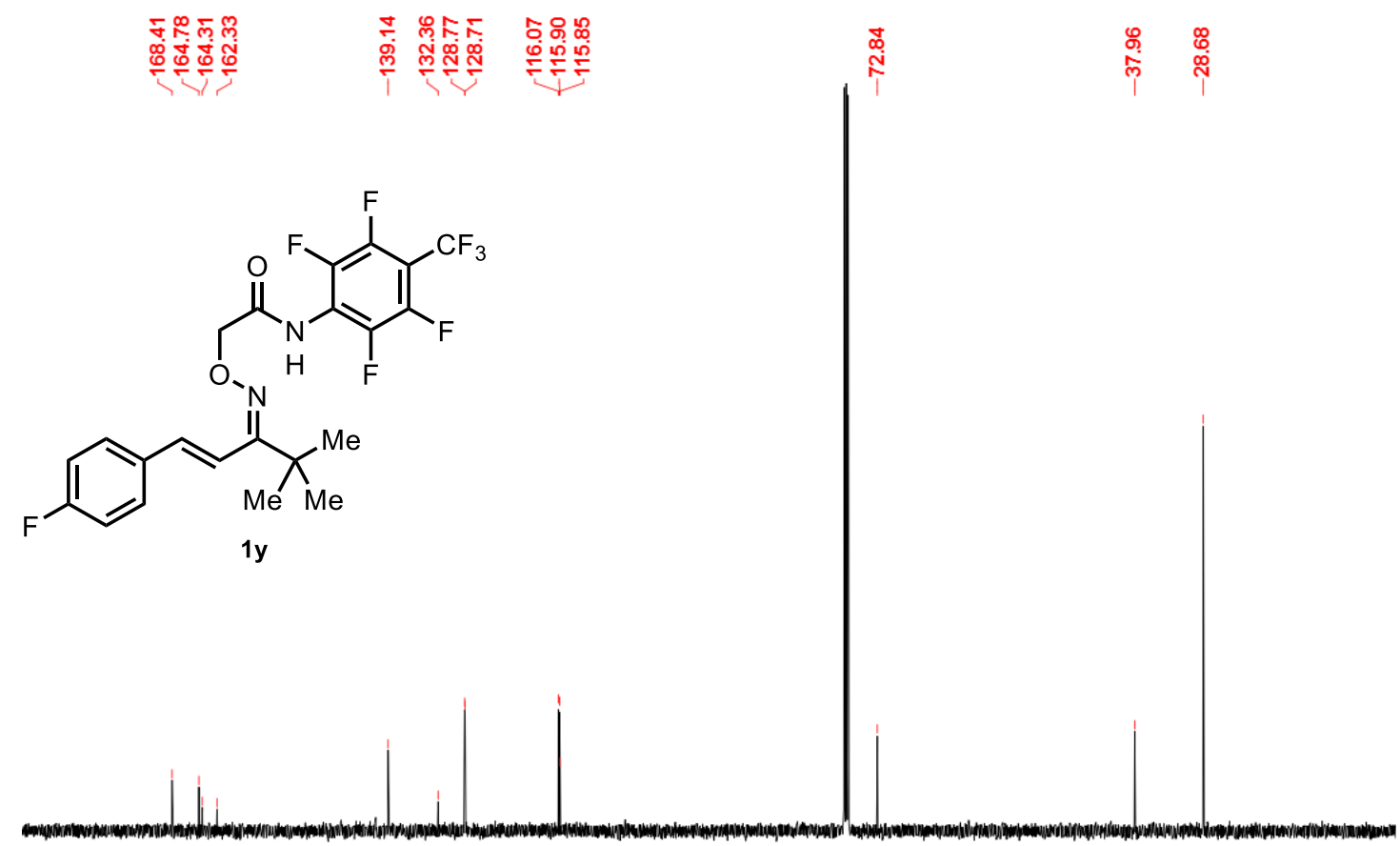

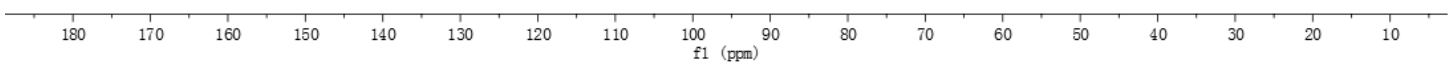


${ }^{1} \mathrm{H}$ NMR (500 MHz, $\left.\mathrm{CDCl}_{3}\right)$ spectra for $1 z$

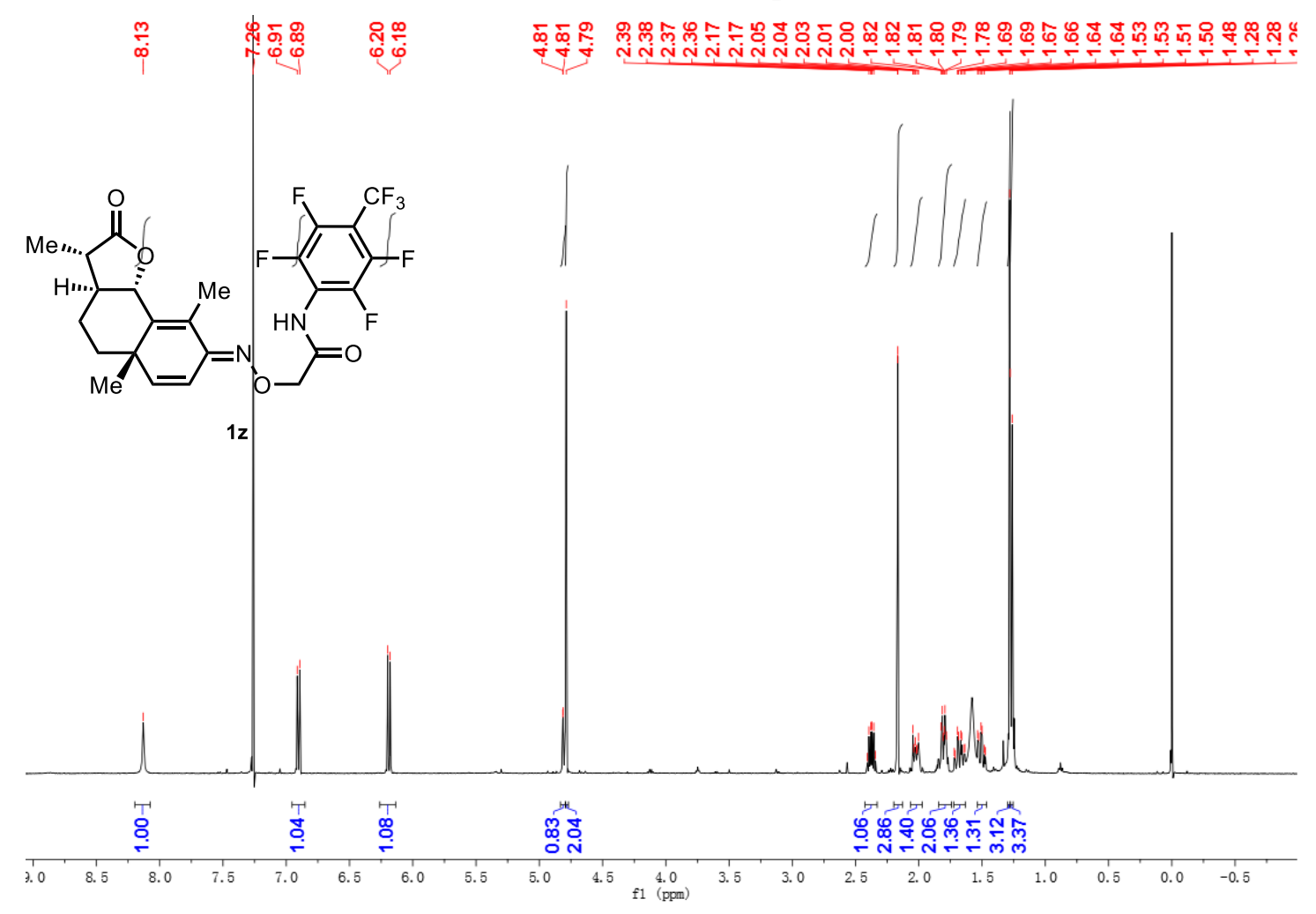

${ }^{13} \mathrm{C}$ NMR (126 MHz, $\left.\mathrm{CDCl}_{3}\right)$ spectra for $1 \mathrm{z}$

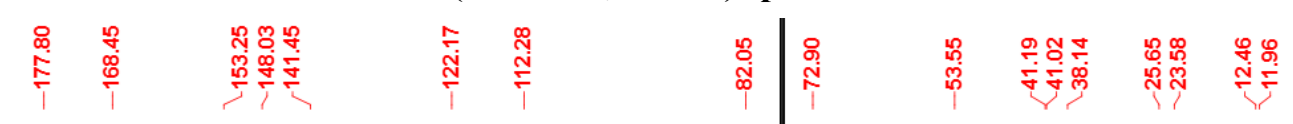<smiles>CC1=C2[C@@H]3OC(=O)[C@H](C)[C@@H]3CC[C@]2(C)C=C/C1=N\OCC(=O)Nc1c(F)c(F)c(C(F)(F)F)c(F)c1F</smiles>

$1 z$
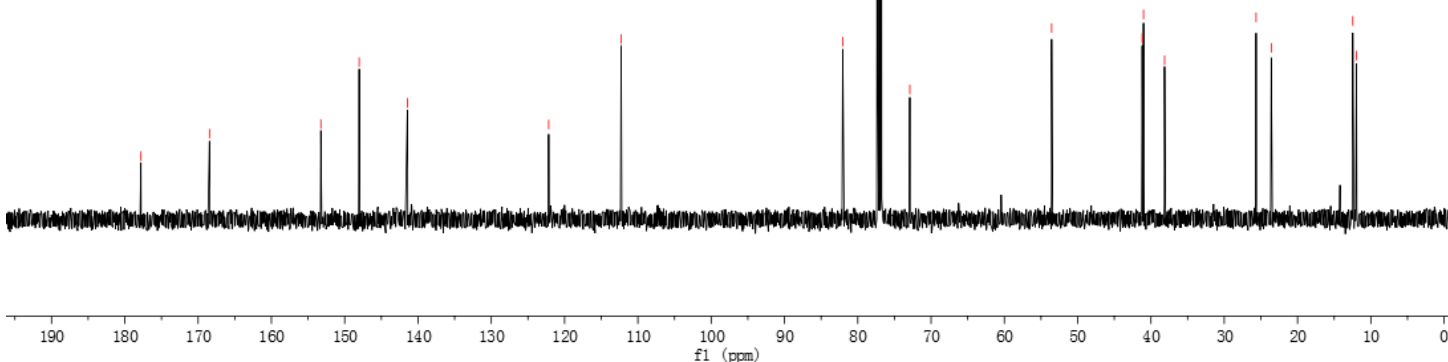
${ }^{1} \mathrm{H}$ NMR (500 MHz, $\mathrm{CDCl}_{3}$ ) spectra for $2 \mathrm{a}$

$\stackrel{m}{\infty} \stackrel{\substack{0 \\ i}}{T}$

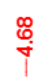

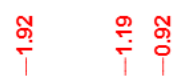

CN

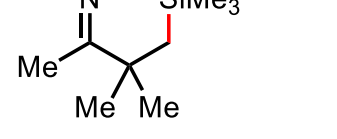

$2 a$

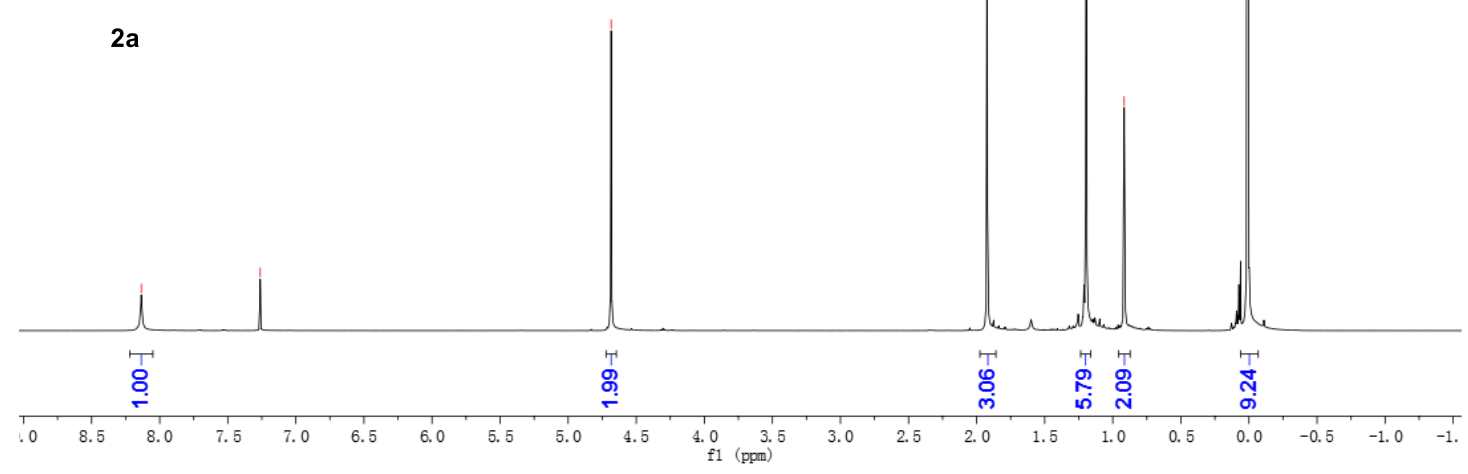

${ }^{13} \mathrm{C}$ NMR (126 MHz, $\mathrm{CDCl}_{3}$ ) spectra for $2 \mathrm{a}$<smiles>CCC(C)(C(C)=O)/C(C)=N\OCC(=O)Nc1c(F)c(F)c(C(F)(F)F)c(F)c1F</smiles>

$2 a$

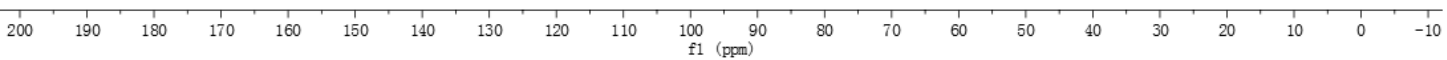


${ }^{1} \mathrm{H}$ NMR (500 MHz, $\left.\mathrm{CDCl}_{3}\right)$ spectra for $2 \mathrm{~b}$

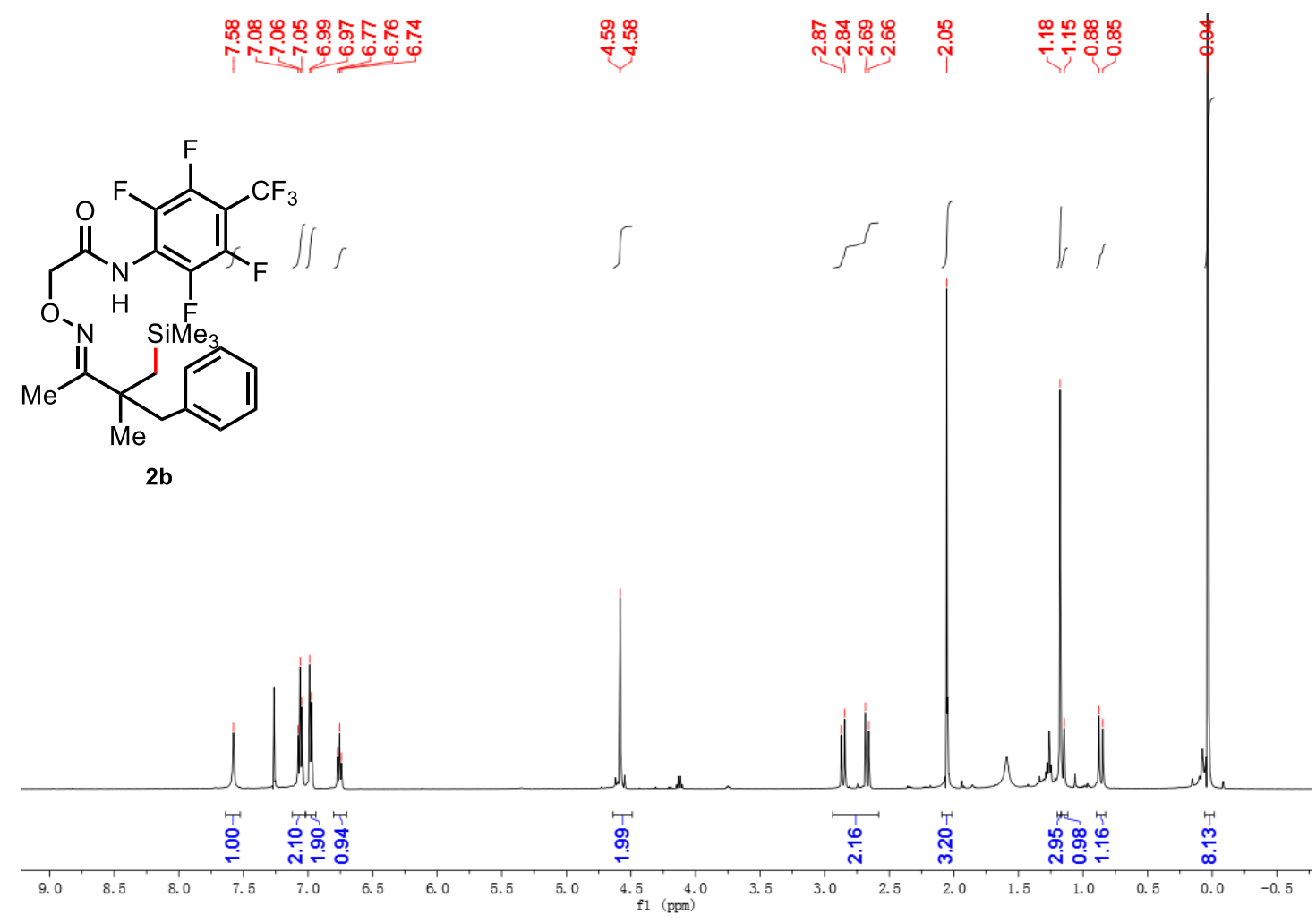

${ }^{13} \mathrm{C}$ NMR (126 MHz, $\left.\mathrm{CDCl}_{3}\right)$ spectra for $2 \mathrm{~b}$

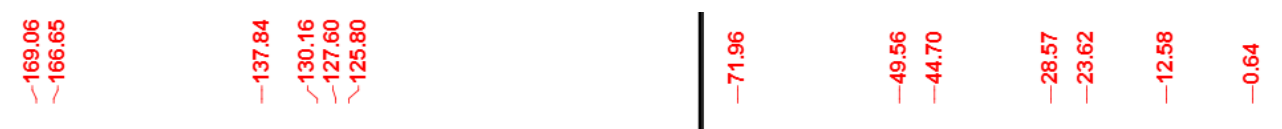<smiles>C/C(=N\OCC(=O)Nc1c(F)c(F)c(C(F)(F)F)c(F)c1F)C(C)(CS)Cc1ccccc1</smiles>

2b

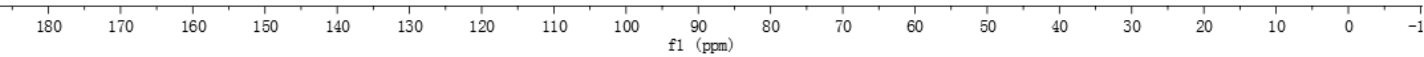


${ }^{1} \mathrm{H}$ NMR (500 MHz, $\left.\mathrm{CDCl}_{3}\right)$ spectra for $2 \mathrm{c}$

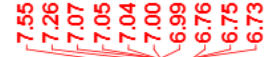

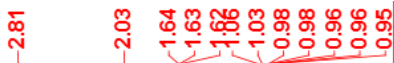<smiles>CCC(CC)(Cc1ccccc1)/C(C)=N/OCC(=O)Nc1c(F)c(F)c(C(F)(F)F)c(F)c1F</smiles>

2c

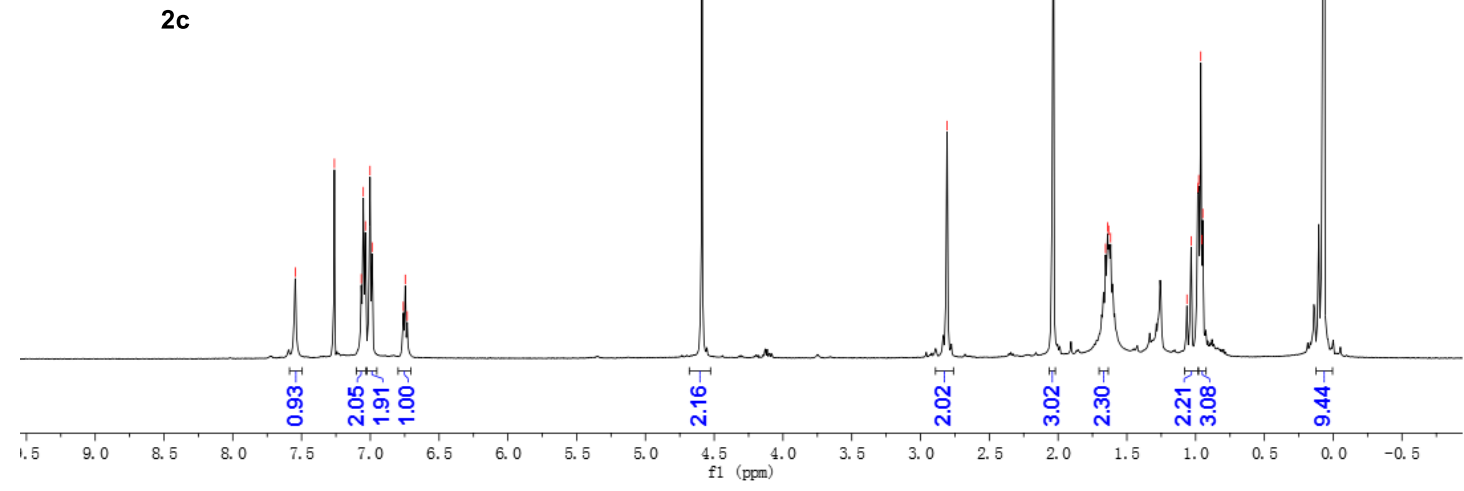

${ }^{13} \mathrm{C}$ NMR (126 MHz, $\left.\mathrm{CDCl}_{3}\right)$ spectra for 2c

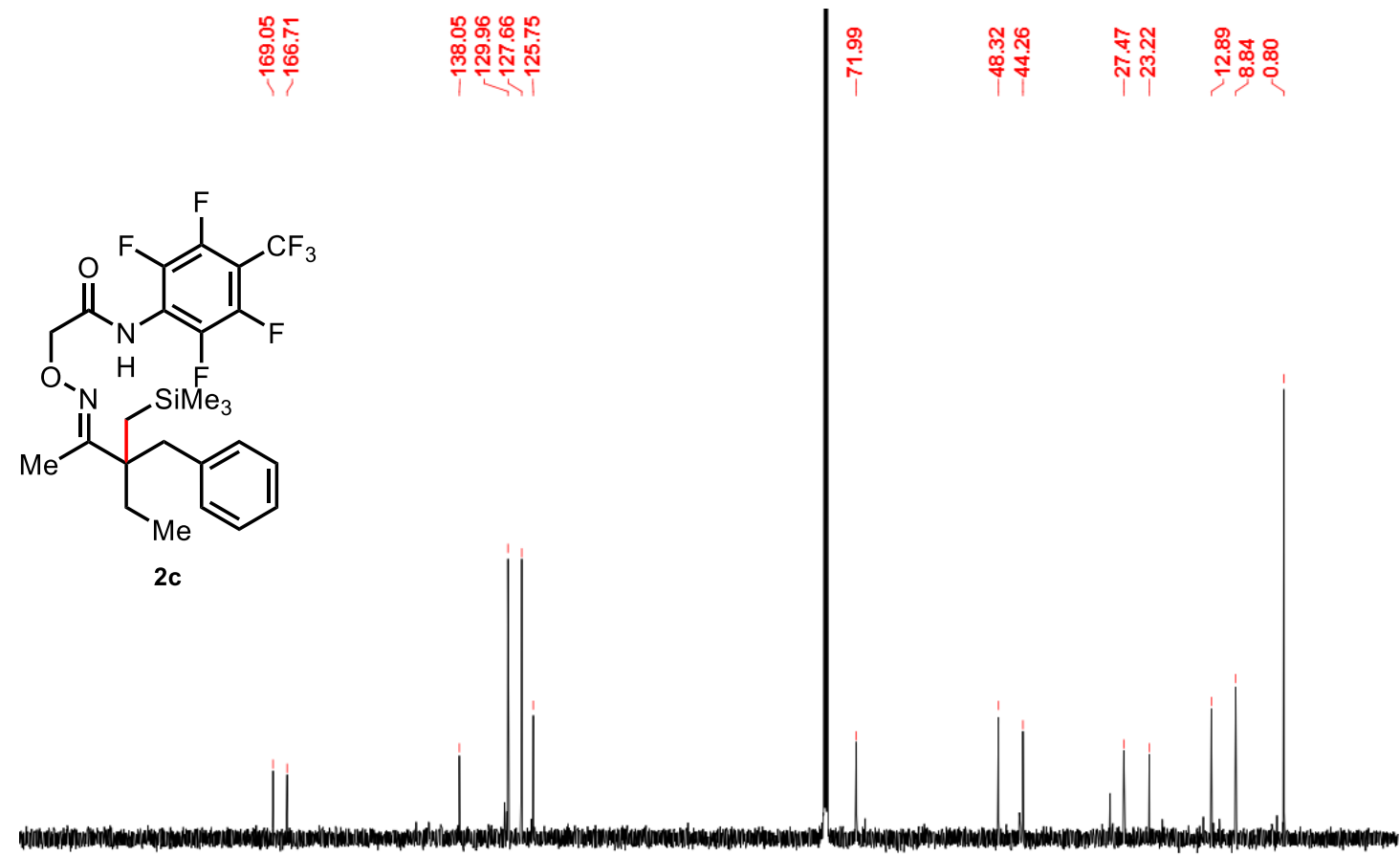

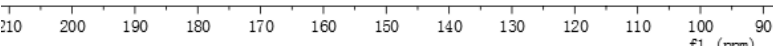


${ }^{1}$ H NMR (500 MHz, $\left.\mathrm{CDCl}_{3}\right)$ spectra for 2d

둥

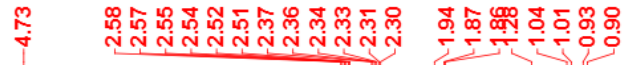<smiles>C=Cc1c(F)c(NC(=O)CON)c(F)c(F)c1C(F)(F)F</smiles>
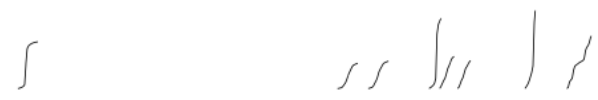<smiles>CCC(C)(CCc1ccccc1)C(C)=N</smiles>

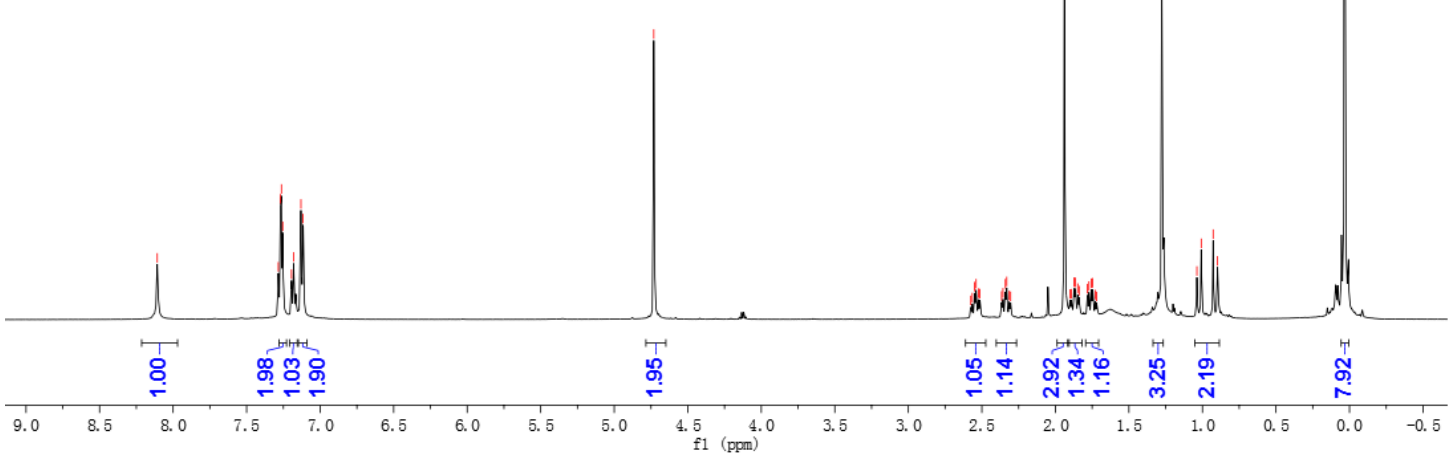

${ }^{13} \mathrm{C}$ NMR (126 MHz, $\left.\mathrm{CDCl}_{3}\right)$ spectra for 2d

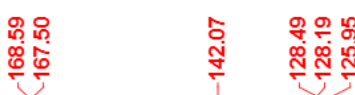

每

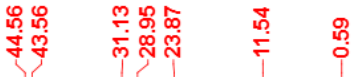<smiles>CS(C)(C)(C)(C)c1c(F)c(C(F)(F)F)c(F)c(F)c1NC(=O)CON</smiles><smiles>COCC(C)(CCc1ccccc1)C(C)=N</smiles>

2d

$\begin{array}{llllllllll}180 & 170 & 160 & 150 & 140 & 130 & 120 & 110 & 100 & 90 \\ f 1(\mathrm{ppm}) & 80\end{array}$ 
${ }^{1} \mathrm{H}$ NMR (500 MHz, $\left.\mathrm{CDCl}_{3}\right)$ spectra for $2 \mathrm{e}$

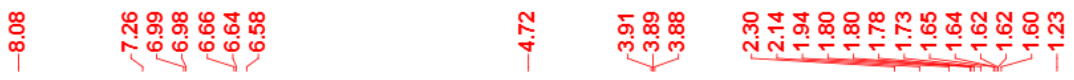<smiles>CS(C)(C)c1c(F)c(C(F)(F)F)c(F)c(F)c1NC(=O)CON</smiles><smiles>CCSCC(C)(CCCOc1cc(C)ccc1C)C(C)=NC</smiles>

$2 e$
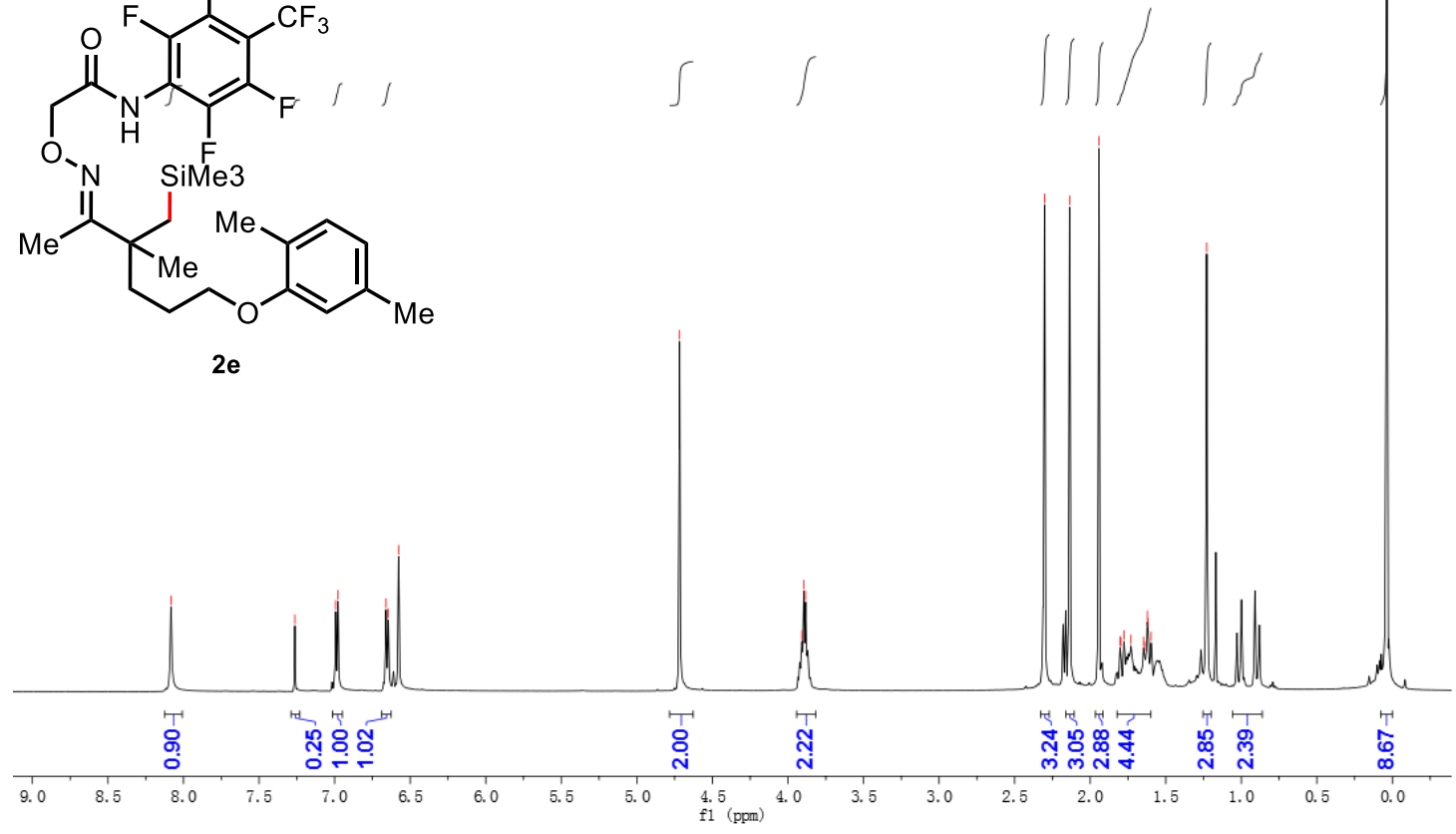

${ }^{13} \mathrm{C}$ NMR (126 MHz, $\left.\mathrm{CDCl}_{3}\right)$ spectra for $2 \mathrm{e}$

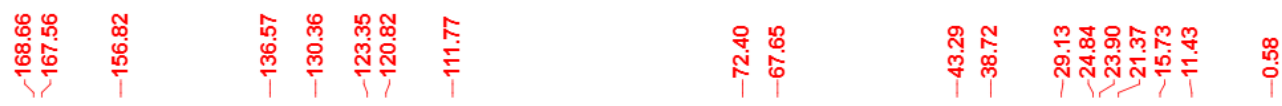<smiles></smiles><smiles>CSCCC(C)(CCCOc1cc(C)ccc1C)C(C)=NO</smiles>

$2 e$

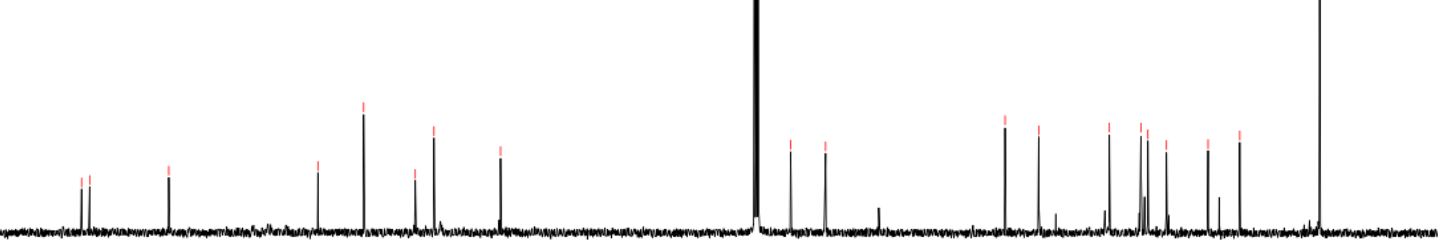

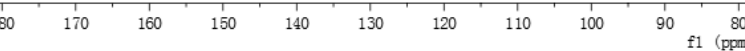


${ }^{1} \mathrm{H}$ NMR (500 MHz, $\mathrm{CDCl}_{3}$ ) spectra for $2 \mathrm{f}$

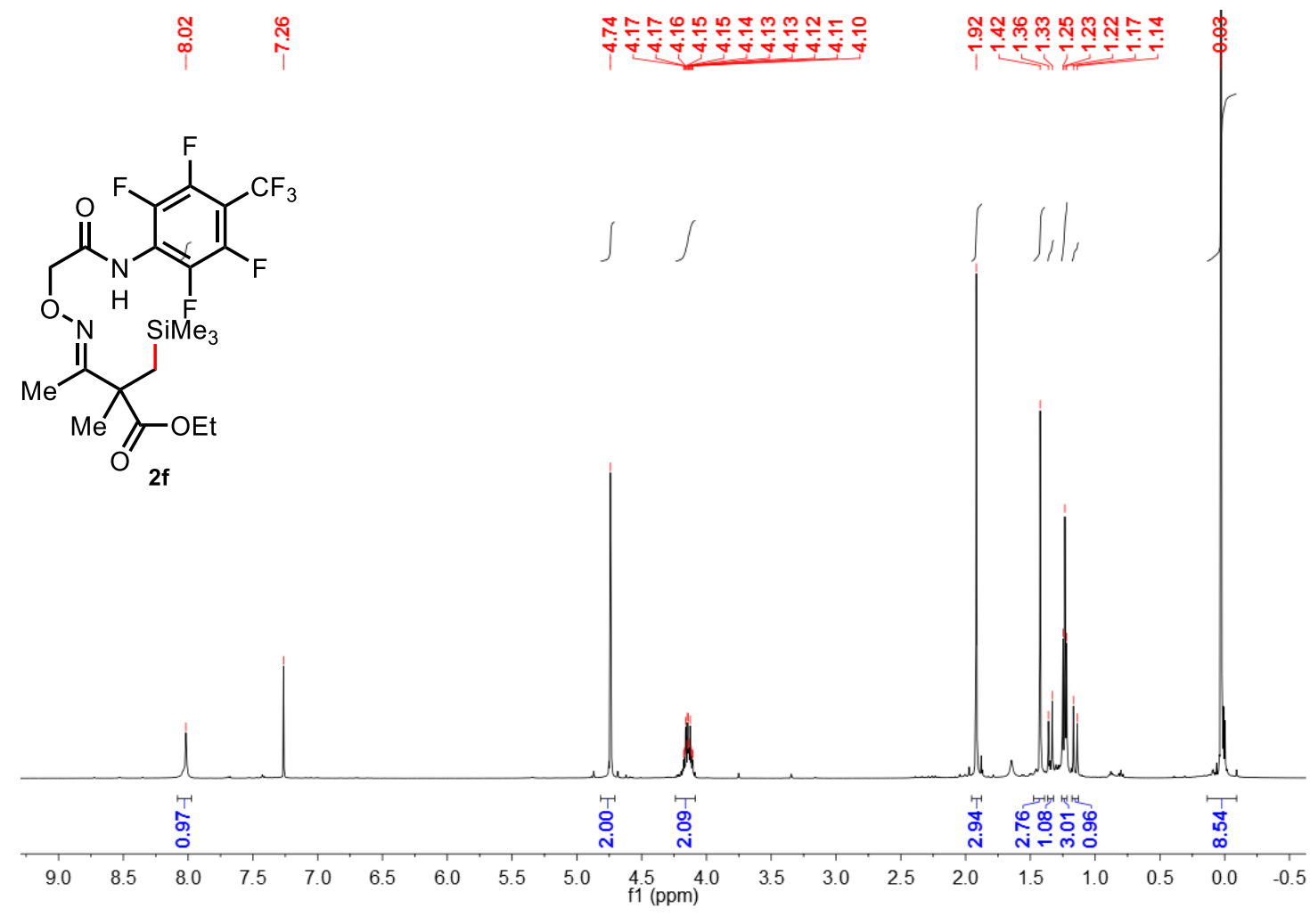

${ }^{13} \mathrm{C}$ NMR (126 MHz, $\mathrm{CDCl}_{3}$ ) spectra for $2 \mathrm{f}$
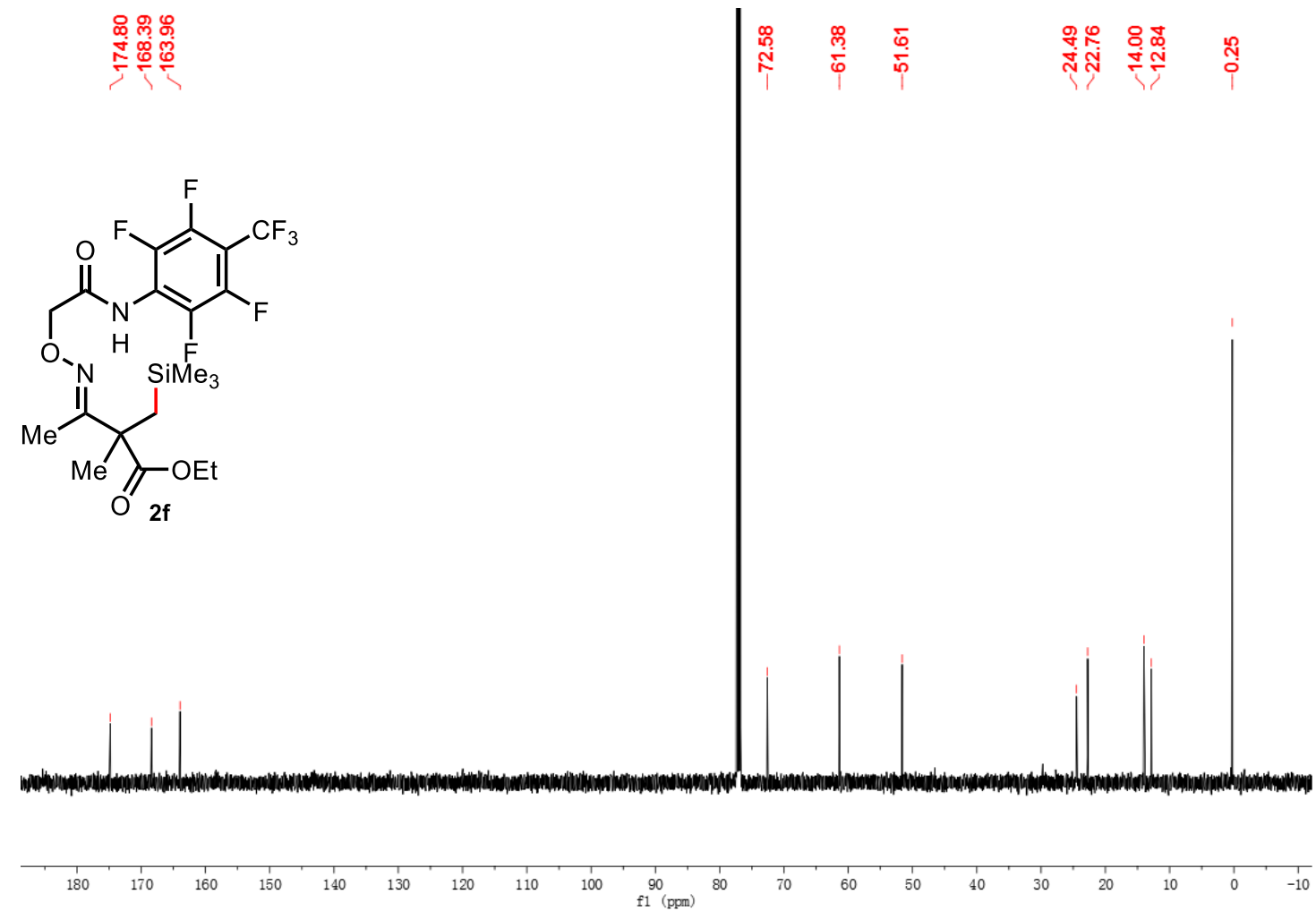
${ }^{1} \mathrm{H}$ NMR (500 MHz, $\left.\mathrm{CDCl}_{3}\right)$ spectra for $2 \mathrm{~g}$

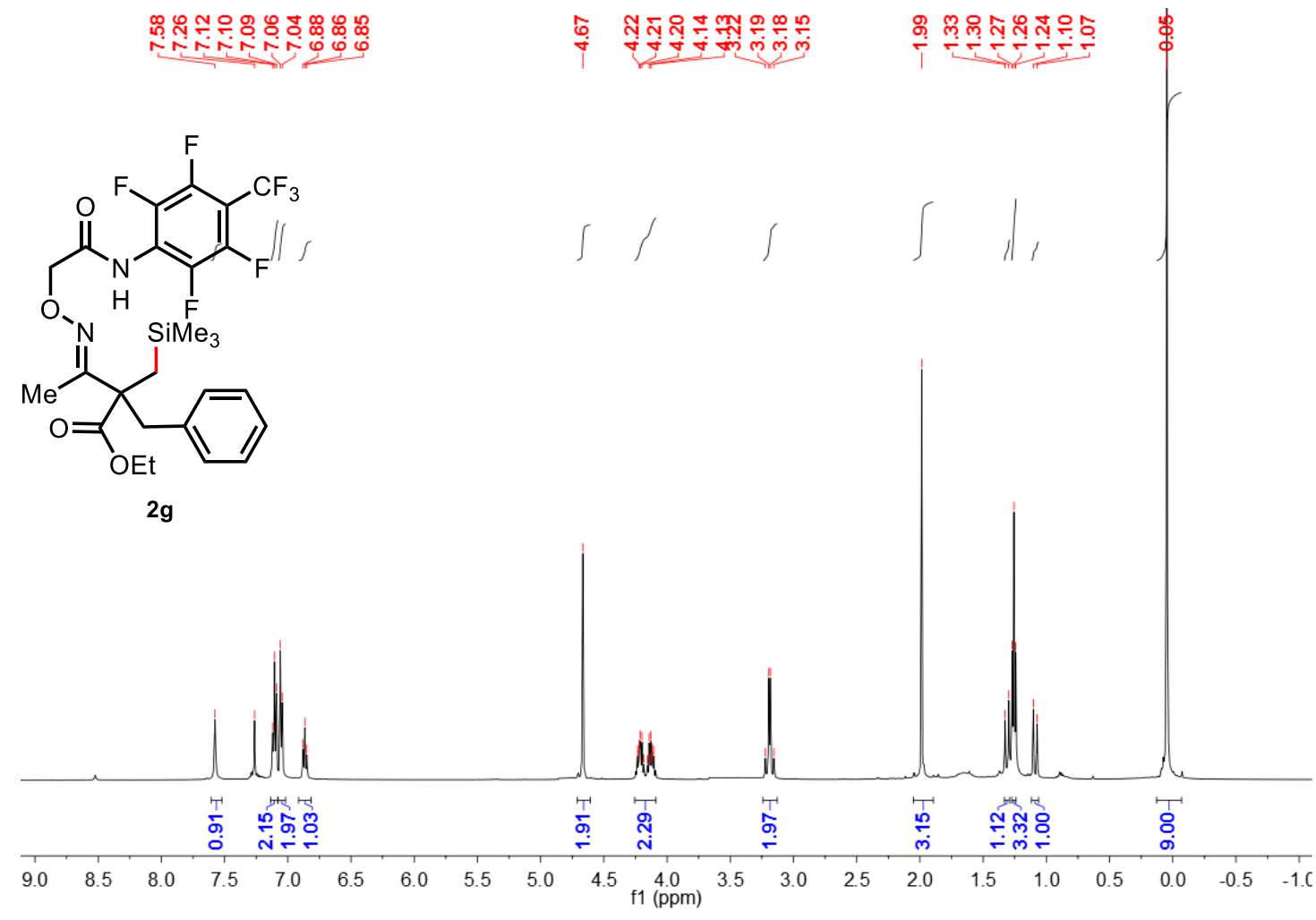

${ }^{13} \mathrm{C}$ NMR (126 MHz, $\left.\mathrm{CDCl}_{3}\right)$ spectra for $2 \mathrm{~g}$

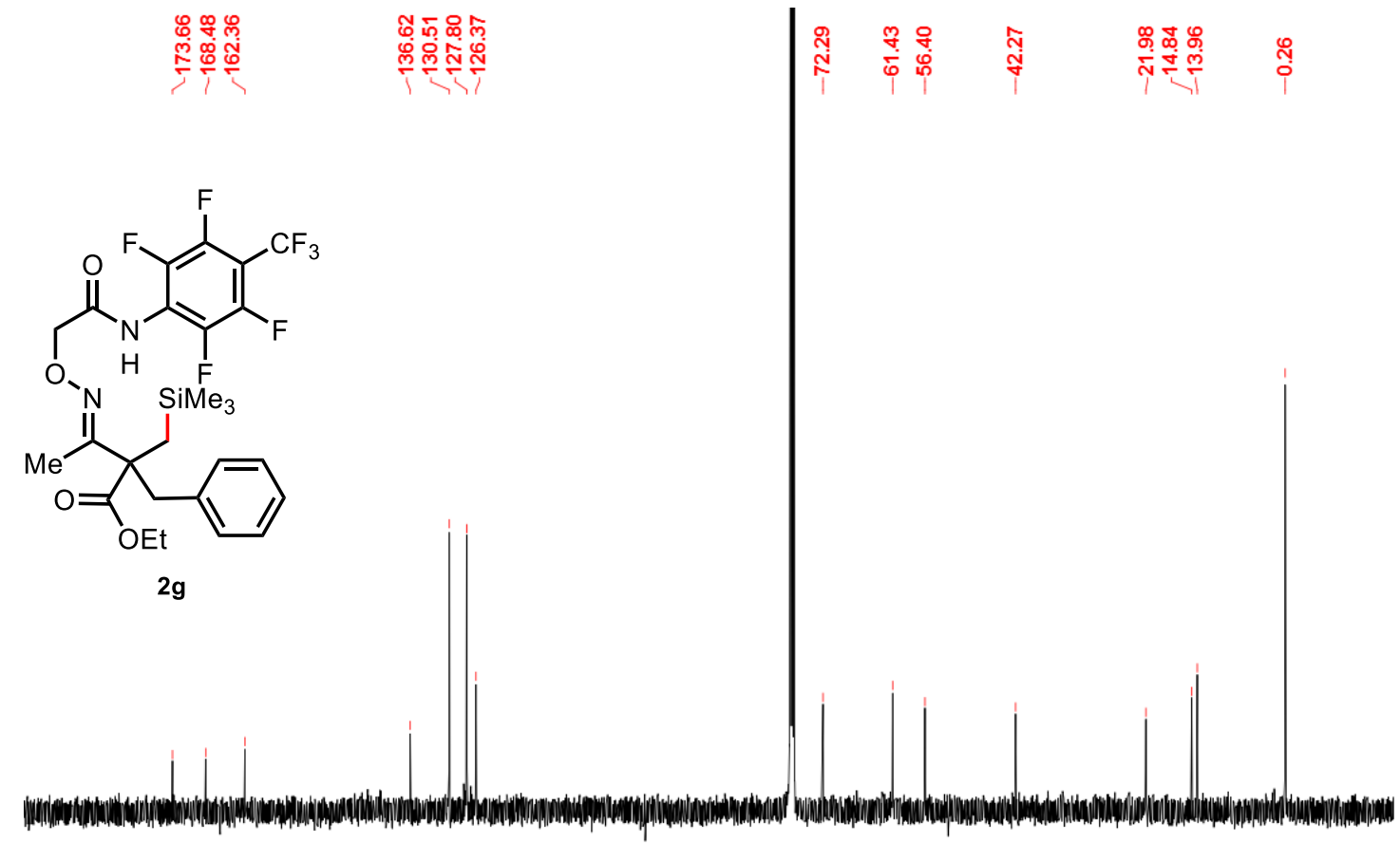

$\begin{array}{lllllllllllllllllllll}190 & 180 & 170 & 160 & 150 & 140 & 130 & 120 & 110 & 100 & \begin{array}{l}90 \\ \mathrm{f} 1(\mathrm{ppm})\end{array} & 80 & 70 & 60 & 50 & 40 & 30 & 20 & 10 & 0 & -10\end{array}$ 
${ }^{1} \mathrm{H}$ NMR (500 MHz, $\left.\mathrm{CDCl}_{3}\right)$ spectra for $2 \mathrm{~h}$

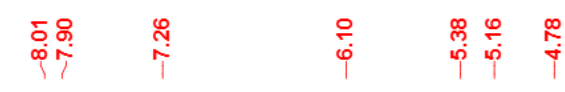

ํำ

(c)
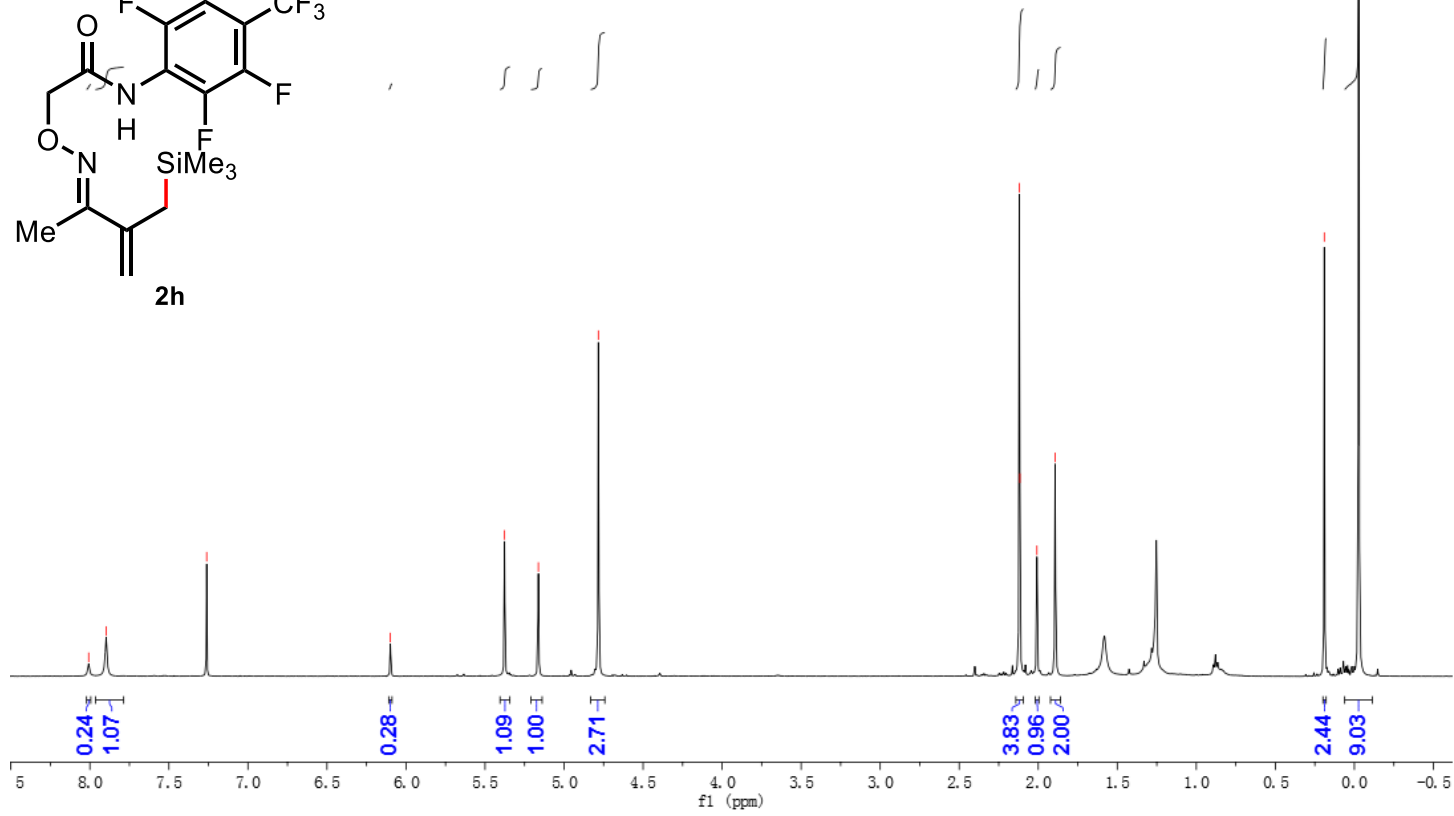

${ }^{13} \mathrm{C}$ NMR (126 MHz, $\left.\mathrm{CDCl}_{3}\right)$ spectra for $2 \mathrm{~h}$

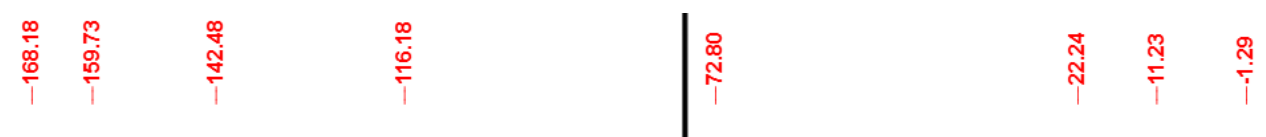<smiles></smiles><smiles>C=C(C[SiH3])C(C)=NC</smiles>

$2 \mathrm{~h}$

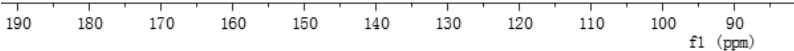


${ }^{1} \mathrm{H}$ NMR (500 MHz, $\left.\mathrm{CDCl}_{3}\right)$ spectra for $2 \mathrm{i}$

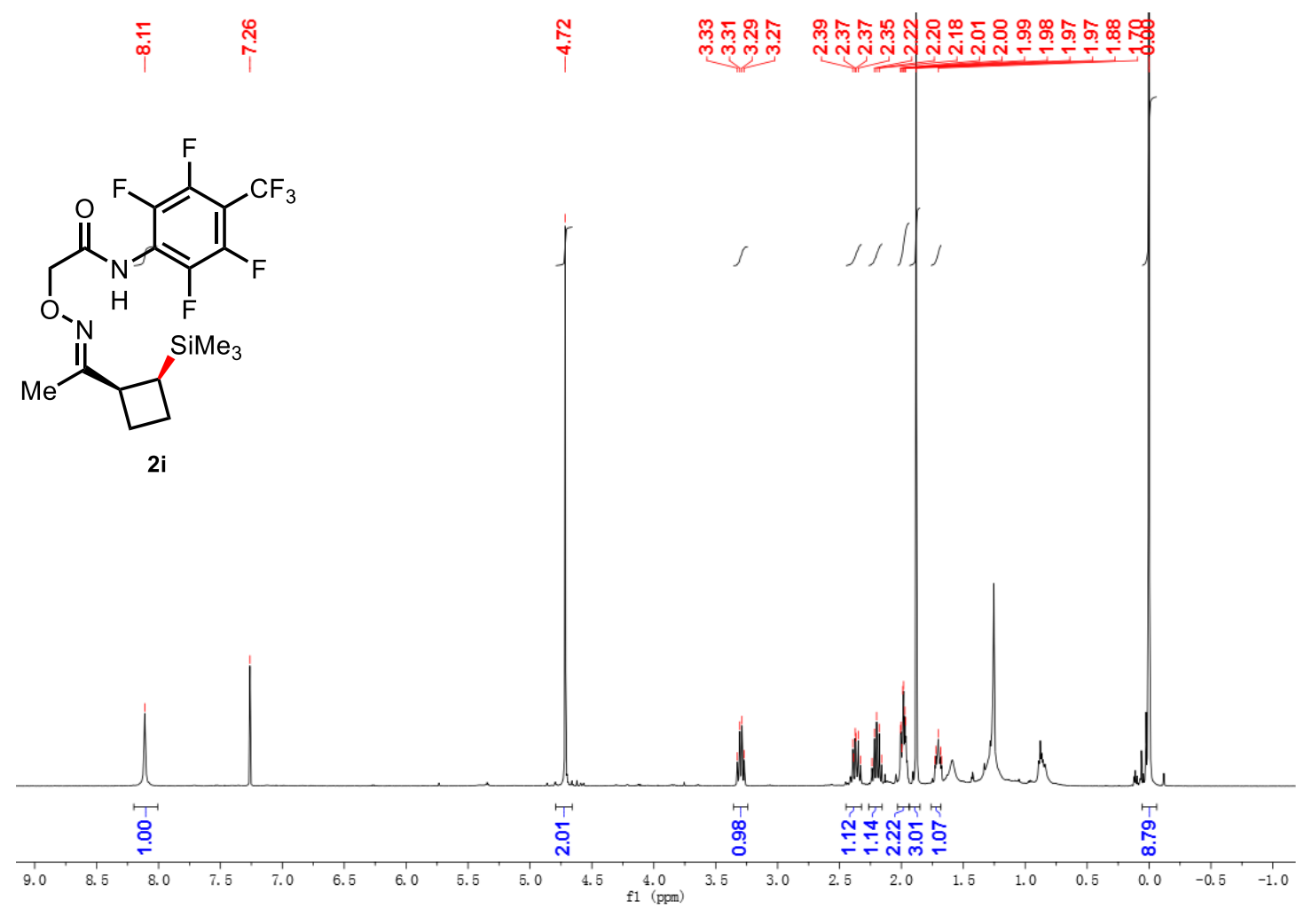

${ }^{13} \mathrm{C}$ NMR (126 MHz, $\left.\mathrm{CDCl}_{3}\right)$ spectra for $2 \mathrm{i}$

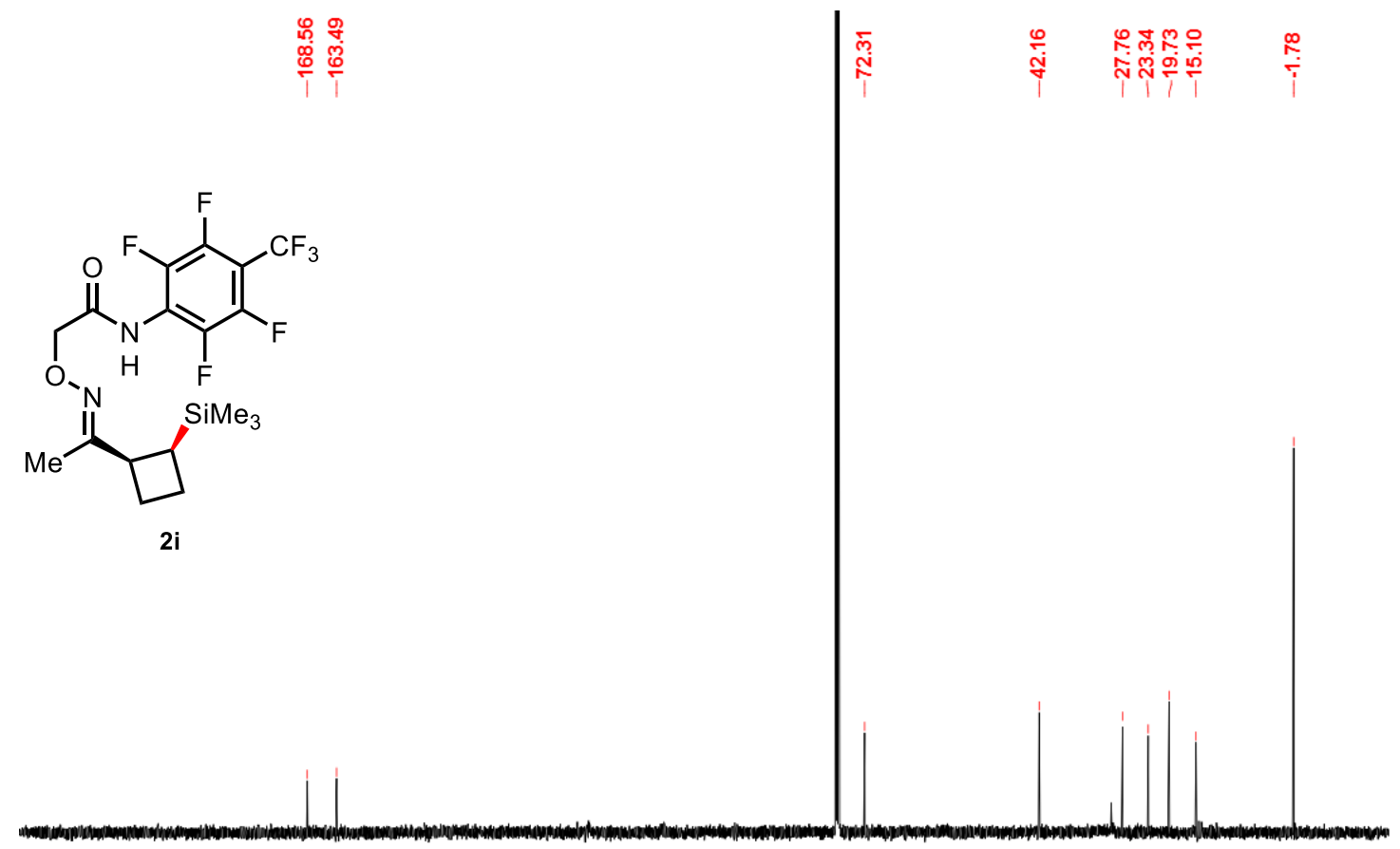

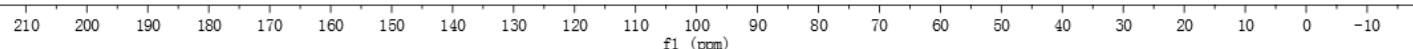


${ }^{1} \mathrm{H}$ NMR (500 MHz, $\left.\mathrm{CDCl}_{3}\right)$ spectra for $2 \mathrm{j}$

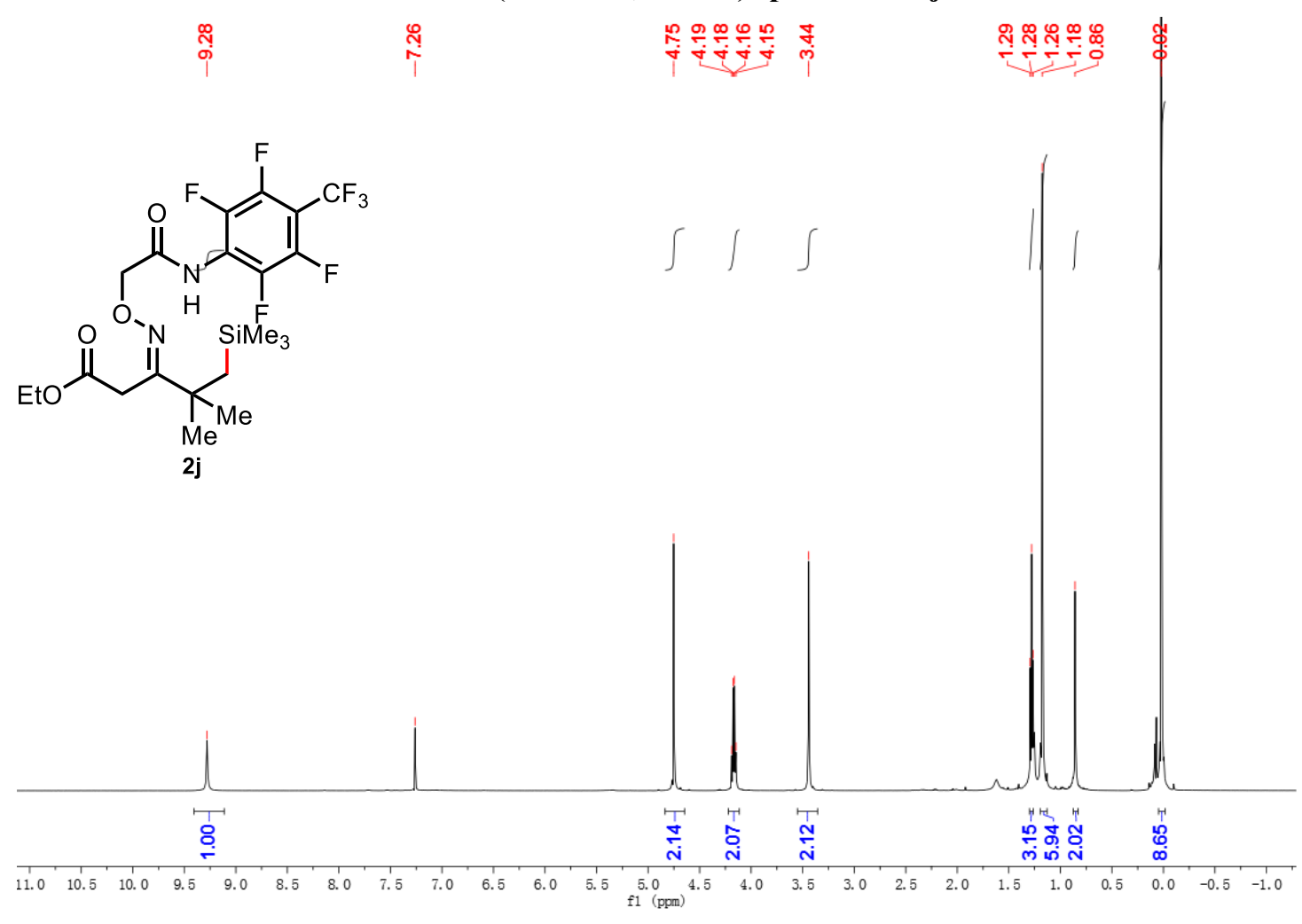

${ }^{13} \mathrm{C}$ NMR (126 MHz, $\mathrm{CDCl}_{3}$ ) spectra for $2 \mathrm{j}$

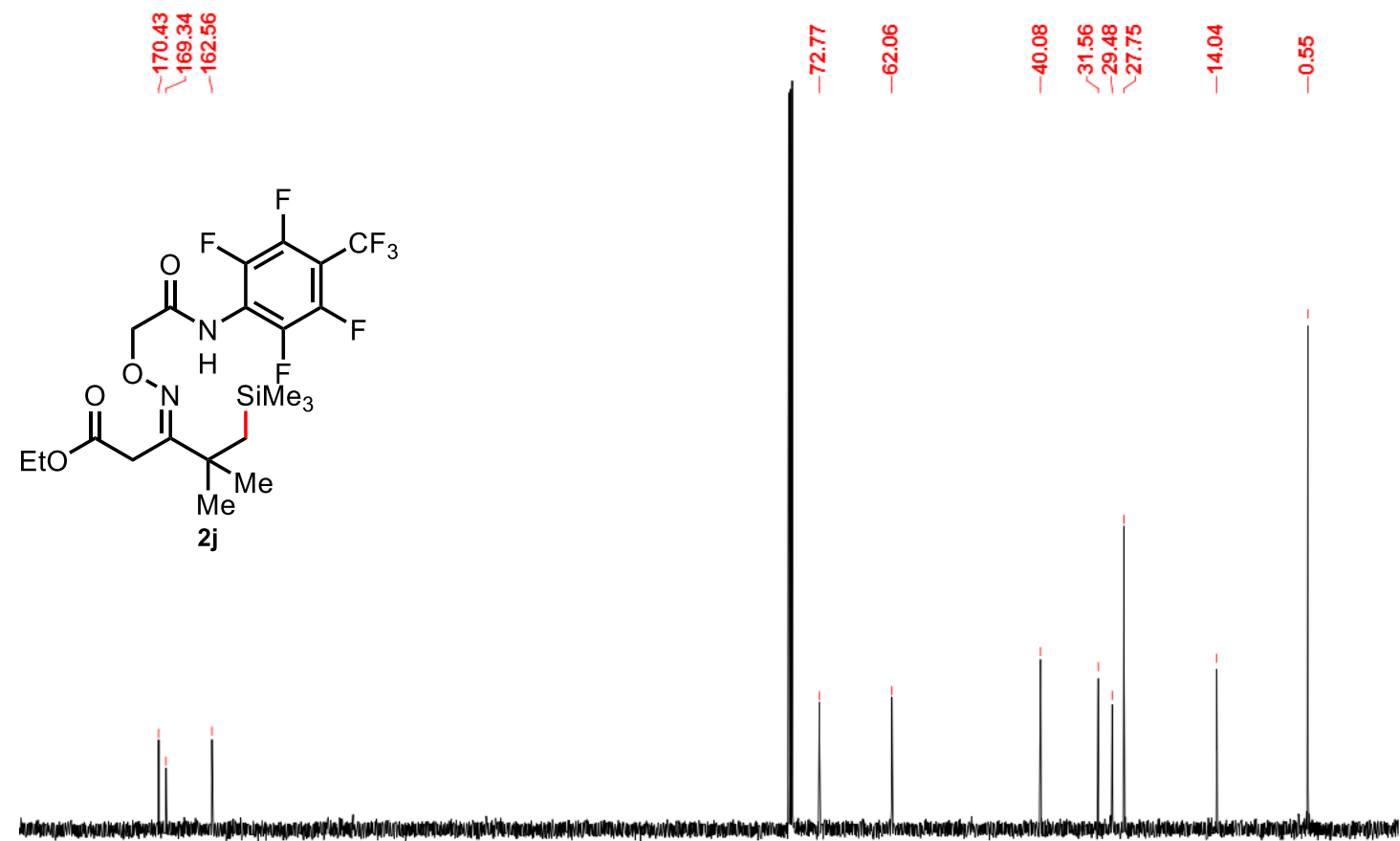

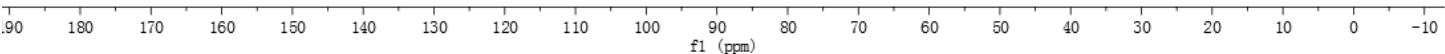


${ }^{1} \mathrm{H}$ NMR (500 MHz, $\mathrm{CDCl}_{3}$ ) spectra for $2 \mathrm{k}$

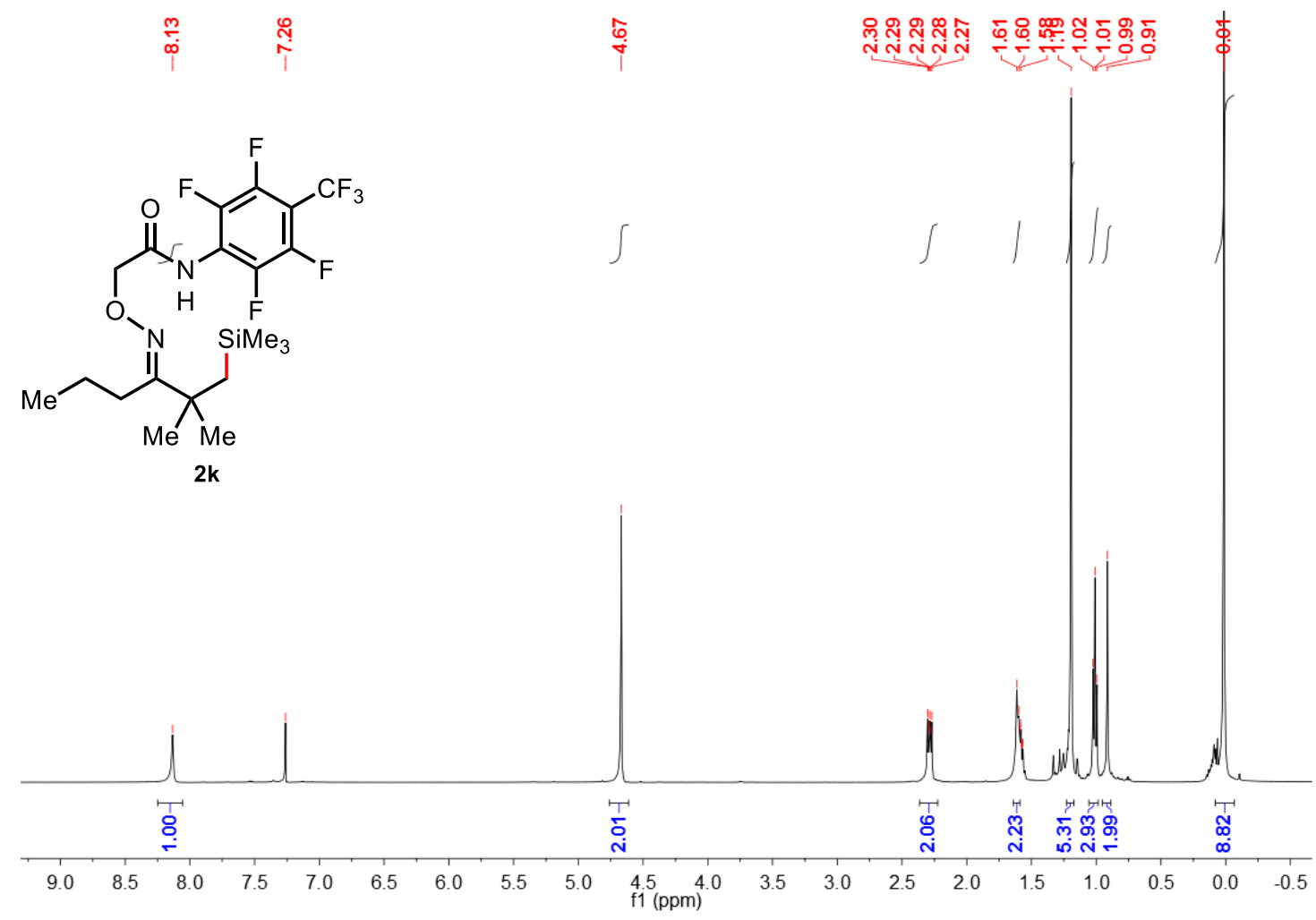

${ }^{13} \mathrm{C}$ NMR (126 MHz, $\mathrm{CDCl}_{3}$ ) spectra for $2 \mathrm{k}$
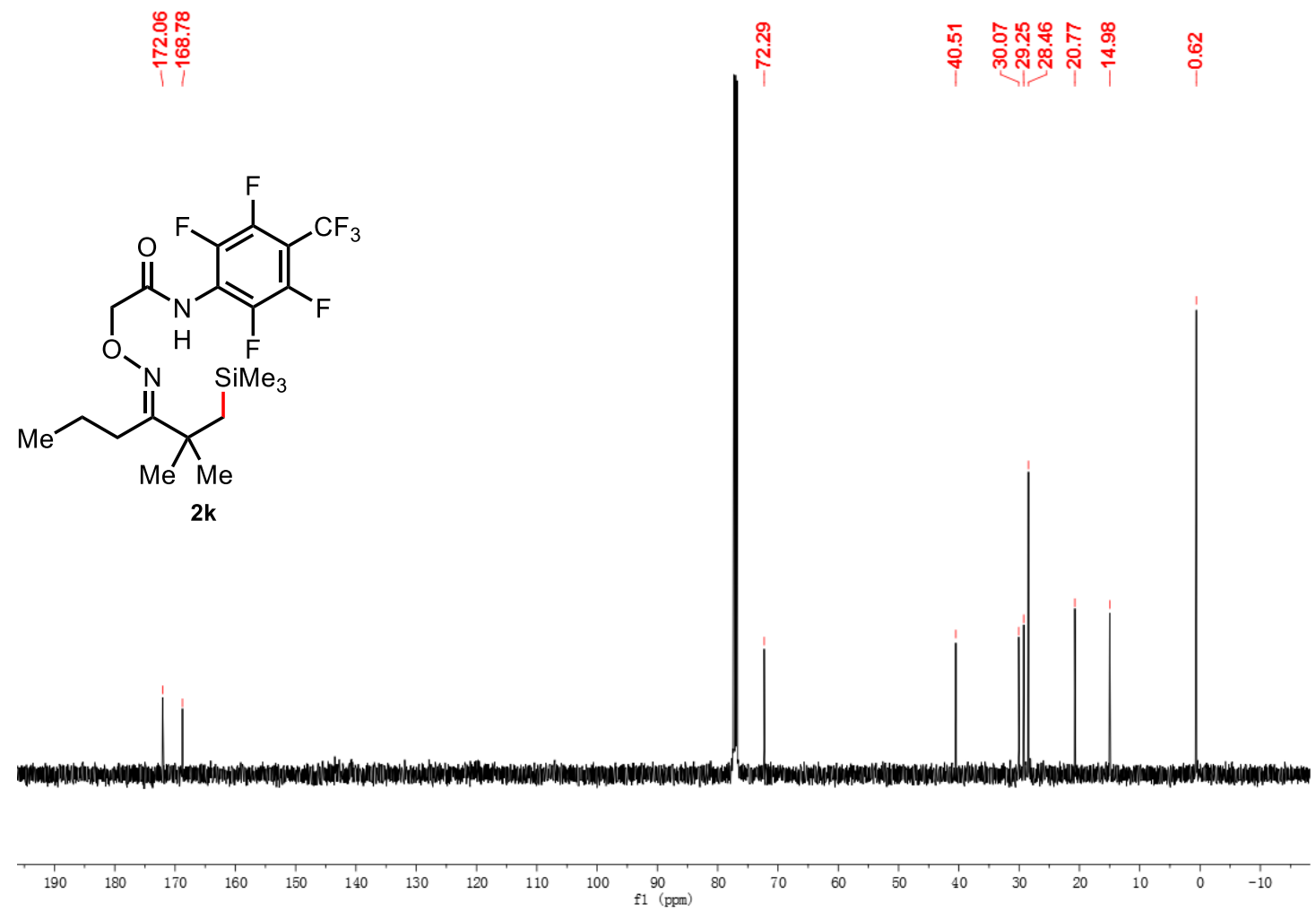


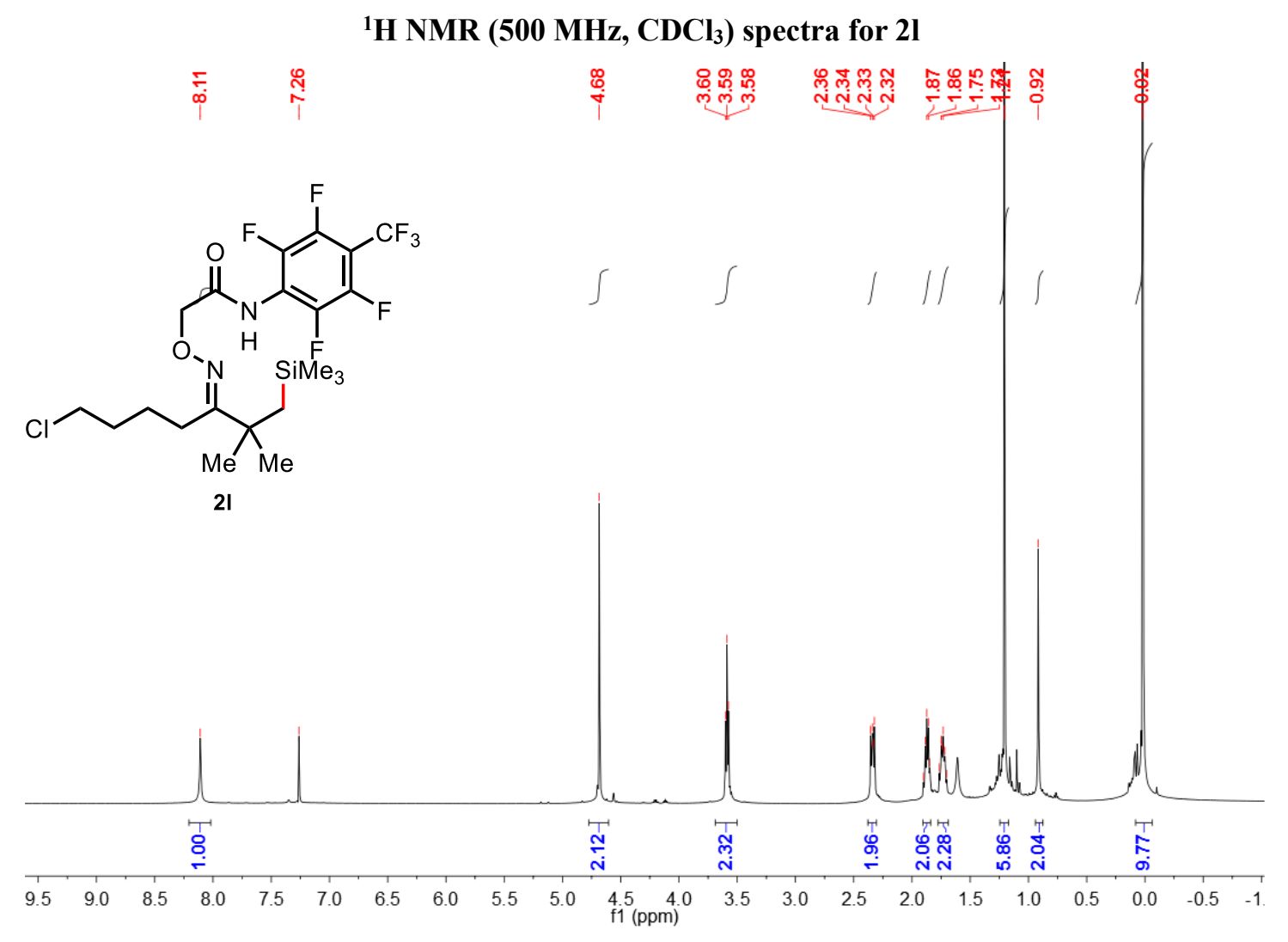

${ }^{1}$ H NMR (500 MHz, CDCl3) spectra for $2 I$

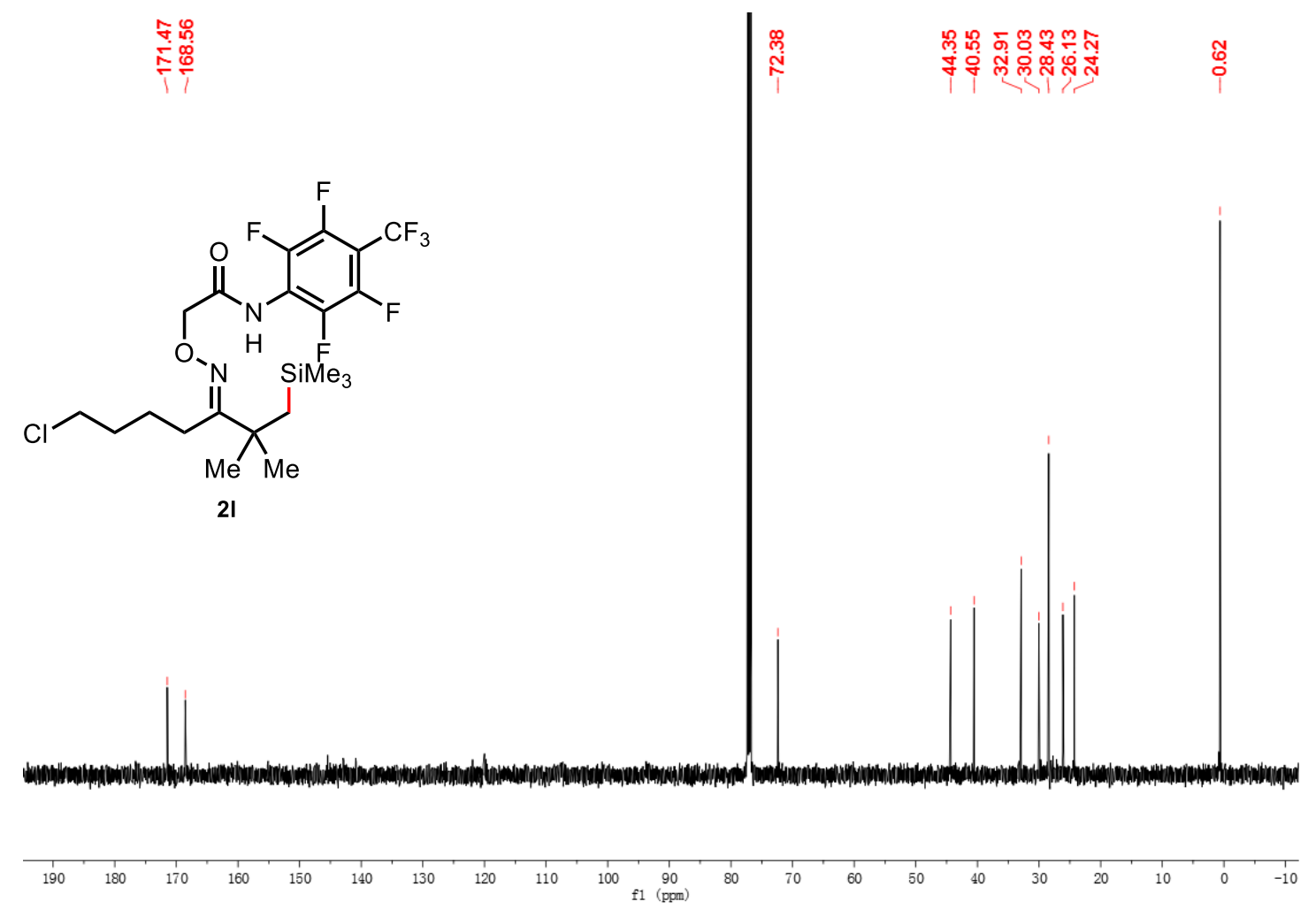


${ }^{1} \mathrm{H}$ NMR (500 MHz, $\left.\mathrm{CDCl}_{3}\right)$ spectra for $2 \mathrm{~m}$

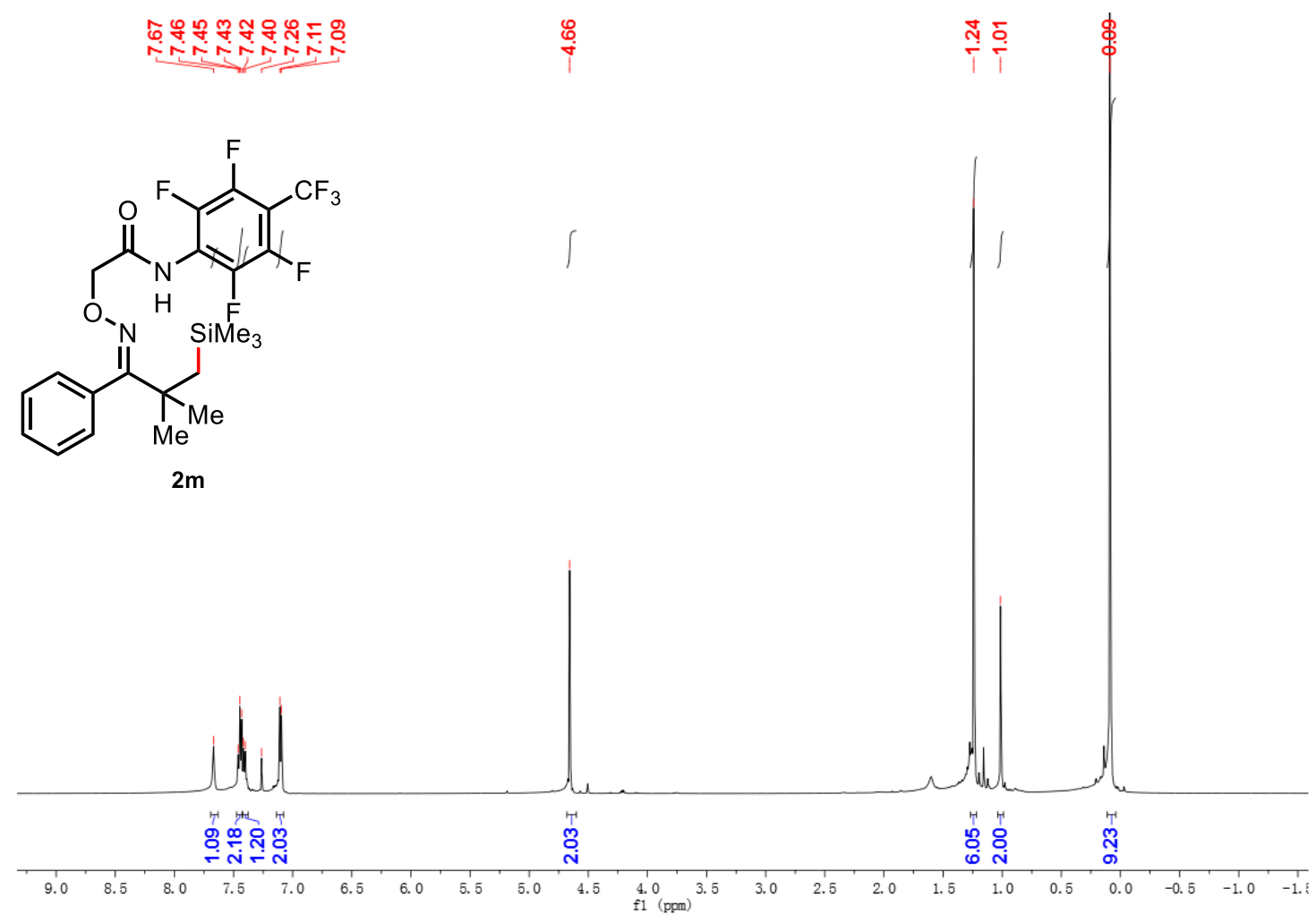

${ }^{13} \mathrm{C}$ NMR (126 MHz, $\mathrm{CDCl}_{3}$ ) spectra for $2 \mathrm{~m}$

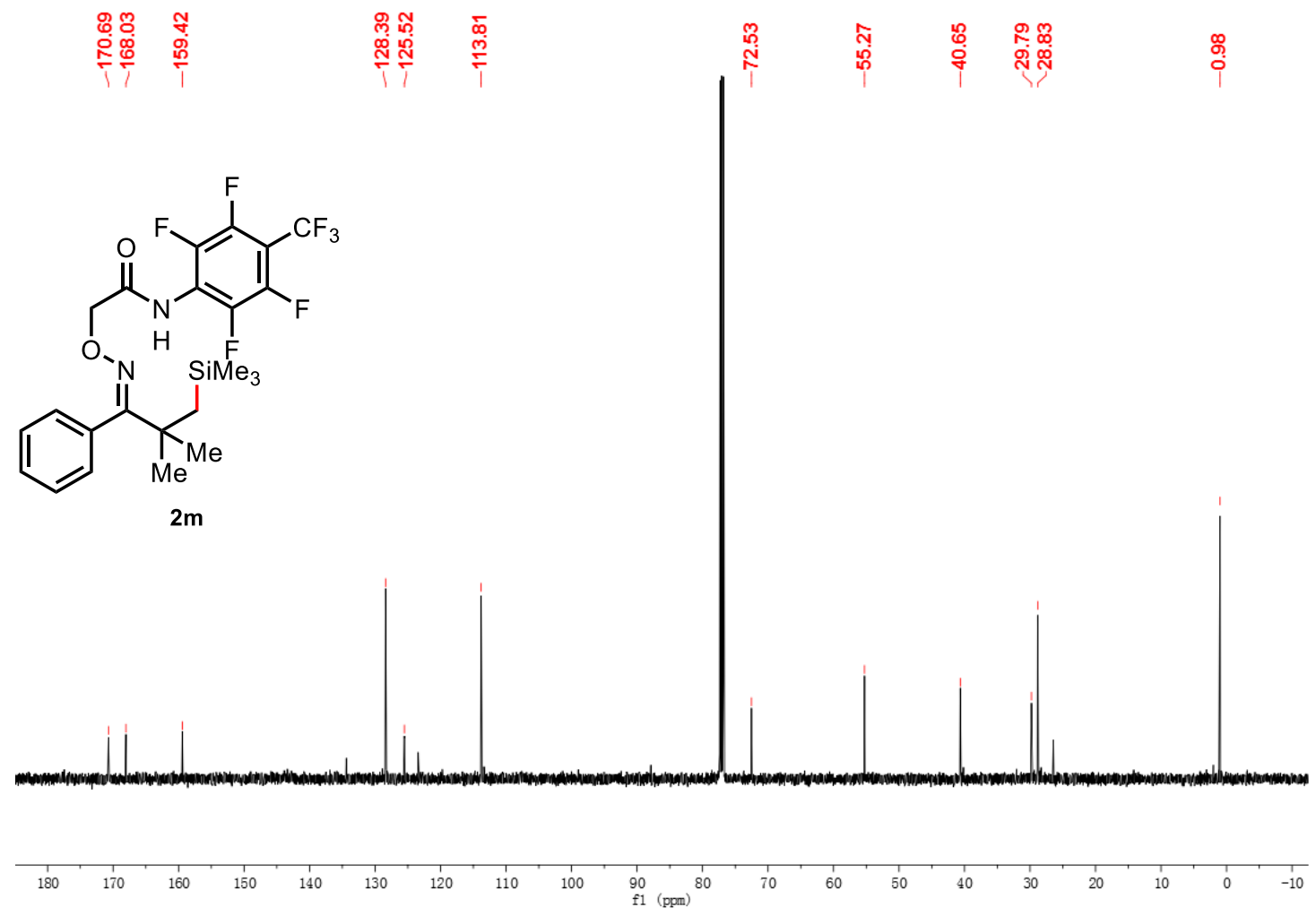


${ }^{1} \mathrm{H}$ NMR (500 MHz, $\mathrm{CDCl}_{3}$ ) spectra for $2 \mathrm{n}$

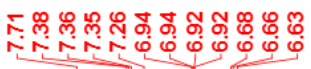

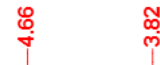<smiles>NOCC(=O)Nc1c(F)c(F)c(C(F)(F)F)c(F)c1F</smiles><smiles>COCCOCCOCCC(C)(C)C(=N)c1cccc(OC)c1</smiles>

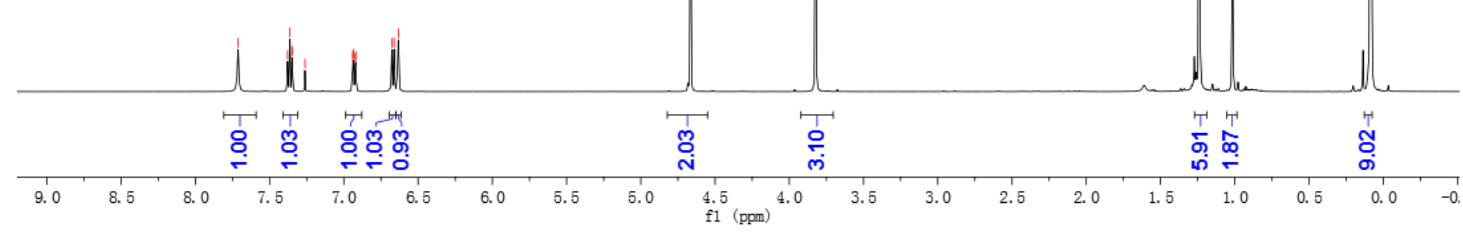

${ }^{13} \mathrm{C}$ NMR (126 MHz, $\mathrm{CDCl}_{3}$ ) spectra for $2 \mathrm{n}$
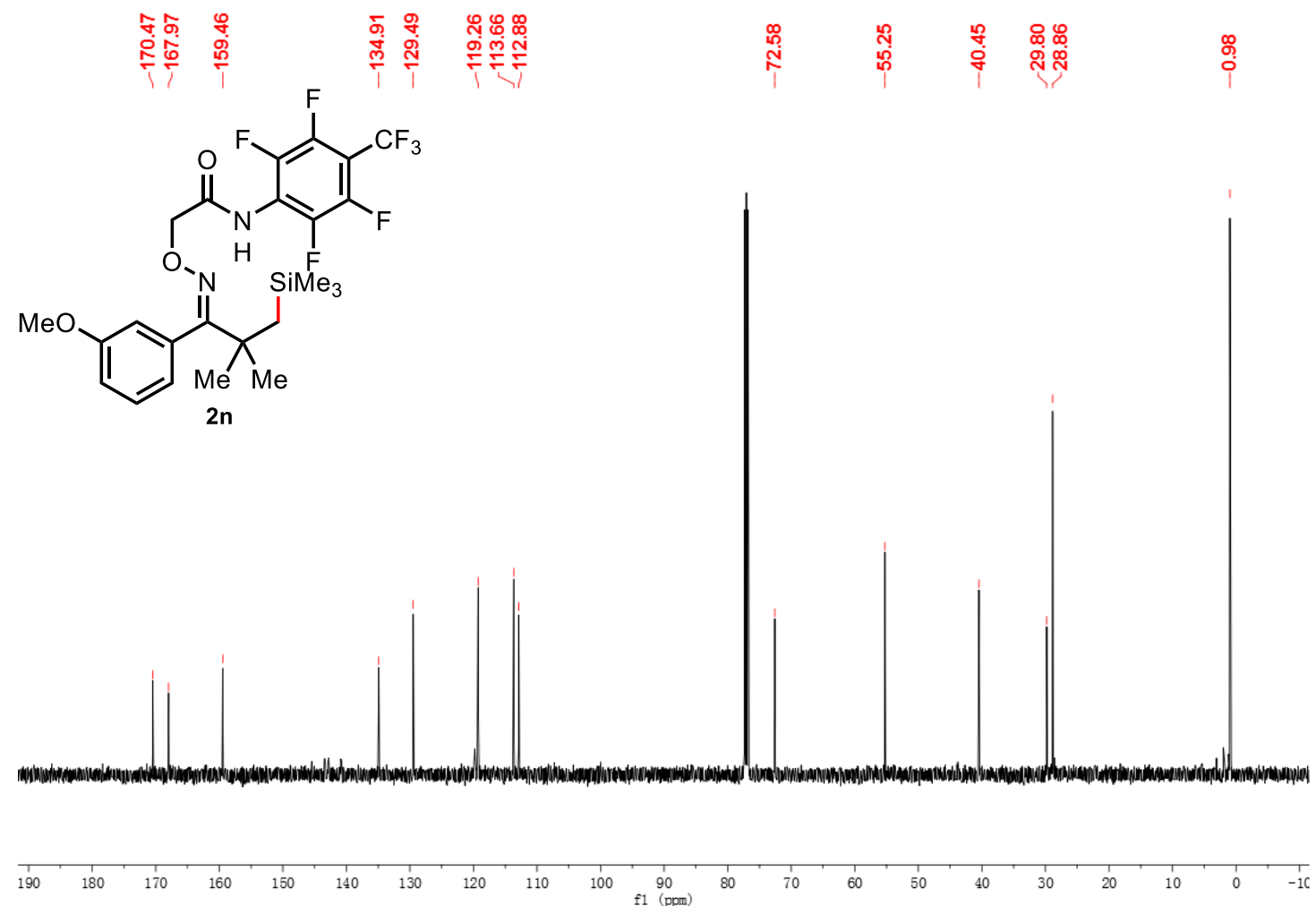
${ }^{1} \mathrm{H}$ NMR (500 MHz, $\left.\mathrm{CDCl}_{3}\right)$ spectra for 20

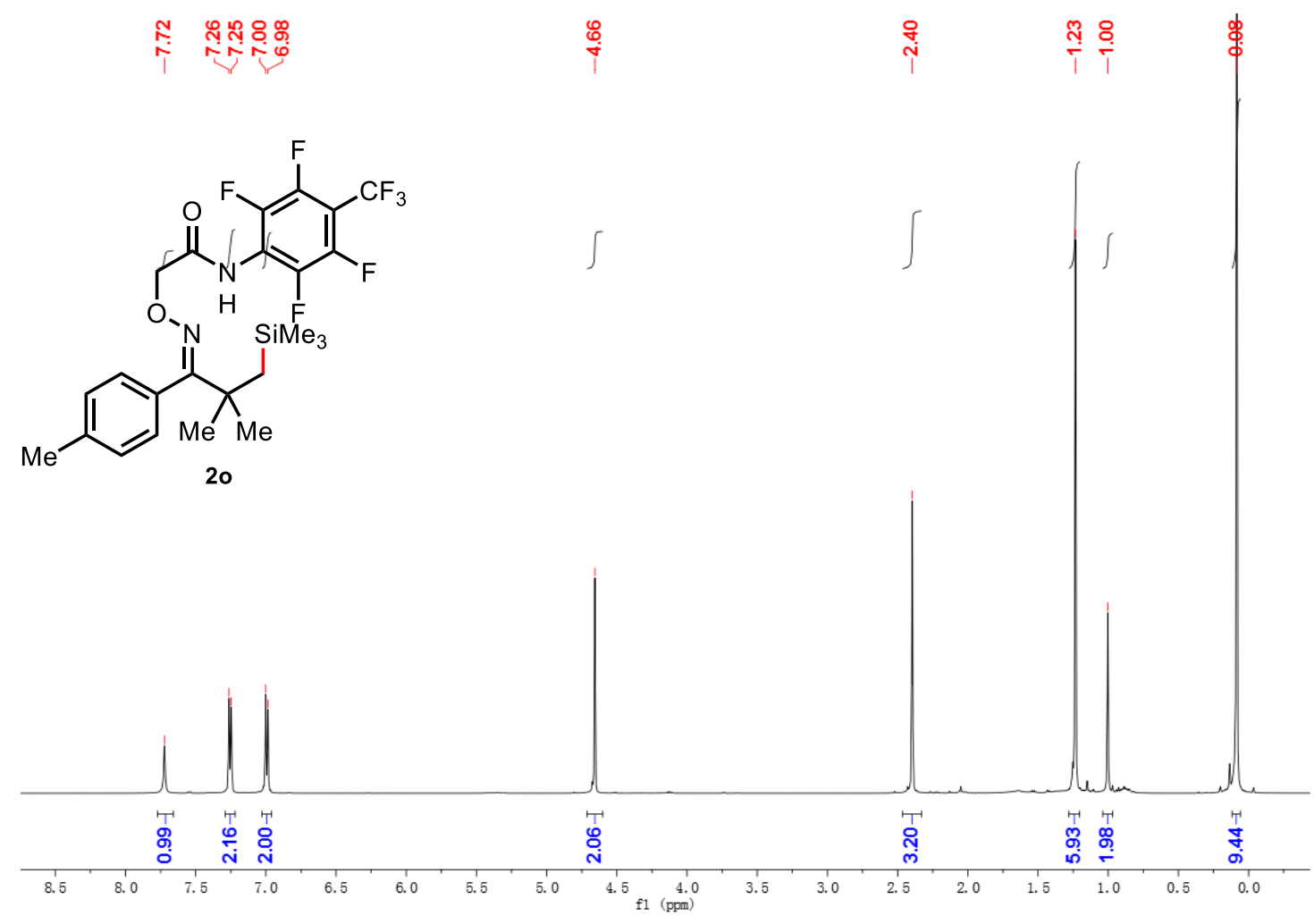

${ }^{13} \mathrm{C}$ NMR (126 MHz, $\left.\mathrm{CDCl}_{3}\right)$ spectra for 20

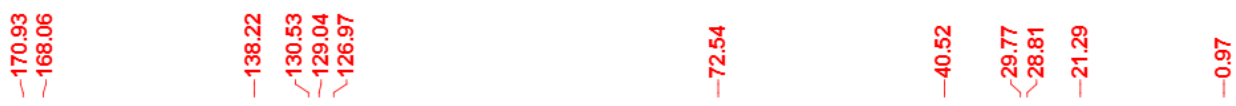

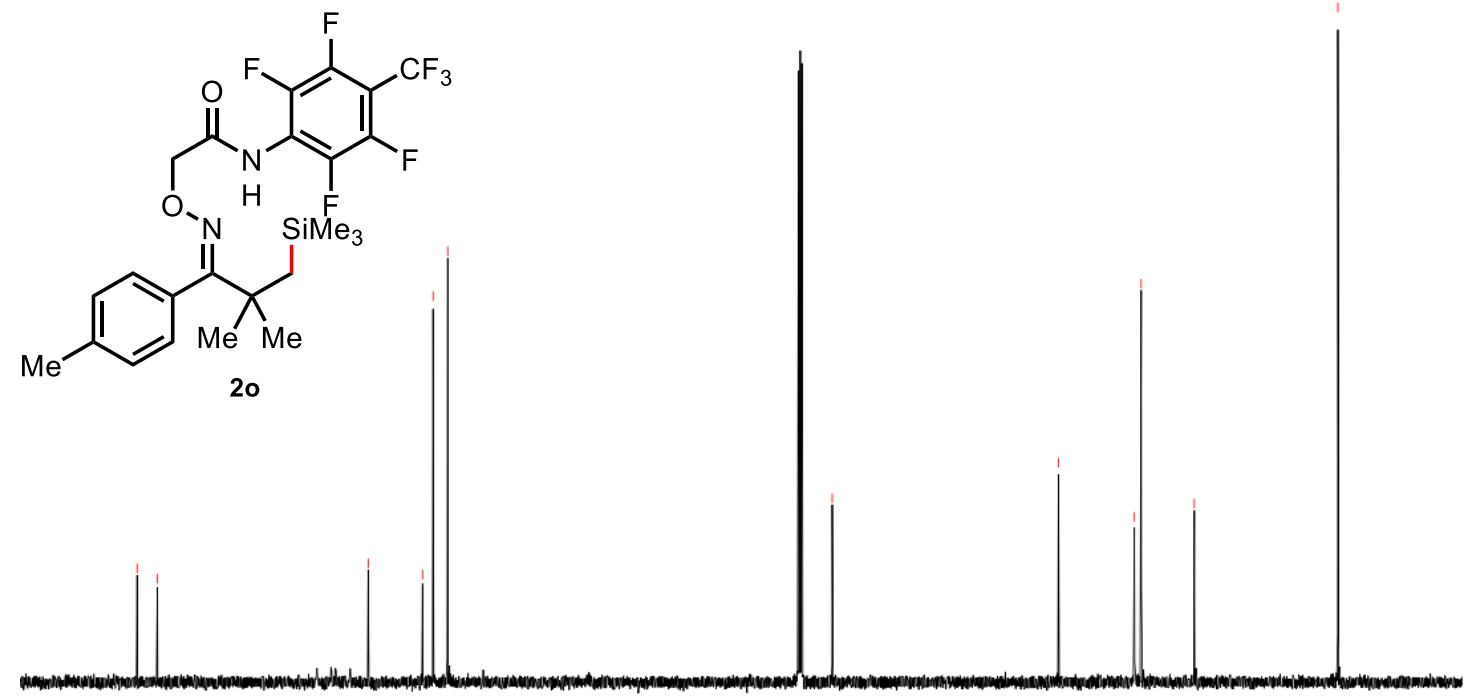

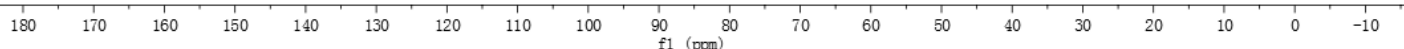


${ }^{1} \mathrm{H}$ NMR (500 MHz, $\left.\mathrm{CDCl}_{3}\right)$ spectra for $2 \mathrm{p}$

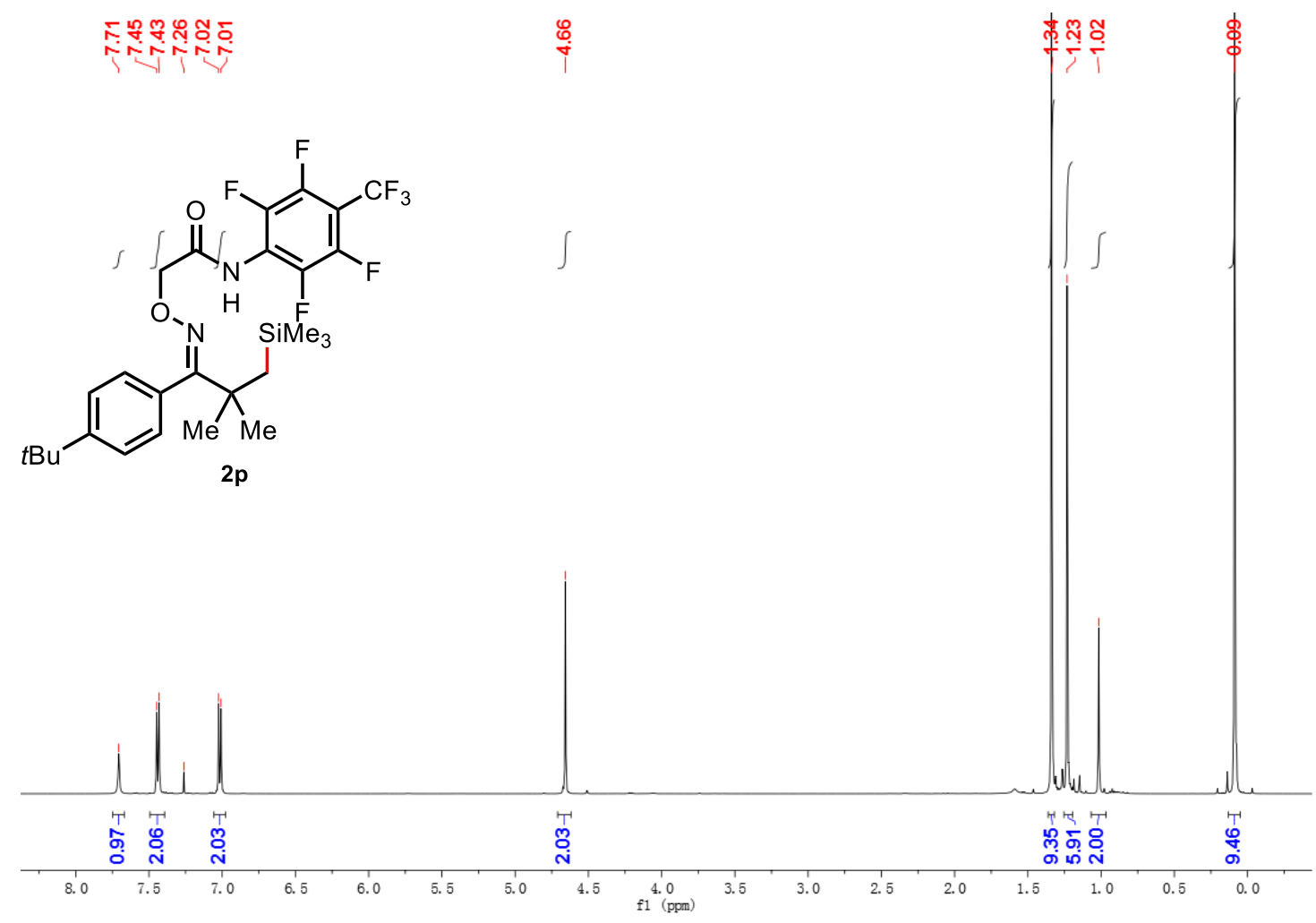

${ }^{13} \mathrm{C}$ NMR (126 MHz, $\mathrm{CDCl}_{3}$ ) spectra for 2p

\begin{tabular}{|c|c|c|c|}
\hline$\stackrel{\bar{s}}{\stackrel{5}{i}}$ & 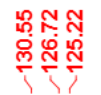 & $\stackrel{\text { N }}{\text { i }}$ & 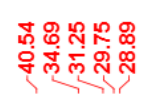 \\
\hline
\end{tabular}<smiles>CCC(C)(C)/C(=N\OCC(=O)Nc1c(F)c(F)c(C(F)(F)F)c(F)c1F)c1ccc(C(C)(C)C)cc1</smiles>

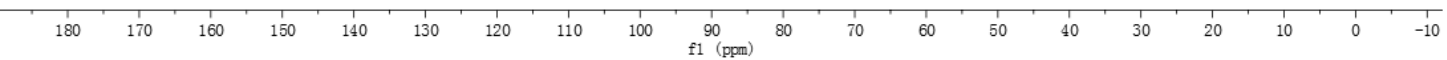


${ }^{1} \mathrm{H}$ NMR (500 MHz, $\left.\mathrm{CDCl}_{3}\right)$ spectra for $2 \mathrm{q}$

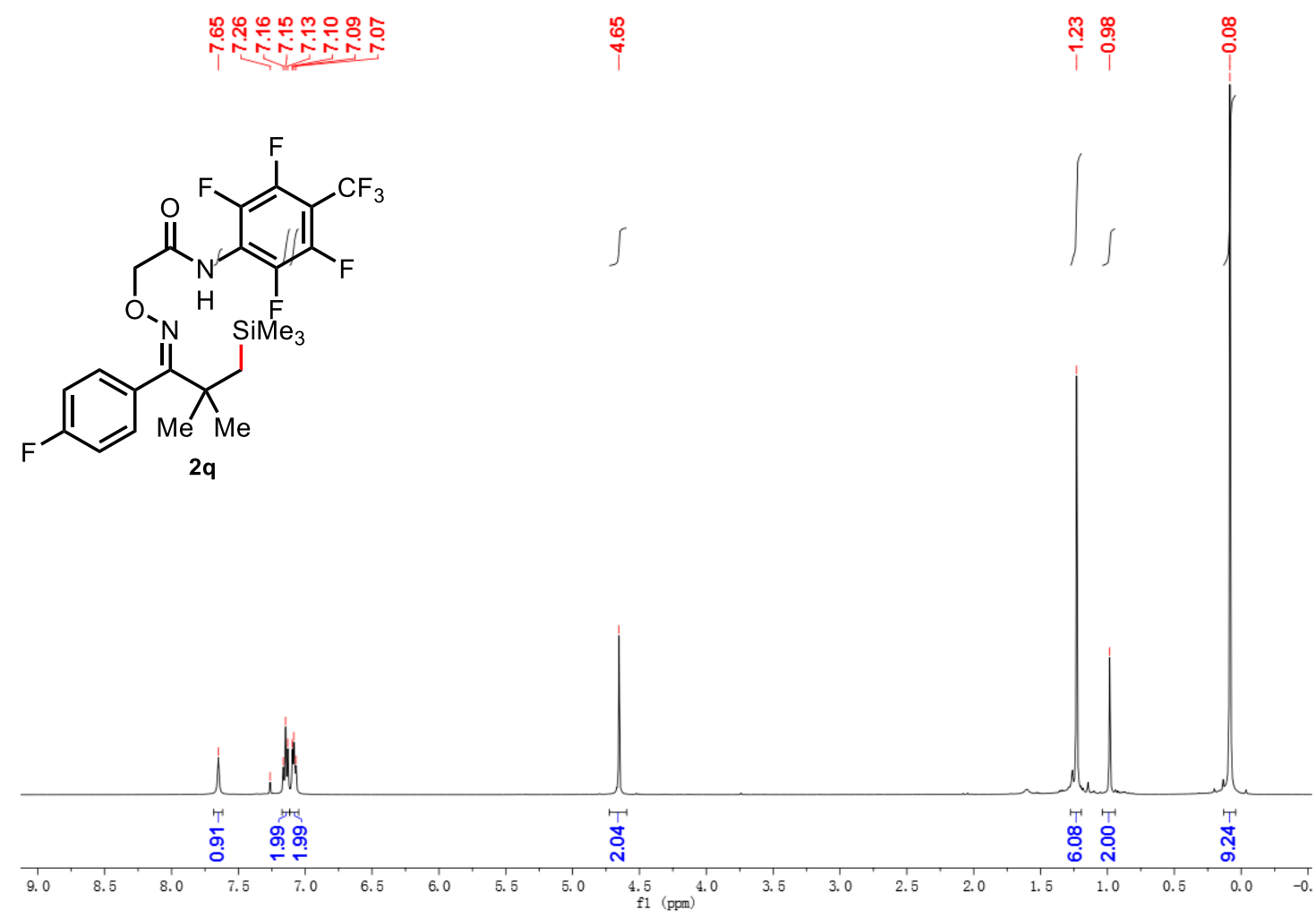

${ }^{13} \mathrm{C}$ NMR (126 MHz, $\mathrm{CDCl}_{3}$ ) spectra for $2 \mathrm{q}$

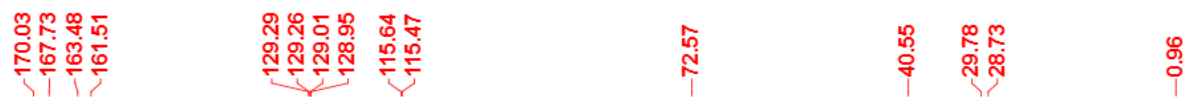<smiles>CCC(C)(C)C(=NOCC(=O)Nc1c(F)c(F)c(C(F)(F)F)c(F)c1F)c1ccc(F)cc1</smiles>

2q

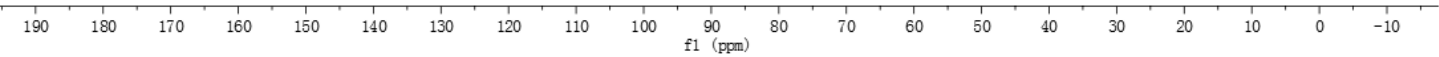


${ }^{1} \mathrm{H}$ NMR (500 MHz, $\mathrm{CDCl}_{3}$ ) spectra for $2 \mathrm{r}$

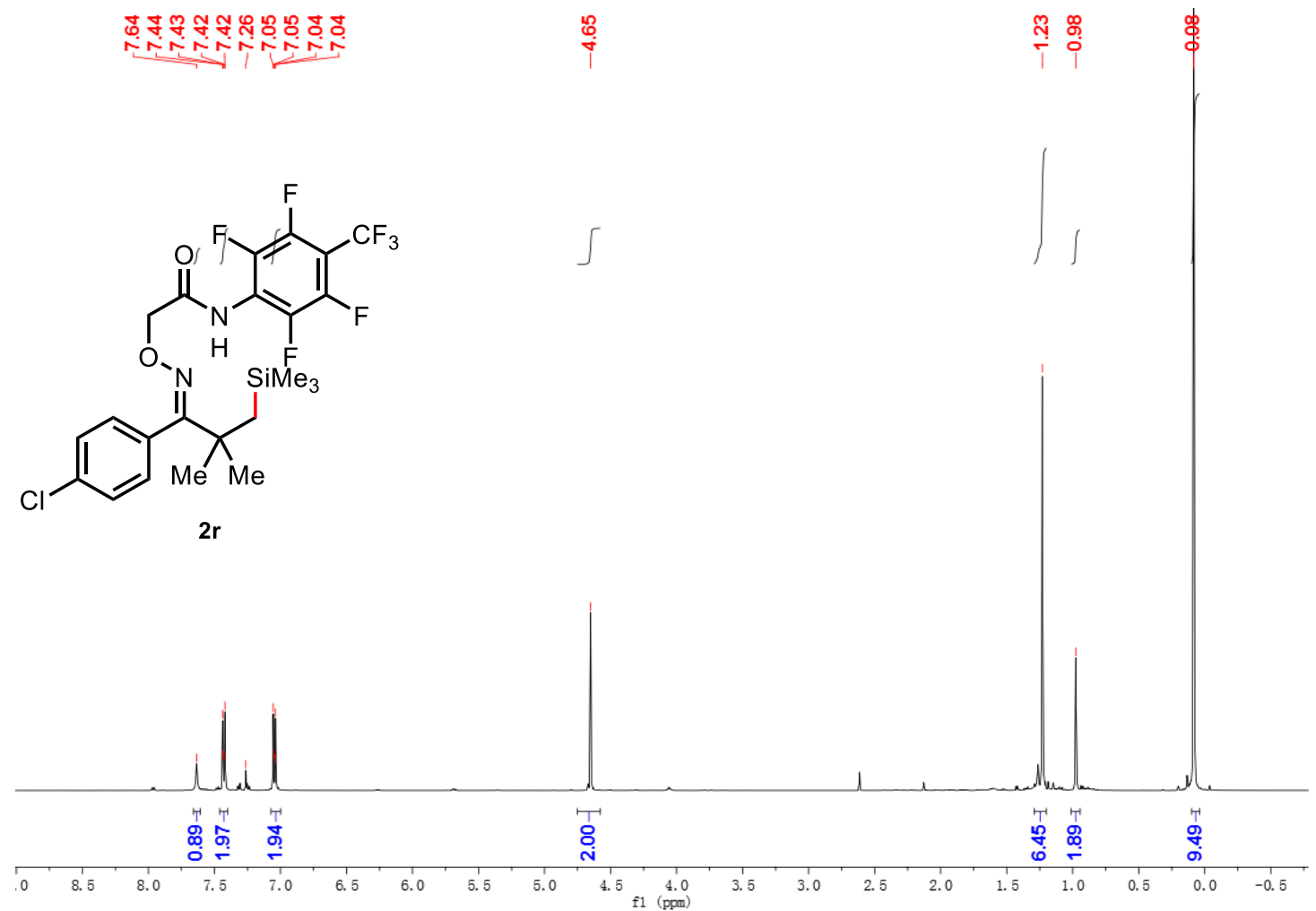

${ }^{13} \mathrm{C}$ NMR (126 MHz, $\mathrm{CDCl}_{3}$ ) spectra for $2 \mathrm{r}$

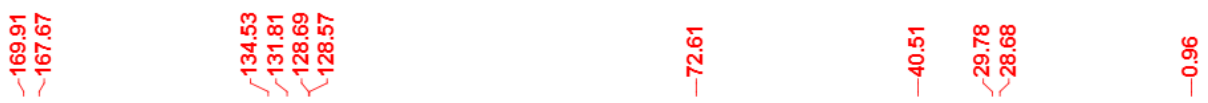

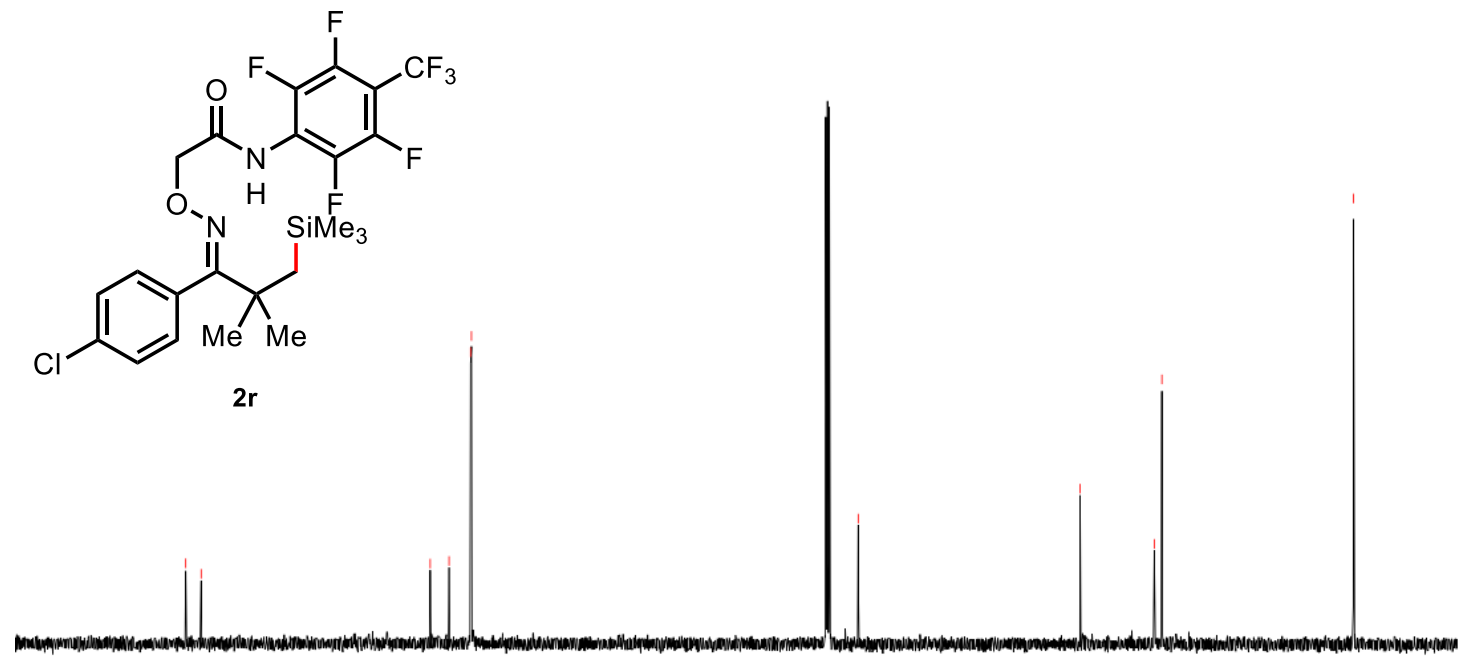

$\begin{array}{lllllllllllllllllllllll}1 & 1 & 180 & 170 & 160 & 150 & 140 & 130 & 120 & 110 & 100 & 90 & 80 & 70 & 60 & 50 & 40 & 30 & 20 & 1 & 0 & -10\end{array}$ 
${ }^{1} \mathrm{H}$ NMR (500 MHz, $\mathrm{CDCl}_{3}$ ) spectra for $2 \mathrm{~s}$

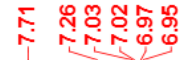<smiles>C[18O]c1c(F)c(C(F)(F)F)c(F)c(F)c1NC(=O)CON</smiles><smiles>CCCCC(C)(C)C(=N)c1ccc(OC)cc1</smiles>
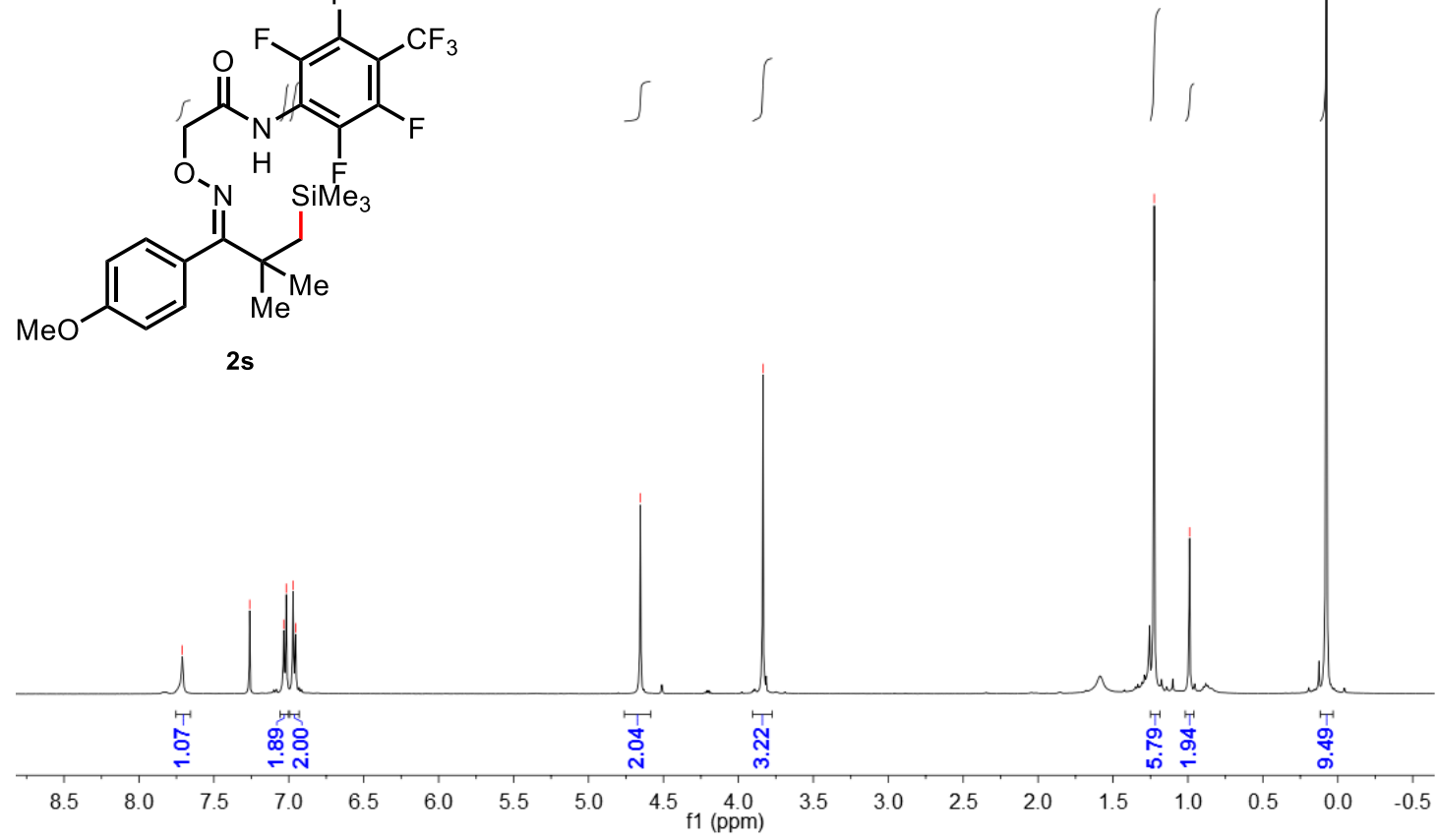

${ }^{13} \mathrm{C}$ NMR (126 MHz, $\mathrm{CDCl}_{3}$ ) spectra for $2 \mathrm{~s}$

$$
\text { 융 ๆ }
$$

윰<smiles>CC(C)c1c(F)c(C(F)(F)F)c(F)c(F)c1NC(=O)CON</smiles><smiles>CO/N=C(/c1ccc(OC)cc1)C(C)(C)C[13CH3]</smiles>

2s

$\begin{array}{llllllllll}180 & 170 & 160 & 150 & 140 & 130 & 120 & 110 & 100 & \begin{array}{c}90 \\ \mathrm{f} 1(\mathrm{ppm})\end{array} 80\end{array}$

$\begin{array}{lllllllll}70 & 60 & 50 & 40 & 30 & 20 & 10 & 0 & -10\end{array}$ 
${ }^{1} \mathrm{H}$ NMR (500 MHz, $\mathrm{CDCl}_{3}$ ) spectra for $2 \mathrm{t}$

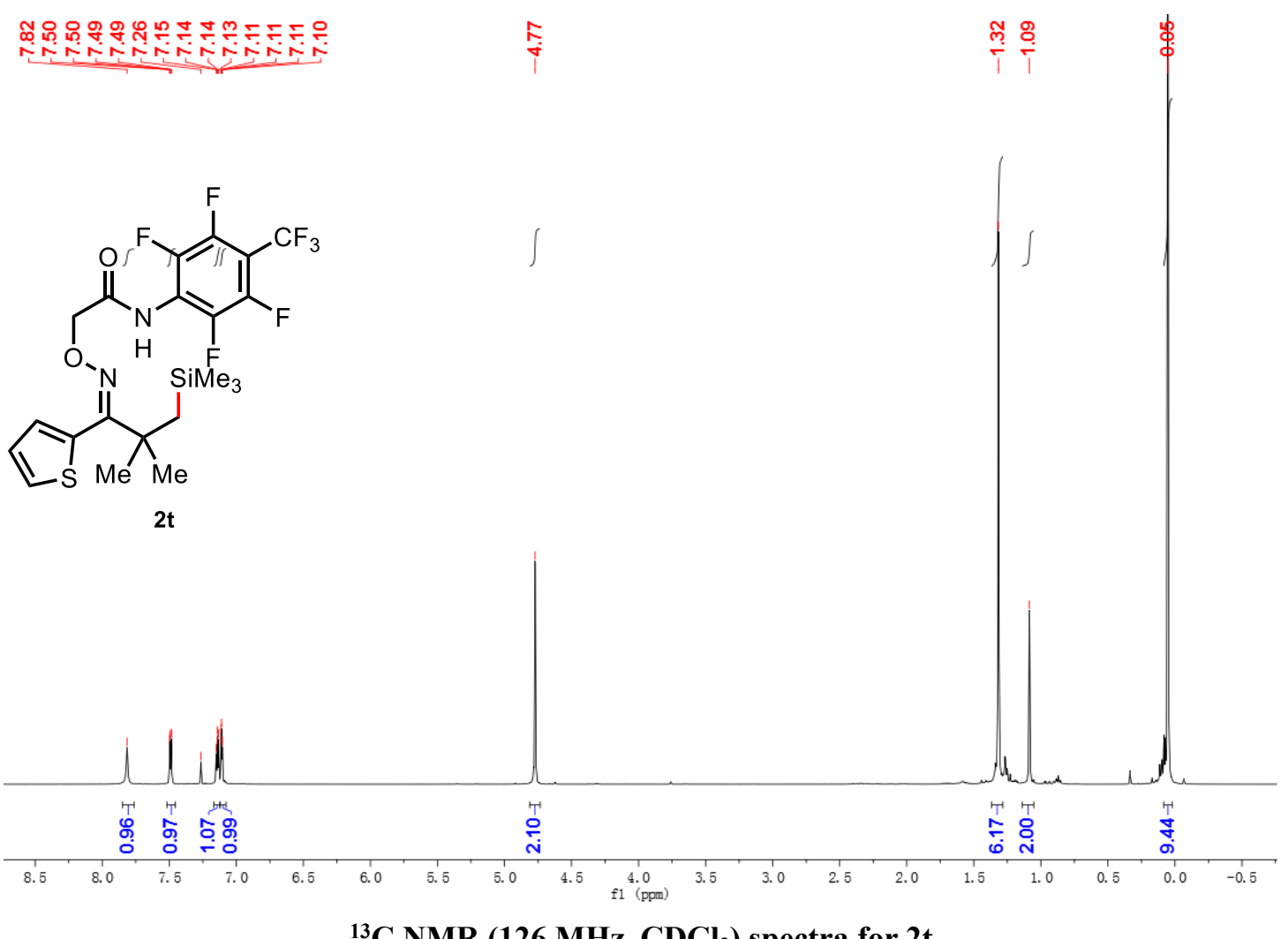

${ }^{13} \mathrm{C}$ NMR (126 $\left.\mathrm{MHz}, \mathrm{CDCl}_{3}\right)$ spectra for $2 \mathrm{t}$
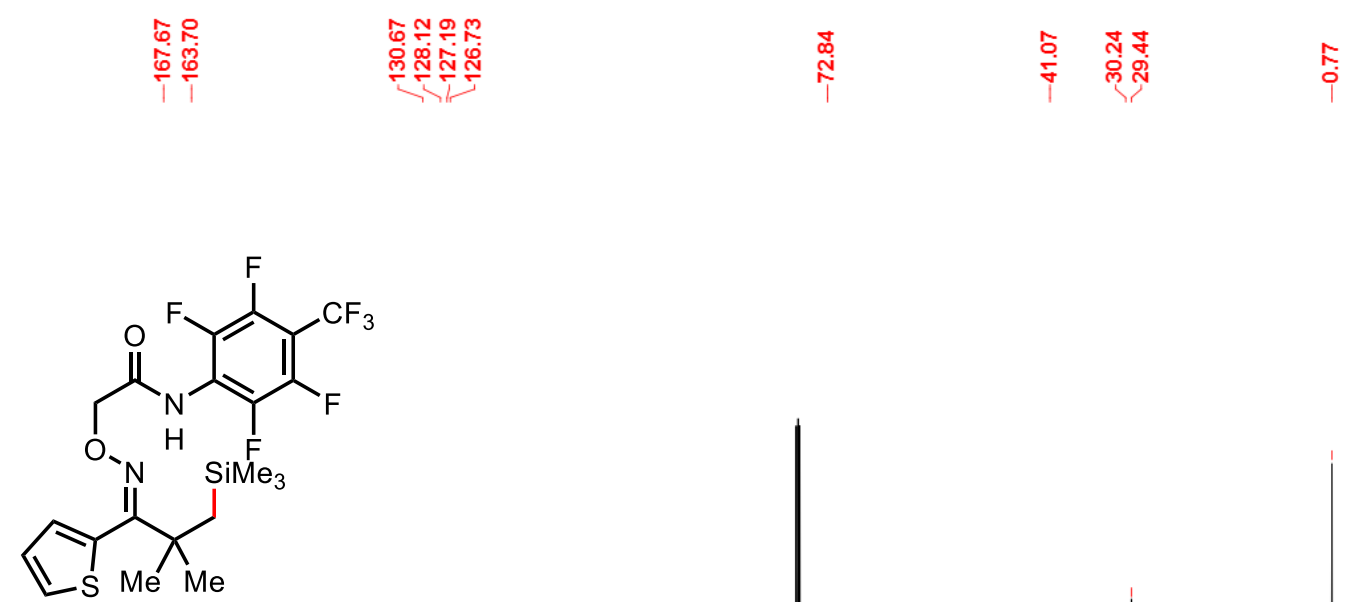

$2 t$
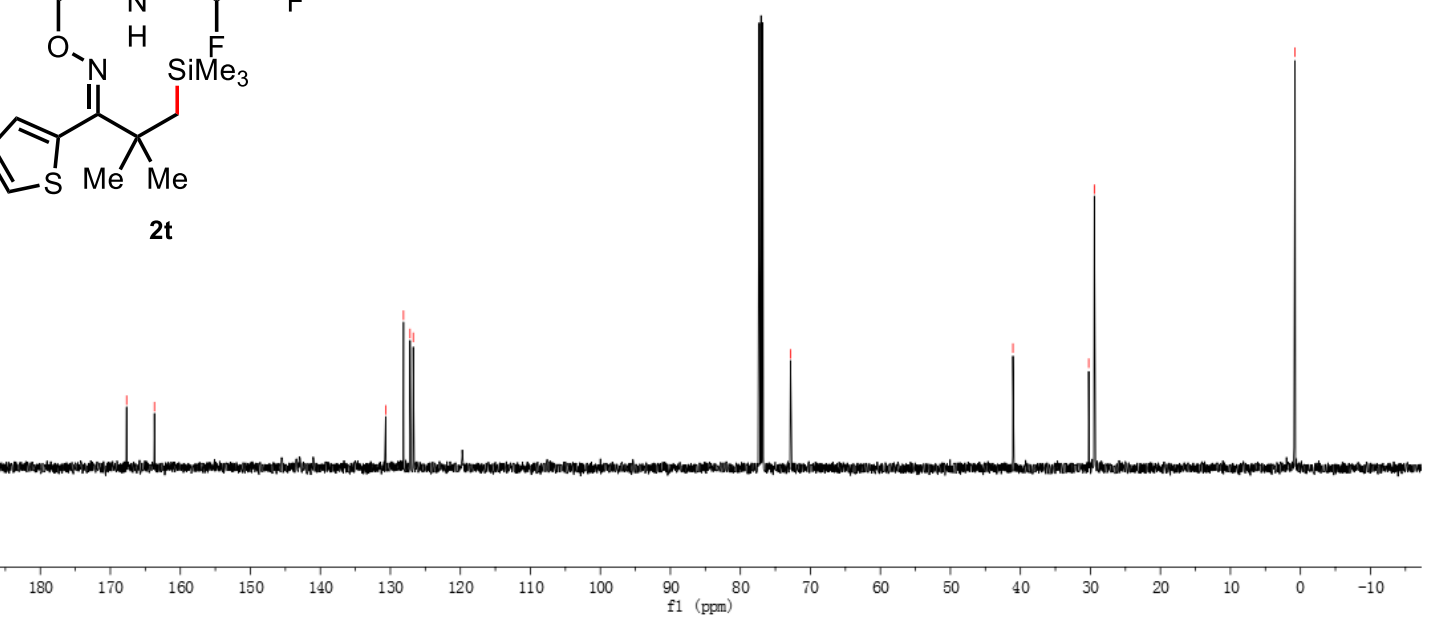
${ }^{1} \mathrm{H}$ NMR (500 MHz, $\left.\mathrm{CDCl}_{3}\right)$ spectra for $2 \mathrm{u}$

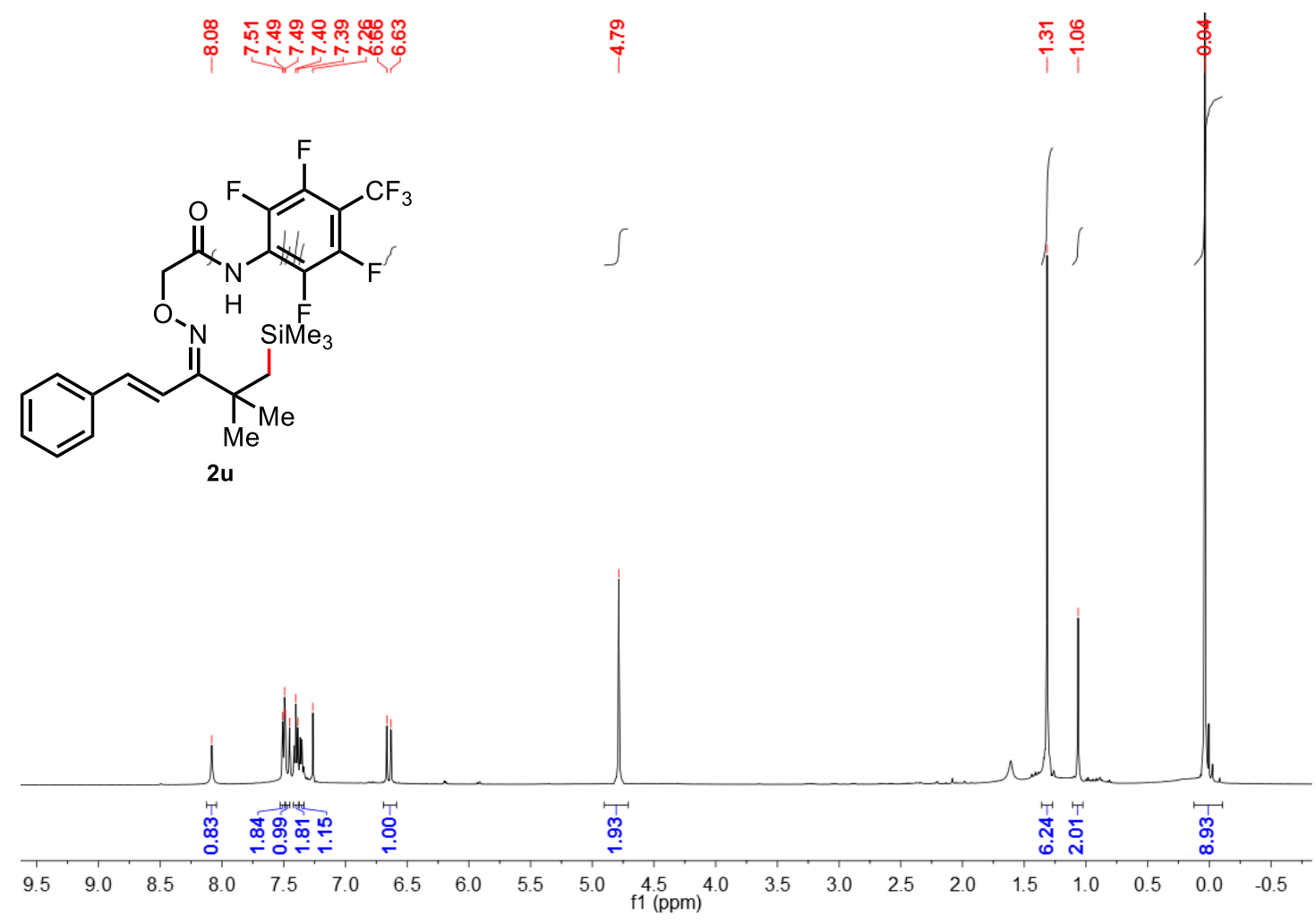

${ }^{13} \mathrm{C}$ NMR (126 MHz, $\left.\mathrm{CDCl}_{3}\right)$ spectra for $2 \mathrm{u}$

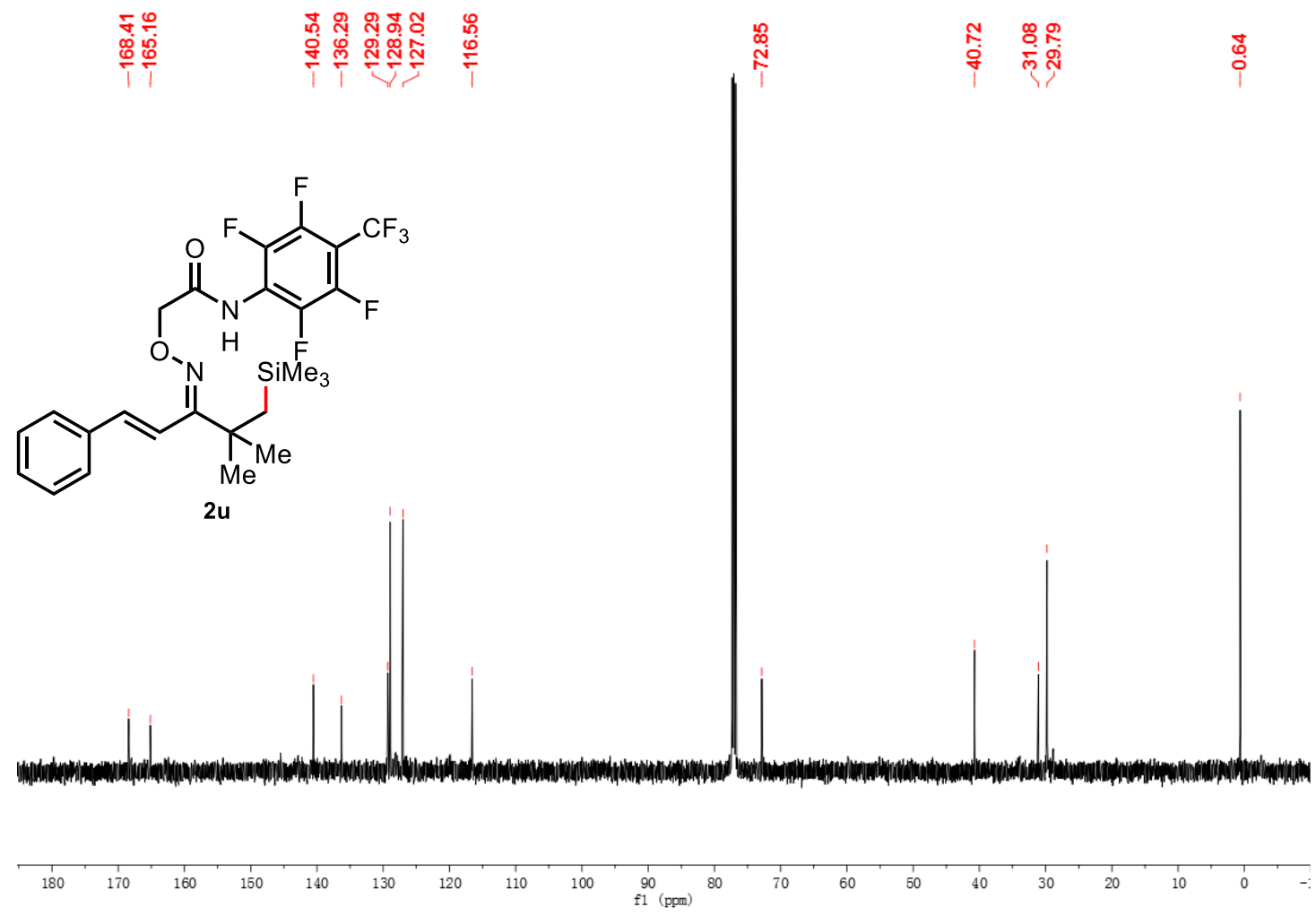


${ }^{1} \mathrm{H}$ NMR (500 MHz, CDCl 3$)$ spectra for $2 \mathrm{v}$

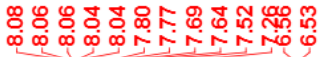

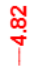

ํํำ

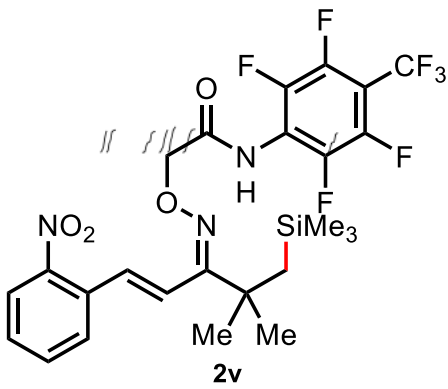
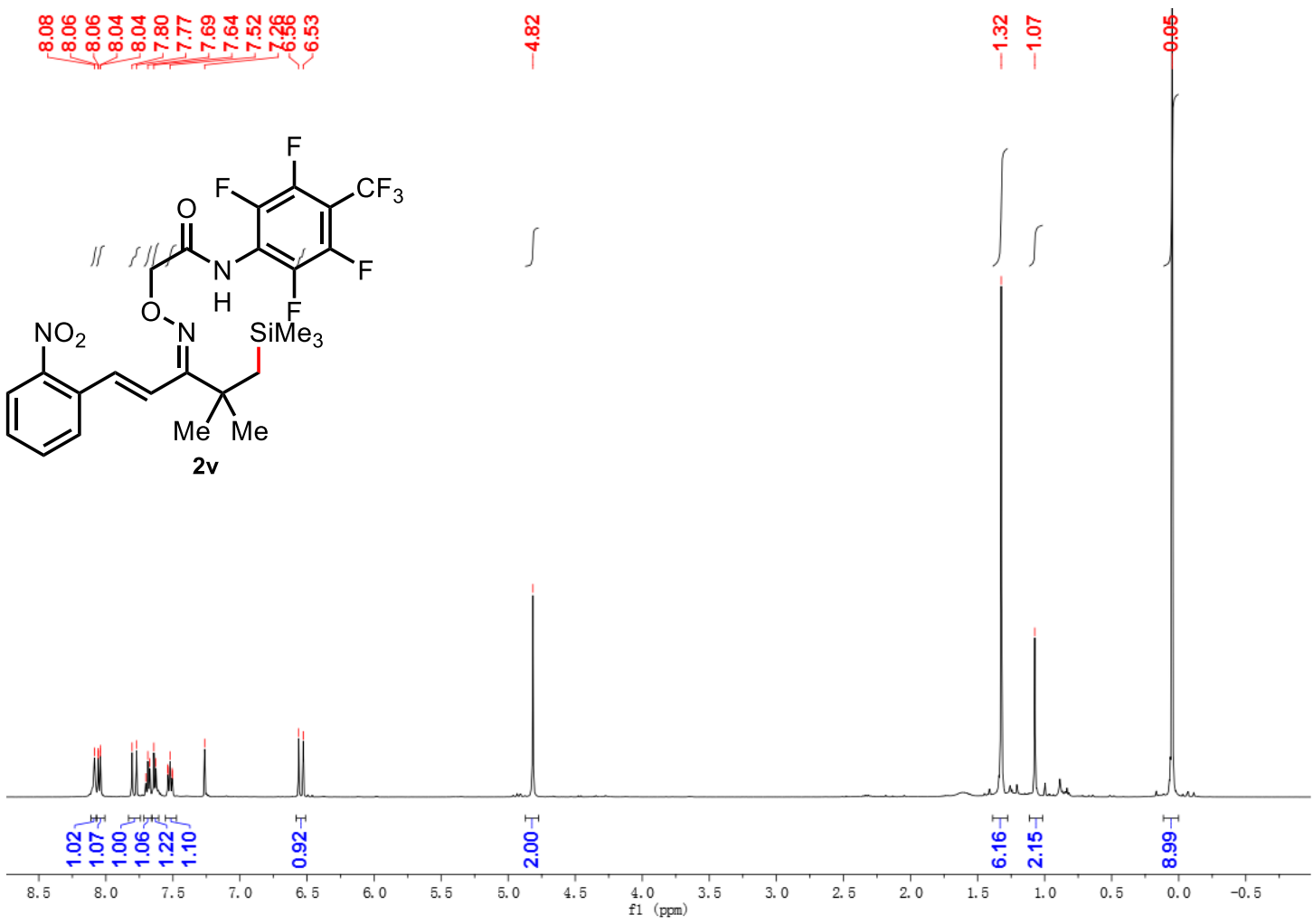

${ }^{13} \mathrm{C}$ NMR (126 MHz, $\mathrm{CDCl}_{3}$ ) spectra for 2v

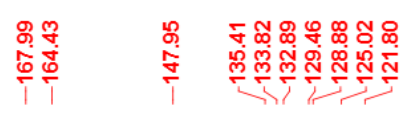

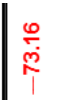

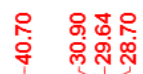

$\stackrel{8}{\circ}$<smiles>CCC(C)(C)C(/C=C/c1ccccc1[N+](=O)[O-])=N\OCC(=O)Nc1c(F)c(F)c(C(F)(F)F)c(F)c1F</smiles>

2v

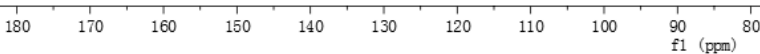


${ }^{1} \mathrm{H}$ NMR (500 MHz, $\left.\mathrm{CDCl}_{3}\right)$ spectra for $2 \mathrm{w}$

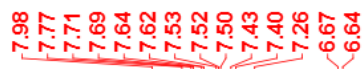
$\stackrel{\substack{i \\+}}{+}$<smiles>CS(C)(C)c1c(F)c(C(F)(F)F)c(F)c(F)c1NC(=O)CON</smiles>

NC<smiles>Cc1cccc(/C=C/C(=N)C(C)(C)C[Si])c1</smiles>

2w

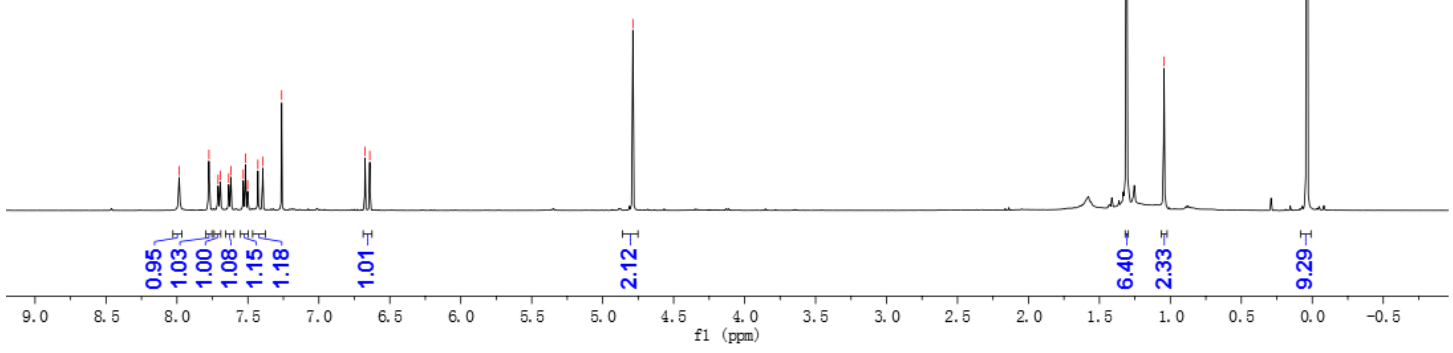

${ }^{13} \mathrm{C}$ NMR (126 MHz, $\left.\mathrm{CDCl}_{3}\right)$ spectra for $2 \mathrm{w}$
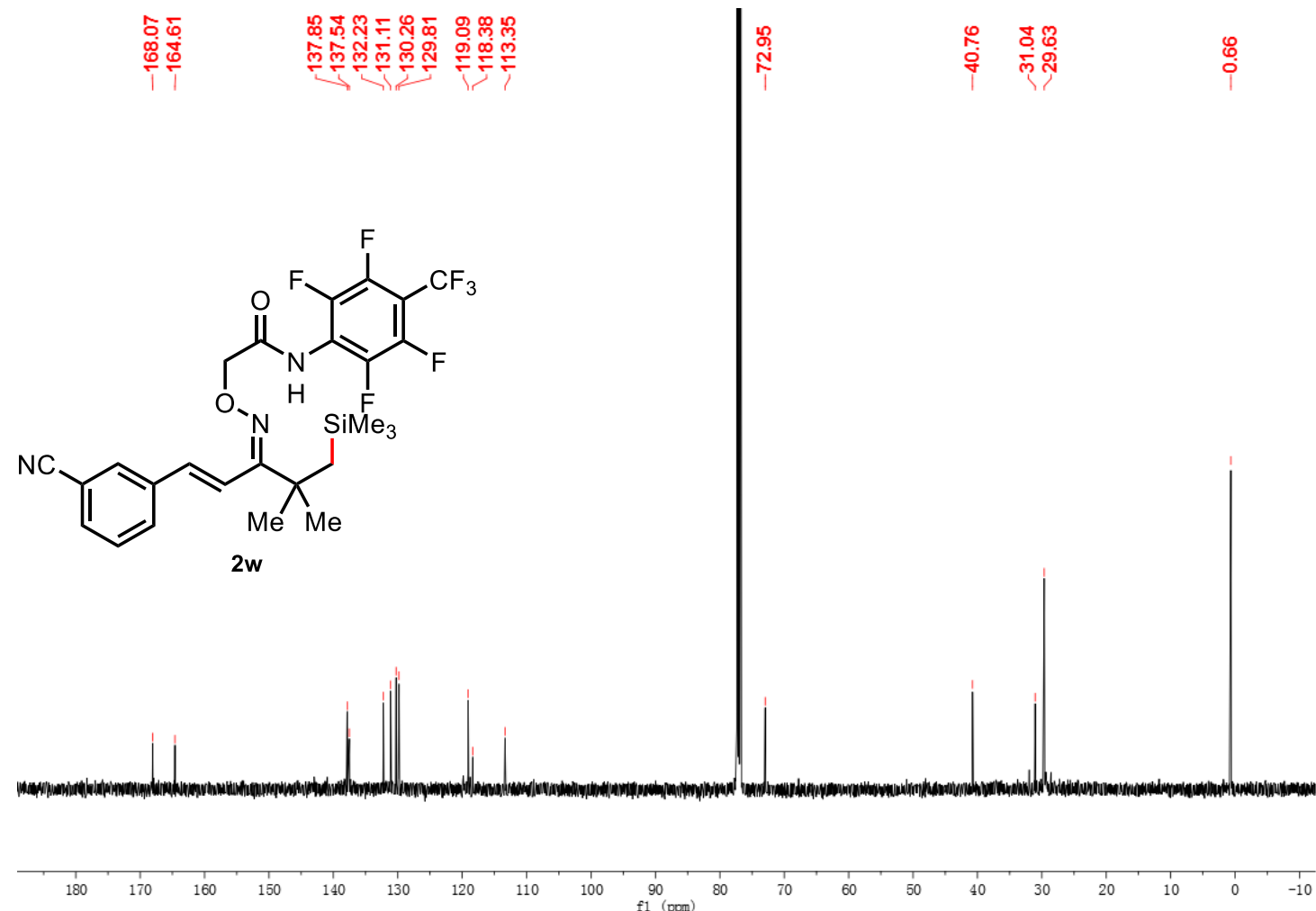
${ }^{1} \mathrm{H}$ NMR (500 MHz, $\mathrm{CDCl}_{3}$ ) spectra for $2 \mathrm{x}$

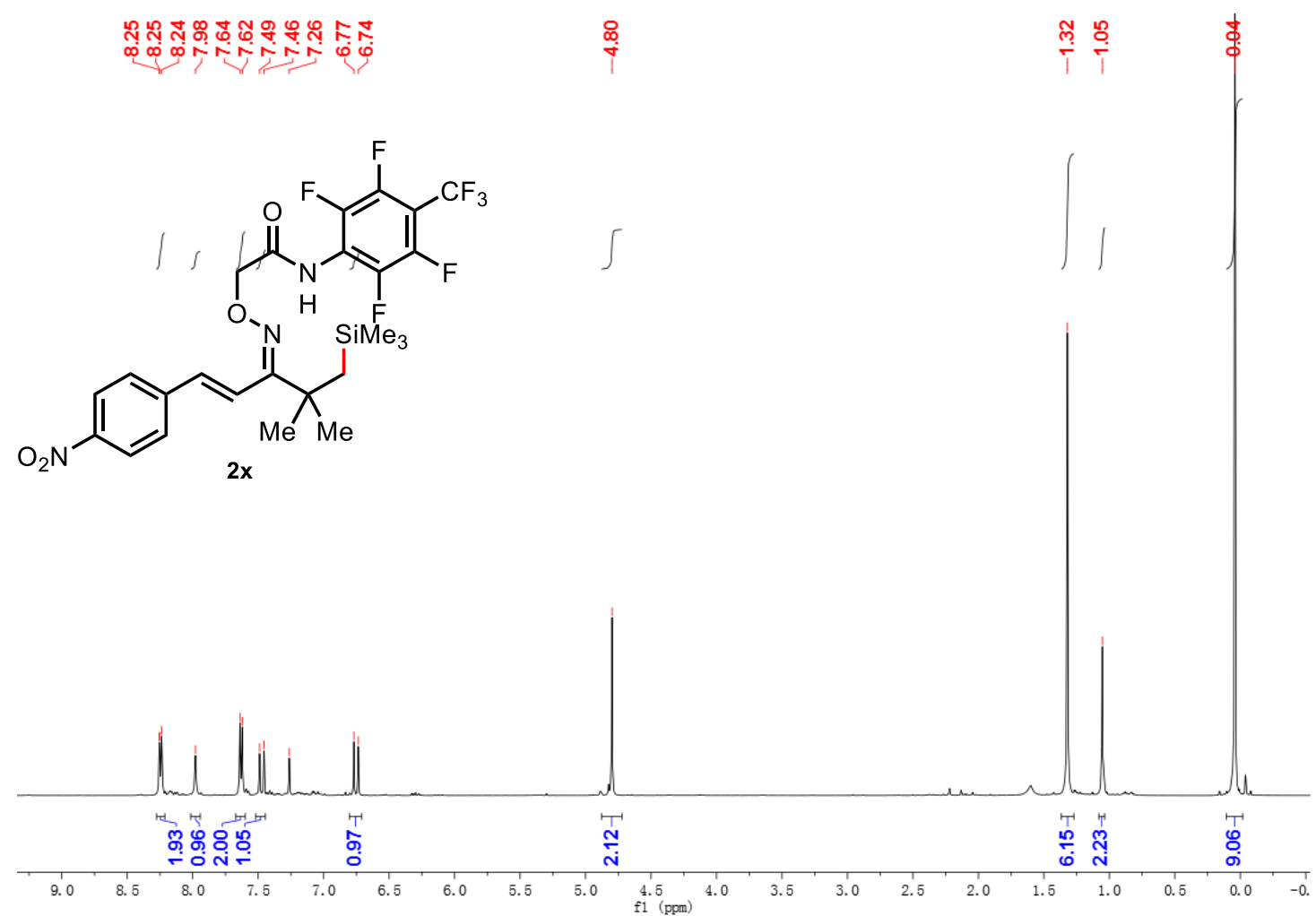

${ }^{13} \mathrm{C}$ NMR (126 MHz, $\mathrm{CDCl}_{3}$ ) spectra for $2 \mathrm{x}$

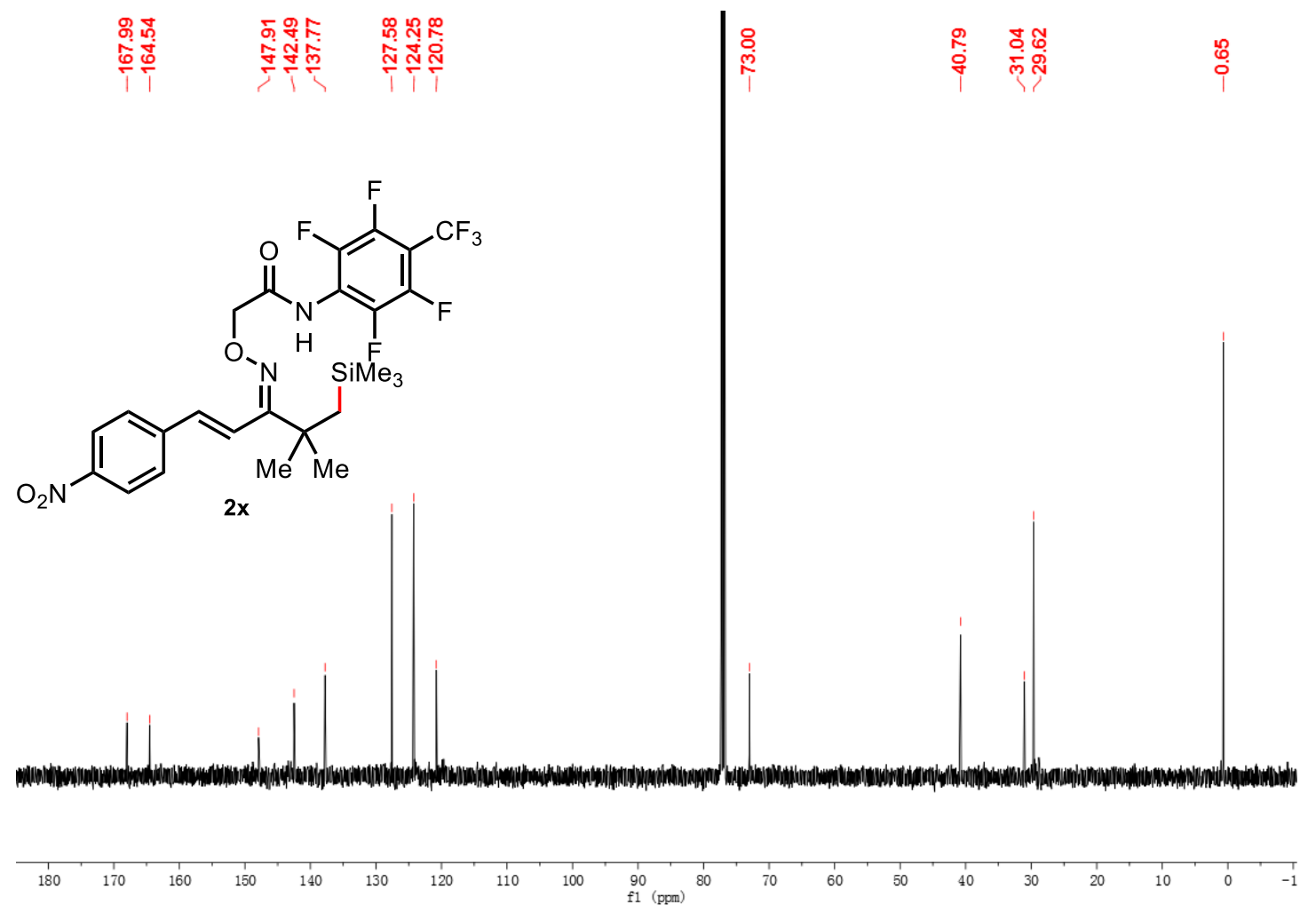


${ }^{1} \mathrm{H}$ NMR (500 MHz, $\mathrm{CDCl}_{3}$ ) spectra for $2 \mathrm{y}$

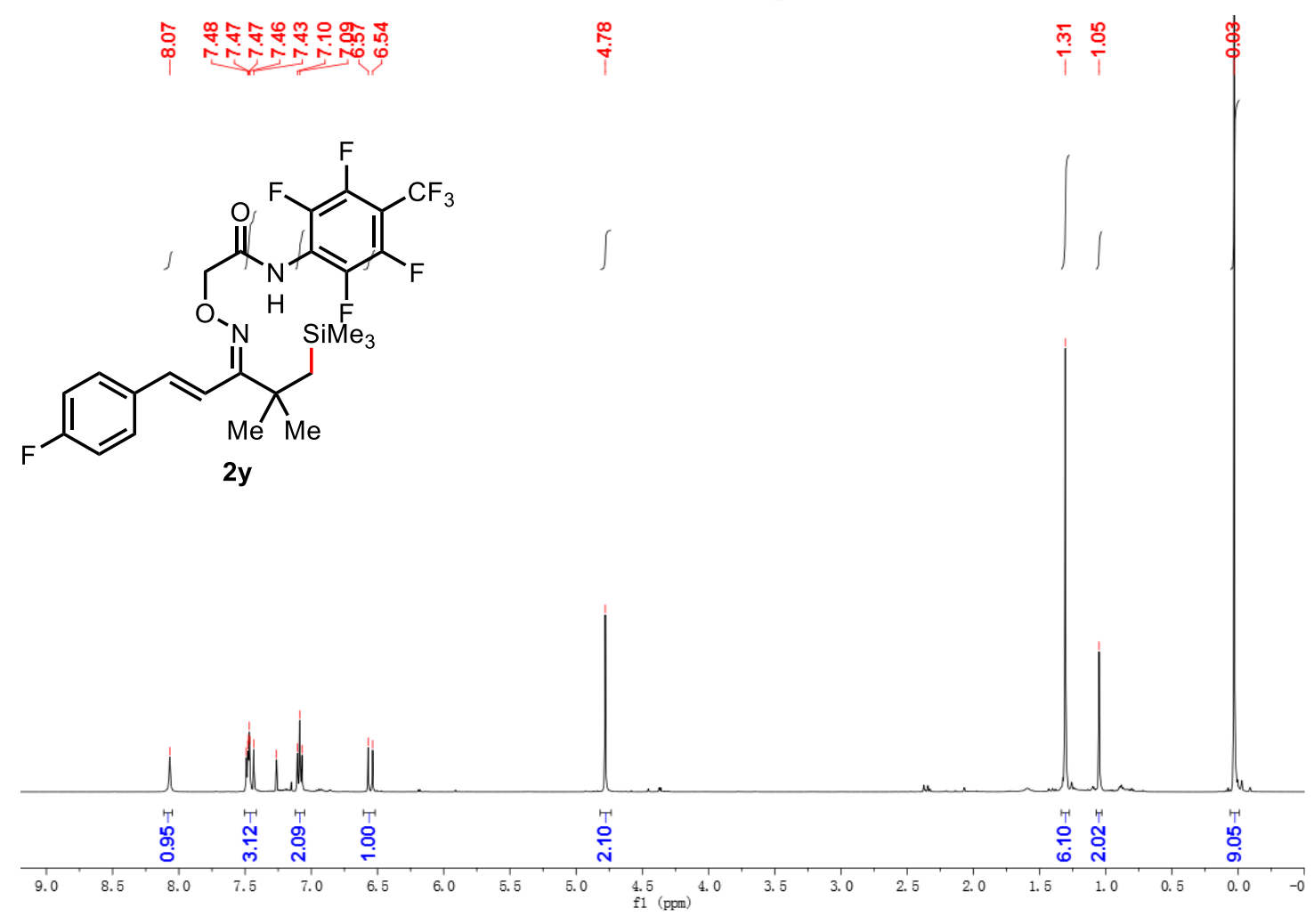

${ }^{13} \mathrm{C}$ NMR (126 MHz, $\mathrm{CDCl}_{3}$ ) spectra for $2 \mathrm{y}$
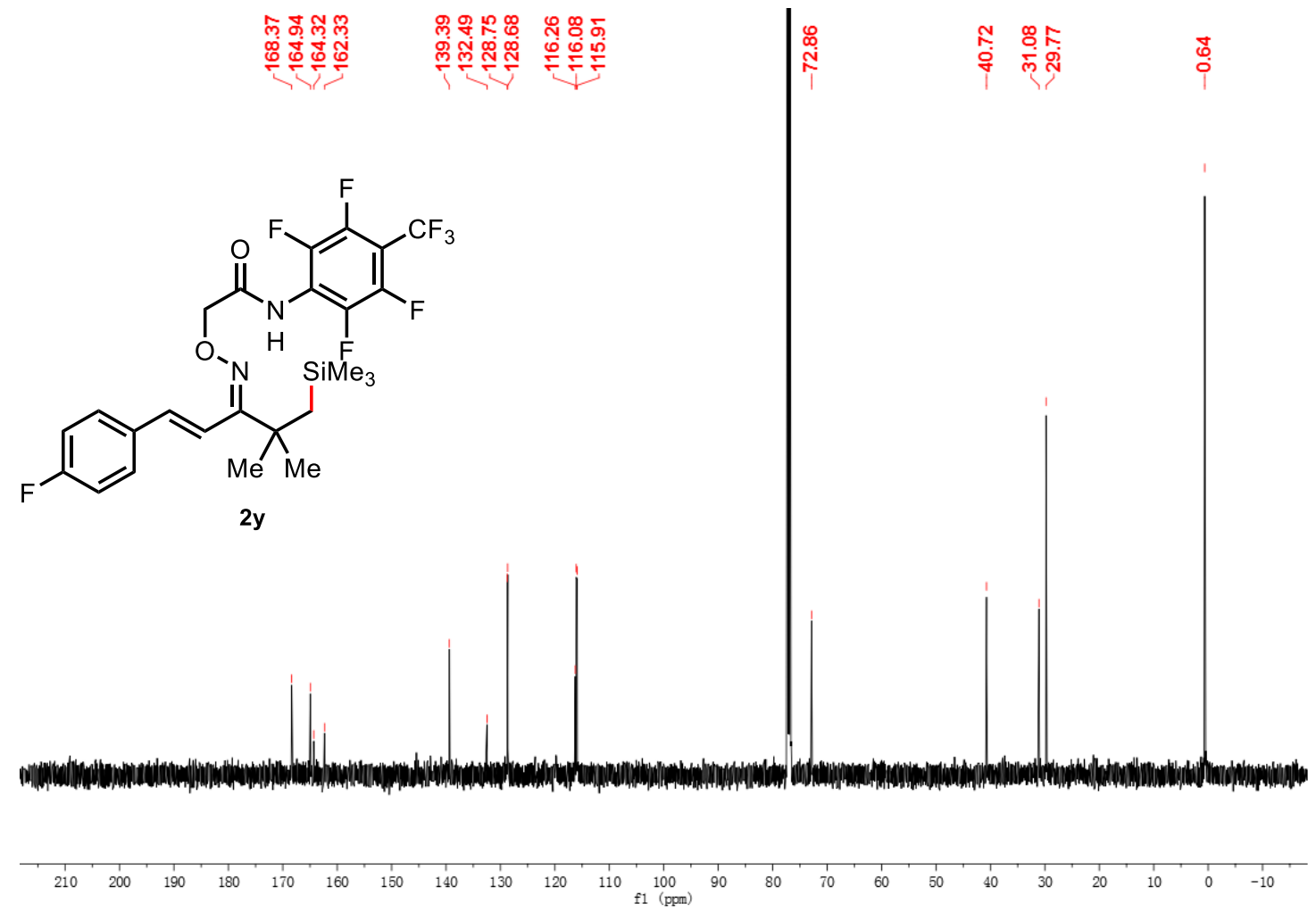
${ }^{1} \mathrm{H}$ NMR (500 MHz, $\mathrm{CDCl}_{3}$ ) spectra for $2 \mathrm{z}$

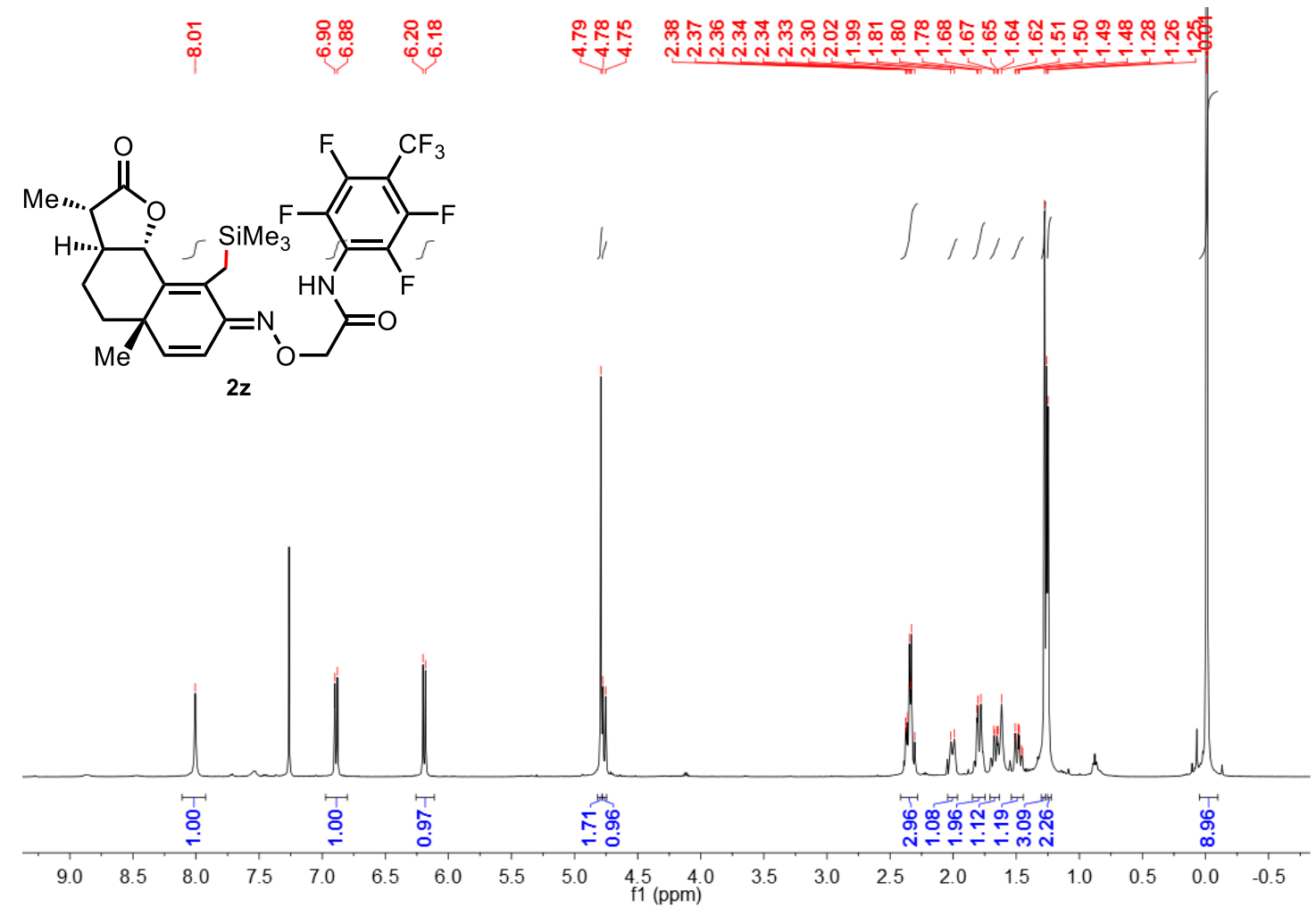

${ }^{13} \mathrm{C}$ NMR (126 MHz, $\left.\mathrm{CDCl}_{3}\right)$ spectra for $2 \mathrm{z}$

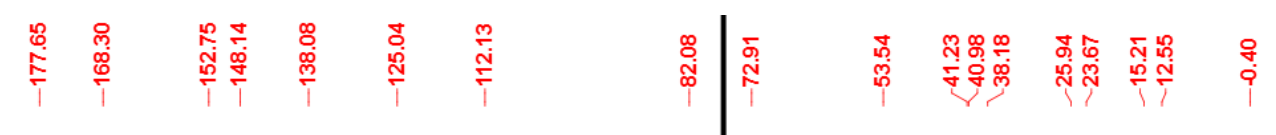<smiles>CCC1=C2[C@H]3OC(=O)[C@H](C)[C@H]3CC[C@]2(C)C=CC1=NOCC(=O)Nc1c(F)c(F)c(C(F)(F)F)c(F)c1F</smiles>

$2 z$

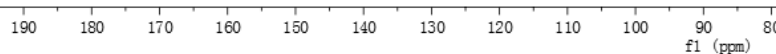


${ }^{1} \mathrm{H}$ NMR (500 MHz, $\mathrm{CDCl}_{3}$ ) spectra for 3d

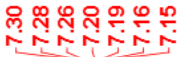

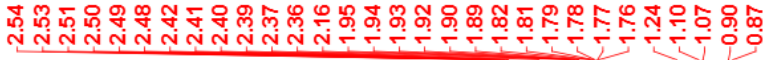

3d
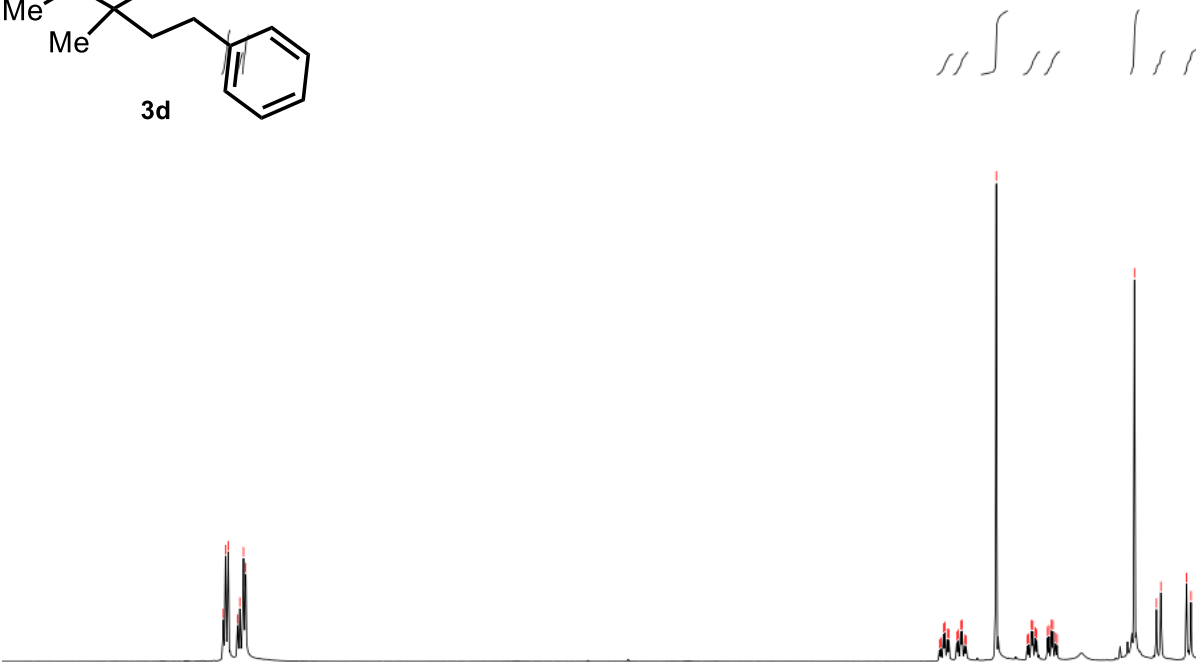

$\mid$ Wh wh
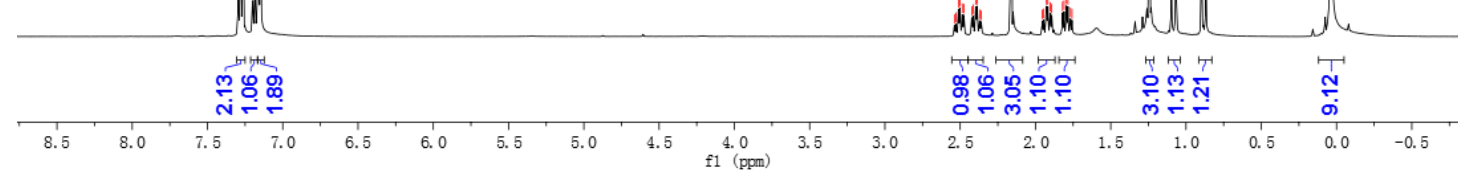

${ }^{13} \mathrm{C}$ NMR (126 MHz, $\mathrm{CDCl}_{3}$ ) spectra for 3d<smiles>CCC(C)(CCc1ccccc1)C(C)=O</smiles>

3d

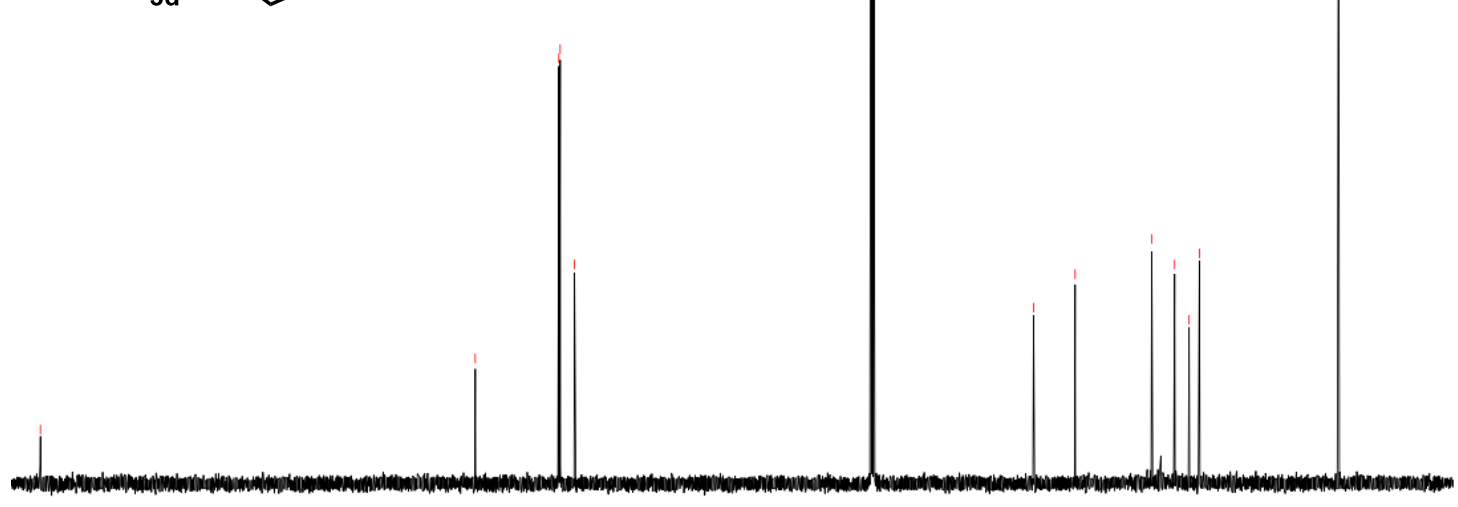

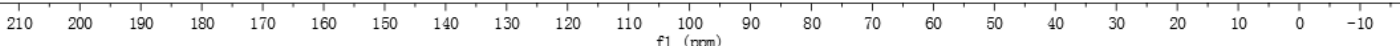

